\title{
The application of a \\ Pulsed Compression Reactor for the generation of syngas from methane
}

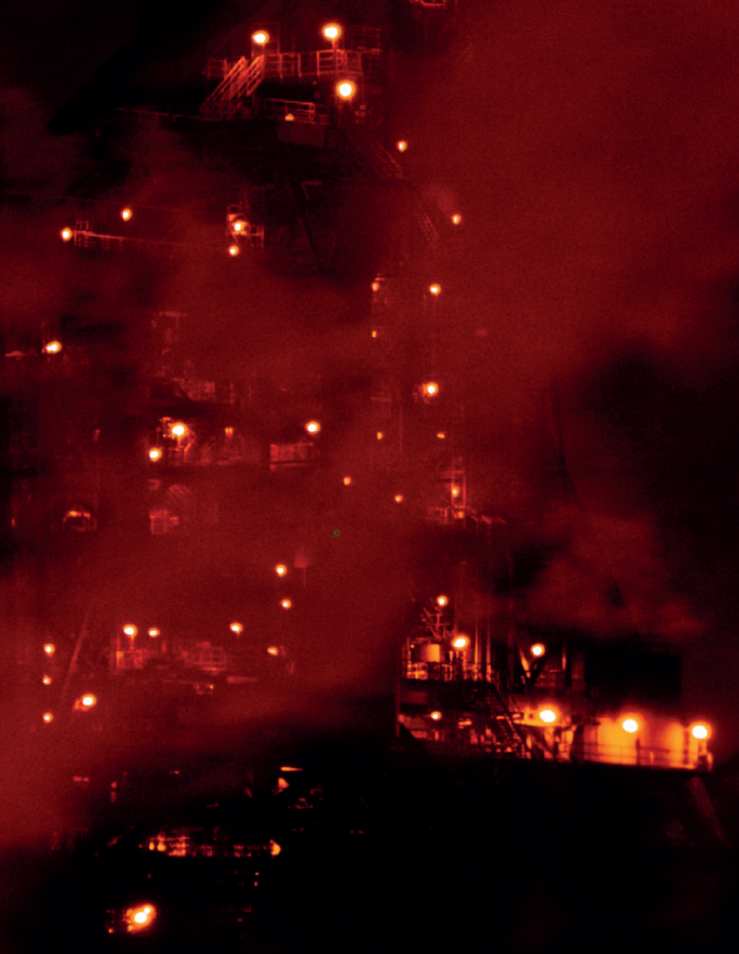

\section{Timo Roestenberg}
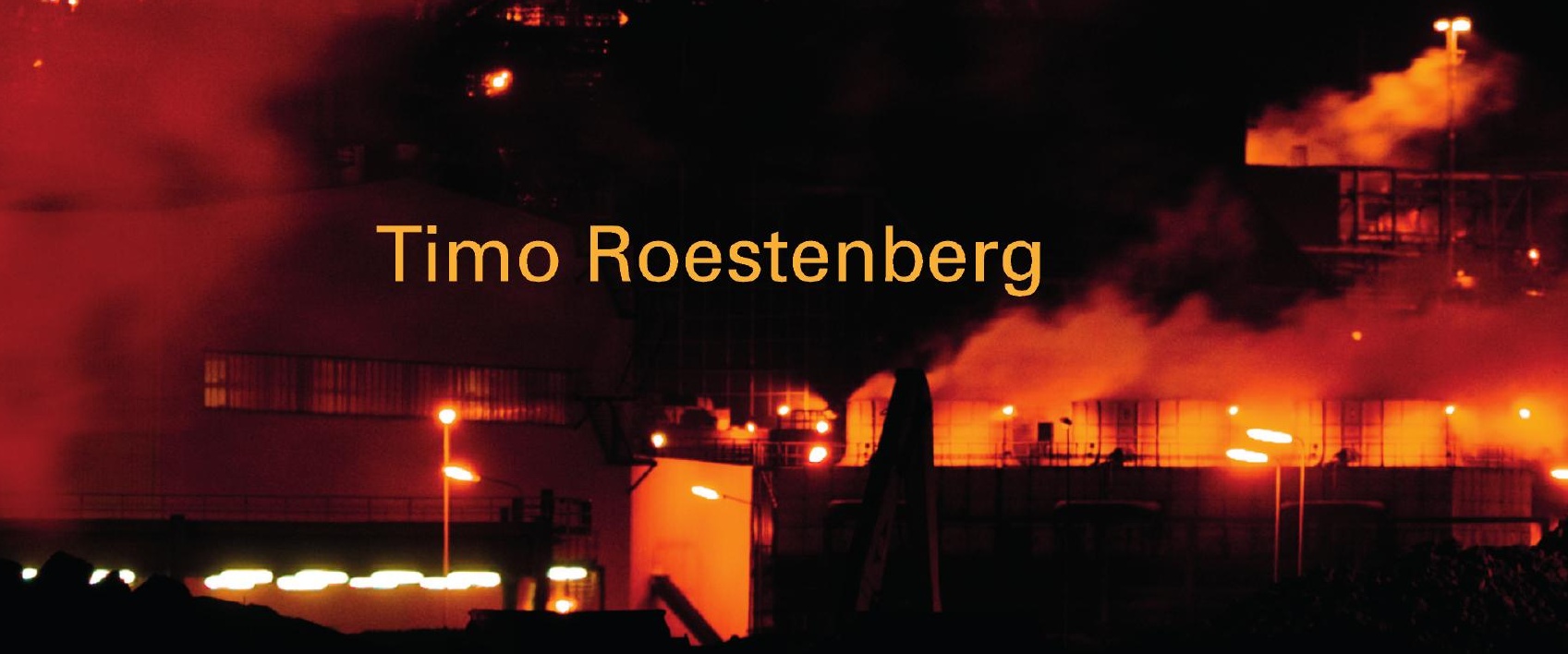


\section{The application of a pulsed compression reactor for the generation of syngas from methane}

Timo Roestenberg

21-01-2011 
Structure of the promotion committee:

chairman and secretary:

Prof. dr F. Eising

Universiteit Twente

promotor:

Prof. dr. ir. Th.H. van der Meer

Universiteit Twente

members:

Prof. dr. D. Lohse

Universiteit Twente

Prof. dr. ir. W.P.M. van Swaaij

Universiteit Twente

Prof. dr. ir. M. van Sint Annaland

Technische Universiteit Eindhoven

Prof. dr. ir. H.B. Levinsky

Rijksuniversiteit Groningen

referents:

Dr. H.P.C.E. Kuipers

Shell Global Solutions International BV

Dr. dr. ir. A.E. Kronberg

Energy Conversion Technologies BV

ISBN 978-90-365-3142-9

Keywords: Chemical reactors; Energy; Process control; Pulsed Compression Reactor; Reaction Engineering; Simulation

Cover photographs courtesy of BPW Roestenberg photography, www.roestenberg.nl

The work in this thesis was performed as part of SenterNovem project EOS-LT 04025

Copyright (C) 2010 by T. Roestenberg, Enschede, The Netherlands

All rights reserved. No parts of this publication may be reproduced, stored in a retrieval system, or transmitted in any form or by any means, electronic, mechanical, photocopying, recording or otherwise, without the prior written permission of the copyright holder. 


\title{
THE APPLICATION OF A PULSED COMPRESSION REACTOR FOR THE GENERATION OF SYNGAS FROM METHANE
}

\author{
DISSERTATION
}

to obtain

the degree of doctor at the University of Twente, on the authority of the rector magnificus, prof. dr. H. Brinksma, on account of the decision of the graduation committee, to be publicly defended on Friday the $21^{\text {st }}$ of January 2011 at 16:45

by

Timo Roestenberg

born on April 21, 1983

in De Bilt 
This dissertation has been approved by the promoter:

Prof. dr. ir. Th.H. van der Meer 


\section{Summary}

The idea of using compressive heating for a range of chemical reactions, other than combustion reactions in an internal combustion engine, has been around for almost a century, but has never been applied on a large industrial scale. This may be considered odd, since heating reactants by compression is much more energy efficient than heating by any other means. This gain in energy efficiency lies in the effectiveness with which the energy can be recuperated. While heat that is supplied for instance by the combustion of fossil fuels may be recuperated by using expensive high temperature heat exchangers (invariably with large energy losses), the heat that is added by compression of the feed stream can be much more easily recuperated by allowing the products to perform work while expanding, and using the work performed for the compression of the next batch of reactants. In this way energy losses and degradation can be reduced drastically.

Recent work of Glouchenkov [15] has resulted in a reactor concept that utilizes the concept of (near) adiabatic compression and is believed to be viable on industrial scale. This pulsed compression reactor (PCR) promises to be a highly energy efficient alternative for conventional chemical reactors, for high temperature processes. However, since the technology is still in its infancy stage, much research needs to be done in order to make the technology industrially ripe.

The research presented in this thesis is aimed at gaining some fundamental insight in the operation of the PCR in general, as well as the specific application for syngas generation from methane. This research can be divided into three parts: an investigation of heat transfer from the hot gas to the reactor walls and piston, an investigation of the chemistry of both partial oxidation of methane as well as steam reforming and the investigation of the stability of the PCR piston reciprocation.

To investigate the heat transfer from the hot gas to the reactor walls and piston two approaches are used. Firstly, the continuous average heat transfer is measured in a continuously reciprocating reactor. This is done for various operating conditions, at relatively low pressures. The data obtained is used to derive an empirical heat transfer coefficient relation, valid for the operational domain under investigation. With these experiments an experimental method is developed that can be applied in a larger range of operating conditions. The second approach uses a single shot reactor that mimics a single compression expansion stroke of the PCR. During this one stroke, the instantaneous heat flux from the gas to the reactor cover is measured using a so-called 
eroding thermocouple. This is used to derive an empirical relation between the heat loss from the compressed gas in this single shot reactor and the compression pressure. This relation gives insight into the effect that the reactor walls and piston have on the chemistry occurring in the single shot reactor.

In the investigation of syngas generation from methane, the chemistry of both partial oxidation and steam reforming of methane are investigated in the single shot reactor. This is done both experimentally and by simulations of the process using models with detailed chemistry. Several parameters are isolated: the maximum piston kinetic energy, initial reactor and reactant temperature, initial reactant mixture composition, cover geometry and piston mass. From the data obtained both from experiments and simulations it is concluded that partial oxidation of methane in the PCR is a viable application of the PCR: hydrogen and carbon monoxide yields were above $85 \%$, with soot formation below $5 \%$ (depending on the mixture used and the initial temperature). Models showed a good correspondence with the experimental data. The process of pure steam reforming, where only methane and steam were the reactants, yielded lesser results in terms of carbon monoxide formation, due to higher soot formation, but many options for improving the results are still open for investigation. It was also shown that methane pyrolysis with ethane, ethylene, acetylene and hydrogen as the intended products is feasible in the PCR. The simulation models used deviated much more from the experiments in the case of steam reforming, which can be attributed to the absence of a soot formation model in the simulation mechanisms.

Lastly, an analysis of the experimental and numerical data obtained yielded a theory that describes the behavior of the PCR in continuous reciprocation with respect to reciprocation stability. It was shown that, if a point exists where the energy release of chemical reactions exactly compensates the energy losses, reciprocation will always converge to this point or cease. This is an important result with respect to the safety issues associated with the PCR operation.

The fundamental investigations presented in this thesis show that the PCR is a good alternative to conventional chemical reactors, especially where high temperature processes are involved. The PCR promises to be an energy efficient option. 


\section{Samenvatting}

Het idee om verwarming van gassen door middel van compressie te gebruiken in een scala van chemische reacties, buiten verbrandingsreacties in interne verbrandingsmotoren, bestaat al meer dan een eeuw, maar is nooit op industriële schaal toegepast. Dat kan als vreemd worden beschouwd, aangezien het verwarmen van gassen door samendrukken een veel energie-efficiëntere methode kan zijn dan welke methode dan ook. Deze verbetering in energie-efficiëntie ligt in de effectiviteit waarmee de energie terug gewonnen kan worden. De warmte die bijvoorbeeld is toegevoegd door de verbranding van fossiele brandstoffen alleen terug gewonnen kan worden door gebruik te maken van dure warmtewisselaars bestendig tegen hoge temperaturen (altijd gepaard met grote energie verliezen). De warmte welke door samendrukking van de reactanten stroom toegevoegd kan simpelweg terug gewonnen worden door de producten arbeid te laten verrichten tijdens de expansie, en deze arbeid te gebruiken voor het comprimeren van de volgende portie reactanten. Op deze manier kunnen energie verliezen en degradatie drastisch verminderd worden.

Recent werk van Glouchenkov [15] heeft geresulteerd in een reactorconcept dat gebruik maakt van (bijna) adiabatische compressie en levensvatbaar lijkt te zijn op industriële schaal. Deze gepulseerde compressie reactor (Pulsed Compression Reactor, PCR) belooft een zeer energie-efficiënt alternatief te zijn voor conventionele reactoren, voor hoge temperatuur processen. Echter, aangezien deze technologie redelijk nieuw is, moet nog veel onderzoek verricht worden om de technologie industrierijp te maken.

Het onderzoek dat in dit proefschrift gepresenteerd wordt is gericht op het verkrijgen van fundamenteel inzicht in de PCR in het algemeen, alsmede de specifieke applicatie van het maken van synthesegas uit methaan. Het onderzoek kan onderverdeeld worden in drie delen: een onderzoek naar de warmte overdracht tussen het hete gas en de reactor wanden en zuiger, een onderzoek naar de chemie van zowel de partiële oxidatie van methaan als ook het reformen met stoom en een onderzoek naar de stabiliteit van de PCR zuigerbeweging.

Om de warmteoverdracht tussen de hete gassen en de reactor-wanden en zuiger te onderzoeken worden twee benaderingen gebruikt. Ten eerste wordt de continue gemiddelde warmteoverdracht gemeten in een continu geopereerde reactor. Dit wordt gedaan voor verschillende bedrijfscondities, bij relatief lage drukken. De verkregen data wordt gebruikt om een empirische relatie te verkrijgen voor de 
warmteoverdrachtscoëfficiënt, welke geldig is voor het onderzochte bedrijfsgebied. De tweede benadering maakt gebruik van een één schots reactor die een enkele compressie expansie slag van de PCR nabootst. Tijdens deze ene slag wordt de warmteflux van het gas in de reactor gemeten met een zogenaamd eroderend thermokoppel. Dit wordt gebruikt om een empirische relatie tussen de warmteverliezen van het gas in deze één schots reactor en de druk te vinden. Deze relatie geeft inzicht in het effect dat de koude reactorwanden en zuiger hebben op de chemie in de één schots reactor.

In het onderzoek naar de syngas vorming uit methaan, wordt de chemie van zowel partiële oxidatie en stoom reformen onderzocht in de één schots reactor. Dit wordt gedaan door middel van experimenten en door simulaties van het proces met modellen met gedetailleerde chemie. Verschillende parameters worden geïsoleerd: de maximale kinetische energie van de zuiger, initiële temperatuur van de reactor en reactanten, initiële reactanten compositie, dekselgeometrie en zuigermassa. Uit de data welke verkregen is uit zowel experimenten en simulaties wordt geconcludeerd dat partiële oxidatie van methaan in de PCR een levensvatbare toepassing van de PCR is: waterstof en koolmonoxide opbrengsten waren boven de $85 \%$ met roetvorming onder de $5 \%$ (afhankelijk van het mengsel dat gebruikt wordt en de initiële temperatuur). Modellen laten een goede overeenstemming met de experimentele data zien. Het proces van puur stoom reformen, waar methaan en stoom de enige reactanten waren, gaf mindere resultaten in termen van koolmonoxide vorming, door hogere roetvorming, maar er zijn nog veel mogelijkheden voor verbetering van de resultaten open voor onderzoek. Het was ook aangetoond dat pyrolyse van methaan, met als beoogde producten ethaan, ethyleen, acetyleen en waterstof haalbaar zijn in de PCR. De simulatiemodellen weken meer af van de experimentele data in het geval van stoom reformen, door het ontbreken van een roetvormingsmodel in de simulatiemechanismen.

Tot slot heeft de analyse van de vergaarde experimentele en numerieke data geleid tot een theorie welke het gedrag van de continue bewegende PCR met betrekking tot de stabiliteit beschrijft. Het is aangetoond dat, indien er een punt bestaat waar de warmteontwikkeling van de chemische reacties de warmteverliezen exact compenseren, de op en neer beweging van de zuiger altijd naar dit punt zal convergeren of zal stoppen. Dit is een belangrijk resultaat met betrekking tot de veiligheid van de PCR werking.

Het fundamentele onderzoek dat in dit proefschrift gepresenteerd wordt laat zien dat de PCR een goed alternatief is voor conventionele reactoren, vooral wanneer hoge temperatuur processen een rol spelen. De PCR beloofd een energie-efficiënte optie te zijn. 


\section{Contents}

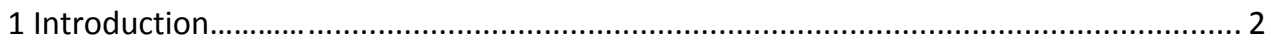

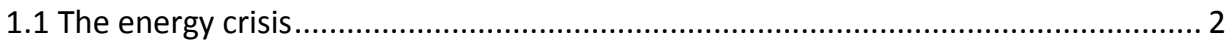

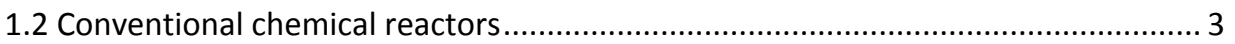

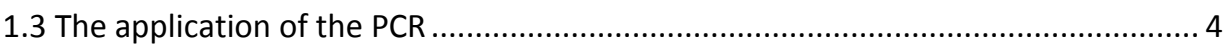

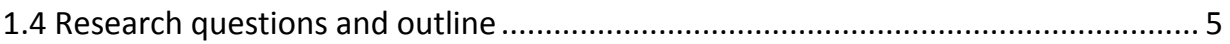

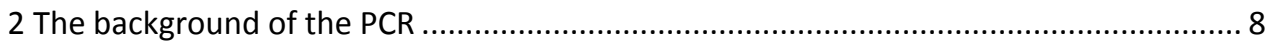

2.1 The internal combustion engine as a chemical reactor ......................................... 8

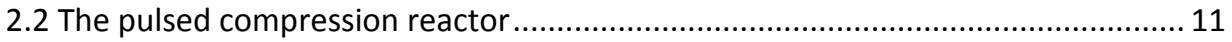

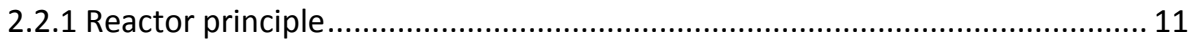

2.2.2 The principle of compressive heating ......................................................... 15

2.2.3 Different operation modes ...................................................................... 16

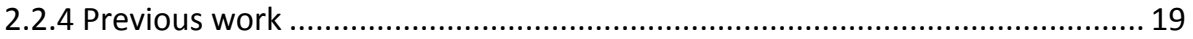

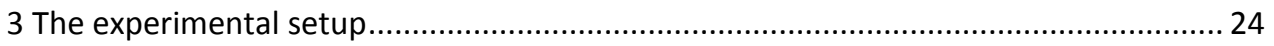

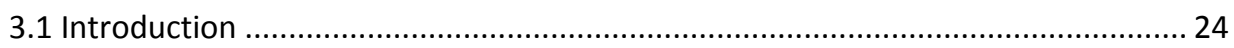

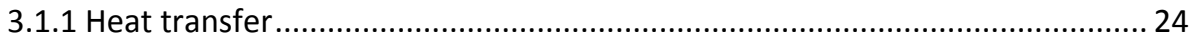

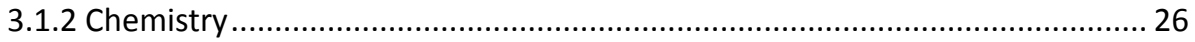

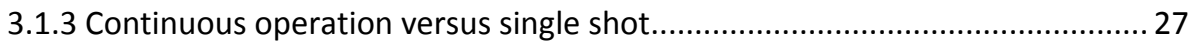

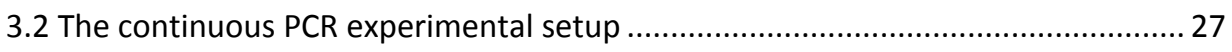

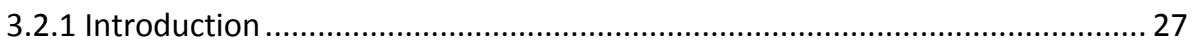

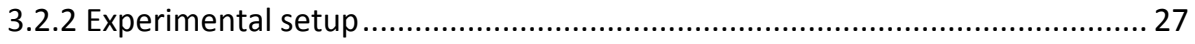

3.2.3 Experimental procedure for average heat flux measurement ....................... 32

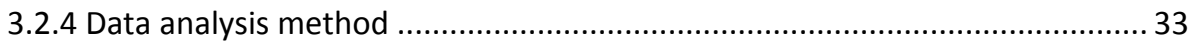

3.3 The single shot reactor experimental setup..................................................... 40

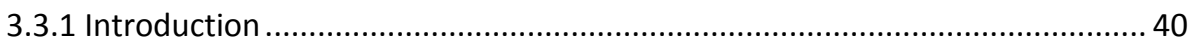

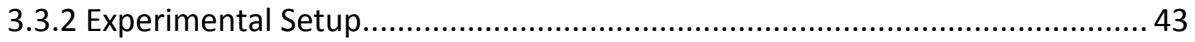

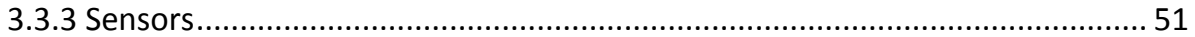

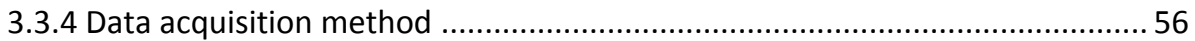


3.3.5 Instantaneous heat flux data analysis method ............................................. 57

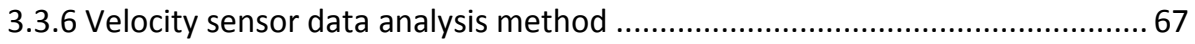

3.3.7 Chemistry experiments experimental method and data analysis .................. 70

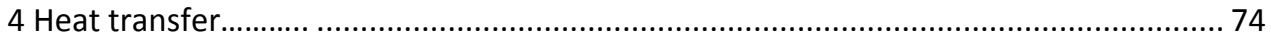

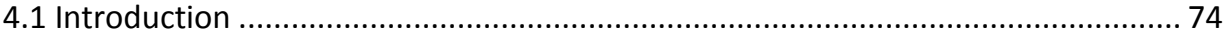

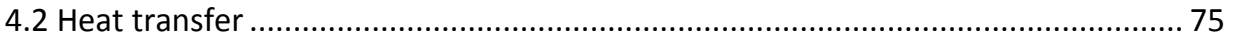

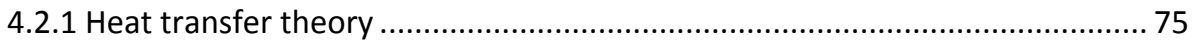

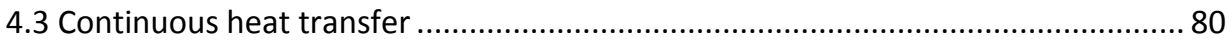

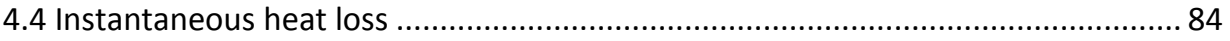

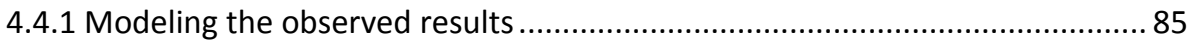

4.4.2 Comparing instantaneous heat losses to averaged heat transfer .................. 87

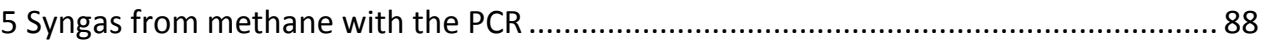

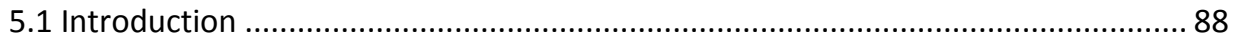

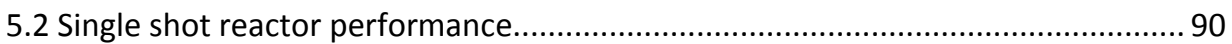

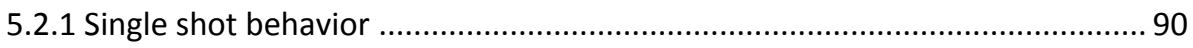

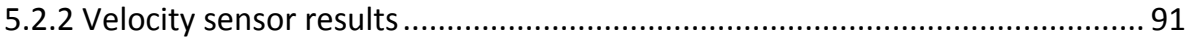

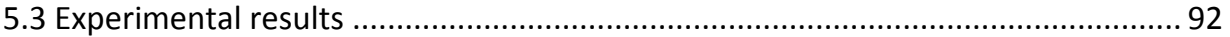

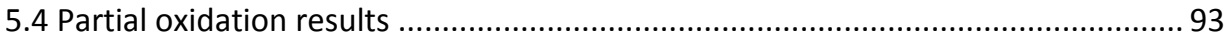

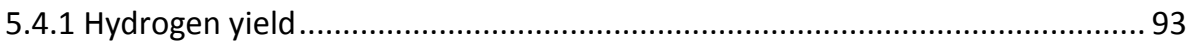

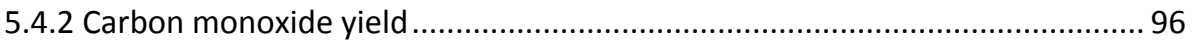

5.4.3 Oxygen concentration in product gas.......................................................... 99

5.4.4 Minor species by partial oxidation........................................................... 100

5.4.5 Hydrogen - carbon monoxide ratio.......................................................... 105

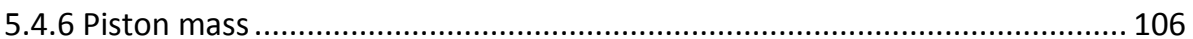

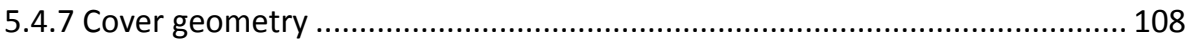

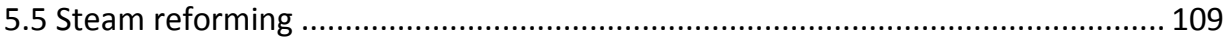

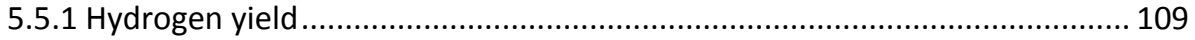

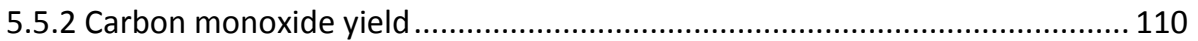

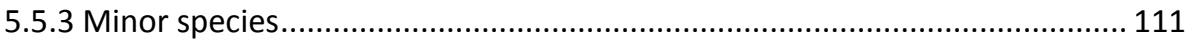




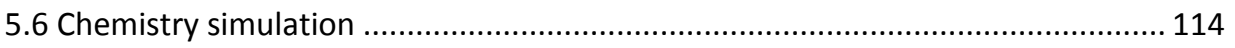

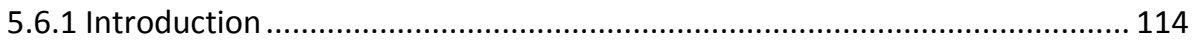

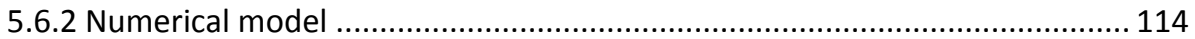

5.6.3 Reaction mechanisms ................................................................................ 115

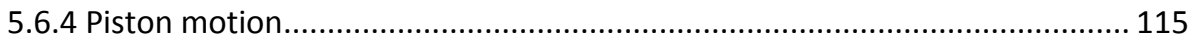

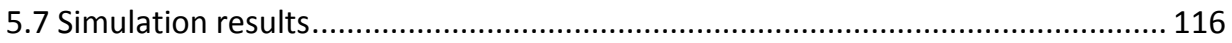

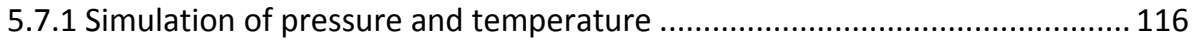

5.7.2 Partial oxidation yields compared to measurements ................................. 118

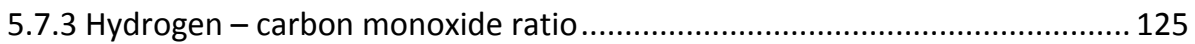

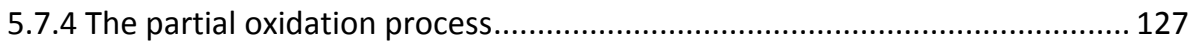

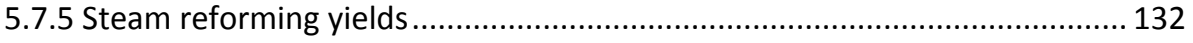

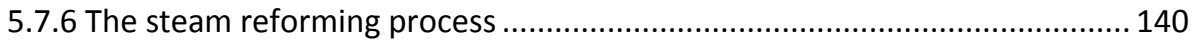

6 The controllability and run-away possibility of the PCR .......................................... 144

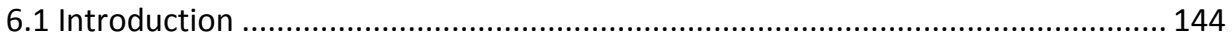

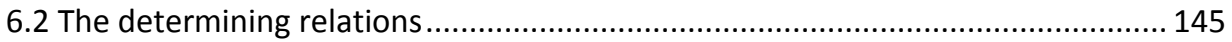

6.3 The relation between energy input and ignition moment.................................. 146

6.4 The relation between ignition moment and change of kinetic energy of the piston .. 149

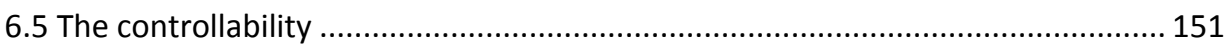

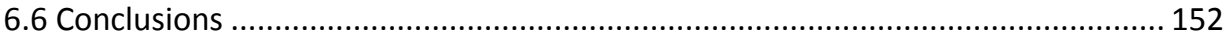

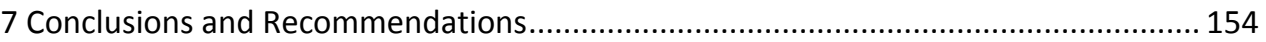

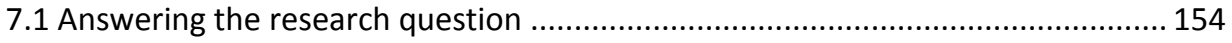

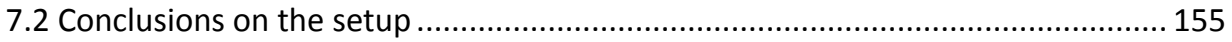

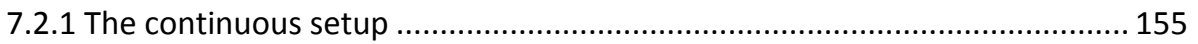

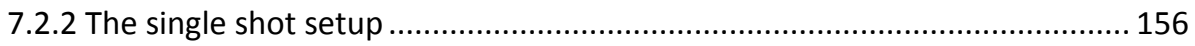

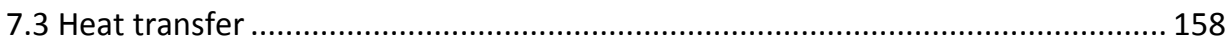

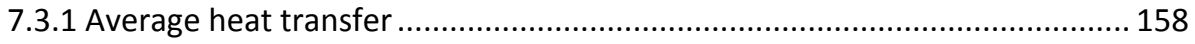

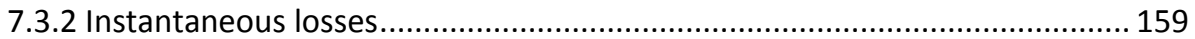

7.3.3 Average and instantaneous losses compared ............................................. 159

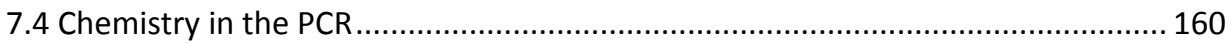


7.4.1 Experiments on syngas formation by partial oxidation in the PCR ................ 160

7.4.2 Experiments on syngas formation by steam reforming in the PCR ............... 162

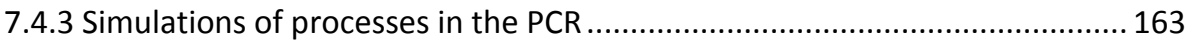

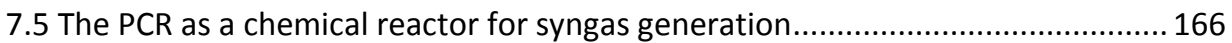

7.6 The controllability and run-away possibility of the PCR....................................... 166

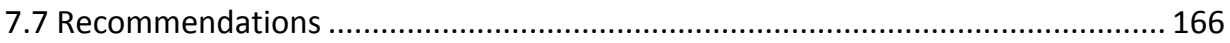

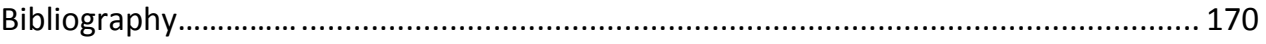

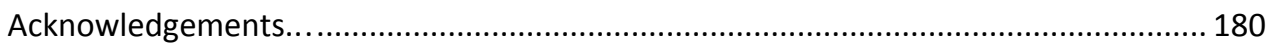

I Different high speed surface temperature sensors................................................ 182

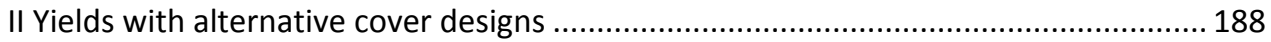




\section{Introduction}

The pulsed compression reactor (PCR) is a new technology that has been under investigation over the last few decades. While the fundamentals of the technology have been known for almost a century, the specific application that is investigated is still in its infancy stage. This means that a lot of developments are still to be made in order to make pulsed compression technology industry ripe.

In this first chapter an introduction is given on the research that is performed to advance the PCR towards a mature, industrially ripe reactor. This begins with the background information that prompted the development of the PCR and an explanation of the specific application of the PCR that is investigated, followed by the goals of the research.

\subsection{The energy crisis}

After many years of debate, there finally seems to be consensus among most people that climate change is in fact happening and that our emissions are to blame. The general public is more and more starting to realize that the adaptation of more energy efficient and sustainable processes is vital. This view is starting to be reflected in political agendas of governments and government agencies, as well as the mission statements of many private companies.

One of the mayor problems in shifting to sustainable energy sources is the human dependency on fossil fuels. Essentially the entire world infrastructure is based on the use of fossil fuels, meaning that the use of other energy sources implies a great infrastructural change. So, instead of using a totally different type of energy source, a more ecologically friendly replacement to fossil fuels may be much more attractive.

At the same time, great profit in terms of emission reduction can be made by developing more energy efficient processes and systems. Ideally efforts are divided among making existing processes and systems more energy efficient, creating sustainable alternatives to fossil fuels as well as developing new processes that do not require fossil fuels at all. 
The technology introduced and investigated in this project aims to be a much more energy efficient and compact alternative for conventional chemical reactors. It can be applied for both fossil as well as alternative fuels.

\subsection{Conventional chemical reactors}

There are many types of reactors, adapted for the thousands of chemical processes. Overviews of the different types can be found in, for example, [1] and [2].

While various reactors are very different from each other in some aspects (both in functionality as in application), their common problem is the recovery of heat. At the same time, demands on construction materials for high temperature reactors are very high. Especially if a combination of high temperature and pressure is required.

A great variety of chemical production processes require high reaction temperatures. Good examples of these are the production of synthesis gas based products such as synthetic fuels and ammonia, dehydrogenation processes, pyrolysis for the production of ethylene and acetylene and many others. As a result up to $70 \%$ of the capital and operating cost of the production of, for example, synthetic fuels, lies in the synthesis gas plant.

Not only does the requirement of high temperatures (and pressures) make these production processes more complicated, and invariably more expensive, it also leads to significant energy losses. Each compression step, heating step or in fact any other step that requires energy transformation from one form to another, inevitably introduces energy and exergy losses. Looking at the synthesis gas based products, from $30 \%$ (in the case of methanol from natural gas) to $50 \%$ (in the case of Fischer-Tropsch synthesis products from coal) of the raw material potential energy is lost [3].

Because of materials restrictions most industrial chemical processes are now carried out either at moderate pressure and high temperature or at high pressure and moderate temperatures.

Thermodynamically, the best option for reactions requiring high temperature or a combination of high temperature and pressure would be a reactor that performs an adiabatic compression/heating step, followed by the chemical reaction at high temperature and pressure, concluded by an adiabatic expansion step that recovers all of the energy put in for heating and compressing. Theoretically such a reactor would have no losses. 
This is the basic operating principle of the Pulsed Compression Reactor (PCR). The description of the working of the reactor, its advantages and drawbacks as well as an overview of the historical developments is given in chapter 2 .

\subsection{The application of the PCR}

The PCR promises to be a compact, versatile and energy efficient alternative for conventional chemical reactors. The versatility of the PCR allows it to be applied for a great number of processes. These include: endothermic and exothermic gas phase reactions, heterogeneous gas-liquid reactions and even gas-solid reactions (in form of ultrafine powders). Within the scope of this project, the production of syngas from methane is investigated. There are several reasons for choosing this application as one of the first ones to be investigated. In short, these include:

- The increase of energy efficiency and reduction capital cost of syngas-based products

- Development of a compact syngas reactor, greatly decreasing the footprint of syngas plants

- Reduction of the environmental impact of syngas as a bulk chemical

- Reduction of natural gas flaring

The PCR can be used for both endothermic and exothermic reactions. This means that steam- and carbon dioxide reforming, as well as partial oxidation of methane or a combination thereof is possible in the PCR. The PCR will have numerous advantages over conventional processes for syngas production.

Naturally there are quite a few alternatives to convert natural gas into more valuable products. The bulk of these are large scale processes, aimed at converting large amounts of natural gas (or just methane) to more valuable products. These processes include:

- Steam $/ \mathrm{CO}_{2}$ reforming of methane

- Partial oxidation of methane

- Autothermal reforming

- Combined reforming

The one aspect that these processes have in common is that all operate at temperatures over $1000 \mathrm{~K}$, and thus have a carbon efficiency of no higher than 75\% [4] and [5].

As mentioned, PCR technology is an emerging technology that will require going through some more development before it is industry ripe. The research presented in this thesis 
aims at moving PCR technology closer to the goal of applying the PCR for the conversion of methane. Methods and sub goals of various experiments will be presented in the corresponding sections. Details of simulations done will be explained and their results compared to experiments performed.

\subsection{Research questions and outline}

In order to assess the feasibility of the application of the PCR principle for the conversion methane to syngas, several fundamental questions need to be answered. Two important questions that relate to the applicability of the PCR for any process are: how large is the heat transfer rate from a rapidly compressed and expanded volume of gas, and how does this heat transfer rate compare to energy contained in the compressed gas? And: can stable operation with a completely free piston as it is intended with the PCR be achieved?

The most important question that needs to be asked is related to the feasibility of using the PCR for the conversion of methane is: will rapid compression of a mixture of methane and oxygen, or methane and steam, result in synthesis gas? And if it does, how will the yields of syngas compare to conventional reactors?

These three fundamental questions are the heart of the research presented in this thesis. To answer these questions the following research strategy is applied. Two setups were designed, built and applied for a range of experiments. These setups are described in detail in chapter 3 . They consist of a "continuous" reactor and a "single shot" reactor. In the continuous reactor, the heat transfer rate was measured. The measurement and data analysis method are described in chapter 3 , the results of the continuous heat transfer rate measurements are shown in chapter 4.

In the single shot reactor, both instantaneous heat flux measurements were performed, as well as different experiments aimed at investigating the chemistry that occurs in the PCR. The experimental and data analysis methods for these experiments are described in chapter 3. The results from the instantaneous heat flux measurements are given in chapter 4 . Results from the chemistry experiments are given in chapter 5.

Using the experimental results as a validation tool a numerical simulation method was developed for the PCR chemistry. This method is described briefly in chapter 5 , followed by a detailed comparison between simulation and experimental results. The simulations are then used to make a more in depth analysis of various aspects of the PCR: the 
different chemical processes that occur, a comparison of product to equilibrium compositions and an energy analysis of the formation of products.

The experimental data obtained is finally used to arrive at an analysis of the stability issue of the PCR. This is done in chapter 6.

Additionally, in chapter 2 some background information on the PCR is given: the advantages of the PCR over both conventional chemical reactors as well as internal combustion engines, different operating modes of the PCR and a short overview of previous work on PCR and comparable machines.

Last but not least, in chapter 7 the conclusions, including the answers to the formulated research questions are given. This is followed by some recommendations for further research. 



\section{The background of the PCR}

The functioning of the PCR can be compared to that of an internal combustion engine. The internal combustion engine is an efficient chemical reactor, but has some drawbacks that prevent it from being used on a large scale as an industrial reactor. The PCR is a reactor that possesses the advantages of the internal combustion engine, but averts some of its important drawbacks.

In this chapter firstly the advantages of the internal combustion engine are discussed. Then the reasons that the internal combustion engine is not used widely as an industrial chemical reactor are explained. This forms the basis for the elaboration on the functioning of the PCR and its advantages over both the internal combustion engine as well as conventional reactors. Lastly an overview is given of the different operating modes that the PCR can be operated in and a short historical overview of previous research on the PCR and related subjects.

\subsection{The internal combustion engine as a chemical reactor}

The internal combustion engine for mobile propulsion is a type of chemical reactor. It converts fuel and oxidizer to mostly carbon dioxide and water, generating shaft power at the same time. While the internal combustion engine is the most used chemical batch reactor in the world, it is hardly ever included in the list of main reactor types.

Compared to different types of commonly used chemical reactors in industry, specifically in terms of energy balance, the internal combustion engine seems to be a very attractive alternative. The most important advantages of the internal combustion engine, compared to conventional chemical reactors can be summed up as follows:

- Integrated compressive heating, reaction, expansion and quenching steps

- Energy recovery

- Homogeneous dense reactant mixtures, while at the same time keeping a low average reactor temperature

These advantages will be clarified in the following sections. 


\section{Integrated heating, compression, expansion and quenching steps}

The integration of many conventional process steps into one moving part makes the internal combustion engine a very efficient machine, without the need for investing into expensive energy recuperation equipment. The energy required for compression and heating of the reactant flow is, after reactions have taken place, immediately recovered and transferred to the next volume of reactants in a continuous process, where only the losses from the reactor have to be compensated.

\section{Energy recovery}

The immediate expansion of the hot reaction products recovers almost all of the energy initially put in, as well as most of the energy that is released in the chemical reaction. This energy is immediately used to compress the next batch of reactants as well as extracted from the reactor in the form of work. The quick succession of steps ensures that energy losses are relatively small. The integration of energy addition and recovery into one moving piston prevents the need of complicated energy recovery systems.

\section{Homogeneous dense reactant mixtures}

The compression of the reactant mixtures produces a dense, generally homogeneous reactant mixture (the homogeneousness of the mixture depends on the type of engine). The pressure and temperature of the reaction mixture is generally much higher than in conventional chemical reactors, allowing for faster reaction rates.

So if an internal combustion engine type reactor sounds so attractive, why are they not widely used as chemical reactors for a more wide range of chemical industrial applications?

Many attempts have been made to utilize internal combustion engines as chemical reactors, for example in the work by Kolbanovskii et al. [6], Von Szeszich [7] and Karim [8]. Alternative fuels such as hydrogen, vegetable oil, ammonia, hydrazine, coal-water and carbon slurries and many others have been tried [9]. However, the success of projects to use internal combustion engines to actually replace conventional reactors in industry has always been limited. The failing of the large scale application of conventional internal combustion engines as industrial chemical reactors can be ascribed to many factors, the most important of which will be outlined here:

- Limitations on maximum attainable pressures and temperatures

- Limitations on throughput

- Physical limitations associated with oil lubrication

- Chemical limitations associated with oil lubrication 


\section{Limitations on maximum attainable pressure and temperature}

The maximum attainable pressures and temperatures of the most advanced internal combustion engines are around 180 bar at the top dead centre and 700 to $900 \mathrm{~K}$ (not taking the temperature increase by combustion into account) with the highest compression ratio no larger than 1:25. This upper limit of pressure is even a limit for supercharged engines, where the air charge into the engine is already under pressure. To reach pressures like this with naturally aspirated engines one would require much higher compression ratios. Though new engines are always under development, and maximum pressures, temperatures and compression ratios are still rising, pressures and temperatures of this order are in fact still similar to pressures and temperatures already used in conventional chemistry. If one would like to have any serious benefit from employing IC's as chemical reactors the attainable pressures and temperatures would have to be significantly higher. This is, from point of view of the mechanics of various engine parts, not yet possible.

\section{Limitations on throughput}

The problem associated with throughput can best be seen by looking at the different limits of IC's. Comparing one very small engine, to one very big one as is done in Table 2.1, a trade off becomes apparent. While small engines can reach very high reciprocation rates, thus relatively high throughput of chemicals (relative to the size of the engine), their absolute production rate is very small. Larger engines can handle larger volume flows, but since they operate at much slower reciprocation rates, the relative volume flow (relative to their size) only decreases.

\begin{tabular}{ccc}
\hline \hline & Cox TEE DEE .010 & MAN B\&W K98MC \\
\hline Cylinder diameter & $6.02 \mathrm{~mm}$ & $980 \mathrm{~mm}$ \\
Stroke & $5.74 \mathrm{~mm}$ & $2660 \mathrm{~mm}$ \\
Displacement & $0.163 \times 10^{-6} \mathrm{~m}^{3}$ & $2.0 \mathrm{~m}^{3}$ \\
Operation rate & $533 \mathrm{~Hz}\left(32000 \mathrm{rpm}^{-1}\right.$ & $1.57 \mathrm{~Hz}(94 \mathrm{rpm})$ \\
Flow rate & $5.2 \times 10^{-3} \mathrm{~m}^{3} \mathrm{~min}^{-1}$ & $189 \mathrm{~m}^{3} \mathrm{~min}^{-1}$ \\
\hline \hline
\end{tabular}

Table 2.1, A very small engine compared to a very large one

\section{Physical limitations associated with oil lubrication}

Oil lubrication is still always an integral part of any internal combustion engine. Though new lubrication-free engines are under development, lubrication is currently still necessary for a range of reasons. The oil film between piston and engine wall reduces friction and guarantees a good seal between the piston and wall. Engine parts need to be protected from corrosion by the oil; since temperatures are high, reactive by-products from the combustion process will harm the engine quickly. 
The presence of oil limits the maximum operating temperature of the engine. Due to the limited thermal stability of the oils, the engine, or lubricated parts of reactor can never reach a temperature significantly higher than $200{ }^{\circ} \mathrm{C}$. Though new types of high performance lubricants are under investigation (like described by Hiroyuki Fukui [10] and later by Zhu, J. M. [11]), costs of these lubricants are too high to be employed extensively. Though this does not mean that the peak temperature of the internal combustion engine cannot be significantly higher than $200{ }^{\circ} \mathrm{C}$, its average temperature must remain below it. This implies that reactants and products cannot be preheated much, certainly not to temperatures above $200^{\circ} \mathrm{C}$, at least not without extensive cooling meaning energy losses. The lower (often ambient) starting temperature directly limits the highest temperature reached at the peak of compression, limiting the effectiveness of the internal combustion engine as a chemical reactor.

\section{Chemical limitations associated with oil lubrication}

The three restrictions previously described concern physical limitations of temperature, pressure and throughput. There are however also some chemical limitations. Every today's lubricating oil is a multi component mixture of chemical compounds. As a result a number of chemical processes, especially catalytic ones, cannot be carried out in presence of oils. Oil itself and products of its destruction can harm downstream processes. In turn, lubricating oils cannot withstand highly reactive atmospheres of numerous chemical processes. For example, a presence of oils in pure oxygen or chlorine can result in an explosion. Any changes in chemical nature of an air-fuel charge in IC engines may drastically change a lubrication pattern - for example application of alcohols instead of gasoline as fuel involves serious wear problems [12], [13]. Generally every new fuel requires appropriate new oil, and the same will hold for every chemical process.

\subsection{The pulsed compression reactor}

The Pulsed Compression Reactor does not share the drawbacks that prevent the internal combustion engine from being used widely as an industrial reactor. This makes the PCR a much more likely candidate for the replacement of conventional chemical reactors.

\subsection{Reactor principle}

The principals of the PCR were proposed over eighty years ago as can be seen from patents by Brutzkus [14] and the book by Kolbanovskii et al. [6]. None of these reactors were successful. Further work by Glushenkov solved some critical problems with the proposed designs, allowing for successful implementation [15]. The reactor promises to be a very compact, economical and energy efficient alternative to conventional chemical 
reactors used for the most energy consuming high temperature processes. The PCR is a free piston impulse compression device. It rapidly compresses the reactants by a free piston reciprocating inside a cylinder. Due to the compression the reactants are heated and spontaneously react. A schematic view of the basic PCR design as proposed by Glushenkov is shown in Figure 2.1. Reactants are injected, displacing products, through ports in the side of the reactor, when the piston is near one of its extreme positions. The actual design of the PCR can be much different from the design shown in Figure 2.1, depending on the application.
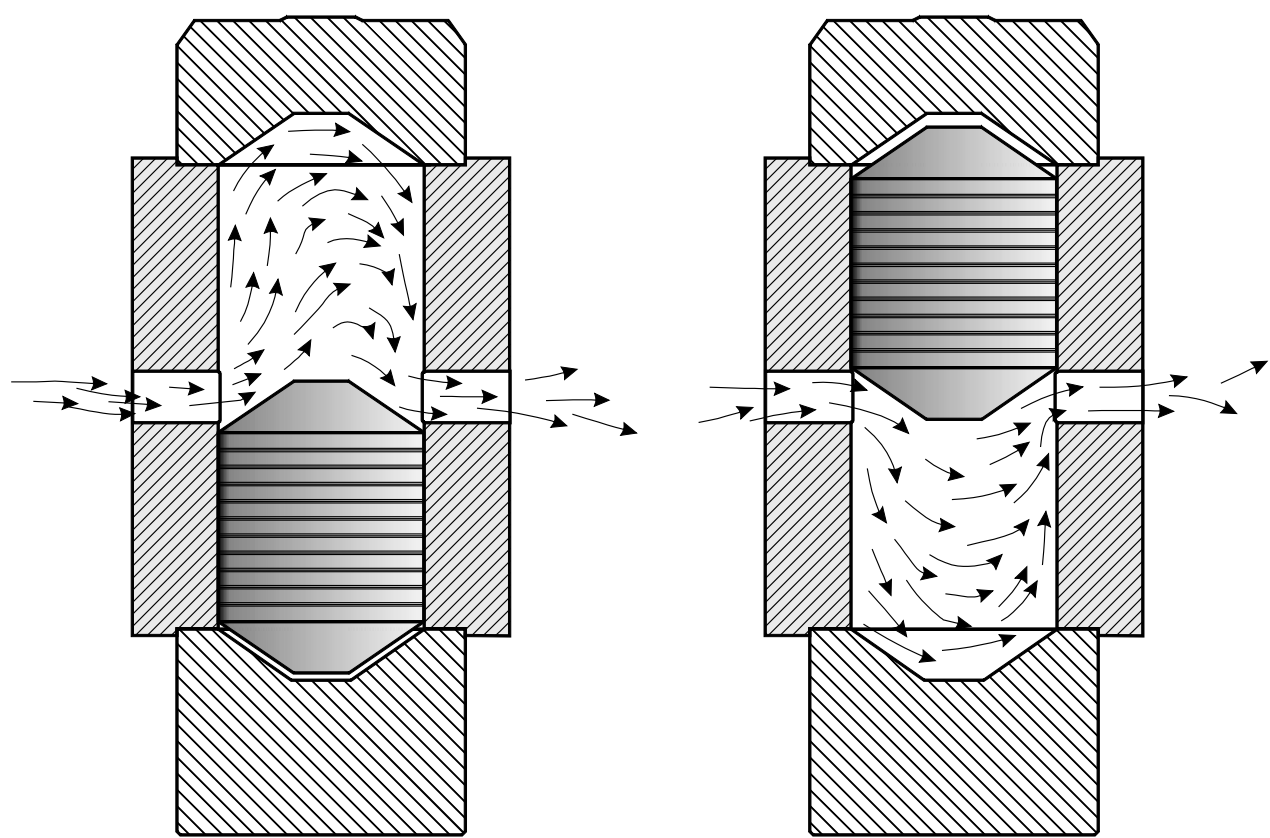

Figure 2.1, Schematic view illustrating the PCR principle

The functioning of the PCR can be compared to that of an internal combustion engine, with some important differences. Where most conventional engines use crank gears, piston rings, lubricating oil and relatively low operating frequencies and pressures, the PCR uses clearance sealing with labyrinth grooves, gas lubrication and higher operating frequencies and pressures. This means that during operation the piston does not touch the wall but is separated from the cylinder wall by a gas film.

This means that on top of enjoying all the benefits that the internal combustion engine offers, the PCR has some additional advantages. These can best be summarized as follows and will be discussed each individually. The advantages are subdivided in 
advantages the PCR has over conventional reactors and advantages compared to the internal combustion engine-like reactors.

- Advantages over the internal combustion engine as a reactor

- No lubrication oil required

- Less limits on operating frequency

- Higher attainable peak compression pressure

- Less limits on in- and outflow pressure

- Less scale up limitations

- Advantages over conventional reactors

- Energy efficiency

$\circ \quad$ High rate of heating and quenching

- Simple, compact design

- The unlocking of a new high pressure-temperature operating regime

- Tremendous reduction in material restrictions

- Optional functionality as a linear alternator

\section{No lubrication oil required}

In contrast to conventional internal combustion engines, as well as the most commonly used free piston engines, the sealing of the reaction chamber is done by clearance sealing with labyrinth grooves. This allows for gas lubrication of the piston, having (among others) the advantage that no lubrication oil is required. This expands the versatility of the type of chemical process that is performed in the reactor is obtained, without the need to develop and apply different types of lubricating fluids for each process.

\section{Less limits on operating frequency}

The absence of crank gears and oil lubrication and the use of gas lubrication, allows for much higher operating frequencies than conventional internal combustion engines, without significant wear. The high operating frequency results in a higher throughput of chemicals per reactor volume per unit of time.

\section{Higher attainable peak compression pressure}

The compression pressure that can be reached with the PCR is significantly higher than for internal combustion engines, thanks to the absence of crank gears and piston rings. This means that the compression temperature can also be higher without needing to preheat reactants more and that some chemical processes might not be possible to perform in a conventional internal combustion engine may be performed in the PCR. Additionally, higher compression pressures and temperatures may accelerate the 
chemical reaction rates to near instantaneous, giving another boost to the rate of throughput in the PCR.

\section{Less limits on in- and outflow pressure}

Since the attainable compression pressure is higher with the PCR, the initial pressure of the reaction mixture (inflow pressure) can also be higher. For example, increasing the inflow pressure of reactants from ambient to 20 bar, increases the throughput of the reactor twentyfold. An additional advantage of this increase in operating pressure is the output pressure will also increase. This avoids additional compression steps of the product, since many customers of, for example, modern $\mathrm{H}_{2}$ plants require the product at high pressure [16].

\section{Less scale up limitations}

Next to the already mentioned options of scale up through an increased operating pressure, the reactor can also be simply scaled up by increasing the reactor bore diameter. While the reactors that are typically used in the research done within this project have a bore of around $60 \mathrm{~mm}$, this is expected to be, for industrial applications, upgradable to around $300 \mathrm{~mm}$. This drastically increases the throughput of the PCR. The scale up of the reactor in terms of reactor bore diameter is limited by the scavenging rate of the reactor. One cannot keep increasing the reactor bore diameter limitlessly: at some point it will be impossible to efficiently scavenge the reaction chamber each reciprocation. Simple scale up beyond this point can be achieved by running multiple reactors in parallel.

\section{Energy efficiency}

The integrated recuperation of compression and heating energy means that, theoretically, no energy is lost during the process. This makes the reactor much more efficient than conventional reactors.

\section{High rate of heating and quenching}

The operation mode of the PCR, with a free piston reciprocating between two gas springs, makes that the rate at which reactants are heated and products quenched, can be as high as $10^{7} \mathrm{Ks}^{-1}$ [17]. This has two important advantages. Firstly it reduces the energy lost to the environment by making the cycle time very short, thus nearly adiabatic. Secondly, the high quenching rate means that reactions can be quenched in a non-equilibrium state, allowing for reactions to be "frozen" at the moment of maximum conversion to the desired product. 


\section{Simple, compact design}

The simple, compact design of the PCR, with only one moving part, makes that the PCR has a very low level of capital investment costs. The gas lubrication of the piston keeps wear to a minimum, making the maintenance costs minimal also. The compactness of the design, even for scaled up reactors, adds greatly to the mobility of the reactor and the employability in remote areas such as offshore drilling rigs or in the vicinity of small gas resources for GTL applications.

\section{The unlocking of a new high pressure-temperature operating regime}

The PCR unlocks a new regime of operation with a combination of high temperatures and high pressure that cannot be achieved in conventional reactors. This opens the doors for new or modified processes that would not be possible in conventional reactors.

\section{Tremendous reduction in material restrictions}

While the peak compression temperatures and pressures of the reactant gas that can be reached in the PCR are orders of magnitude higher than in conventional reactors, the reactor temperature is much lower. This means that while temperatures and pressures that cannot be reached in conventional reactors are attained, the restrictions on the materials used are tremendously lower.

\section{Optional functionality as a linear alternator}

The PCR can be equipped with a linear alternator to extract electrical power from the reactor during operation, in the case of highly exothermic reactions. The extraction of electrical power may even be used as a control tool on the PCR. While the functionality of the PCR as a power generating device on top of being a chemical reactor is an interesting line of research, it is not further pursued in this project.

\subsubsection{The principle of compressive heating}

The leading principle that makes both internal combustion engines, as well as the PCR work is the principle of compressive heating. When an ideal gas is compressed quickly, its temperature and pressure will rise. If the compression is done quickly enough that no energy is exchanged between the gas and the surrounding walls the temperature, pressure and volume change are related to each other according to the adiabatic compression relations:

$$
\begin{aligned}
& P V^{\gamma}=\text { const }, \\
& \frac{P^{\frac{\gamma-1}{\gamma}}}{T}=\text { const },
\end{aligned}
$$




$$
T V^{\gamma-1}=\text { const } .
$$

The parameter that determines the various adiabatic relationships is the specific heat capacity ratio (adiabatic constant), which is different for different gasses. In Figure 2.2 the temperature as a function of compression ratio is shown for two gasses, at two different initial temperatures.

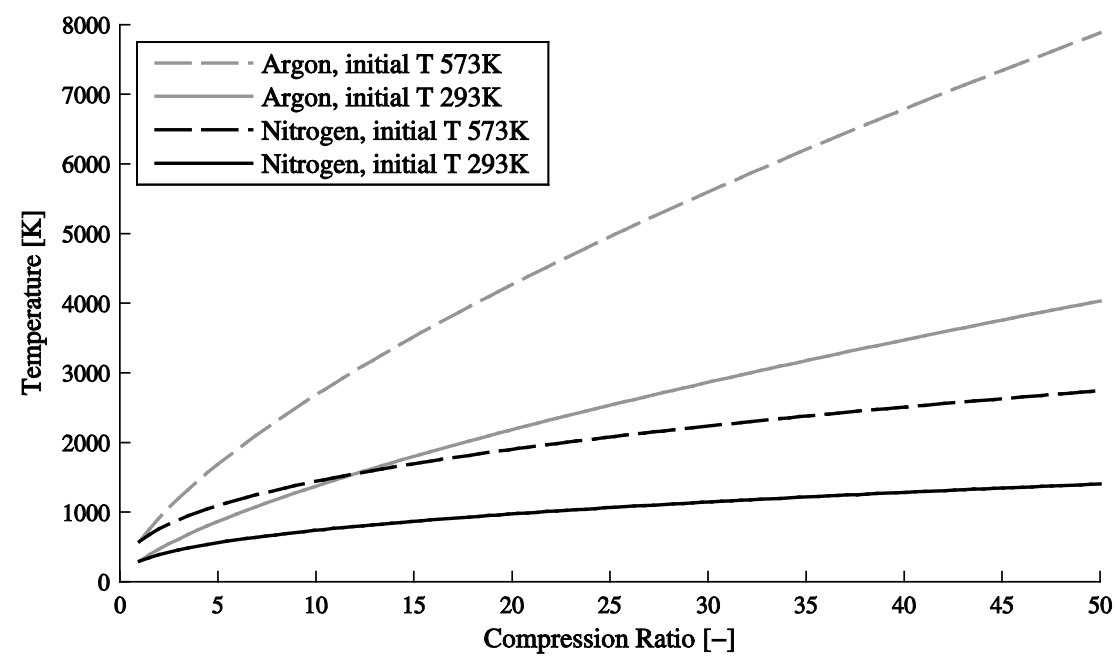

Figure 2.2, Temperature as a function of compression ratio for Nitrogen and Argon

The temperature of the gas with a higher specific heat capacity ratio becomes higher for the same compression ratio (the ratio between original volume and compressed volume). The increasing of the initial temperature by a certain factor also increases the final compression temperature (at the same compression work applied) by the same factor.

Figure 2.2 as well as equations 2-1 through 2-3 only apply to ideal gasses. When compression ratios get much higher than the compression ratio's presented in Figure 2.2 , non ideal behavior of the gas will start to influence the results, and equations 2-1 through 2-3 will no longer apply.

\subsubsection{Different operation modes}

There are several operating modes possible for the PCR, some of which are investigated within this project. The different operating modes are classified as follows and will be discussed in more detail individually: 
- Normal dual chamber mode

- Separate feed dual chamber mode

- Driven single chamber mode

- Non-driven single chamber mode

\section{Normal dual chamber mode}

The schematic diagram that was shown previously in section 2.3 (Figure 2.1) shows the reactor operation as it would in normal dual chamber mode. In this mode, both reaction chambers are used for chemical reactions. Both reaction chambers are fed by the same feeding channels. The length of the piston is taken in such a way that when it is near one of its extreme positions it unblocks the in- and outflow openings to the uncompressed chamber. This allows the reactants to flow in and products to flow out from the uncompressed chamber.

\section{Separate feed dual chamber mode}

The separate feed dual chamber reactor is a variation on the normal dual chamber reactor. The difference lies in the number of in- and outflow openings the reactor has. While the normal dual chamber reactor has only one set of in- and outflow openings to feed both reaction chambers, the separate feed dual chamber reactor has two sets of inand outflow openings. The piston length is chosen such that when the piston is near one of its extreme positions it unblocks one set of in- and outflow openings, that connect to the uncompressed chamber. When it is near the other extreme position, it unblocks the other set of openings, connecting to the other chamber. This operation is shown schematically in Figure 2.3.

The advantage of this reactor design and operation mode is that different reactions can be performed in the different chambers. For example, one could perform an endothermic reaction in one of the chambers and an exothermic reaction in the other chamber. The endothermic reaction will withdraw energy from the system during each reciprocation, while the exothermic adds energy every reciprocation. If the reactions are selected and tuned properly, this will equilibrate the net energy balance. Of course a reactor that is suitable for separate feed dual chamber mode can also be used in normal dual chamber mode by simply supplying the same feed to each of the reaction chambers. 

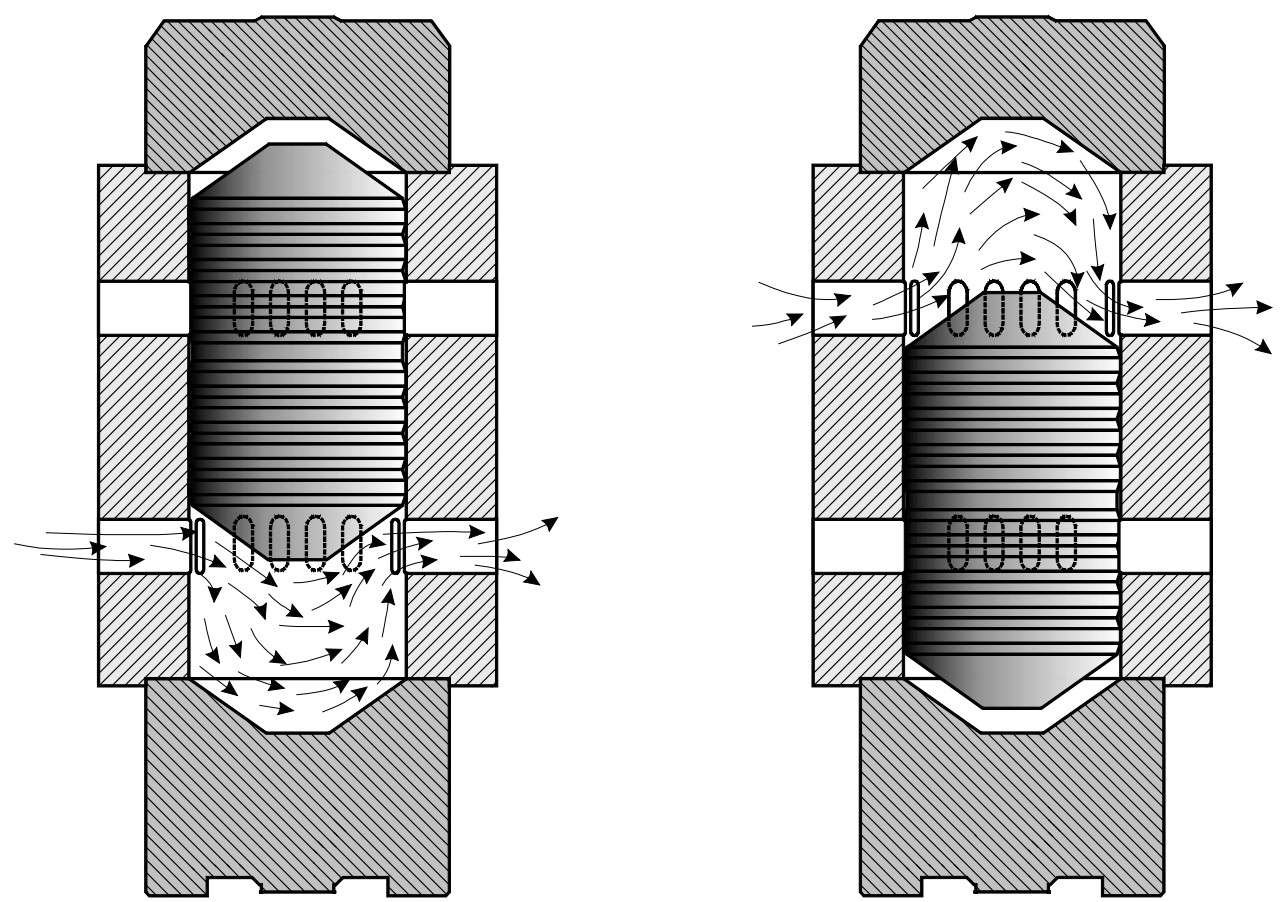

Figure 2.3, Schematic view illustrating the PCR separate dual chamber mode

\section{Driven single chamber mode}

The driven single chamber mode is a mode that can be performed with a similar reactor as is shown in Figure 2.3. In the driven single chamber mode, compressed gas is supplied through a valve in the lower cover of the reactor. This compressed gas helps sustain the reciprocation of the piston (the phenomena known as the pneumatic hammer), while reactions are made to occur in the upper chamber. The reactions in the upper chamber can be both endothermic as well as exothermic (one simply needs to adjust the amount of energy that is supplied by the compressed driving gas in the lower chamber).

\section{Non-driven single chamber mode}

Once reciprocation is achieved and (exothermic) chemical reactions occur in the upper chamber through driven single chamber mode, the valve through which driving air is supplied can be closed in order to achieve non-driven single chamber mode. In this mode no driving gas is supplied to the lower chamber, but no chemical reactions occur there either: the lower chamber is simply used as a bounce chamber (gas spring). This mode is only possible with exothermic reactions in the upper chamber. 


\subsubsection{Previous work}

Previous work on free piston devices can roughly be divided into four main areas. Firstly there is the work done in the field of PCR technology that preceded the project that this research is part of. Secondly, free piston engines have been developed and produced between fifty to seventy years ago. The third source of information is attempted applications of internal combustion engines as chemical reactors. Lastly there is the application of free piston ballistic compressor like devices for the investigation of chemical kinetics. In the following sections short overviews will be given of some available literature (with special emphasis on the literature that most closely relates to the research done within this project) of each of these areas.

\section{Previous work on PCR technology}

The first fundamentals of pulsed compression technology can be found in literature as early as the 1920s. For example the patent by Humphrey in 1922 [18] and later one by Brutzkus in 1926 [14]. Later patents on chemical reactors utilizing the rapid compression principle include two by van Dijk in 1957 [19], [20] and several by Kolbanovskii in 1975, 1978,1986 and 1988 [21]. A patent that specifically mentions the production of syngas by compression is the one by Herwig [22].

While the concepts laid down in the patents are similar to, or at least share some of the properties of, PCR technology, none of the described technologies have even been realized in practice. The reason is that the reactor concepts in the mentioned patents did not solve four crucial problems, namely reactor start-up, stable piston reciprocation, wear-free (or at least low wear) operation and sealing of the piston-cylinder clearance.

\begin{tabular}{cc}
\hline \hline timeframe & Achievements \\
\hline 1995-1998 & $\begin{array}{c}\text { Development of a free-piston reciprocation device and several start- } \\
\text { up systems. Demonstration of self-sustaining operation. [15], [23] }\end{array}$ \\
\hline 1998-2001 & $\begin{array}{c}\text { Development of start-up systems with no moving part. Establishing } \\
\text { principles of stable device start-up and its operation without chemical } \\
\text { reactions. }\end{array}$ \\
\hline 2001-2002 & $\begin{array}{c}\text { Achieving very high compression ratios (45) and frequency of piston } \\
\text { reciprocation (up to 200 Hz). Establishing principles of selection of } \\
\text { materials for pistons and cylinders. [24] }\end{array}$ \\
\hline 2002-2007 & $\begin{array}{c}\text { Construction of the first pilot plant and two reactors for carrying out } \\
\text { non-catalytic chemical reactions. Demonstration of the feasibility of } \\
\text { syngas production by partial oxidation of liquid and gaseous } \\
\text { hydrocarbons in short runs (30 - 60 s). [17], [25], [26], [27], [28] }\end{array}$ \\
\hline \hline
\end{tabular}

Table 2.2, Overview of historical developments of the PCR technology 
Solutions to these problems were first proposed by Glouchenkov in 1997, as can be seen from his patents [15]. The first justification for proposed the technology was given in a manuscript registered in the Russian society of intellectual rights [23]. An overview of the development of the PCR from this point onwards is given in Table 2.2.

While the fundamentals of PCR technology have been laid down long ago, the real development of the PCR as it is investigated within this project has only begun relatively recently. This means not much has been published on PCR technology itself. However, similar developments, such as the development of free piston engines and the use of free piston ballistic compressors for chemical kinetics investigations have been going on much longer, so much more publications can be found in this field.

\section{Free piston engines}

Free piston engines have been developed and used from the 1950s until today, for a variety of applications. Early publications on free piston engines, such as [29], describe the application of free piston engines in automotive, locomotive and maritime applications.

While on the surface a free piston engine may seem like a very similar device to a PCR, there are two important differences. Firstly, most free piston engines, like those used in the work of Mikalsen et al [30] and by Xu et al [31], are based on conventional engine technology. So they use piston rings and lubrication oil. A second important difference is that the main purpose a free piston engine is to perform work. This means that a load is present. So, in contrast to PCR's, a degree of control over the piston motion in a free piston engine can be obtained through varying the load (the amount of work delivered by the free piston engine).

Potential advantages of the free piston engine include optimized combustion through variable compression ratio, leading to higher part load efficiency and possible multi-fuel operation, as well as reduced frictional losses due to a simple design with few moving parts [32]. While the main goal of free piston engines may be different from that of the PCR research done within this project, there are still some similarities in the type of research done.

Free piston engine research can roughly be divided in three areas. These are:

- Research on different designs and engine layouts

- Research on engine control

- Research on combustion kinetics and modeling 
An example of research on different engine designs and layout is the engine concept put forth by Aichlmayr et al [33]. An overview of the different free piston engine concepts that are in existence is given by the article by Achten et al [34].

There are many sources describing new ways of free piston engine control and the relation between combustion efficiency and control. Examples of these are the work by Johansen et al on free piston diesel engine control [35], the work by Zulkifli et al on starting by mechanical resonance [36] and more general work on free piston engine control by Mikalsen et al (in two parts) [37], [38].

The research that has perhaps the most in common with the work done within this project on PCR is the research on combustion kinetics and modeling. A very simple simulation model of free piston engine motion, similar to the models used for modeling the PCR in this thesis, is presented in the work by Mikalsen et al [39]. A combination of experimental characterization and the use of kinetic models for a free piston engine is presented in the work by Aichlmayr et al [40].

\section{Internal combustion engines as chemical reactors}

The attempts to apply internal combustion engines as chemical reactors for other purposes than power generation are numerous. The most recent and possibly the most applicable to this research is the work by Karim, who used an internal combustion engine to produce hydrogen by partial oxidation of methane [41]. An overview some different patents acquired over the last century that attempting to utilize the internal combustion engine for various chemical processes is shown in Table 2.3.

\begin{tabular}{ccc}
\hline \hline Year & Type of reactor / process & $\begin{array}{c}\text { Patent number / } \\
\text { reference }\end{array}$ \\
\hline 1918 & Production of nitric oxide & US pat 1283112 \\
\hline 1944 & Partial oxidation of solid fuels & US pat 2363708 \\
\hline 1947 & Oxidizing hydrogen sulfide & US pat 2415904 \\
\hline 1951 & Generation of syngas & US pat 2543791 \\
\hline 1952 & Generation of syngas & US pat 2591687 \\
\hline 1961 & Fixation of nitrogen & US pat 2977938 \\
\hline 1979 & Production of hydrohalic acids & US pat 4154811 \\
\hline 1987 & Combustion of toxic waste & US pat 4681072 \\
\hline 1989 & General application for gas phase reactions & US pat 4816121 \\
\hline \hline
\end{tabular}

Table 2.3, Overview of various patents utilizing the internal combustion engine as chemical reactor 


\section{Free piston ballistic compressors for chemical kinetics investigations}

The number of experiments and groups around the world that have used shock tubes for the investigation chemical kinetics is very large. Many publications can be found on a great variety of chemicals and shock tube configurations. A selection of literature on chemical conversion by compression is presented; presenting those articles that are most closely related to the work done within this project is shown in Table 2.4.

\begin{tabular}{cl}
\hline \hline Type of research & \multicolumn{1}{c}{ References } \\
\hline Kinetics in rapid & $\begin{array}{l}\text { Design of a rapid compression machine for chemical } \\
\text { investigations: Mittal et al [42] }\end{array}$ \\
& $\begin{array}{l}\text { Application of a rapid compression machine for the } \\
\text { investigation of ignition of syngas mixtures: Mittal et al [43] }\end{array}$ \\
& $\begin{array}{c}\text { Application of a rapid compression machine for the } \\
\text { investigation of ignition of methane/hydrogen mixtures: } \\
\\
\text { Gersen et al [44] }\end{array}$ \\
\hline $\begin{array}{c}\text { Production of } \\
\text { hydrogen or syngas by } \\
\text { rapid compression }\end{array}$ & $\begin{array}{l}\text { Conversion of methane to acetylene or syngas: Kado et al [45], } \\
\text { Syngas production in an internal combustion engine: McMillan } \\
\text { et al [47], Yang et al [48] }\end{array}$ \\
\hline \hline
\end{tabular}

Table 2.4, Short overview of literature available on free piston ballistic compressors experiments related to $P C R$ research 



\section{The experimental setup}

A large part of the research done within the framework of this project is concerned with the design, testing and use of various experimental setups and measurement methods. In the following chapter, the different experimental goals, with their corresponding experimental setup designs are explained. Also the different experimental methods and methods of data analysis are addressed. Methods and procedures are, where necessary, illustrated by example measurements.

\subsection{Introduction}

For the further development of the PCR, it is of vital importance to develop good experimental setups and methods to acquire data that helps further develop the technology and cure child diseases. Within the scope of the research presented in this thesis, the measurement goals of the experimental setups can be subdivided in the following parts, which will be clarified further in the subsequent sections.

- Heat transfer

- Instantaneous heat loss

- Continuous heat transfer

- Chemistry

- Partial oxidation:

- Initial temperature

- Piston kinetic energy

- Reactant mixture composition

- Piston mass

- Flame quenching

○ Steam reforming

- Initial temperature

- Piston kinetic energy

\subsubsection{Heat transfer}

In steady operation, a balance between the energy lost from the gas, energy put in and energy produced or consumed by chemical reactions will be established. At this steady 
operation point, the temperature will oscillate steadily around a constant value. This constant mean temperature depends on the heat transfer rate, net energy release by chemistry and the external energy input.

The clearance sealing of the piston-cylinder gap is greatly sensitive to temperature changes, especially if the cylinder and piston are made of materials with a different thermal expansion coefficients. For this reason, it is very important to know beforehand what the steady operation temperature of the reactor will be, so the piston-cylinder combination can be designed with minimal clearance at steady operation.

At the same time, the energy release of the chemical reactions will need to be tuned to achieve a steady operation mode, which has a desired product composition. So, in order to be able to predict this steady operating temperature as a design parameter, as well as facilitate the tuning of the chemical reactions to a desirable product composition, some knowledge will have to be gained on the heat transfer rate in the PCR.

In the PCR, many types and sources of losses can be identified. These include, but are not limited to:

- Heat transfer from the hot compressed reaction chamber to the reactor walls and piston

- Compressed gas losses through the gap between piston and cylinder

- Hydraulic losses in the in- and outflow openings (so-called pumping losses)

- Friction losses between piston and cylinder

Of these different losses, the first will be considered in this thesis. The reason is that this loss has, potentially, the largest implications on the success of the PCR as a chemical reactor. While hydraulic losses in the in- and outflow openings can be quite significant, they may be partly overcome by increasing the pressure of reactant injection and removal, thus increasing the external energy input. The other losses are "internal" energy losses and cannot be compensated for by external energy input (at least not when the reactor runs in dual chamber mode as described in section 2.3.3 ). Of these internal energy losses, the friction losses between piston and cylinder are expected to be small compared to the energy contents of the system. The main reason for this is that significant friction losses (beyond the shear forces within the gas between the piston and cylinder) will only occur if a large side force is acting on the piston. Since the whole reactor is symmetric and mounted vertically, these side forces are expected to be quite small. 
The effects of heat transfer on the other hand can be disastrous. If heat losses are too high, the cooling effect which the walls have on the hot compressed reaction mixture may slow down or even totally prevent chemical reactions.

Within this research a distinction is made between average heat transfer measurement, and instantaneous heat flux measurement. Their definitions, as they are used within this research are as follows.

\section{Average heat transfer measurement}

The average heat transfer measurements have a spatial- and temporal resolution in such a way that the measurement does not capture heat flux variations within one cycle. Instead, the measurement looks at the average heat transfer between the gas and the reactor wall, averaged over a number of cycles. To measure the average heat transfer the demands on measurement devices, location and sample speed are not so strenuous.

\section{Instantaneous heat flux measurement}

In contrast to the average heat transfer measurement, the instantaneous heat flux measurement is done in such a way that the spatial and temporal resolution are high enough to capture the oscillations of the heat flux during one cycle of the reactor. This means that the demands on the sensor response time, sensor size and sample rate are significantly higher. Also the demands on data processing accuracy are somewhat more strenuous. On the other hand, the size of the temperature difference is also bigger, making the problem of noise within the measurement a bit smaller.

\subsubsection{Chemistry}

The concept of the PCR is a very novel one, and one that has not been investigated in great detail in the past. Most comparable studies that have been done, on various chemical processes, have been limited to the adaptation of internal combustion engine like concepts (see section 2.3.4). At the same time, the combination of high temperature high pressure reached in the PCR cannot be reached in other reactors. This means that very little is known from literature about the chemistry that may occur under the extreme circumstances as they appear in the PCR.

While the PCR is a very versatile device, which can be used for a multitude of chemical applications, the research in this thesis has been limited to one specific bulk process: the generation of synthesis gas from methane. This is done both by partial oxidation with air, as well as by steam reforming. 
The experimental studies performed within this thesis aim at giving insight into the chemistry and effectiveness of syngas production from methane by means of PCR. Within that goal an attempt is made to isolate several parameters, and study their effect on the chemistry and effectiveness of syngas production. These parameters include: piston kinetic energy, preheat temperature, reactant mixture composition, piston mass and reaction chamber geometry.

\subsubsection{Continuous operation versus single shot}

Within the scope of this project a variety of experiments were planned. Some of these experiments could only be performed in a continuously operated PCR, similar to the principle outlined in 2.3 . For others it was more advantageous, from a parameter isolation point of view, to perform the experiments in a single shot reactor. By this is meant a reactor that mimics one single compression expansion cycle of the continuous PCR reactor. Each of the two setups will be discussed separately, with the different experimental methods and data analysis procedures developed.

\subsection{The continuous PCR experimental setup}

\subsubsection{Introduction}

The importance of having an accurate, robust method for measuring the heat transfer rate from gas to the wall, whether it be an average- or instantaneous heat flux, has become clear from section 3.1.1. The dramatic influence on the performance of the PCR that losses can have dictates that good measurement techniques are desirable. In light of this, the goals for the experiments in the continuous PCR are as follows:

1. To develop an accurate and robust method for measuring the average transfer rate from the gas to the reactor walls and piston, for different operating conditions.

2. To test this method by measuring the averaged heat flux at various operating conditions and using this flux to calculate a heat transfer coefficient.

3. To find an empirical correlation between the heat transfer coefficient and the peak compression pressure in the reactor.

\subsubsection{Experimental setup}

The description of the experimental setup can best be divided in the description of five main parts. These will be discussed separately:

- The reactor

- Thermocouples 
- Pressure transducers

- Air supply system

- Data acquisition system

\section{The reactor}

The reactor used in the continuous PCR experimental setup was designed and built by Energy Conversion Technologies BV. In order to achieve the goals set for the averaged heat transfer measurements, the PCR reactor was run by supplying a continuous stream of air into the lower chamber. This serves both the purpose of starting the piston motion, as well as sustaining the motion during operation. This operating mode is schematically shown in Figure 3.1, and is similar to the principle of a pneumatic hammer.
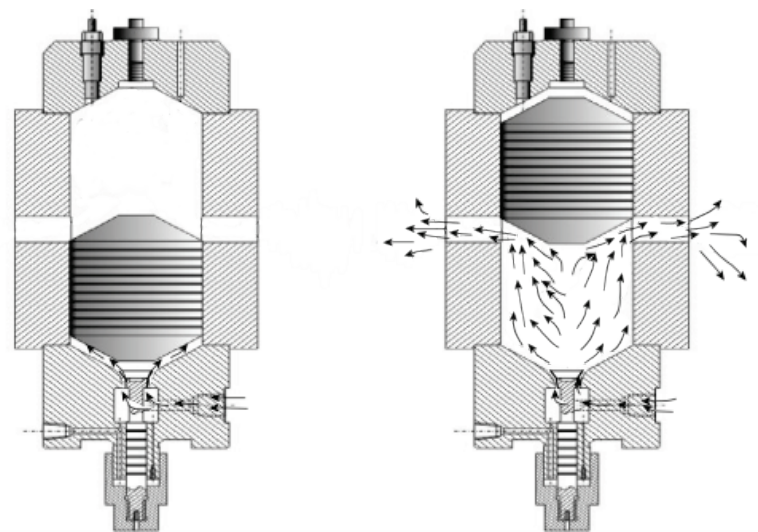

Figure 3.1, Schematic view of the running mode of the reactor for the average heat flux measurements

For the averaged heat transfer measurements this operating mode is used, in the absence of chemical reactions. So the reactor is simply run by supplying compressed air to the lower chamber, and compressing air in the upper chamber. There were several reasons that no chemical reactions were performed in the upper chamber. Firstly, performing chemical experiments in the setup would add a multitude of variables that would influence the heat transfer that would have to be isolated. These include, the reactant concentrations and type, injection rate, reactant preheat temperature and degree of mixing. Secondly, and possibly more important: the presence of chemical reactions does not have any additional value to the value of the results obtained, in light of the goals set. If an accurate and robust measuring method is developed, this method can always be applied to a PCR working with chemical reactions in a later stage. 
The reactor was constructed of stainless steel with a piston made of graphite. Different dimensions of the reactor and the mass of the piston are shown in Table 3.1.

\begin{tabular}{cc}
\hline \hline Bore diameter & $60 \mathrm{~mm}$ \\
Bore length & $135 \mathrm{~mm}$ \\
Piston length & $60 \mathrm{~mm}$ \\
Piston mass & $0.236 \mathrm{~kg}$ \\
\hline \hline
\end{tabular}

Table 3.1, Characteristic sizes and weights of the continuous reactor

\section{Thermocouples}

For the heat transfer measurements, a cover was designed and manufactured. Thermocouples at different depths were built in, for the purpose of the averaged heat transfer measurements. In Figure 3.2 a schematic cross section of the reactor with cover is shown, and in Figure 3.3 the design of the top cover is shown. In Figure 3.2 the various parts of the PCR used are shown: the air injection system, the pressure transducers, the piston, the inlet/outlet openings and the top cover. In the more detailed figure of the top cover, Figure 3.3, the holes for thermocouples are visible. These holes, five in total, are bored into the cover. The holes are bored to different depths, in a circular pattern, each at the same radial position from the centerline of the cover. The holes were $2.0 \mathrm{~mm}$ in diameter.

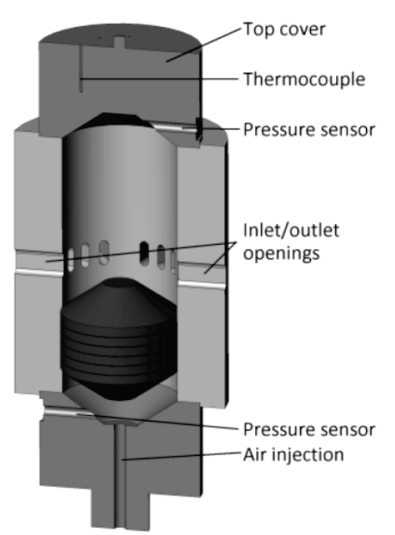

Figure 3.2, Schematic view of the PCR used for average heat flux measurements

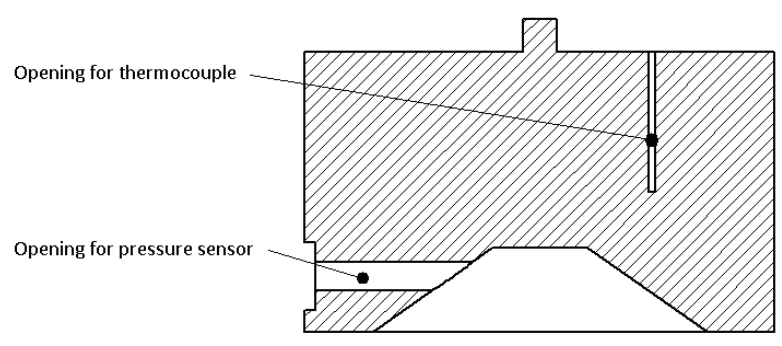

Figure 3.3, Design of the upper cover for average heat flux measurements

In these holes, thermocouples were inserted. To enhance heat transfer between the thermocouple and the cover, a conductive paste was injected into the holes prior to thermocouple insertion. The thermocouples were $1.0 \mathrm{~mm}$ K-type. For the purpose of 
later analysis, the distance that the thermocouples are placed from the hot compressed gas needs to be known. The distance that is taken is the distance perpendicular to the slanted surface of the cover of the reactor, as shown in Figure 3.4. The distances of the different thermocouples to the surface are shown in Table 3.2.

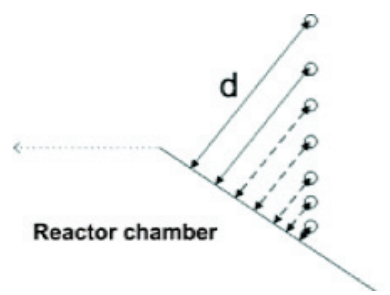

Figure 3.4, Thermocouple location with respect to the hot gas

\begin{tabular}{lccccc}
\hline \hline Thermocouple \# & 1 & 2 & 3 & 4 & 5 \\
Distance from surface $(\mathrm{mm})$ & 6.5 & 10.6 & 14.7 & 18.8 & 22.9 \\
\hline \hline
\end{tabular}

Table 3.2, Thermocouple distance from surface

In the design of the setup, a decision had to be made with regard to the depth that was chosen for the thermocouples. The following analysis was used as a basis for this decision.

The simplest way to measure the heat flux through an area of interest, is by measuring the temperature difference between two points in the material. The measured temperature gradient can be used to calculate an average heat flux through the space between the two measurement points, in the direction of the line connecting the two measurement points. There are some important considerations concerning measuring the heat flux in this way, which can most easily be explained at the hand of Figure 3.5.

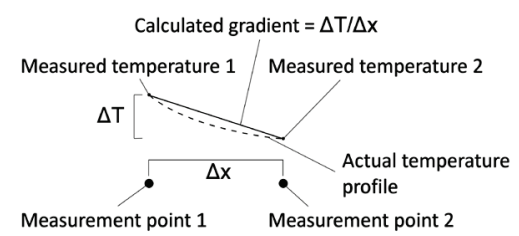

Figure 3.5, Schematic view of a heat flux measurement by two temperature probes

- The spatial resolution of the heat flux measurement is determined by the distance between the two measurement points and how accurately this 
distance can be determined. It is also limited by the size of the measurement device used.

- The temporal resolution is determined by one of two factors: either by the rate at which the temperature is measured, or by the time it takes for heat to travel from the first measurement point to the second, whichever is slower. The characteristic time for the heat propagation from one point to the other can be determined by the Fourier number:

$$
F_{o}=\frac{\alpha t}{L^{2}} .
$$

Taking that the Fourrier number must be at least larger than unity for heat to have propagated a length $L$, the characteristic time becomes:

$$
t=\frac{L^{2}}{\alpha} .
$$

- The accuracy of the heat flux measurement is also greatly influenced by the knowledge of the material between the measurement points. If this material contains much inhomogenety, this will influence the heat flux calculation in the accuracy of the thermal conductivity constant needed.

- The size of the temperature difference between the two measurement points needs to be sufficiently large. There is a certain degree of uncertainty as well as measurement noise when measuring the temperature, so the temperature difference between points needs to be large enough for this measurement uncertainty not to have a too great influence. One way to compensate for this effect is to put multiple thermocouples at the same depth, and average the measured temperatures.

\section{Pressure sensors}

In addition to the temperature measurements with thermocouples, pressure measurements were done with high speed pressure sensors in both chambers (Optrand D53294). The pressure measurements were needed to be able to compare different operating conditions of the reactor and to draw conclusions on the influence of different compression ratios on the heat losses. The pressure sensors had a working range of 0 340 bar, which was much more than required for the experiments.

\section{Air supply system}

To sustain the motion of the piston, pressurized air needed to be supplied continuously to the lower chamber. This was done by two different systems. For experiments at low 
pressures (static air supply pressure below 7 bar) the air was taken from the main laboratory air supply, which is kept at a constant 7 bar. From there it was reduced by a reducer to the desired pressure. For higher pressure experiments, air was taken from a 250 I storage tank, which was pre-charged to 150 bar. From there it was reduced by a high flow reducer to the desired pressure.

\section{Data acquisition}

The data from the thermocouples were acquired using LabView and a National Instruments NI-9213 data acquisition module, with a sampling frequency of $1 \mathrm{~Hz}$. Although LabView contains a standard K-type conversion of measured voltage to temperature, the used thermocouples were individually calibrated to reduce error. The measured temperature by LabView was then corrected by a calibration correction.

The data from the pressure sensors was acquired by means of a National Instruments NI$6250 \mathrm{PCl}$ card, at a frequency of $100 \mathrm{kHz}$. The sensors produced an output voltage of 0.5$5.5 \mathrm{~V}$, which was converted to pressure using the specified conversion constant supplied by the manufacturer.

\subsubsection{Experimental procedure for average heat flux measurement}

The experimental procedure consisted out of several steps, which were executed successively. These steps are discussed separately.

\section{Measurement initiation}

The first step in the experimental procedure was the initiation of the temperature measurement. This is done before any of the other steps to ensure that the temperature distribution in the test area is uniform. Data from the thermocouples was stored digitally for post processing. The temperature measurement was left running as further steps were taken.

Once the temperature measurement was running, the pressure sensors were also switched on. Data from the pressure sensors was not stored at this point, since this would create inconveniently large data files of data that would not be used in later stages

\section{Air supply setting}

Once the two sensor types were both running, the air supply line was set to the correct pressure. This static pressure, measured on an analog pressure indicator, was a measure for the final pressure reached in the reactor. The actual compression pressure reached in the reactor was calculated from the pressure sensor signal as a post processing step. 
Once the pressure in the supply line was set, the valve connecting the supply line to the reactor could be opened, initiating piston motion.

\section{The run}

The experiment was run for the time desired (generally in the order of half an hour, to one hour). During this time, every two minutes, starting from the moment that piston motion was initiated, the pressure measurement data was stored for a period of $1 \mathrm{~s}$. This was done so that later on a check can be made of the evenness of the pressure in the reactor, over the run time of the experiment. The temperature data of the five thermocouples was stored constantly. After the run, the reactor needed to cool down to room temperature before a new experiment could be started.

The post processing consisted of two different steps. Firstly, the data needed to be adjusted according to the calibration curves. This was done as a post processing step for the thermocouple measurements, and online for the pressure measurements. After this step, the data could be used for the various analyses done. These analysis steps will be discussed in more detail in the following section.

\subsubsection{Data analysis method}

\section{Temperature data}

The raw data, as it was acquired from the thermocouples is best depicted as shown in Figure 3.6. In this figure the temperature as it was measured by the five thermocouples is shown as a function of time, after correction by the thermocouple specific calibration curves. It is visible that all measurement points were at $291 \mathrm{~K}$ initially. As the experiment commenced, the thermocouple closest to the inside edge of the cover started heating first and at the highest rate. The thermocouples that were further away from the inside edge started heating slightly later and at a lower rate. As the experiment continued, the heating rate of all thermocouples decreased, but was not yet zero at the end of the experiment. 


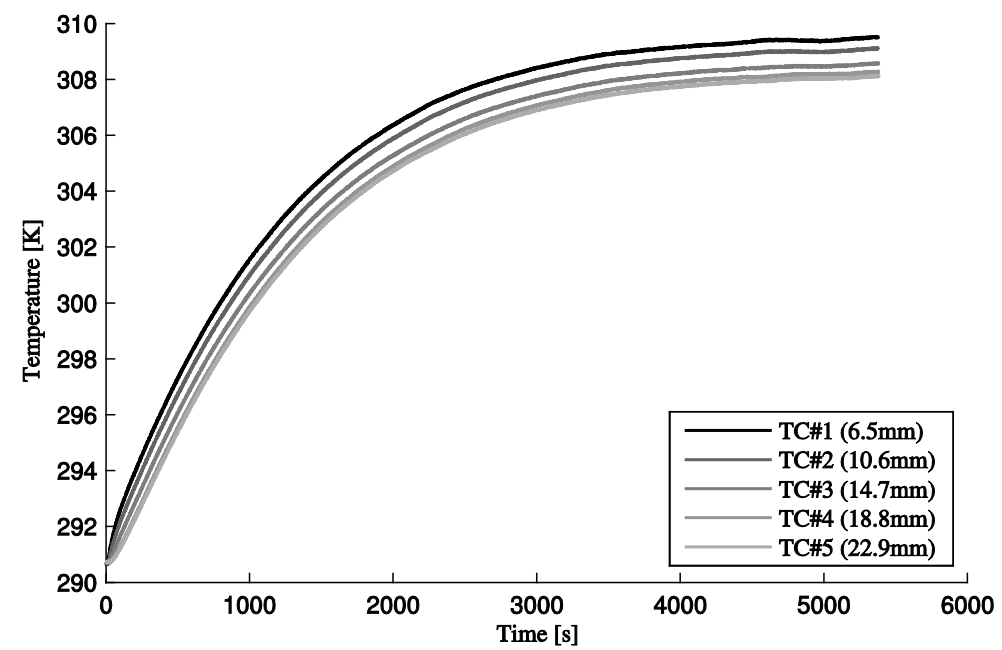

Figure 3.6, Example temperature measurement, static pressure in supply line was 5 bar

\section{Pressure data}

The pressure data required a little more processing before it was transformed into more useful data. Firstly the data needed to be shifted to remove the baseline voltage output of the sensors. Then, by using the manufacturer's calibration constants, the measured voltage could be converted to a pressure. Lastly the found pressure was shifted along the pressure axis so that a zero measured value equals one bar. This process is summarized as:

$$
p(t)=p_{0}+\frac{1000}{14.5 \cdot C_{c}}\left[U(t)-U_{0}\right] .
$$

In this equation, the constant $C_{c}$ is the calibration constant supplied by the manufacturer of the sensor in $\mathrm{mV} / \mathrm{psi}$. The factors 1000 and 14.5 convert this to volt and bar.

Finally the data, which contains quite a lot of noise, can be filtered to reduce the noise. In Figure 3.7 the result of this process is shown, for an example experiment where the pressure in the air supply line was 6.3 bar. In this figure both the filtered and unfiltered data are shown. The filter used was a Butterworth filter with a $500 \mathrm{~Hz}$ cut-off frequency. 


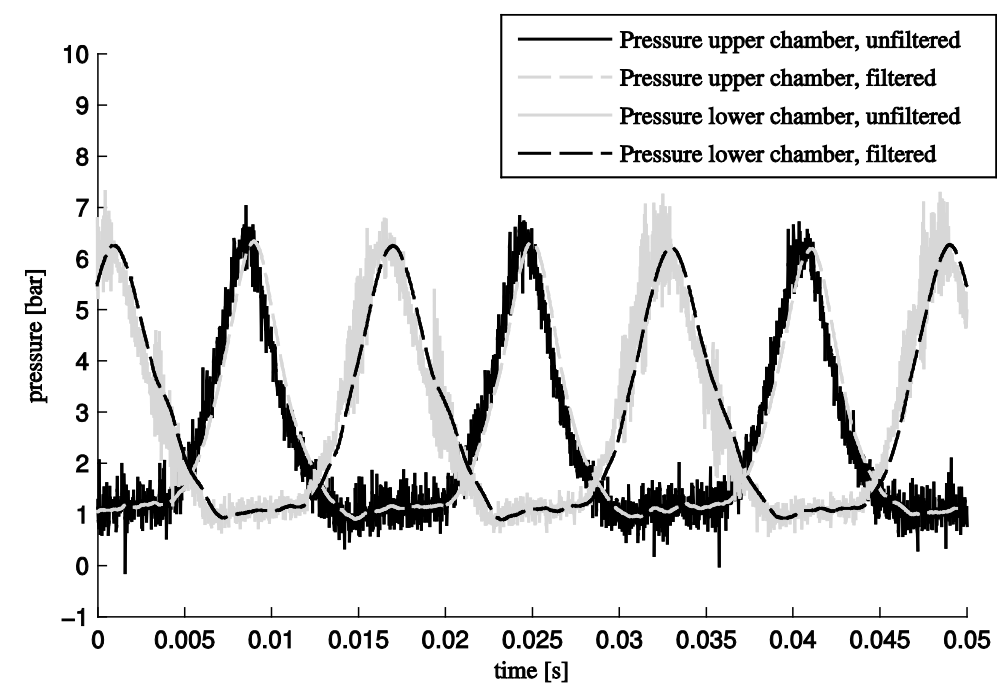

Figure 3.7, Example pressure measurement, static pressure in supply line was 6.3 bar

\section{Determining the heat flux, the average surface temperature and the heat transfer coefficient}

To calculate the average heat flux, average surface temperature and the heat transfer coefficient, the temperature data obtained can be represented in a different fashion. By plotting the data as a function of thermocouple position, at various specific times, a graph as shown in Figure 3.8 is obtained. In this graph the data from Figure 3.6 is used, and plotted at seven specific times.

To find the heat flux both through the measurement volume and through the surface of the cover, as well as the average surface temperature, different types of fits can be fitted trough the data, as it is presented in Figure 3.8. When a linear fit is used

$$
T(x)=a+b \cdot x,
$$

the average heat flux thought the measurement volume is obtained. To obtain the heat flux through the surface, and the average surface temperature, a more sophisticated fitting function is needed. To obtain this more sophisticated fitting function the analytical solution of the unsteady energy diffusion equation in a semi-infinite domain can be used. This unsteady energy diffusion equation takes the form:

$$
\frac{\partial T}{\partial t}=\alpha \frac{\partial^{2} T}{\partial x^{2}}
$$




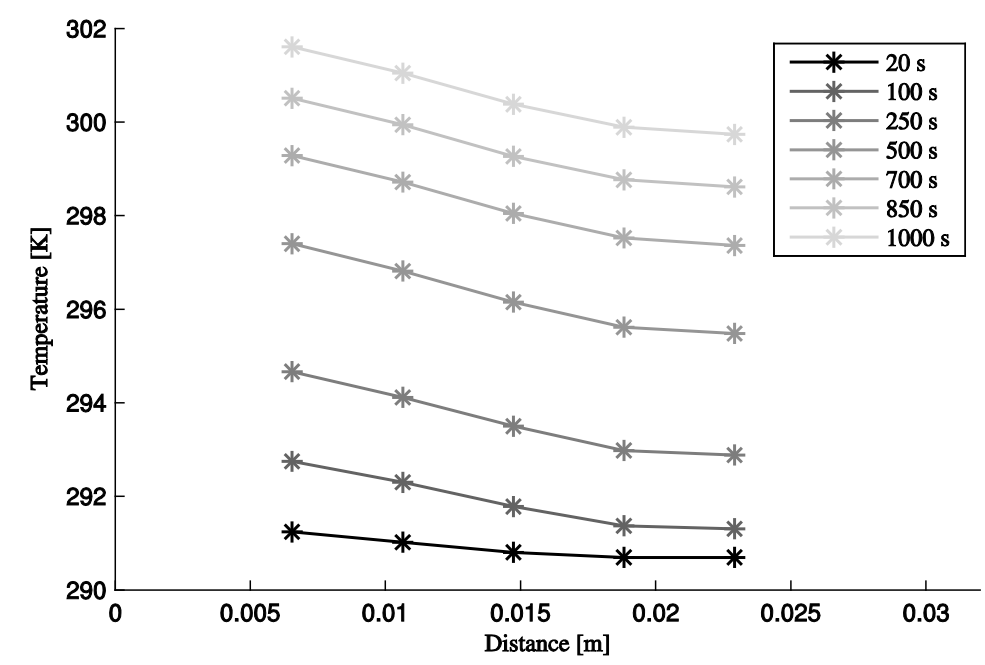

Figure 3.8, Example temperature measurement, plotted at various specific times, pressure in supply line was 5 bar

The solution of the unsteady energy diffusion equation depends on the boundary conditions. For a semi-infinite domain, and a constant wall temperature as a boundary condition, the solution takes the form of a complementary error function

$$
T(x)=T_{0}+\left(T_{w}-T_{0}\right) \operatorname{erfc}\left(\frac{x}{2 \sqrt{\alpha} t}\right) .
$$

So, even though the boundary conditions used to arrive at the solution in equation 3-6 are not exactly the same as in the experiment, it may be expected that the solution as it will be found in the experiments will have at least the same form as equation 3-6. So the more generalized version is used as a fitting function for the data

$$
T(x)=A+B \cdot \operatorname{erfc}(C \cdot x) .
$$

To reach easy convergence with the fitting software used, a close first guess of the parameters to be estimated has to be provided. If this is not done, the fitting will either not reach a solution, or take very long. The first guess used is based on the solution as it is found in equation 3-6. For parameter $A$, the value of the last thermocouple is used, for the parameter $B$, the difference between the first and last thermocouple is used, and for parameter $C$, the value of 50 is used. This last parameter value is also based on the result of equation 3-6. For the properties of the material used, the value of the denominator comes to about 5 initially, and about 200 near the end of the experiment. It was found that letting the value of $C$ vary for different fits gave worse results than simply using a 
constant value of 50, which can be explained by the fact that the solution in equation 3-6 is a solution for a semi-infinite domain, with boundary conditions that are not exactly the same as in the experiment.

The two fits that are obtained in such a way, are shown in Figure 3.9. In this figure, the data that was displayed in Figure 3.8, is shown with the two fits that are made. The linear fit intersects the $y$-axis (which is at the position of the edge of the wall), at a much lower temperature than the complementary error function fit. Also, the gradient at the intersection with the $y$-axis is lower for the linear fit than for the error function fit. At longer times the difference between the linear and complementary error function fits becomes smaller, both in gradient at the edge, as well as surface temperature.

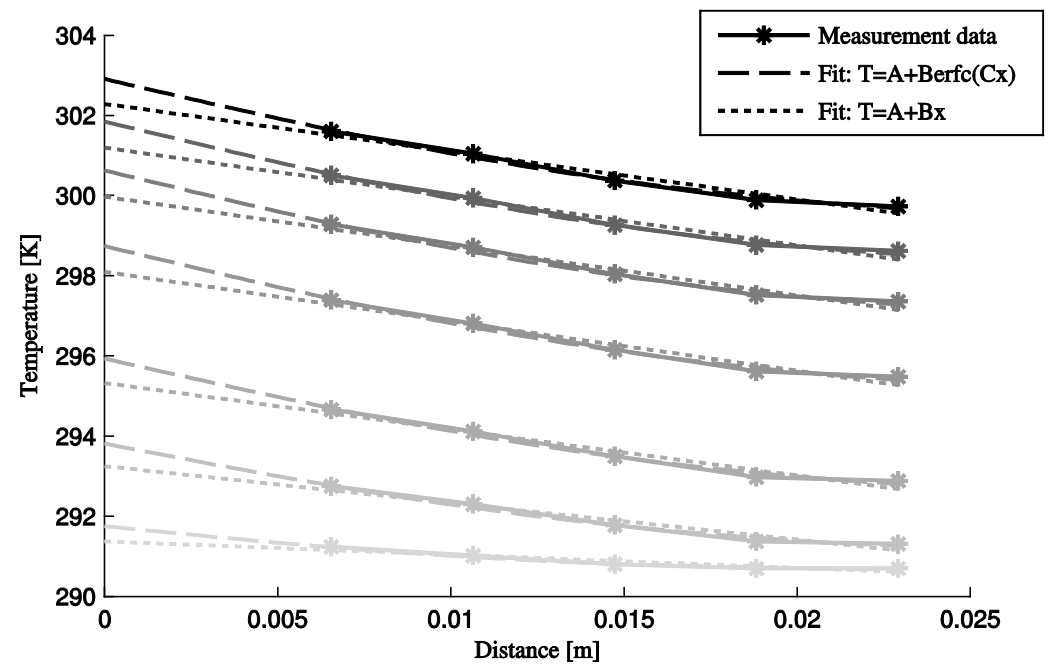

Figure 3.9, Example temperature measurement, with complementary error function fit (dashed) and linear fit (dotted), pressure in supply line was 5 bar

Once a good temperature fit is found, the average surface temperature can be found by extrapolating the fit function to $x=0$. This means that for the complementary error function fit, the surface temperature reduces to the sum of coefficients $A$ and $B$. The heat flux can also be calculated, once a good temperature fit is found: 


$$
\begin{aligned}
q(0)=-\left.k \frac{\partial T}{\partial x}\right|_{x=0}= & -\left.k \frac{\partial}{\partial x}(A+B \cdot \operatorname{erfc}(C \cdot x))\right|_{x=0}, \\
& =k \frac{2 \cdot B \cdot C}{\sqrt{\pi}} .
\end{aligned}
$$

In Figure 3.10 the result of the surface flux, as found by equation $3-8$, as well as the average flux through the measurement domain, defined as coefficient $b$ in equation 3-4 are shown. This result is obtained using $27 \mathrm{~W} \cdot \mathrm{m}^{-1} \cdot \mathrm{K}^{-1}$ as a value for the thermal conductivity of the material. Both the average and the surface heat flux are zero at the start of the experiments. This is in line with expectations. For longer times, near the end of the experiment, the surface heat flux and average heat flux through the measurement domain come more closely together, but do not seem to converge fully (though strictly the experiment would have to be longer to be sure). This is also logical, since not all heat goes through the measurement domain: some may be transferred to the surrounding air through the sides of the cover.

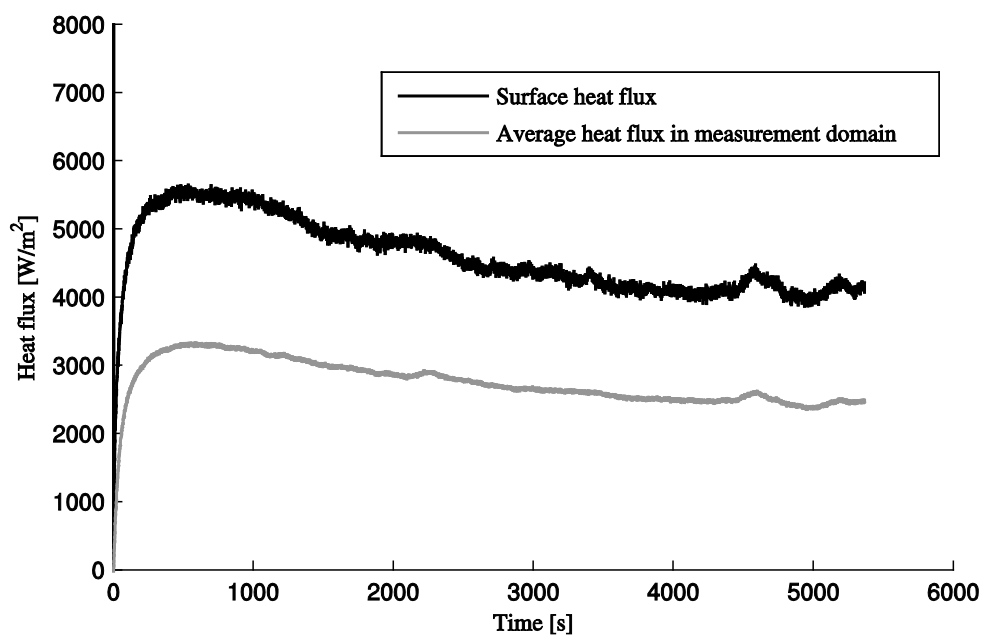

Figure 3.10, Resulting heat fluxes, the surface flux and the average heat flux through the measurement domain, static pressure in supply line was 5 bar

As a check of the validity of the results obtained, an energy balance can be made. By integrating the found surface heat flux over time the total energy, per unit of area, that enters the cover is found 


$$
E_{\text {in }}=\int_{t_{0}}^{t_{\text {end }}} q(t) d t .
$$

The total inflow of energy into the cover can be compared to the energy change of the cover:

$$
\Delta E=\int_{0}^{L} \rho \cdot C_{p} \cdot\left[T\left(x, t=t_{\text {end }}\right)-T_{0}\right] d x .
$$

Table 3.3 shows the parameters that are used, and the result this gives. The difference between the initial and final energy of the cover is about $3.1 \cdot 10^{6} \mathrm{~J} \cdot \mathrm{m}^{-2}$, which compares quite well to the total heat flux through the surface of $23.2 \cdot 10^{6} \mathrm{~J} \cdot \mathrm{m}^{-2}$, especially since the system has approached steady state, so a lot of the energy that has flown into the system will have flown out into the environment. This means energy change should be smaller, but the same order of magnitude as the heat that travelled through the surface.

\begin{tabular}{lll}
\hline \hline Parameter: & Value: & Unit: \\
\hline $\mathrm{A}(\mathrm{t}=5000)$ & 35 & $\mathrm{~K}$ \\
$\mathrm{~B}(\mathrm{t}=5000)$ & 2.68 & $\mathrm{~K}$ \\
$\mathrm{C}(\mathrm{t}=5000)$ & 48.5 & $\mathrm{~m}^{-1}$ \\
$\mathrm{~L}$ & 0.050 & $\mathrm{~m}$ \\
$\rho$ & $7.8 \cdot 10^{3}$ & $\mathrm{~kg} \cdot \mathrm{m}^{-3}$ \\
$\mathrm{C}_{\mathrm{p}}$ & 460 & $\mathrm{~W} \cdot \mathrm{kg}^{-1} \cdot \mathrm{K}^{-1}$ \\
$\mathrm{~T}_{0}$ & 293 & $\mathrm{~K}$ \\
\hline Result: & Value: & $\mathrm{Unit}:$ \\
\hline $\mathrm{E}_{\text {in }}$ & $23.2 \cdot 10^{6}$ & $\mathrm{~J} \cdot \mathrm{m}^{-2}$ \\
$\Delta \mathrm{E}$ & $3.1 \cdot 10^{6}$ & $\mathrm{~J} \cdot \mathrm{m}^{-2}$ \\
\hline \hline
\end{tabular}

Table 3.3, parameters and result of energy analysis

Finally, the acquired data can be used to calculate a heat transfer coefficient. In this case the heat transfer coefficient is defined as

$$
q(t)=h(t)\left(T_{a d-\max }-T_{w}(t)\right) .
$$

Where $h(t)$ is the time dependent heat transfer coefficient, $T_{a d-\max }$ the maximum adiabatic gas temperature and $T_{w}(t)$ the time dependent wall surface temperature. To calculate the heat transfer coefficient, the maximum adiabatic wall temperature can be calculated from the pressure signal using the ideal gas law. The time dependent wall temperature can be found by extrapolating the found fit to $x=0$. Doing this for the example measurement, a heat transfer coefficient as a function of time as shown in Figure 3.11 is found. 


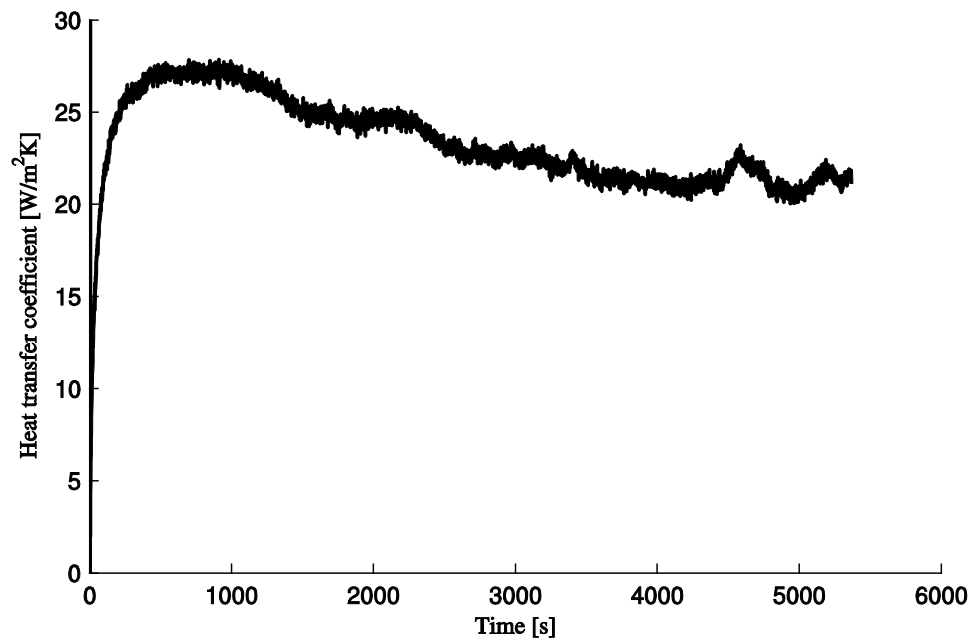

Figure 3.11, Resulting heat transfer coefficient, static pressure in supply line was 5 bar

\subsection{The single shot reactor experimental setup}

\subsubsection{Introduction}

While the average heat transfer measurements are very insightful into the general energy balance of the PCR, giving information on the steady state heat transfer from the hot reaction gas to the walls and piston, it does not provide a lot of information on the exact processes that occur within the PCR. In order to get some information on the heat flux from the compressed gas, during the compression- expansion cycle, a much faster measurement method will have to be used.

Investigation of the chemistry as it occurs in the PCR is one of the main goals of the research performed within the scope of this thesis. In order to be able to investigate exactly what happens, from a chemical point of view, during one compression expansion cycle and to be able to compare different results that vary only a single parameter, experiments must be reproducible to a great degree. This means that, among others, temperature distributions, piston motion and chemical composition of reactants must be identical for each experiment. One way to obtain this reproducibility is by using a single shot reactor. This completely eliminates the effect of previous compression expansion cycles, with the associated effects of heat release, products remaining in the reactor and piston velocity fluctuations. 
So, the goal of the single shot reactor setup is the following:

1. To design and build a single shot reactor, that can mimic a single compression expansion cycle of the PCR, with good control over process parameters

2. To develop an experimental procedure to do experimental parameter studies towards the chemistry of syngas generation from methane

3. To perform the parameter study, isolating the following parameters:

- Reactant composition

- Initial reactor and reactant temperature

- Piston kinetic energy

- Piston mass

- Cover geometry

4. To develop an accurate and robust method for measuring the instantaneous heat flux from the PCR, within one cycle, for different operating conditions

5. To test this method by measuring the instantaneous heat flux at various operating conditions.

6. To arrive at an empirical relation between pressure and heat flux.

In the following sections firstly the setup is addressed: the various parts that it consists of and its working. Then the different sensors are explained, also paying attention to calibration (if necessary). Lastly the different data analysis procedures, for the various data streams generated by the setup are treated. 
(15)

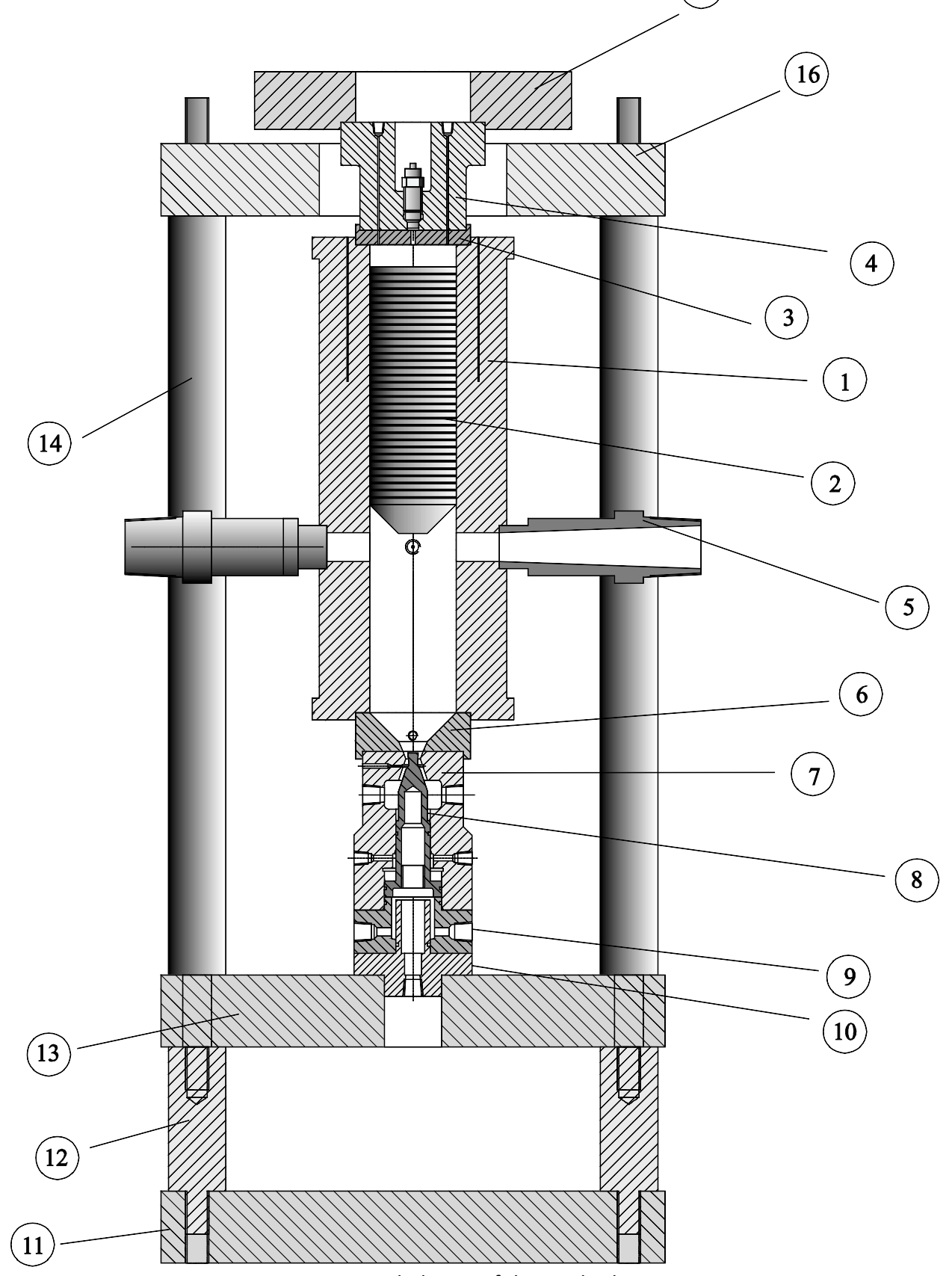

Figure 3.12, Detailed view of the single shot reactor 


\subsubsection{Experimental Setup}

In order to be able to perform the experiments that were outlined in the goals, full control is needed over the initial reactor temperature and the piston motion. To achieve this controllability, a single shot reactor was designed by Energy Conversion Technologies BV and used for the single shot experimental setup. In this reactor one compression-expansion cycle of the continuous process is replicated. This ensures that important process parameters such as reactor and piston temperature are the same for each experiment. It also allows for very good control of the initial mixture in the reaction chamber, by avoiding the influence of products remaining in the reactor from previous cycles (though heat flux measurements were done without chemical reactions). A detailed, partly cross sectioned, view of the single shot reactor is shown in Figure 3.12. In this figure the different parts are numbered. The numbered parts are listed in Table 3.4.

\begin{tabular}{ccc}
\hline \hline Part number & Part name & Construction material \\
\hline 1 & Cylinder & Corrax [49]* \\
2 & Piston & Poco ACF-10Q / Toyo Tanso GM-5 \\
3 & Top cover & SS 304 \\
4 & Top sensor housing & SS 304 \\
5 & Exhaust pipe (2) & SS 304 \\
6 & Lower cover & SS 304 \\
7 & High speed valve housing & Corrax* \\
8 & Valve shoe & Corrax* \\
9 & Lower valve spacer & SS 304 \\
10 & Lower valve cover & SS 304 \\
11 & Auxiliary support plate & Carbon steel, zinc plated \\
12 & Auxiliary support column (4) & Carbon steel, zinc plated \\
13 & Lower support plate & Carbon steel, zinc plated \\
14 & Main support column (4) & Carbon steel, zinc plated \\
15 & Compression plate & Carbon steel, zinc plated \\
16 & Upper support plate & Carbon steel, zinc plated \\
& *Heat treated to a hardness of 48-50 HRC \\
\hline \hline
\end{tabular}

Table 3.4, Part numbers and names of the single shot reactor

\begin{tabular}{cc}
\hline \hline Bore diameter & $60 \mathrm{~mm}$ \\
Bore length & $325 \mathrm{~mm}$ \\
Piston length & $185 \mathrm{~mm}$ \\
Piston mass & $0.854 \mathrm{~kg} / 3.16 \mathrm{~kg}$ \\
\hline \hline
\end{tabular}

Table 3.5, Characteristic sizes and weights of the single shot reactor 
For stability the entire construction is bolted to a concrete block of approximately $500 \mathrm{~kg}$. This block is in turn mounted on shock absorbing rubber mounts. Some characteristic sizes and the piston masses are shown in Table 3.5.

The setup is fitted with four different types of sensors: a fast surface temperature sensor, dynamic pressure sensors, a piston velocity sensor and several "slow" thermocouples.

\section{Reactor heating}

In order to be able to do experiments at elevated initial temperature, the reactor is equipped with a heating system. This is comprised of two separate heating wires, a $1250 \mathrm{~W}$ heating wire that is wrapped around the cylinder and a $250 \mathrm{~W}$ heating wire that is wrapped around the top sensor housing. Thermocouples built into the body of the cylinder and into the body of the top cover monitor if the desired preheat temperature is reached. To avoid overheating of the heaters, stand alone thermocouples (not integrated into the data acquisition system) are used for the control of the heaters. These are directly connected to thermostatic units and mounted directly next to the heating wires. To reduce losses, the entire cylinder is surrounded by a layer of insulating glass wool and the top sensor housing is wrapped in a layer of ceramic paper. To avoid overheating of the air hoses attached to the launch mechanism (which have a maximum admissible temperature of $373 \mathrm{~K}$ ), a thermocouple is taped to one of the hoses that leads to the upper ports in the launch mechanism. The data acquisition system is configured in such a way that the thermostatic units only receive power if the hose temperature is below $333 \mathrm{~K}$.

\section{The launch mechanism}

Movement of the piston is initiated by injection of compressed air through the launch mechanism. As the piston moves up, at some point it (if enough energy is supplied) unblocks the outlet ports on the side of the reactor. This allows part of the launch air to escape. When the piston moves down again under the force of the now compressed gasses above the piston as well as gravity (the latter of which is generally much smaller), the motion of the piston is damped by a small hole in the lower cover. This works against the buildup of pressure under the piston, ensuring that the second reciprocation of the piston is significantly damped.

The launch mechanism is connected to a control board that supplies the compressed air. A schematic view of the control board is shown in Figure 3.14. In Figure 3.15 the symbols that are used are explained. 
In Figure 3.13 the different chambers in the launch mechanism are illustrated. To use the launch mechanism, the following connections are made:

- Connections $6 \mathrm{~A}$ and $6 \mathrm{~B}$ are connected by flexible hose to the ports in the lower valve spacer (counter pressure chamber)

- Connections 5A and 5B are connected by flexible hose to the upper ports in the launch mechanism (launch chamber)

- Connections $4 \mathrm{~A}$ and $4 \mathrm{~B}$ are connected by flexible hose to the middle ports in the launch mechanism (trigger chamber)

- Connection 2A is connected by capillary tube to an opening in the lower cover

- Connection 8A is connected by capillary tube to one of the two openings in the top sensor housing

- Connection 8B is connected to the bottle of reactants

- Connection $8 \mathrm{C}$ is connected to a bottle of compressed nitrogen

- Connection $1 \mathrm{~A}$ is connected flexible hose to the air compressor

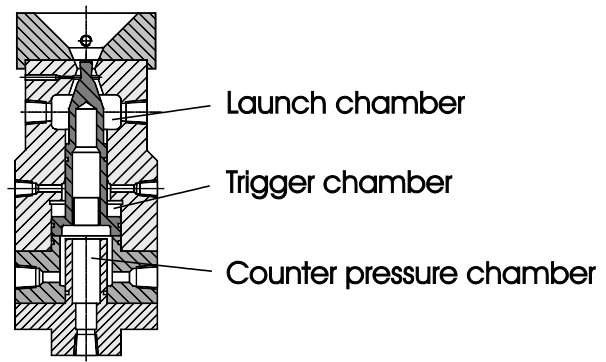

Figure 3.13, Different chambers in the launch mechanism

The launch procedure is, with connections made as described, stepwise as follows.

1. All valves are closed. Valve 101 and reducer 103 are used to pressurize the system to sufficient pressure, for example, 80 bar.

2. Needle valve 601 is used to pressurize ports $6 \mathrm{~A}$ and $6 \mathrm{~B}$ to a predefined counter pressure, for example 30 bar. This pressure pushes the valve shoe in the launch mechanism up, closing the gap between the conical top of the valve shoe and the high speed valve housing thus closing the launch chamber.

3. Valve 501 is used to pre-pressurize the system up to valve 504 , to a predefined launch pressure, for example 80 bar.

4. Valves 506 and 507 are opened, to permit air flow to the launch chamber in the launch mechanism from the control board when valve 504 is opened. 
5. Valve 401 is used to pre-pressurize the system up to valve 404 , to a predefined trigger pressure, for example 80 bar.

6. Valves 405, 406, 407 and 409 are opened, to permit the air flow from the system to trigger chamber in the launch mechanism when valve 404 is opened to escape to the environment.

7. After opening valve 504 electronically, the system is allowed to stabilize for about 10 seconds for the pressure to equalize. After this, valves 504, 506 and 507 are closed. The launch chamber in the launch mechanism is now charged with 80 bar. However, the pressure of 30 bar that was set as a counter pressure keeps the valve closed, because the pressure of 30 bar works on a larger area than the 80 bar launch pressure.

8. To launch the piston, valve 404 is opened for $10 \mathrm{~ms}$ electronically. This allows some of the pressurized gas stored in receiver $\mathrm{R} 4$ to flow through connections $4 \mathrm{~A}$ and $4 \mathrm{~B}$. The hydraulic resistance beyond connections $4 \mathrm{~A}$ and $4 \mathrm{~B}$ (three valves and a zero return valve) make that the pressure in the trigger chamber briefly climbs. The combined pressure of the preset launch pressure, and the briefly applied trigger pressure push the valve shoe down, briefly opening the gap between the valve shoe and high speed valve housing. This allows some of the launch gas to flow into the bottom of the reactor, initiating the piston motion. The result is that the pressure in the launch chamber drops, while simultaneously the trigger pressure also escapes through valve 405 and 407 . The counter pressure on connections $6 \mathrm{~A}$ and $6 \mathrm{~B}$ is now high enough to close the valve again. The design of the launch mechanism is such that the motion of the valve shoe is faster than the reciprocation of the piston, so the valve is closed when the piston returns down.

9. To determine the different pressures needed, the following relations were used:

$$
\begin{gathered}
P_{\text {trigger }}=P_{\text {launch }} \\
P_{\text {counter }}=\max \left(\frac{P_{\text {launch }}}{2}-10,10\right) .
\end{gathered}
$$




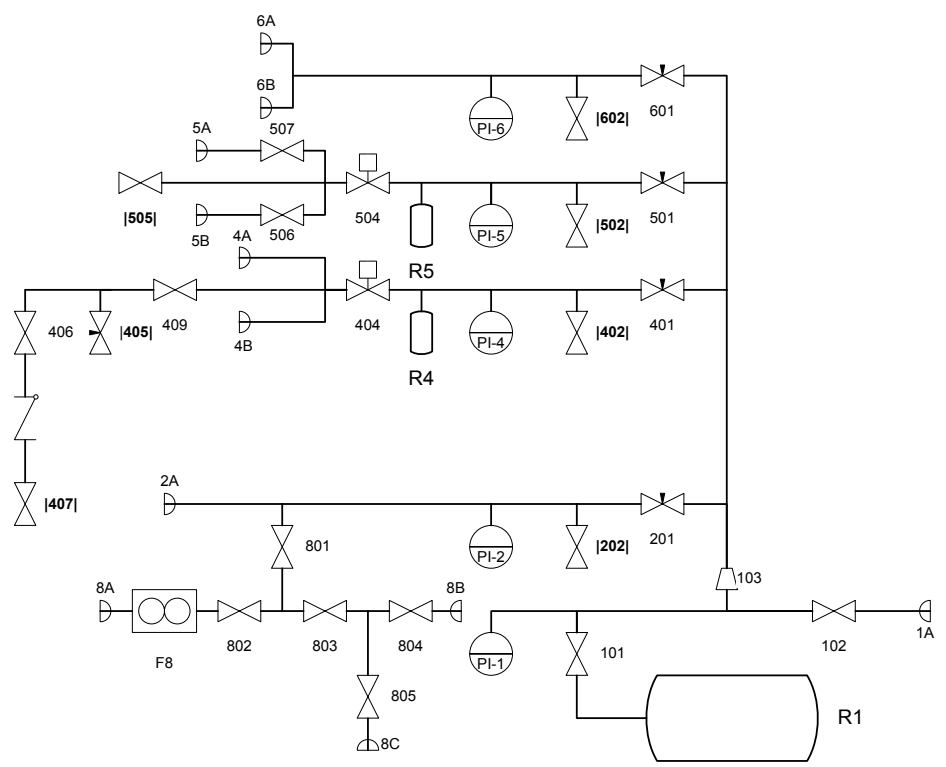

Figure 3.14, Schematic view of the control board

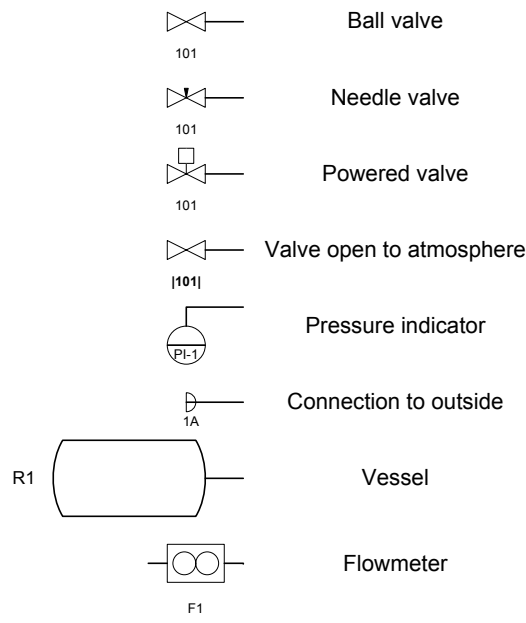

Figure 3.15, Symbols used in Figure 3.14

\section{Characteristic process parameters reached}

The single shot reactor is capable of reaching high pressure and temperature combinations that cannot be reached in conventional reactors. In Table 3.6 the order of magnitude of characteristic process parameters reached are shown. 


\begin{tabular}{ccc}
\hline \hline Parameter & \multicolumn{2}{c}{ Order of magnitude } \\
\hline P max & $0.854 \mathrm{~kg}$ piston & $3.16 \mathrm{~kg}$ piston \\
T max & $\pm 700 \mathrm{bar}$ & $>1000 \mathrm{bar}$ \\
V piston max & $>2000 \mathrm{~K}$ & $>2000 \mathrm{~K}$ \\
Compression ratio max & $\pm 30 \mathrm{~m} \cdot \mathrm{s}^{-1}$ & $\pm 17 \mathrm{~m} \cdot \mathrm{s}^{-1}$ \\
\hline \hline
\end{tabular}

Table 3.6, Characteristic process parameters reached

\section{Partial oxidation reactant injection}

The reactants needed to investigate the partial oxidation process are injected through the capillary tube (playing the role of a check valve during compression) shown in the top sensor housing and the top cover. These reactants are kept premixed in a 10 I vessel, under an initial pressure of 150 bar (as more experiments are done this pressure slowly decreases). This pressure is reduced to 5 bar before reaching valve 804 . Before any experiment, the reactor is flushed with a flow of $1.1 \cdot 10^{-5} \mathrm{~m}^{3} \mathrm{~s}^{-1}$ of nitrogen through connection $8 \mathrm{C}$, for 5 minutes (this corresponds to a total volume injected of approximately five times the reactor volume). After this, valve 805 is closed and the reactor is flushed for 3 minutes with reactants by opening valve 804 . After the flushing with reactants the piston is launched and products are retrieved.

\section{Steam injection}

Investigation of the chemical process of steam reforming is experimentally slightly more complicated than the partial oxidation. The reason is that the mixture of methane-steam cannot be premixed into a vessel, since the steam would condense at room temperature (so the vessel would have to be stored at elevated temperature, adding many complications). Instead, the steam and a methane-argon mixture are mixed at the moment of injection into the reactor. This is done using a modification of the setup, shown in Figure 3.16. In this figure a syringe is shown, which is connected to a side branch in the capillary tube that leads from connection $8 \mathrm{~A}$ to the reactor. The injection of the steam-methane-argon mixture is done according to the following procedure.

1. Fill the syringe with demineralized water, the volume of water in the syringe must be at least $40 \mu \mathrm{l}$ more than the amount that is intended to be injected

2. Connect the syringe to the capillary tube

3. Flush the reactor with nitrogen at a flow rate of $1.1 \cdot 10^{-5} \mathrm{~m}^{3} \mathrm{~s}^{-1}$, to remove any residual moisture for 5 minutes.

4. Follow points 1-6 of the launch procedure, to prepare piston for launch

5. Flush the reactor with the $\mathrm{CH} 4-\mathrm{Ar}$ mixture, for 1.5 minutes

6. Inject water until the correct amount is left in the syringe, while still flushing 
7. Flush for another 1.5 minutes

8. Close valve 802 , make pressure in connector hose to bottle $2.5 \mathrm{bar}$, then close valve 804

9. Inject water into the capillary tube

10. Open valve 802 , the pressurized reactants in the tube between valve 802 and 804 inject the water

11. Allow the reactor to stabilize for one minute, while performing steps 7 and 8 of the launch procedure

12. Retrieve products

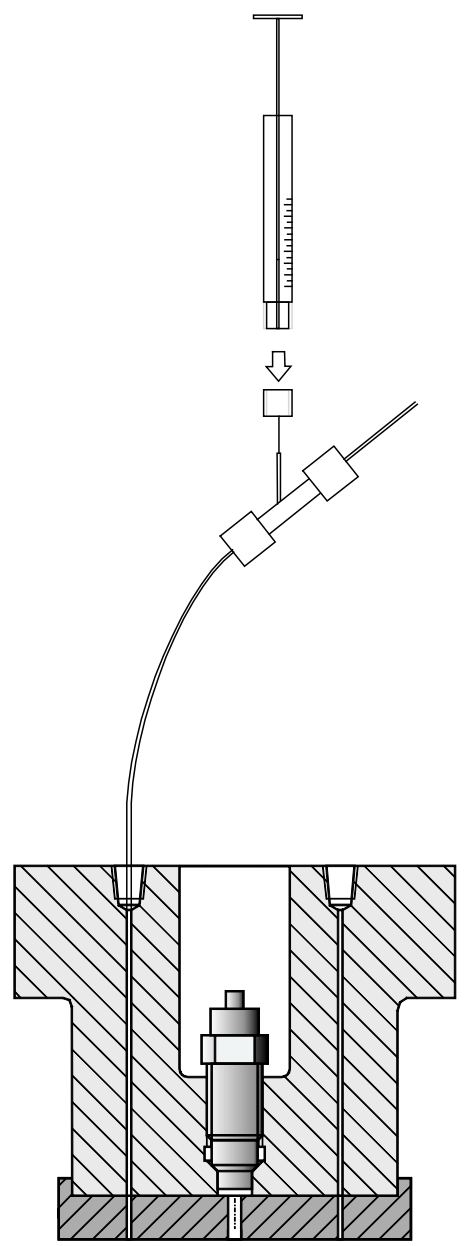

Figure 3.16, Schematic view of the steam injection mechanism 
This procedure ensures that any dead volumes within the water injection system are filled with water prior to injection and that indeed the amount of water that is intended to be injected reaches the reactor. The volume between valves 802 and 804 is large enough that all the water injected into the capillary is carried into the reactor, but small enough to prevent it from being blown straight through.

\section{Piston mass}

The mass of the piston is expected to have a significant effect on the chemistry that occurs in the PCR. For this reason, results obtained with pistons of different mass are compared. In Table 3.4 the piston material is shown to be two types of materials. The first piston, the "light" piston is used for the bulk of the experiments and constructed from Poco ACF-10Q [50] graphite. The other piston, the "heavy" piston is used for comparison with the results obtained with the light piston and constructed from a more dense graphite, the metal bonded graphite Toyo Tanso GM-5 [51]. The density of the

light piston material is $1.77 \cdot 10^{3} \mathrm{kgm}^{-3}$ giving the light piston a mass of $0.86 \mathrm{~kg}$. The density of the heavy piston material is $6.20 \cdot 10^{3} \mathrm{kgm}^{-3}$ giving the heavy piston a mass of $3.16 \mathrm{~kg}$, approximately 3.7 times that of the light piston.

\section{Cover geometry}

To investigate the flame quenching effect that the cover may have on the chemistry that occurs in the reactor, different cover geometries are tested. The goal of the new geometries is to increase the size of the adiabatic core within the reactor, thus reducing the amount of reactants that are affected by the presence of the walls. The alternative cover designs that have been tested are shown in Figure 3.17. The designs are such that the reactor volume that is added by the cavities in the alternative cover designs is compensated for by allowing the covers to protrude slightly into the reactor cylinder, thus keeping the total reactor volume equal. 

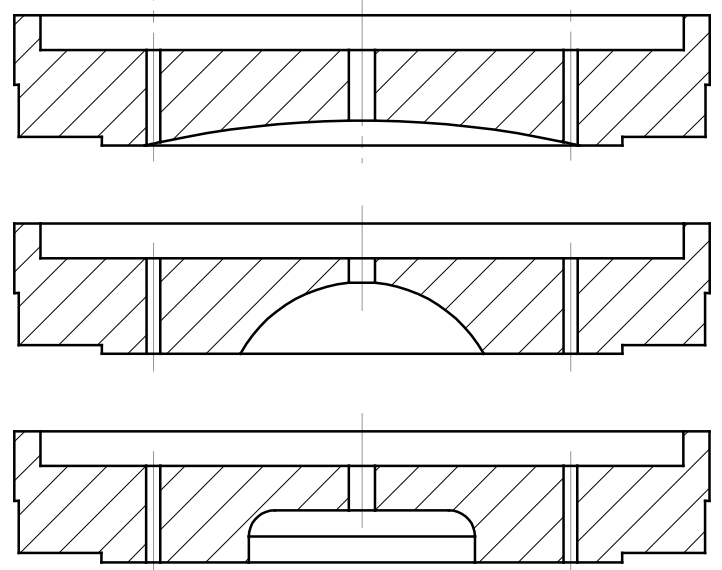

Figure 3.17, Design of the alternative covers tested. Cover 1 (top), cover 2 (middle) and cover 3 (bottom)

\subsubsection{Sensors}

Three different sensors are used to monitor important parameters during the experiment. A fast surface thermocouple is used to measure the surface temperature. High speed pressure sensors monitor the pressure in the reactor (Optrand D732A8, D532A8 and D53294) for the purpose of viewing the pressure history of the gas in the reactor, and the pressures in the different chambers of the launch mechanism. A third sensor type is used to determine the velocity of the piston. This is done by a fiber optic sensor (Keyence FS-V31M), which shines a source of modulated light through a fiber optic wire onto the side of the piston. As the piston moves, a different amount of light is reflected depending on whether the light hits the flat surface of the piston or a passing groove. From the changing signal in reflected light the moment of passing grooves can be determined and from this the velocity of the piston. All three types of sensors used: temperature, pressure and velocity, will be discussed in more detail in the following sections.

\section{Fast surface temperature sensors}

The best way to get a good measurement of the instantaneous heat flux from the hot compressed gas is by measuring the surface temperature of the adjacent wall, fast enough to obtain its change during one cycle. This surface temperature can then be postprocessed into a heat flux analytically.

There are several temperature measuring sensors or methods to be found in literature that are reported to have a time resolution (or response time), capable of following the 
rapid temperature changes as they occur in the PCR. Of these, the following have been considered to be used for the purposes of instantaneous heat flux measurement:

- Thin film thermocouples

- Thin film thermistors

- Thermo reflectance bolometry

- $\quad$ Eroding thermocouples

The main characteristic that all these sensors and methods have in common is that the designs strive to a sensor that is as thin as possible. The reason is that the thinner the sensor, the closer the measured temperature will be to the actual surface temperature. The response time of all of the sensors that were considered be discussed depends on the thickness of the sensing surface that needs to heat up. As shown before the time needed for heat to penetrate a certain distance can be estimated by the Fourier number, as given in equation 3-1.

If the time scale of one complete PCR cycle is assumed to be $1 \cdot 10^{-1} \mathrm{~s}$, it is not unreasonable to demand that the response time of the sensor used for measuring the surface temperature is at least 1000 times faster. The reason is that the really high temperatures and pressures as they occur in the PCR, occur during only a fraction of the entire cycle.

To investigate the instantaneous heat transfer in the PCR, any of the four mentioned measurement methods or sensors could be used. In appendix I the characteristics and working of the first three are discussed. All have a fast enough response time, large enough measurement range and may be constructed in such a way that they would withstand the high pressure in the PCR. However, from a point of view of robustness, the eroding thermocouple has a distinct advantage over the others. While the junction of the thermocouple may be more fragile than the thin films of the other sensors, any damage to the junctions can simply be restored. Additionally, the manufacturing costs of the eroding thermocouple are also quite significantly lower than that of thin film based techniques, since no expensive metal deposition machines are required to create thin films. Based on these arguments, the eroding thermocouple was employed to investigate the instantaneous heat transfer in the PCR. The working of the eroding thermocouple is as follows. 


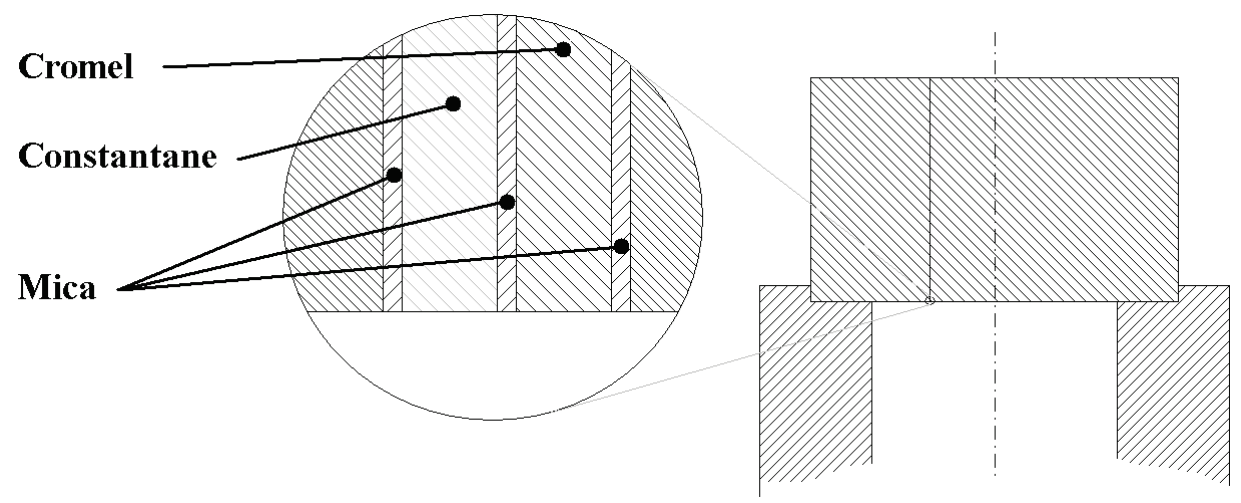

Figure 3.18, Schematic view of the eroding thermocouple

The technique of the eroding thermocouple was used before by Oude Nijeweme [52], Xiaowei Wang [53] and Gatowski et al. [54]. The thermocouple consists of a two ribbons of different materials, sandwiched between two sheets of mica and separated by a third sheet. This sandwich is embedded in the bulk material, running vertically through the material and protruding on both sides. This is schematically shown in Figure 3.18. Before use, the inner surface of the thermocouple is grinded with fine sanding paper. This forms micro junctions across the mica between the two metal sheets, creating a thermocouple circuit. The temperature of the micro junctions can now be measured by measuring the potential difference between the two protruding sheets on the outside of the reactor.

The response time of the eroding thermocouple depends on the size of the micro junctions formed. When the thickness of the metal and mica sheets are in the order of microns, for example metal sheets of $25 \mu \mathrm{m}$ and mica sheets of $5 \mu \mathrm{m}$ (which are the sizes used in this surface thermocouple), the size of the micro junctions will be in order of nanometers. This makes the expected response time of the eroding thermocouple significantly faster than thin film based sensors.

Another advantage of the sensor is its reusability. While the micro junctions are very fragile, and may break when small stresses are applied, they can be restored easily by re-grinding the surface area (hence the term "eroding thermocouple"). The manufacturing costs of the eroding thermocouple are also quite significantly lower than that of thin film based techniques, since no expensive metal deposition machines are required to create thin films.

The specific eroding thermocouple that was custom made for the experiments within this thesis was manufactured in the form of a replaceable plug by NANMAC Corporation. The plug design is shown in Figure 3.19. In this design a reference thermocouple, $0.5 \mathrm{~mm}$ under 
the surface is also visible. The two thermocouple materials used were constantan and chromel, making the sensor an E-type thermocouple.

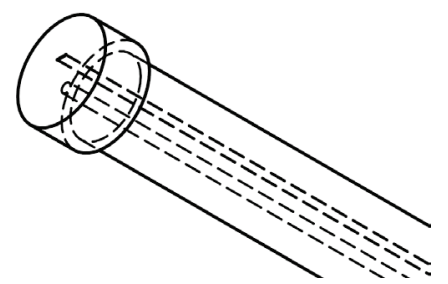

Figure 3.19, Design of the eroding thermocouple plug

\section{Pressure sensors}

Different high speed dynamic pressure sensors are used to monitor the pressure in the reactor (Optrand D732A8, D532A8 and D53294) for the purpose of viewing the pressure history of the gas in the reactor, and the pressures in the different chambers of the launch mechanism. Some characteristics of theses sensors, as supplied by the manufacturer are shown in Table 3.7.

\begin{tabular}{cccc}
\hline \hline $\begin{array}{c}\text { Sensor } \\
\text { model }\end{array}$ & $\begin{array}{c}\text { Pressure } \\
\text { rating }\end{array}$ & Size & Bandwidth \\
\hline D732A8 & 0-700 bar & $1 / 2-20$ UNF & $1.0 \mathrm{~Hz}-30 \mathrm{kHz}$ \\
D53294 & $0-340$ bar & M5x0.5 & $1.0 \mathrm{~Hz}-30 \mathrm{kHz}$ \\
D532A8 & $0-340$ bar & $1 / 2-20$ UNF & $1.0 \mathrm{~Hz}-30 \mathrm{kHz}$ \\
\hline \hline
\end{tabular}

Table 3.7, Characteristics of the used pressure sensors

While the pressure sensors are supplied with a calibration constant relating the output voltage to the pressure the sensor is exposed to, a calibration was necessary. To calibrate the pressure sensors a special calibration hub was made, shown in Figure 3.20. In this hub, two large pressure sensors could be mounted (for use in the top sensor housing) and three small pressure sensors could be mounted (for use in the lower cover, high speed valve housing and lower valve spacer). 


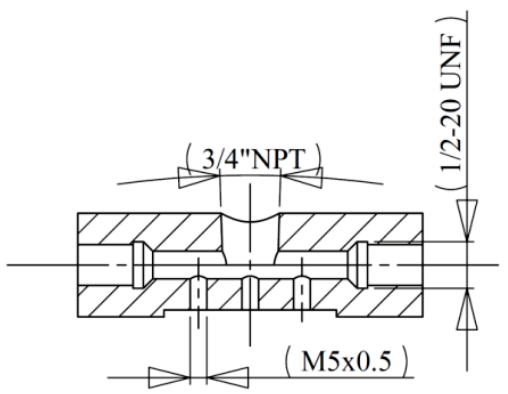

Figure 3.20, The pressure sensor calibration hub

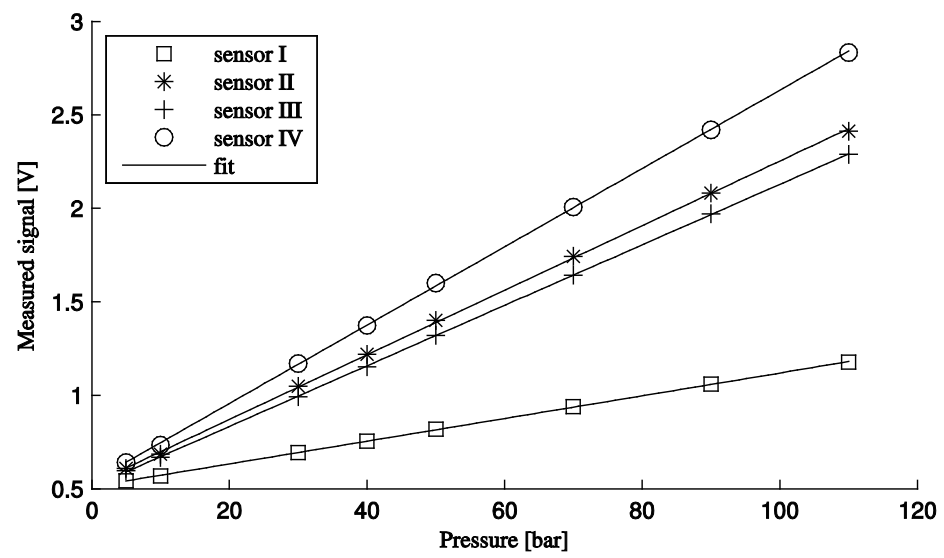

Figure 3.21, Calibration curves for the pressure sensors

The calibration hub was temporarily connected to the port $5 \mathrm{~A}$ of the control board, with port 5B capped. Valves 101 and 501 were opened, and reducer 103 used to prepressurize the system to the desired pressure. Then valve 504 was electronically opened and the sensor signals digitally stored for $1 \mathrm{~s}$ at a rate of $1 \mathrm{kHz}$. The pressure in the hub rose from ambient to the preset pressure of the control board almost instantly. Pressures between 5 bar and 110 bar were applied. The experiment was repeated for each set pressure nine times. After completion of the entire series, the locations of the pressure sensors in the hub were switched, to avoid influence of the location on the signal. The entire procedure was then repeated.

Once all the data had been obtained, linear fits were used on the averaged, filtered signals. The fit takes the form of

$$
P=A \cdot(U-B)
$$


where $\mathrm{U}$ is the measured voltage, $\mathrm{B}$ is the static signal at 1 bar and $\mathrm{A}$ can be compared to the calibration constant as supplied by the manufacturer. The resulting data points (averaged over the nine measurements) and the obtained fits are shown in Figure 3.21.

\begin{tabular}{cccccc}
\hline \hline Sensor & Type & $\begin{array}{c}\text { Manufacturer } \\
\text { supplied calibration } \\
\text { constant }\left[\text { bar } \cdot \mathrm{V}^{-1}\right]\end{array}$ & $\begin{array}{c}\text { Experimentally } \\
\text { determined } \\
\text { constant A } \\
{\left[\text { bar } \cdot \mathrm{V}^{-1}\right]}\end{array}$ & $\begin{array}{c}\text { Experimentally } \\
\text { determined } \\
\text { constant B [V] }\end{array}$ & $\begin{array}{c}\text { Standard } \\
\text { deviation after } \\
\text { calibration } \\
\text { [bar] }\end{array}$ \\
\hline I & D732A8 & 157 & 164.7 & 0.512 & 0.76 \\
II & D53294 & 61.0 & 57.88 & 0.525 & 0.37 \\
III & D53294 & 60.0 & 47.74 & 0.537 & 0.58 \\
IV & D532A8 & 61.0 & 61.77 & 0.510 & 0.37 \\
\hline \hline
\end{tabular}

Table 3.8, Manufacturer supplied and experimentally determined pressure sensor calibration constants

The calibration result for each of the sensors is shown in Table 3.8. In this table the average standard deviation, between measurements of the same pressure is also shown. This is an indication for the accuracy with which the pressure can be measured. From this standard deviation it can be concluded that the accuracy with which sensor I can measure is approximately \pm 1.5 bar ( 2 standard deviations), and for the other three sensors about \pm 1 bar. This is more than enough for the experiments performed within this project.

\section{Velocity sensor}

The velocity of the piston is measured by a fiber optic sensor (Keyence FS-V31M), which shines a source of modulated light through a fiber optic wire onto the side of the piston. As the piston moves, a different amount of light is reflected depending on whether the light hits the flat surface of the piston or a passing groove. From the changing signal in reflected light the moments of passing grooves can be determined and from this the kinetic energy of the piston.

\subsubsection{Data acquisition method}

To digitally acquire and store the measurement signals, as well as electronically control the electronic valves, two data acquisition cards are used. Both are National Instruments $\mathrm{PCl}$ cards. Some important specifications are shown in Table 3.9. 


\begin{tabular}{ccc}
\hline \hline Parameter & NI-6250 & NI-6280 \\
\hline ADC resolution & 16 bits & 18 bits \\
Number of channels & 8 differential or 16 single & 8 differential or 16 single \\
& ended & ended \\
Maximum sampling rate & $1.25 \mathrm{MS} \cdot \mathrm{s}^{-1}$ single channel & $625 \mathrm{kS} \cdot \mathrm{s}^{-1}$ single channel \\
& $1.00 \mathrm{MS} \cdot \mathrm{s}^{-1}$ multi channel & $500 \mathrm{kS} \cdot \mathrm{s}^{-1}$ multi channel \\
& (aggregate) & (aggregate) \\
Input range & $\pm 10 \mathrm{~V}, \pm 5 \mathrm{~V}, \pm 2 \mathrm{~V}, \pm 1 \mathrm{~V}$, & $\pm 10 \mathrm{~V}, \pm 5 \mathrm{~V}, \pm 2 \mathrm{~V}, \pm 1 \mathrm{~V}$, \\
& $\pm 0.5 \mathrm{~V}, \pm 0.2 \mathrm{~V}, \pm 0.1 \mathrm{~V}$ & $\pm 0.5 \mathrm{~V}, \pm 0.2 \mathrm{~V}, \pm 0.1 \mathrm{~V}$ \\
\hline \hline
\end{tabular}

Table 3.9, Specifications of data acquisition cards

As can be seen from Table 3.9, the two cards differ on two important points: ADC resolution and sampling rate. The $\mathrm{NI}-6250$ is faster, but less accurate, the $\mathrm{NI}-6280$ is more accurate, but less fast. The $\mathrm{NI}-6250$ is used in the $\pm 5 \mathrm{~V}$ mode to sample the pressure sensors (four in total) and the optical sensor. The NI-6280 is used in $\pm 0.1 \mathrm{~V}$ mode, to sample the two thermocouples (the eroding surface thermocouples and the reference thermocouple built in at $0.5 \mathrm{~mm}$ depth into the eroding thermocouple probe). The two cards are connected by RTSI cable to synchronize the sampling. Sampling is done at a maximum rate of $200 \mathrm{kS} / \mathrm{s}$. All sensors are connected differential, to reduce measurement noise.

\subsubsection{Instantaneous heat flux data analysis method}

As mentioned, the measured surface temperature profile can be used to calculate the heat flux from the hot compressed gas. The data obtained by the experiment contains some degree of electronic noise. In order to make the data more suitable for data analysis, the data is filtered by means of a fifth order Butterworth low-pass filter, with a cut off frequency equal to one twentieth of the sample frequency (for most experiments the sample frequency was $200 \mathrm{kHz}$ ). This reduces the amplitude of the noise in the measurements significantly enough to make further analysis of the results possible and meaningful. In order to show the effect of filtering, in the following paragraphs at several occasions the filtered and unfiltered data are compared.

To analyse the data obtained, and find the heat flux from the compressed gas into the reactor cover, the solution to the penetration problem into a semi infinite domain with a non constant surface temperature, taken from Hoogendoorn et al. [55], was applied. This solution

$$
T(x, t)=\int_{0}^{t} \frac{d T_{0}}{d t^{\prime}} \operatorname{erfc}\left(\frac{x}{\sqrt{4 \alpha\left(t-t^{\prime}\right)}}\right) d t^{\prime},
$$


is a function of the time derivative of the wall surface temperature $T_{0}$. The heat flux is then:

$$
q(t)=-\left.k \frac{\partial T}{\partial x}\right|_{x=0}=-\left.\frac{k}{\sqrt{\alpha}} \int_{0}^{t} \frac{d T_{0}}{d t^{\prime}} \frac{\partial}{\partial x} \operatorname{erfc}\left(\frac{x}{\sqrt{4 \alpha\left(t-t^{\prime}\right)}}\right)\right|_{x=0} d t^{\prime} .
$$

The derivative of the surface temperature with respect to time that occurs in the equation should somehow be extracted from the measurement data. To apply this equation to the measurement data obtained, the integral in equation 3-15 is written as a summation of discrete integrals, each over the length of one measurement time step:

$$
q(t)=-\frac{k}{\sqrt{\pi \alpha}} \sum_{i=1}^{N-1} \int_{t_{i}}^{t_{i}+1} \frac{d T_{0}}{d t^{\prime}} \frac{1}{\sqrt{t-t^{\prime}}} d t^{\prime} .
$$

In this equation, $\mathrm{N}$ is the number of samples taken in the measurement, up to time $\mathrm{t}$. The smaller integrals are chosen such that, from $t_{i}$ to $t_{i+1}$, the temperature at the wall is known at the bounds of the integral from the measurement. The gradient of this temperature with respect to time can therefore be estimated for the period within the integral, by linear approximation. This can be solved exact for any measurement time, by evaluating all the separate integrals, and carrying out the summation

$$
q(t)=-\frac{k}{\sqrt{\pi \alpha}} \sum_{i=1}^{N-1} \int_{t_{i}}^{t_{i}+1} \frac{T_{i+1}-T_{i}}{\Delta t} \frac{1}{\sqrt{t-t^{\prime}}} d t^{\prime} .
$$

By evaluating equation 3-17 for all measurement times, the heat flux, as a function of time, is calculated for the whole measurement.

There is however one problem when applying this analysis method to the eroding thermocouple. This problem is that the required thermal conductivity and thermal diffusivity at the location of the junction are not known. This is most clearly seen when looking back at Figure 3.18. The location of the thermocouple junction can be at the middle of the mica layer, at the edge, or more likely: there can be multiple junctions, some on the mica and some at the edge of the mica. So the question is, what to use as thermal conductivity and thermal diffusivity in equation $3-17$ ?

The solution used by Xiaowei Wang [53], who used the eroding thermocouple for engine measurements was to calibrate the sensor using a laser excitation pulse. The problem with this method is that the absorption coefficient of the sensor is not known. Wang assumed this to be 1 , which is questionable. To avoid this assumption and the uncertainties it entails, an alternative calibration method was sought. 
The alternative calibration method uses the fact that the thermal conductivity and thermal diffusivity at the location of the junction appear as a constant in front of the integral in equation 3-17. The alternative calibration method consists of the following steps:

1. Preparing the eroding thermocouple junctions by grinding the junction in one specific direction. This ensures that all junctions are on one side of the middle mica layer, and not spread out as they would be when random grinding would be applied.

2. Measuring the surface temperature using the single shot reactor and sampling at a high sample rate (generally $200 \mathrm{kHz}$ ). This gives data with a sufficient temporal resolution that the discretization of the integral, as is done to arrive at equation 3-16, can be applied.

3. Filtering the surface temperature data, using a fifth order Butterworth filter. This gets rid of the bulk of the measurement noise.

4. Applying equation 3-17 with an arbitrary value for the thermal conductivity and thermal diffusivity of the material (for example, the thermal conductivity and thermal diffusivity equal to that of mica). This gives the shape of the heat flux in time, but not the actual absolute value.

5. Applying the found heat flux shape to a numerical grid, which simulates the eroding thermocouple. By comparing the found temperature profile on various points in the grid to the measured temperature profile, a scaling factor can be found, that scales the shape of the heat flux in time to the actual value of the heat flux in time.

6. Finally, as an extra check, the found heat flux can be applied to the grid again, to check whether the simulated temperature is the same as the measured temperature. 


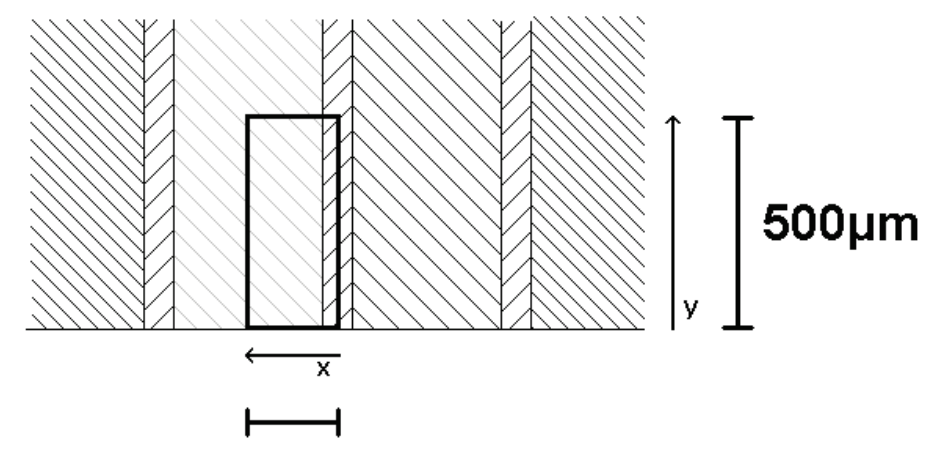

\section{$15 \mu \mathrm{m}$}

Figure 3.22, The calculation domain used for the numerical simulation

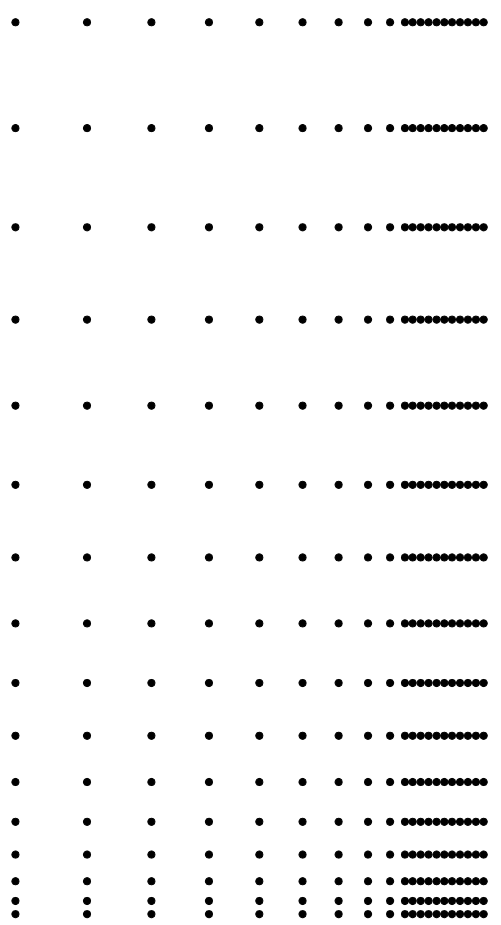

Figure 3.23, The calculation grid used for the numerical simulation

The numerical grid that is used in step 5 simulates part of the eroding thermocouple, making optimal use of symmetry. This is schematically shown in Figure 3.22. In Figure 3.23 the numerical grid is schematically shown. As can be seen, the grid is non uniform in both directions. In the y-direction 16 unevenly distributed nodes are used, the spacing between 
the node becoming larger further away from the edge. In both the mica part of the domain, as well as the metal part of the domain, 10 nodes are used. In the mica part they are evenly distributed, in the metal part spacing becomes larger further away from the mica.

On the grid the 2-D instationary diffusion equation is solved:

$$
\frac{\partial T}{\partial t}=-\alpha\left(\frac{\partial^{2} T}{\partial x^{2}}+\frac{\partial^{2} T}{\partial y^{2}}\right) .
$$

This is done by discretising the diffusion equation both in time and space. For the time discretization a simple Euler forward discretization is used, for discretization in space a central difference

$$
\begin{aligned}
\frac{T_{i+1}^{j, k}-T_{i}^{j, k}}{\Delta t}=-\alpha & \left(\frac{T_{i}^{j-1, k}-2 T_{i}^{j, k}+T_{i}^{j+1, k}}{\Delta x^{2}}\right. \\
& \left.+\frac{T_{i}^{j, k-1}-2 T_{i}^{j, k}+T_{i}^{j, k+1}}{\Delta y^{2}}\right) .
\end{aligned}
$$

The subscript $i$ indicates the discrete time step and superscripts $j$ and $k$ indicate the node number in $x$ and $y$ direction respectively. On the top and sides of the grid a symmetry boundary condition is used (so the gradient is kept equal to zero). This can be justified, for the sides, on the basis of symmetry and for the top based on the penetration consideration (heat from the hot gas does not penetrate to the end of the grid during the time scale of one cycle).

The grid can now be used in two ways: using the properties of chromel or using the properties of constantan. The properties of these two materials are taken from [56]. The properties of mica were supplied by the manufacturer of the eroding thermocouple. All properties as used in the numerical grid are summarized in Table 3.10.

\begin{tabular}{cccc}
\hline \hline Parameter & Mica & Chromel & Constantan \\
\hline Thermal conductivity, $\mathrm{k}\left[\mathrm{W} \cdot \mathrm{m}^{-1} \cdot \mathrm{K}^{-1}\right]$ & 0.67 & 17.3 & 22.7 \\
Density $\rho,\left[\mathrm{kg} \cdot \mathrm{m}^{-3}\right]$ & $2.9 \cdot 10^{3}$ & $8.7 \cdot 10^{3}$ & $8.9 \cdot 10^{3}$ \\
Specific heat, $\mathrm{C}_{\mathrm{p}}\left[\mathrm{J} \cdot \mathrm{kg}^{-1} \cdot \mathrm{K}^{-1}\right]$ & $0.39 \cdot 10^{3}$ & $0.43 \cdot 10^{3}$ & $0.40 \cdot 10^{3}$ \\
\hline \hline
\end{tabular}

Table 3.10, Properties of materials as used in the numerical simulation

Since the eroding thermocouple was ground in one specific direction, either using chromel properties, or using constantan properties will yield the correct solution for the heat flux. By doing two identical measurements, with the eroding thermocouple ground in different directions, it can be determined which of the grind directions will put the 
junctions on which material sides (which material was on which side was not indicated by the manufacturer).

\section{Example measurement analysis}

In Figure 3.24 the raw data of an experiment where the pressure in the launch chamber was initially 40 bar is shown. It can be seen that there is quite a significant difference in attained temperature, depending on the grind direction.

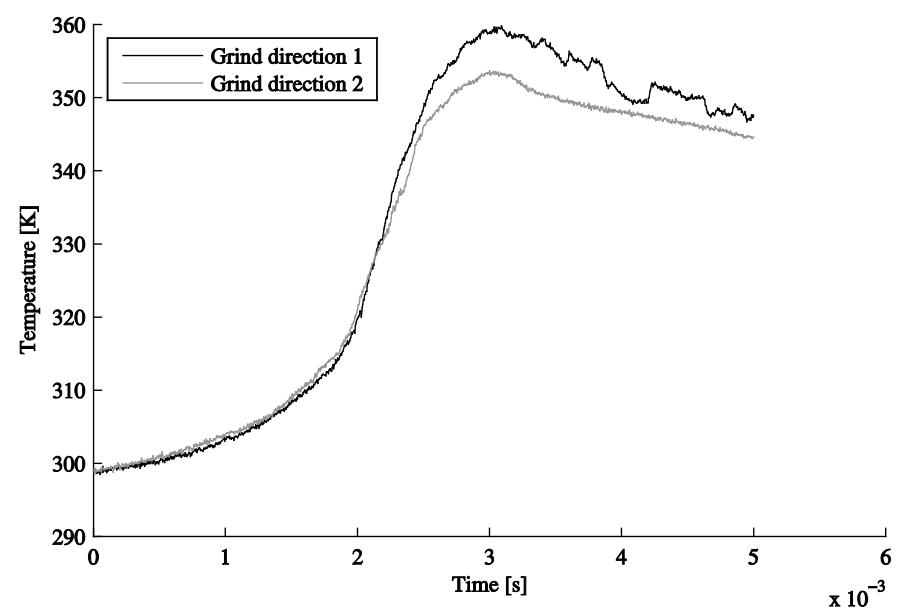

Figure 3.24, Example of the raw data obtained, initial pressure in launch chamber 40 bar

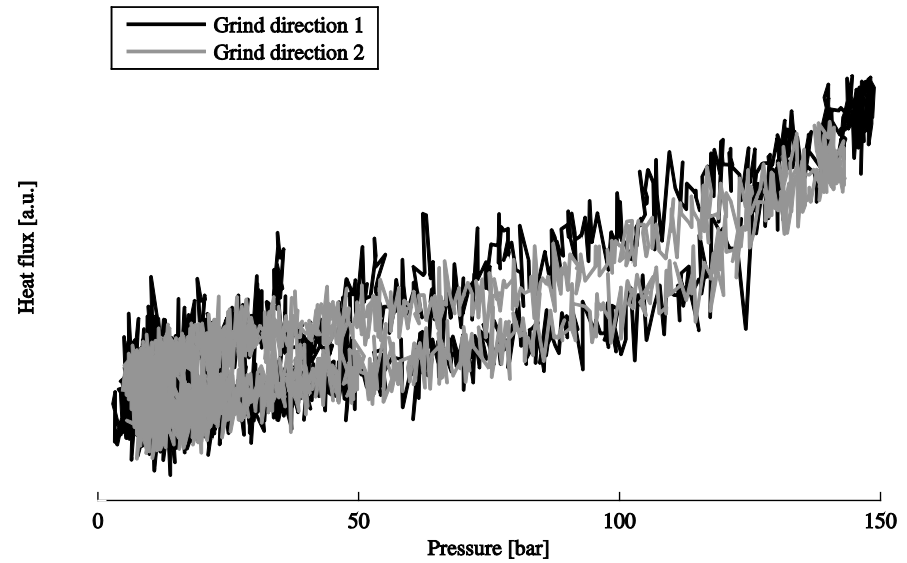

Figure 3.25, Heat flux obtained with unfiltered data from Figure 3.24 and arbitrary values for thermal conductivity and thermal diffusivity 
In Figure 3.25 the result obtained when step 4 is applied to the raw, unfiltered data shown in Figure 3.24. In this figure, there are no units on the $y$-axis, since arbitrary values were used for the thermal conductivity and thermal diffusivity of the material.

While the raw data presented in Figure 3.24 seemed quite free of measurement noise, it can be seen that the result obtained using this data unfiltered contains a great deal of measurement noise. In fact, the amount of noise is so high that the difference between the heat fluxes calculated for the different grind directions is not even possible. In Figure 3.26 the result is shown for the same experiments, this time using data that has been filtered using a fifth order Butterworth filter, using a cut off frequency of 0.05 times the sample frequency.

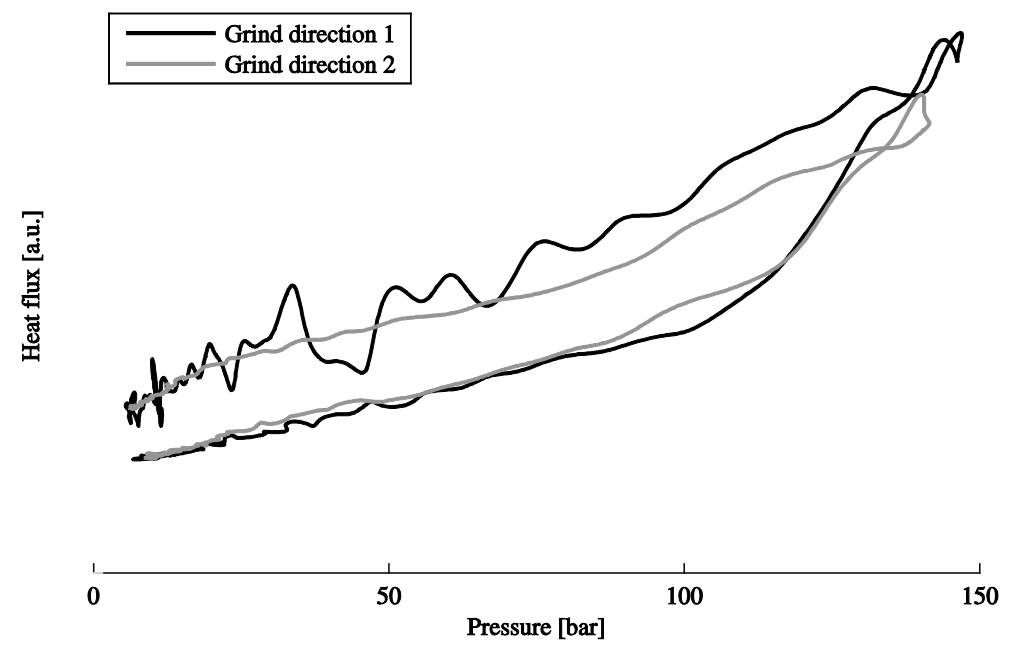

Figure 3.26, Heat flux obtained with filtered data from Figure 3.24 and arbitrary values for thermal conductivity and thermal diffusivity

In the results obtained with filtered data, the difference between the different grind directions is more evident. For the two calculated heat flux curves to overlap more accurately one would expect the grind direction 1 to be corrected by a lower correction factor than grind direction 2 .

In accordance with the steps, the found heat flux, with arbitrary units, should be applied to the numerical grid with either constantan or chromel properties. In Figure 3.27 the temperature distribution of the numerical grid containing chromel is shown, at the moment of highest temperature, when the heat flux calculated using the properties of 
mica as the arbitrary values required is used. In this figure the effect that the different properties of the materials have on the temperature distribution is visible. The temperature, mainly at the surface, of the mica is higher than the temperature of the chromel.

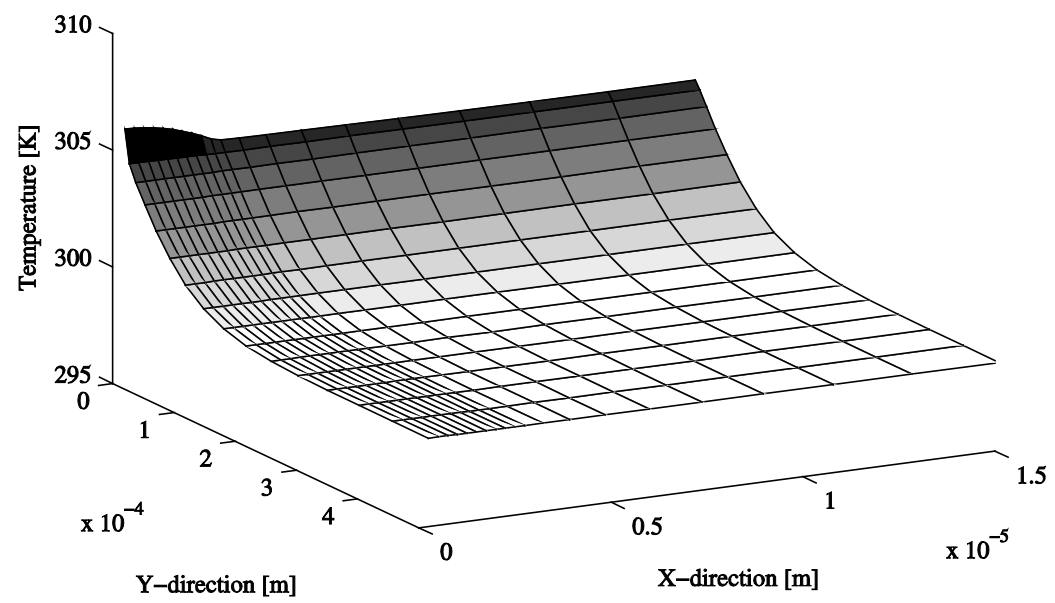

Figure 3.27, Temperature distribution in the numerical grid containing chromel, at the moment of highest temperature

Once the numerical simulation has been made, the simulated surface temperature can be compared to the measured surface temperature as shown in Figure 3.28. In this figure the simulated temperature in the middle of the mica, at the interface between chromel and mica and the average temperature of the simulated surface is shown. There is a significant difference between the measured and simulated surface temperature, when Figure 3.24 is compared to Figure 3.28. This difference is caused by the under prediction of the heat flux, since the properties mica were used for the calculation.

While the simulated temperature change is close to a factor ten smaller than the measured temperature change, the shape of the simulated temperature is a close duplicate of the (filtered) measured temperature, as was expected.

Now that the simulated temperature of the interface between the metal and the mica is known, it can be used to calculate the correction factor that needs to be applied to the calculated heat flux. This correction factor, when it is applied to the used material properties, can be seen as the effective material properties of the junction (the properties will logically be between the properties of the mica and the metal). 


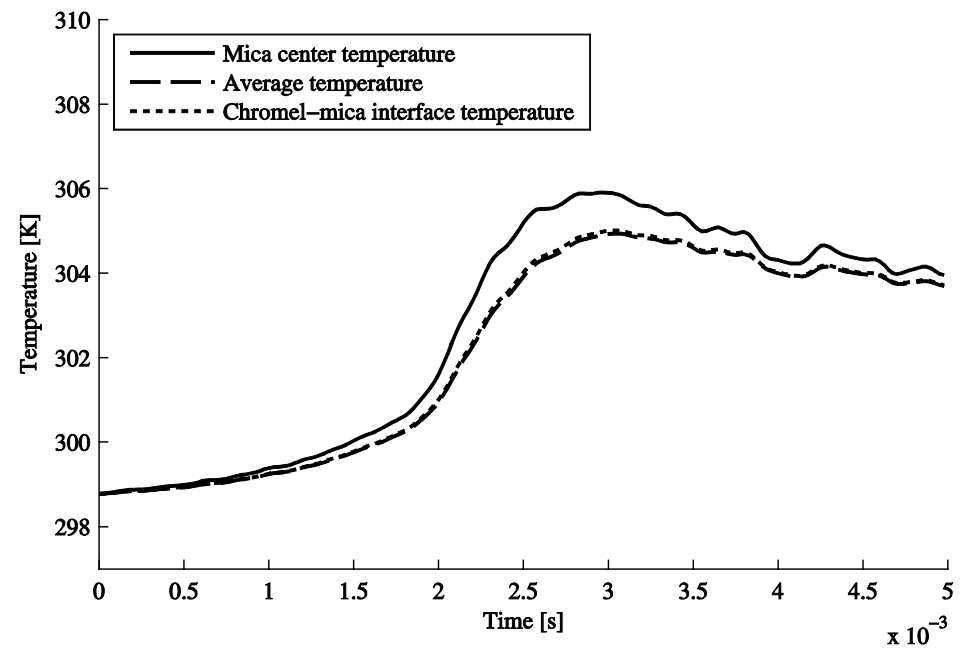

Figure 3.28 , Simulated surface temperatures compared to measured temperatures

In Figure 3.29 the correction factor, obtained from the three different simulated temperature profiles in Figure 3.28 are shown. The found correction factors using the average temperature and chromel-mica interface temperature are both relatively constant, which supports the expectation that the junction formed on the chromel-mica interface.

As a final check, the calculated heat flux (after application of the correction factor found) can be applied to the numerical grid again, to see whether the surface temperature of the interface between the chromel and mica now matches the measurement. This is shown in Figure 3.30. To obtain this figure, a correction factor of 9.68 is used: the average value of the calculated correction factor in Figure 3.29 over the last $90 \%$ of the calculated time (the first $10 \%$ is not used, because at that time the temperature change is negligible and the ratio of measured and simulated temperature change is greatly affected by small inaccuracies). 


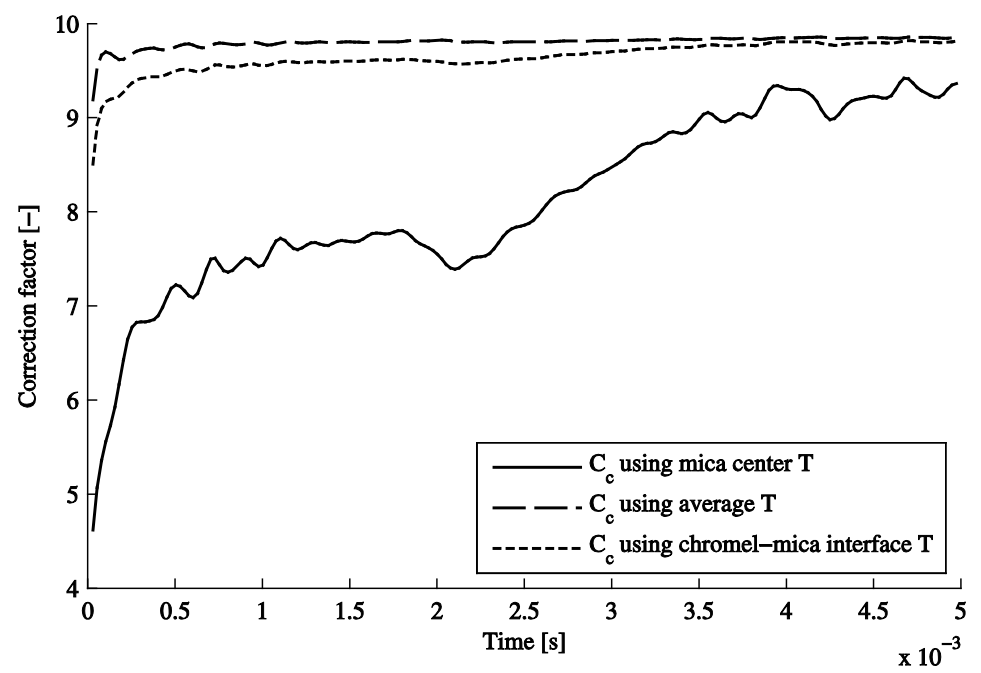

Figure 3.29, Correction factor based on different simulated temperatures

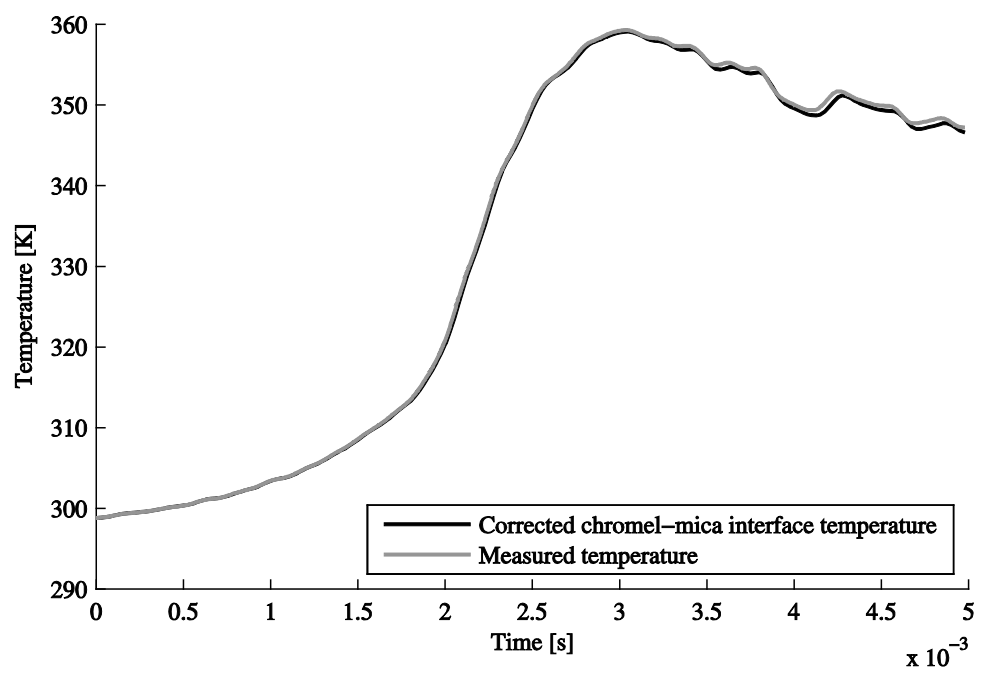

Figure 3.30, Chromel-mica interface surface temperature, calculated using the corrected heat flux, compared to the measured surface temperature

Now that a correction factor has been found, that correctly determines the effective thermal properties of the sensor; the actual instantaneous heat flux can be calculated. The only thing that remains is determining which grind direction puts the junctions on which material. As shown in the previous text and four figures, performing the analysis on a chromel grid gives a correction factor of 9.68 . This value is nearly the same for all 
experiments. When the same analysis is performed using a numerical grid containing constantan, a correction factor value of 10.80 is found. When looking at Figure 3.26, the logical conclusion is that grind direction 1 puts the junctions on the chromel side and grind direction 2 puts the junctions on the constantan side of the sensor.

\subsubsection{Velocity sensor data analysis method}

The signal that comes from the velocity sensor is a nearly continuous voltage signal, between 1 and 6 volts, which is a measure for the amount of light that is reflected by the piston surface. The light source that is used to measure the reflectivity of the surface has a frequency of $30 \mathrm{kHz}$, in order that the sensor can distinguish between the light that is reflected and stray light from other sources.

An example of the raw data that is obtained during the upward stroke of a piston is shown in Figure 3.31. In this figure $t=0$ is the moment of highest pressure.

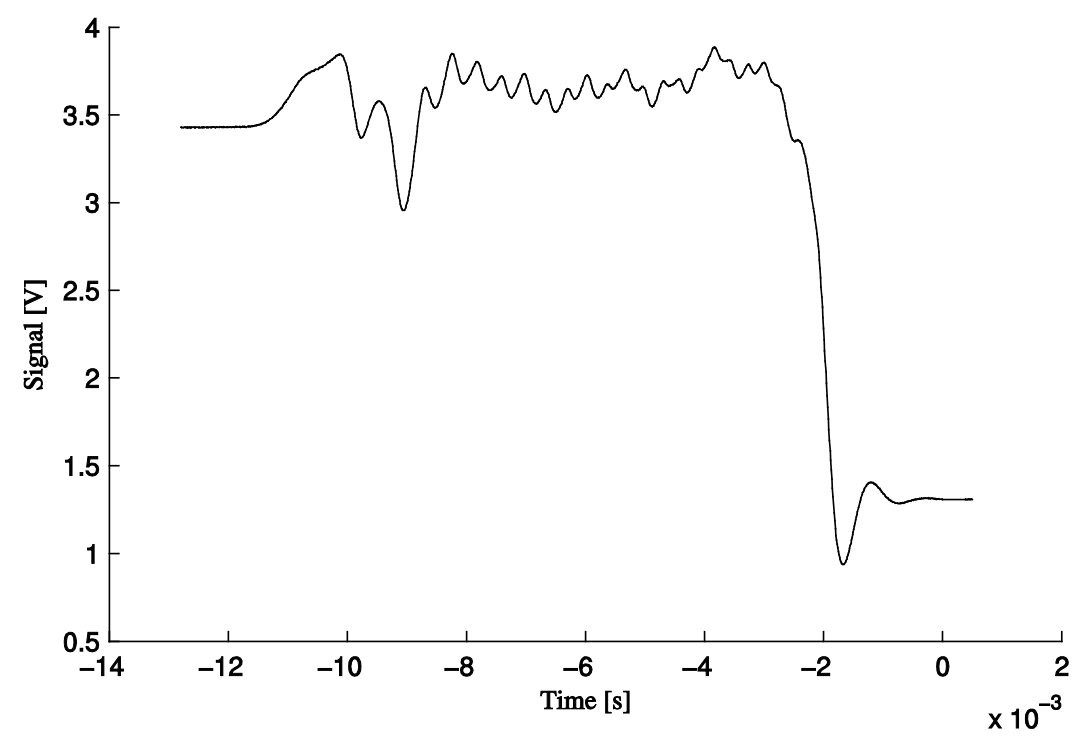

Figure 3.31, Example velocity sensor measurement signal

Figure 3.31 makes the main problem that the velocity sensor has clear. The frequency of the modulated light that is emitted by the sensor is $30 \mathrm{kHz}$, which means there is $33 \mu \mathrm{s}$ between different measurement points. However, if the piston travels at a velocity of $25 \mathrm{~m} / \mathrm{s}$ (which is typical for the reactor used), the time it takes for a groove to pass (the width of a groove being $0.3 \mathrm{~mm}$ ) is $12 \mu \mathrm{s}$. This means that the passing of grooves does not register as distinct events on the measurement signal. In order to improve the clarity 
of the signal, the signal is filtered and differentiated. In Figure 3.32 the filtered derivative of the raw data is shown. In this figure the local maxima and minima are indicated by dots. Each set of one maximum and one minimum show the passing of one groove. So, there are 44 maxima and minima for the 22 grooves in the piston.

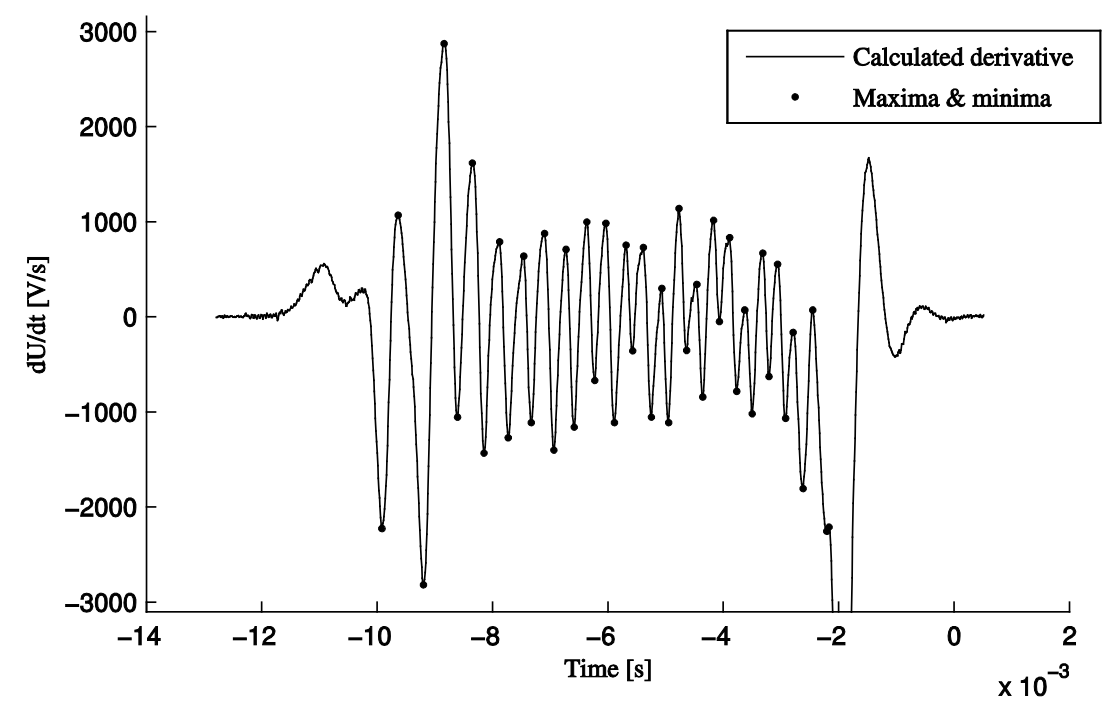

Figure 3.32, Derivative of the measurement signal, maxima and minima indicated

Taking a distance of $2.5 \mathrm{~mm}$ between the position of individual maxima and minima, the position of the piston can be plotted as a function of time. While this is in principle good enough to find the velocity of the piston as a function of time, a fit is used on the position data to derive the velocity. This is done because, as is shown in Figure 3.34, the directly calculated velocity from the position data contains too much noise. In Figure 3.33 the third order polynomial fit is shown, in Figure 3.34 the derivative with respect to time of this fit is shown. 


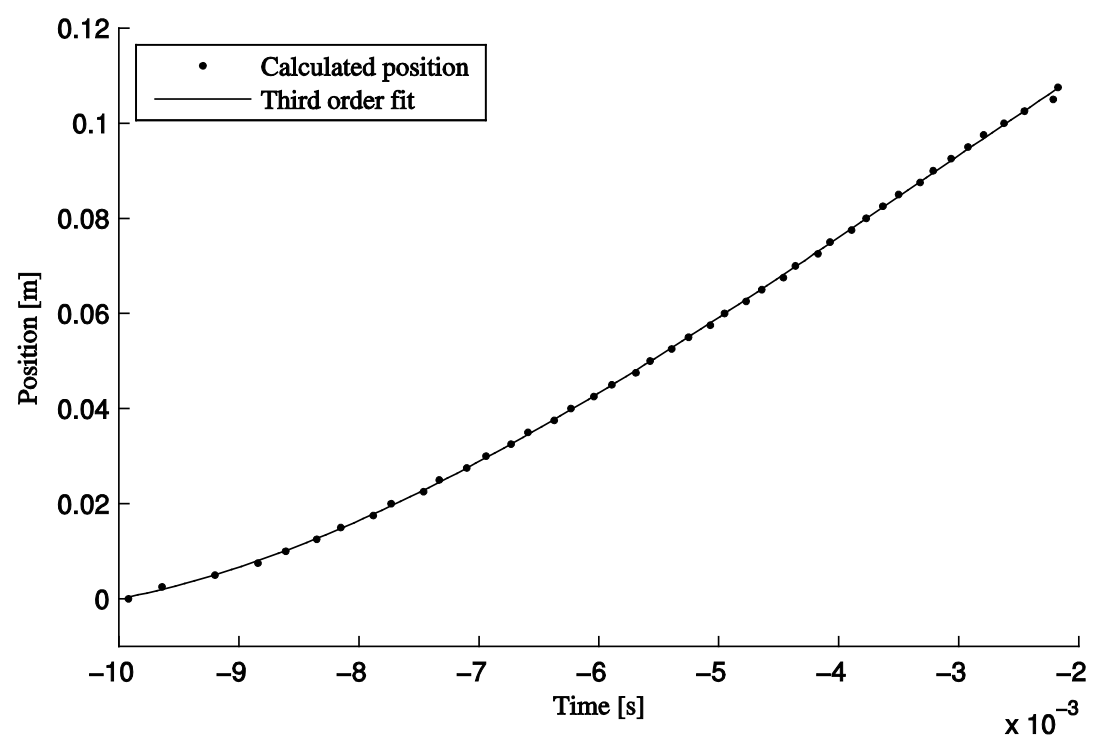

Figure 3.33, Calculated piston position data with third order fit

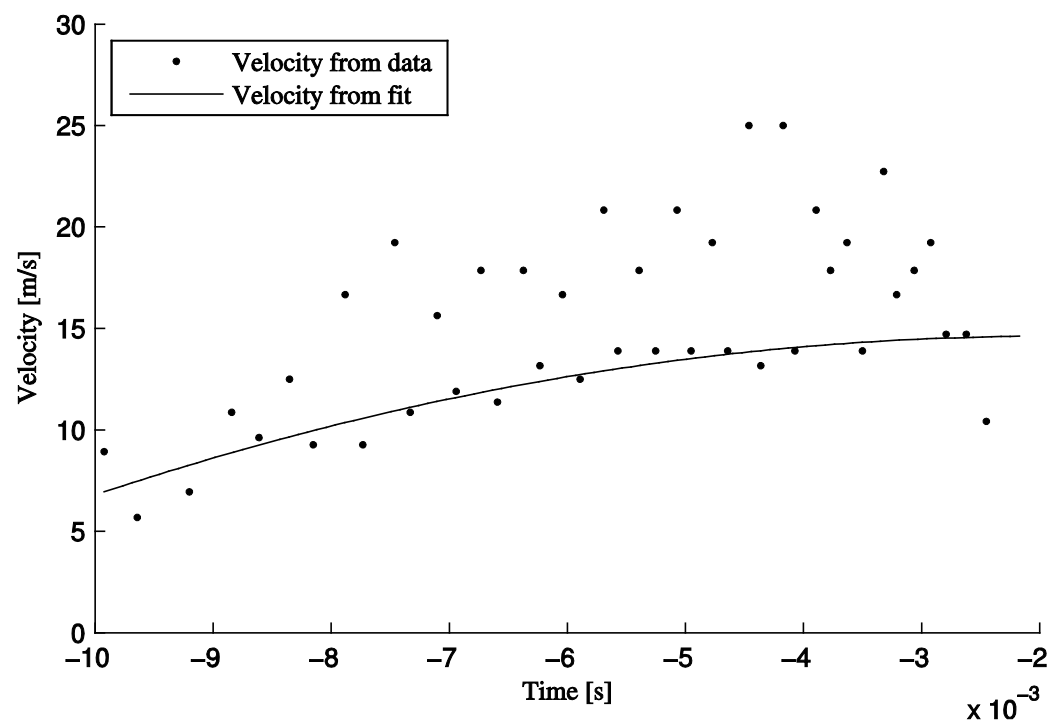

Figure 3.34, Calculated velocity directly from data as well as from fit

In order to make the velocity data of the piston more consistent and reliable, for the purpose of calculating the maximum energy of the piston, a linear fit is made on the last nine measurement points of piston position. From this linear fit a constant velocity is calculated. This can be seen as the average velocity the piston has during the last part of 
its stroke and is found to be more reliable than using the maximum velocity derived from the third order fit, since it is less sensitive to noise in the measurement data.

\subsubsection{Chemistry experiments experimental method and data analysis}

When the single shot setup is used for experiments with chemical reactions, the following experimental procedure is used.

Initially the piston is in the bottom of the reactor. Reactants are then supplied to the reaction chamber through one of the capillaries present in the top cover. Before the experiment a sample of the reactants in the reactor is taken. After this the compressionexpansion cycle is initiated by injection of compressed air at a predefined pressure through the launch mechanism. When piston motion has ceased, the products are extracted from the reactor. This is done by injecting air through the opening in the lower cover. This pushes the piston up and the products out of the reactor through the capillary tubes in the top cover. Both reactants and products are stored in gas sample bags that are leak tight against all reactants and products (including hydrogen) and analyzed using a gas chromatograph immediately after the experiments.

\section{Gas chromatograph}

The Gas Chromatograph used to analyze the reactant and product gasses from the experiments is a Varian CP-4900 micro-GC equipped with an MS5 and a PPQ column. The settings of the GC used to analyze the results are shown in Table 3.11.

\begin{tabular}{lcc}
\hline \hline Variable & MS5 & $\mathrm{PPQ}$ \\
\hline Carrier gas & Argon & Helium \\
Column temperature & $70{ }^{\circ} \mathrm{C}$ & $50{ }^{\circ} \mathrm{C}$ \\
Injector temperature & $80{ }^{\circ} \mathrm{C}$ & $80{ }^{\circ} \mathrm{C}$ \\
Injection time & $40 \mathrm{~ms}$ & $40 \mathrm{~ms}$ \\
& & $110 \mathrm{kPa}$ with a linear \\
Column pressure & $150 \mathrm{kPa}$ & ramp after $60 \mathrm{~s}$ of \\
& & $50 \mathrm{kPa} / \mathrm{min}$ to 200 \\
Sample frequency & \multicolumn{2}{c}{$\mathrm{kPa}$} \\
Sampling time & & $100 \mathrm{~Hz}$ \\
Measuring time & & $180 \mathrm{~s}$ \\
\hline \hline
\end{tabular}

Table 3.11, GC settings

\section{Correction for molar increase}

As the partial oxidation takes place, an increase in the number of moles in the reactor occurs. This can be seen from the overall reaction: 


$$
a \mathrm{CH}_{4}+\frac{a}{2} \mathrm{O}_{2}+b \text { inert } \rightarrow 2 a \mathrm{H}_{2}+a \mathrm{CO}+b \text { inert } .
$$

From this equation, the molar increase can be calculated. Since the gas chromatograph only measures molar concentrations of species in the product mixture, it is important to know the molar increase in order to be able to calculate the yield of products. However, it is likely that the reaction does not reach complete conversion, in which case one might calculate the molar increase from the hydrogen concentration:

$$
\begin{aligned}
& a \mathrm{CH}_{4}+b \mathrm{O}_{2}+c \text { inert } \\
& \qquad x \mathrm{H}_{2}+\frac{x}{2} \mathrm{CO}+\left(a-\frac{x}{2}\right) C H_{4}+\left(b-\frac{x}{4}\right) O_{2} \\
& +c \text { inert. }
\end{aligned}
$$

In this case, the fraction of hydrogen in the product can be expressed as

$$
\left[H_{2}\right]=\frac{x}{\frac{3}{4} x+a+b+c} .
$$

This can be rewritten to find the value of $x$, as a function of the measured hydrogen concentration:

$$
x=\frac{4\left[H_{2}\right](a+b+c)}{4-3\left[H_{2}\right]} .
$$

This result can then be used to calculate by which factor the number of moles in the reactor has increased, expressed as a function of the measured hydrogen concentration:

$$
f=\frac{a+b+c+\frac{3}{4} x}{a+b+c}=\frac{1}{1-\frac{3}{4}\left[H_{2}\right]} .
$$

The most important assumption that is made is that the reaction as shown in equation 3-20 is the dominant reaction path, and that different side reactions and side product concentrations are so small that they do not influence the molar increase significantly. Whether this assumption holds will be determined based on the results of simulations in section 5.7.4 .

\section{Conversion calculations}

To determine the success of the PCR in the partial oxidation or steam reforming of methane to syngas, the conversion of methane, as well as the yield of hydrogen and carbon monoxide need to be calculated. The conversion of methane is calculated by 
comparing the concentration of methane in the reactants to the concentration of methane in the products, using the previously determined molar increase factor:

$$
\zeta_{\mathrm{CH}_{4}}=1-\frac{f\left[\mathrm{CH}_{4}\right]}{\left[\mathrm{CH}_{4}\right]_{0}} .
$$

Via this definition a conversion of 1 means all methane has reacted to products and a conversion of 0 means no methane has reacted. To calculate the yield of hydrogen and carbon monoxide, the amount of product formed is compared to the amount that could hypothetically be formed from the methane present:

$$
Y_{\mathrm{H}_{2}}=\frac{f\left[\mathrm{H}_{2}\right]}{2\left[\mathrm{CH}_{4}\right]_{0}}, \quad \quad Y_{\mathrm{CO}}=\frac{f[\mathrm{CO}]}{\left[\mathrm{CH}_{4}\right]_{0}},
$$

These definitions are used for both the partial oxidation experiments, as well as the steam reforming experiments. This means that the yield of hydrogen in the case of the steam reforming experiments can be larger than 1 , since hydrogen can also be formed from the water present in the reactants.

\section{Carbon and oxygen balance}

By examining the carbon and oxygen balance of the process, an estimation of the species that cannot be identified by the GC can be made. By closing the carbon balance the missing carbon can be calculated. It is assumed that all the missing carbon is in the form of soot. This is justified by the lack of found signals in the GC spectrum, indicating that no other hydrocarbons are present. By closing the oxygen balance the missing oxygen can be calculated and the same can be done for the hydrogen. When the assumption is made that all missing oxygen and hydrogen is lost in the form of water vapor (which the GC cannot be calibrated for) the amount of water that is found to have formed from the oxygen balance can be compared to the amount of water that is found to have formed from the hydrogen balance. If these two amounts are the same, the assumption that all hydrogen and oxygen that is not accounted for is lost in the form of water is justified. Closing the carbon, hydrogen and oxygen balance is done by solving:

$$
\begin{gathered}
{[\mathrm{C}(\mathrm{s})]=\left[\mathrm{CH}_{4}\right]_{0}-f \cdot \sum_{i=1}^{N} x_{i} \cdot\left[\mathrm{C}_{x} \mathrm{H}_{y} \mathrm{O}_{z}\right]_{i},} \\
{\left[\mathrm{H}_{2} \mathrm{O}\right]_{\text {by } \mathrm{H}-\text { balance }}=4\left[\mathrm{CH}_{4}\right]_{0}+2\left[\mathrm{H}_{2} \mathrm{O}\right]_{0}-f \cdot \sum_{i=1}^{N} y_{i} \cdot\left[\mathrm{C}_{x} \mathrm{H}_{y} \mathrm{O}_{z}\right]_{i^{\prime}}}
\end{gathered}
$$




$$
\left[\mathrm{H}_{2} \mathrm{O}\right]_{\text {by } \mathrm{O}-\text { balance }}=2\left[\mathrm{O}_{2}\right]_{0}+\left[\mathrm{H}_{2} \mathrm{O}\right]_{0}-f \cdot \sum_{i=1}^{N} z_{i} \cdot\left[\mathrm{C}_{x} \mathrm{H}_{y} \mathrm{O}_{z}\right]_{i}
$$

\section{Correction for enclosed air}

In connecting and disconnecting the sample bags used for the collection of products to the setup and the gas chromatograph, some air is enclosed in the bag valve. To correct for this, it is assumed that the amount of enclosed air is $3.7 \%$ of the total volume of the sample in the bag. This number is based on experiments with pure nitrogen, where an average oxygen concentration of $0.79 \%$ was measured. The correction of the oxygen concentration is done according to

$$
\left[\mathrm{O}_{2}\right]_{\text {corrected }}=\frac{\left[\mathrm{O}_{2}\right]-0.79 \%}{1-0.037}
$$

and the other species are corrected according to

$$
[X]_{\text {corrected }}=\frac{[X]}{1-0.037}
$$




\section{Heat transfer}

This chapter is concerned with some of the heat transfer as it occurs in the PCR. While there are many forms of energy losses from the PCR, one is discussed in particular in this chapter. This is the heat transfer, from the hot (reaction) chamber to the surrounding walls and piston. The heat transfer is investigated to considerable detail. The reason is that heat loss (or gain) to the walls, will also influence the chemistry.

The investigation of the heat transfer has been divided into two parts: averaged heat transfer, and instantaneous heat losses. The averaged heat transfer concerns the heat that is transferred to the walls during continuous, steady reciprocation of the piston. The instantaneous heat losses are the heat losses that occur in the single shot reactor during one reciprocation.

\subsection{Introduction}

The influence that heat transfer can have on the functioning of the PCR make that it is vital to acquire some knowledge in this area. Since the PCR is still in its infancy stage, robust measurement methods have to be developed that can be applied to the PCR and provide information on heat transfer. This makes the goals of the heat transfer experiments the following:

1. To develop an accurate and robust method for measuring the average transfer rate from the gas to the reactor walls and piston, for different operating conditions.

2. To test this method by measuring the averaged heat flux at various operating conditions and using this flux to calculate a heat transfer coefficient.

3. To find an empirical correlation between the heat transfer coefficient and the peak compression pressure in the reactor.

4. To develop an accurate and robust method for measuring the instantaneous heat flux from the PCR, within one cycle, for different operating conditions

5. To test this method by measuring the instantaneous heat flux at various operating conditions.

6. To arrive at an empirical relation between pressure and heat flux. 


\subsection{Heat transfer}

In order to get a feel for the expected results from the heat transfer experiments one can take a look at the theory and make a prediction of what the results will look like. This enables the evaluation of any results obtained in a later stage, and will show whether the results obtained agree with the theory.

\subsubsection{Heat transfer theory}

The driving force behind the heat flux from the reacting gasses to the reactor body (and vice versa) is the temperature difference between the two. The value of the heat flux is determined by the gradient of the temperature at the interface between the gas and the reactor walls:

$$
q(t)=-\left.k_{w} \frac{\partial T_{w}}{\partial x}\right|_{x=x_{w 0}}=-\left.k_{g} \frac{\partial T_{g}}{\partial x}\right|_{x=x_{w 0}} .
$$

In this equation the notation $x_{w 0}$ indicates that the gradient is taken at the edge of the wall ("wall" being used here to indicate either the reactor body or the piston). To calculate the heat flux, the gradient can be taken either in the wall, using the thermal conductivity constant of the wall, or taken in the gas, using the thermal conductivity constant in the gas, since energy conservation dictates the two energy fluxes are the same.

So, the heat flux depends on the size of the temperature gradient at the interface and the thermal conductivity. There are several important factors that determine the size of the gradient at the interface. These factors can most easily be identified at the hand of Figure 4.1. In this figure, the temperature distribution is shown along a line perpendicular to the interface. This temperature distribution in the gas is divided into two parts: an area where the influence of the wall is not felt and an area where the presence of the wall influences the temperature. Similarly, the temperature in the wall is divided into a part where the influence of the gas is felt, and an area where the influence of the gas is not felt. 


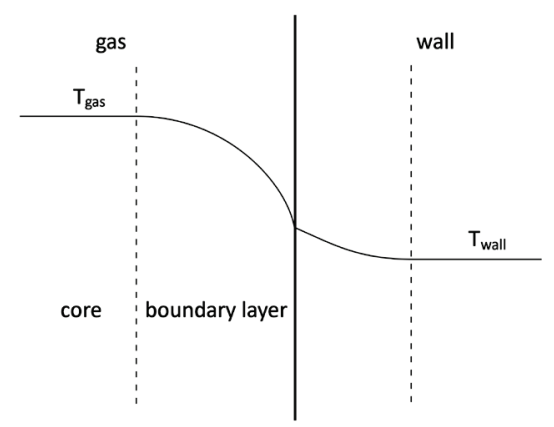

Figure 4.1, Schematic temperature distribution

Figure 4.1 illustrates the different factors that have an influence on the gradient of the temperature at the interface between the gas and the wall. These are most importantly, the temperature difference between the core of the gas and the bulk of the wall: a higher temperature difference will logically also induce a higher gradient at the wall, and the thickness of the boundary layer: a thinner boundary layer will also give a higher gradient at the wall. These are in turn influenced by various factors. The most important of these factors are:

- Initial gas temperature

- Compression ratio

- Compression rate

- Presence of initial gas motion

\section{Initial gas temperature}

As follows from the analysis on compressive heating made in section 2.3.2, a higher initial gas temperature will, at the same compression ratio, result in a higher compression temperature. So, if the walls of the reactor are not preheated to the same higher initial gas temperature, significantly more heat will be lost. If however, the walls of the reactor are also preheated, to the same temperature as the gas, this effect is somewhat diminished. This can be seen from the following analysis.

If a volume of argon, with initial temperature of $293 \mathrm{~K}$, is compressed by a factor of 3.17, the final adiabatic temperature of the argon exactly doubles, according to: 


$$
\left(\frac{V_{0}}{V(t)}\right)^{\gamma-1}=\frac{T(t)}{T_{0}} .
$$

So the temperature difference between the core gas and the bulk of the wall will be 293 $\mathrm{K}$. If both wall and gas are now preheated by $100 \mathrm{~K}$, to 393 , the final gas temperature will be, for the same compression ratio, twice $393 \mathrm{~K}$. So, the temperature difference between the core gas temperature and the wall bulk will be 393 . This means that preheating both the gas and the wall by an equal amount will still increase the temperature difference for equal compression.

\section{Compression ratio}

The effect of compression ratio on the temperature difference is threefold. Firstly, looking again at equation 4-2, it can be seen that, for argon, an increase of the compression ratio from 3.17 to 6.24 , will cause the temperature increase to go from doubling for the 3.17 compression ration case, to tripling at the 6.24 compression ratio case.

A second effect that the compression ratio has on the heat transfer rate is that, if a gas is compressed, the boundary layer is also compressed. This means that the distance between the core gas and the wall is smaller, thus increasing the gradient of the temperature at the interface.

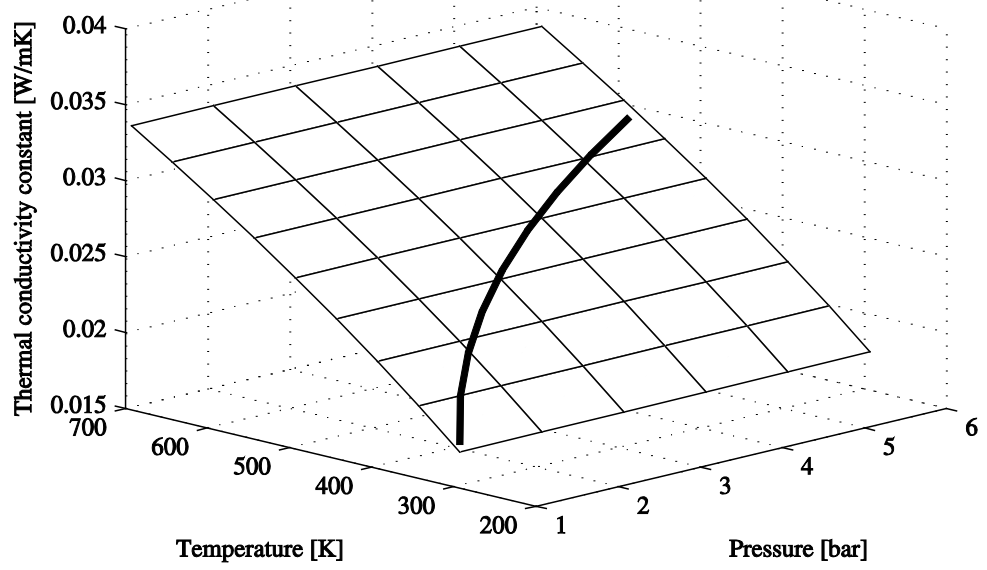

Figure 4.2, Thermal conductivity as a function of temperature and pressure

The third effect is the effect that compression has on the thermal conductivity of the gas. As the gas is compressed, its thermal conductivity increases. While good semi empirical 
relations are available for mono- and poly-atomic gasses at low pressures (such as the Chapman-Enskog theory [57] and Euken formula [58]), at high pressures one has to rely on (extrapolated) experimental data or empirical relations based on experimental data, such as the NIST database [59]. In Figure 4.2, data taken from NIST is plotted for argon, as a function of temperature and pressure. Superimposed on this data is a simple compression-expansion curve, with a compression ratio of 3.17. Since the data from NIST does not go beyond $700 \mathrm{~K}$, data needed for higher compression ratios, will have to be taken from a different source, or extrapolated. Even though the NIST database may be limited, the clear trend can be seen: as the pressure and temperature of the argon is increased, the thermal conductivity also increases.

The total effect of the compression ratio on the heat transfer is the sum of the three different aspects. So the final effect is that a higher compression ratio increases the heat losses.

\section{Compression rate}

While the effects of initial gas temperature and compression ratio are clear and conclusive, the compression rate is more complicated. To get an idea of the effect of compression rate on the heat loss, research done on rapid compression machines can be used as a reference.

Several designs for the RCM have been made in the past by different researchers. In 1969 it was shown by Tabaczynski [60] in a simple setup that the motion of a piston along a wall scrapes off gas from the walls of the chamber. This creates a so-called torroidal roll-up vortex that increases in size during a compression stroke. A sketch of the roll-up vortex can be seen in Figure 4.3.

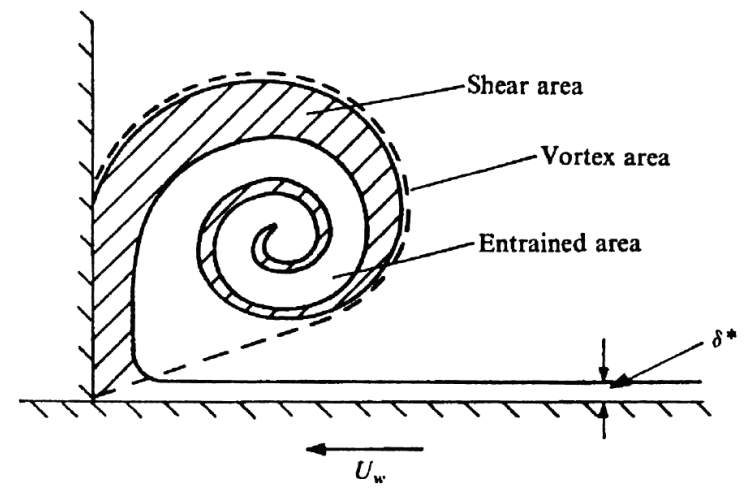

Figure 4.3, Schematic representation of the roll-up vortex [60] 
The RCM used by Griffiths et al. [61] compressed gas mixtures with volumetric compression ratio up to 15 . They determined the development of the temperature field inside the reactor chamber and observed that the roll-up vortex moves "cold" gas from the wall into the hot core. At the end of compression, this resulted in two regions. The first is a region with adiabatically heated gas, taking up to $70 \%$ of the volume (the 'adiabatic core'). This adiabatic core has uniform properties. The other part is a boundary layer region, in which the properties are not uniform and which is not heated adiabatically, similar to the situation depicted in Figure 4.3.
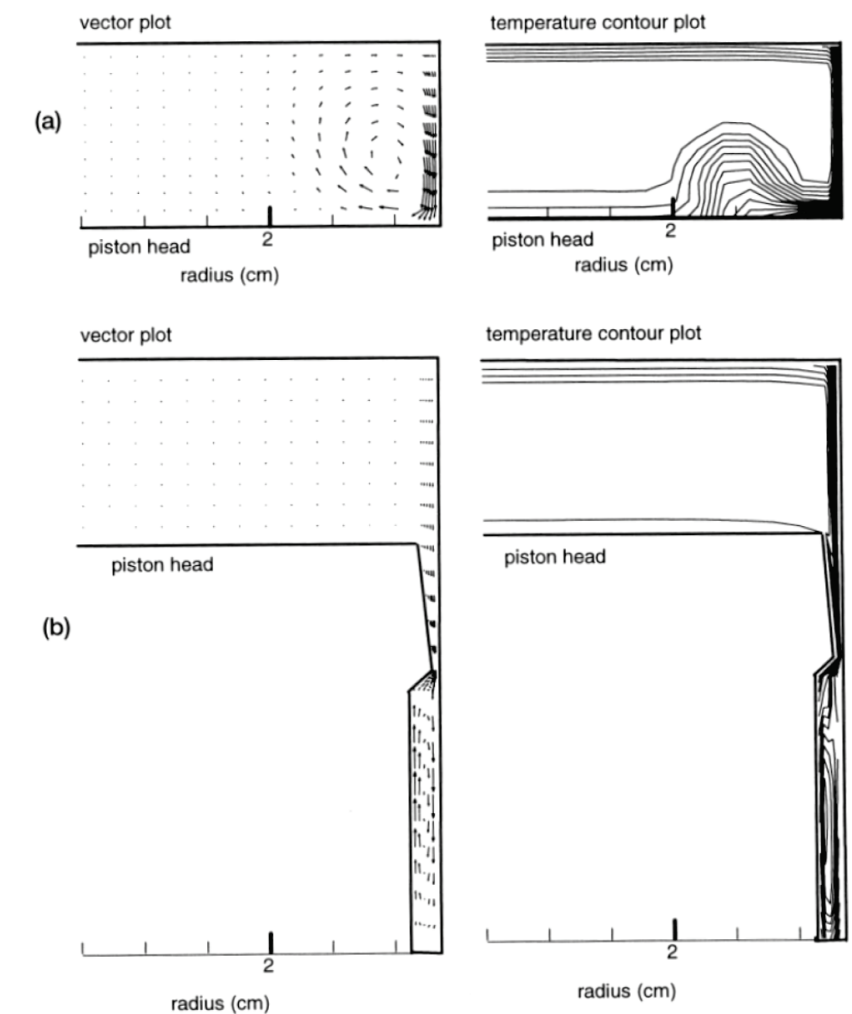

Figure 4.4, Numerical simulations of the flow- and temperature field in a rapid compression machine [62]

This division of the gas in two regions is even clearer in the work of Lee and Hochgreb [62]. They made numerical simulations of the roll up vortex during compression. An important conclusion that came from their work is that the roll up vortex increases heat transfer to the wall. This is most clearly visible in Figure 4.4, where an attempt is shown 
to reduce the effect of the roll up vortex by using a creviced piston. In the temperature plot it is visible that the size of the adiabatic core is much larger in the case where the roll up vortex is suppressed, indicating that less energy was lost to the walls.

Since an increase in compression rate is attained by increasing the piston speed, thus increasing the average velocity of the gas within the chamber, it is likely that a higher compression rate, will induce a higher heat transfer rate, by the increased gas motion. However, on the other hand, a cycle with a higher rate of compression will likely also have a higher rate of expansion, and thus a shorter total cycle time. And a shorter cycle time means that there is less time for which heat is lost. So, the total heat loss, the integral of the heat flux over time, may in fact be smaller, while the instantaneous heat flux is higher. Which of the two effects will be prevailing would have to be seen experimentally.

\section{Initial gas motion present}

The effect of the initial gas motion is quite similar to that of the induced gas velocity of the piston motion. Any initial gas motion in the chamber, caused by for example the high velocity injection of fuel and oxidizer, will to some extent help mix the cold boundary layer with the hot core. So it can be expected that, for example, a higher injection velocity of reactants will cause a higher heat transfer rate. Whether this effect is very large is however quite doubtful. Again, this would have to be ascertained by either experiments or detailed simulations.

\subsection{Continuous heat transfer}

Using the setup described in section 3.2 and the data analysis procedure described in 3.2.4 a series of measurements is done, to determine the average heat transfer rate in a continuously reciprocating PCR. In Figure 4.5 the curves that were constructed from a series of measurements with static supply line pressures ranging from 5 to 15 bar is shown. In the analysis a linear fit through the measurement points was used to obtain the average heat flux through the measurement domain and the error function fit was used to obtain the surface heat flux.

As mentioned in the description of the setup, experiments using higher pressures are only possible for short times, since the supply of high pressure air is limited. A second problem that manifested itself during the measurements is the temperature change that occurred in the reactor, caused both by the heating effect of the compression and expansion of the gas in the top of the reactor, as well as the cooling effect that the cold injected air had on the bottom of the reactor. This caused the reactor to stop running 
smoothly a short time into the experiment. The result is that relatively short heat flux curves for the measurements with a static driving pressure of 7.5 to 15 bar were obtained.

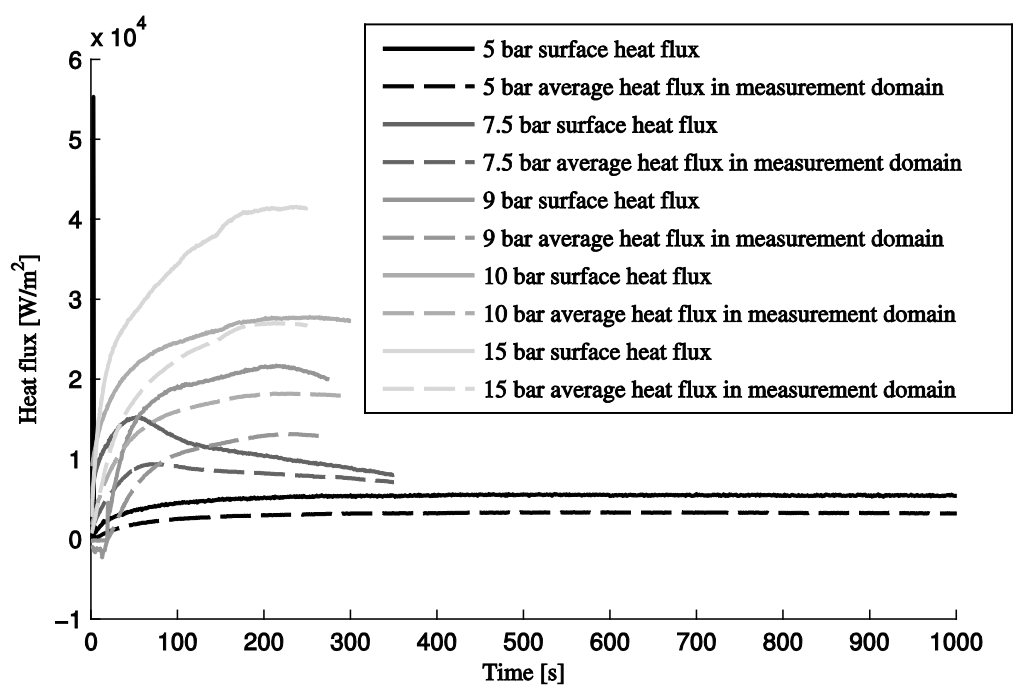

Figure 4.5, The determined average heat flux, at different static driving pressures as a function of time

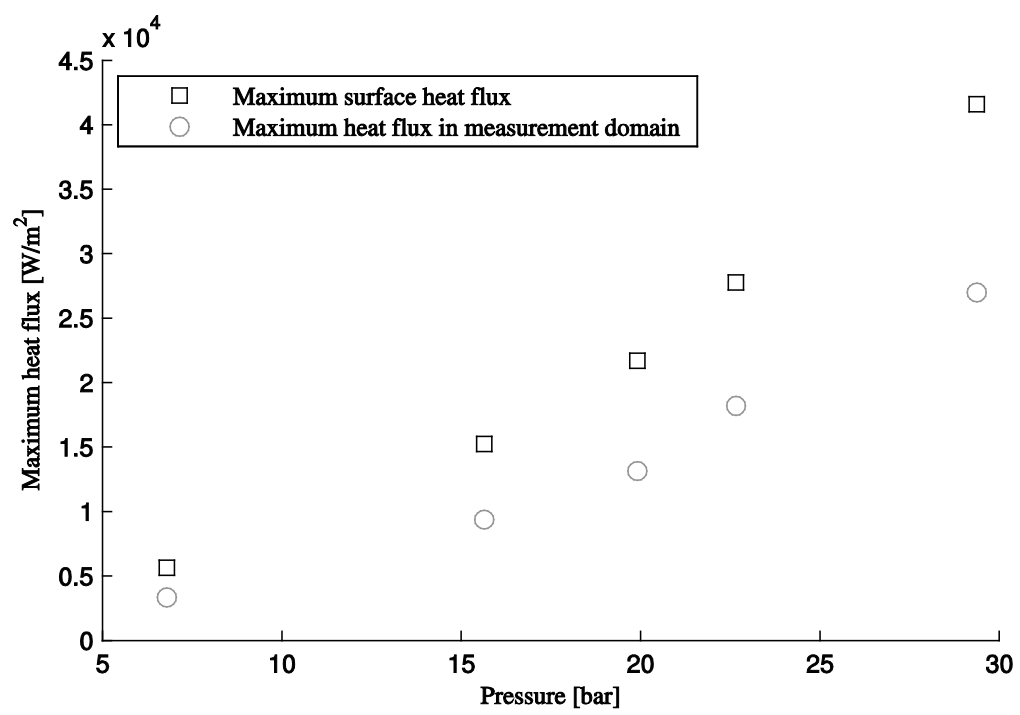

Figure 4.6, The maximum determined averaged heat flux, at different peak compression pressures 
While the experiment with a driving pressure of 5 bar does reach steady state, the other experiments in the series clearly do not. However, a maximum measured heat flux can be determined for all experiments. In Figure 4.6 the maximum determined heat flux for each of the experiments, plotted as a function of maximum compression pressure is shown.

The acquired data can be used to calculate a heat transfer coefficient as outlined in section 3.2.4. As mentioned in section 3.2.4 the used definition of the heat transfer coefficient is

$$
q(t)=h(t)\left(T_{a d-\max }-T_{w}(t)\right) .
$$

Doing this for the measurements, a heat transfer coefficient as a function of time, as shown in Figure 4.7, is found.

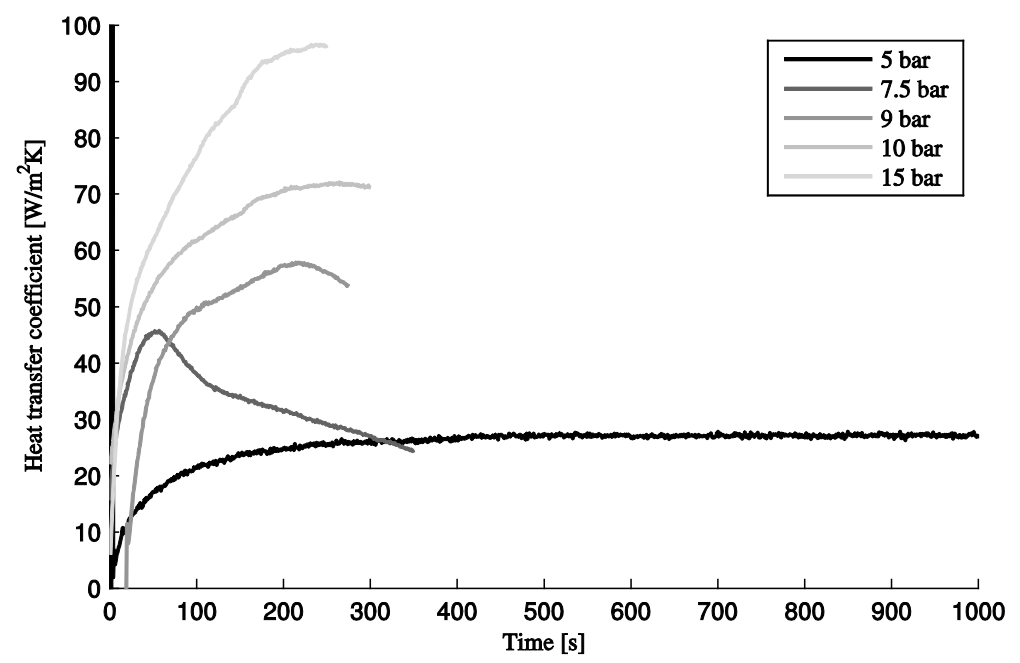

Figure 4.7, The determined heat transfer coefficient, for different static driving pressures as a function of time

In Figure 4.8 the maximum heat transfer coefficient for each of the experiments is plotted as a function of the peak compression pressure. From the data in this figure empirical relation can be derived, forming an upper bound of the average heat transfer coefficient as a function of compression pressure. The function

$$
h=a \cdot P^{b}+c,
$$


is used for fitting. This function is chosen purely for convenience reasons, it is not implied that the pressure is in fact the only determining factor that determines the heat transfer coefficient. In Figure 4.8 the maximum heat transfer coefficient is shown, together with the determined fit. This fit only applies to the reactor used. If a reactor with a different geometry, parts made from different materials, compressing a different gas or operating in a different compression regime, is used, the empirical relation does not apply. The found fitting constants are shown in Table 4.1.

\begin{tabular}{lccc}
\hline \hline Fit constant & $\mathrm{a}$ & $\mathrm{b}$ & $\mathrm{c}$ \\
Value & 0.18 & 1.78 & 21.9 \\
\hline \hline
\end{tabular}

Table 4.1, Determined fitting constants

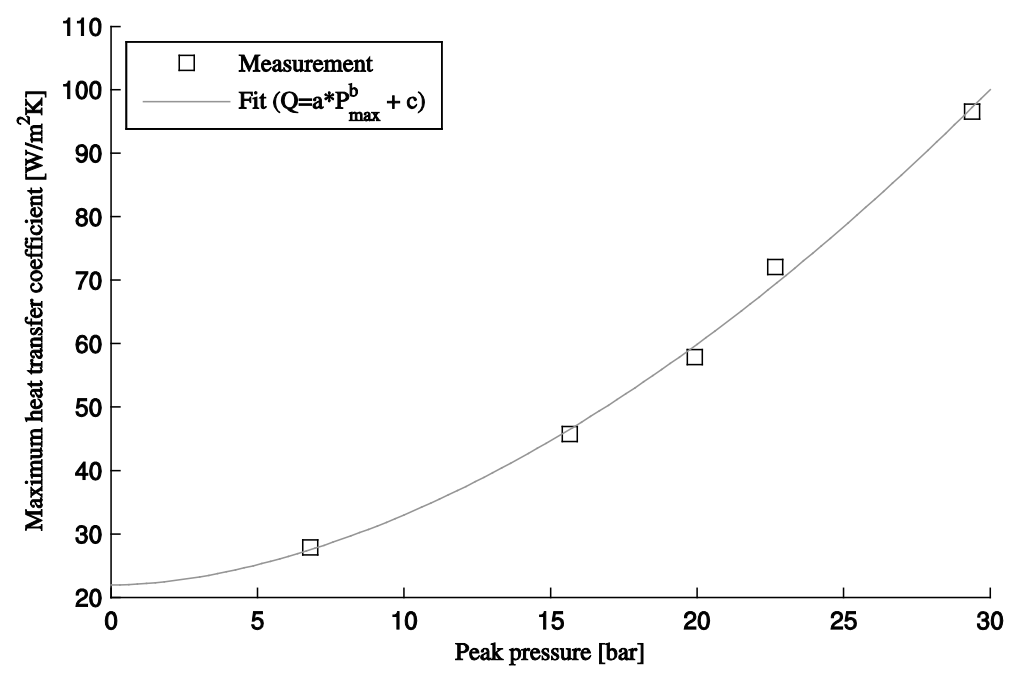

Figure 4.8, The maximum determined heat transfer coefficient, at different peak compression pressures, with the determined fit

The found heat transfer relation can be used to estimate the heat loss in this particular case, during a compression expansion cycle. This can be compared to the energy put in to compress the gas, to give an idea of the energy losses of this particular reactor, when it is operated as done in the experiment. For the 5.0 bar static driving pressure case a total heat loss of about $31 \mathrm{~W}$ is found (using $\mathrm{q}=5.6 \cdot 10^{3} \mathrm{~W} / \mathrm{m}^{2}$ and the heat transfer area $A=5.5 \cdot 10^{-3} \mathrm{~m}^{2}$, which includes the portion of the sides of the reactor that is exposed to the high temperatures). 
This means that at an operating frequency of $f=51 \mathrm{~Hz}, 0.56 \mathrm{~J}$ of energy is lost per cycle. This value can be compared to the work done on the gas to compress it:

$$
W=\frac{P_{0} V_{0}}{\gamma-1}\left[\left(\frac{V_{0}}{V}\right)^{\gamma-1}-1\right]
$$

where the different volumes are found using the ideal gas law. The work done is found to be $\mathrm{W}=48.4 \mathrm{~J}$, which means that, for this particular case, about $1.2 \%$ of the energy put in is transferred to the reactor cover, and is not used for the compression of the next batch of reactants. Of course, in reality, many factors influence the heat loss from the reactor, and in reality the heat lost may be much lower than the calculated value for this particular case.

\subsection{Instantaneous heat loss}

The single shot setup, as described in section 0 , is used for a series of instantaneous heat flux measurements. The measurements are performed at three different launch pressures. Each launch pressure was used six times, three times with the temperature sensor ground in the first direction and three times with the temperature sensor ground in the opposite direction. In Table 4.2 the settings of the launch system are shown, as well as the resulting maximum piston velocity (averaged over the six measurements).

\begin{tabular}{cccc}
\hline \hline $\begin{array}{c}\text { Launch pressure } \\
\text { (bar) }\end{array}$ & $\begin{array}{c}\text { Trigger pressure } \\
\text { (bar) }\end{array}$ & $\begin{array}{c}\text { Counter pressure } \\
\text { (bar) }\end{array}$ & $\begin{array}{c}\text { Averaged maximum piston } \\
\text { velocity }\left(\mathrm{m} \cdot \mathrm{s}^{-1} \text { ) }\right.\end{array}$ \\
\hline 40 & 40 & 10 & 17.5 \\
50 & 50 & 15 & 18.9 \\
60 & 60 & 20 & 20.1 \\
\hline \hline
\end{tabular}

Table 4.2, Settings launch system and resulting maximum piston velocity

For each of the in total eighteen measurements, the heat flux is calculated according to the data processing method outlined in 3.3.5. The corrected heat flux during the investigated reciprocation, at various pressures is shown in Figure 4.9 (of each of the launch pressures and grinding directions only one result is shown). 


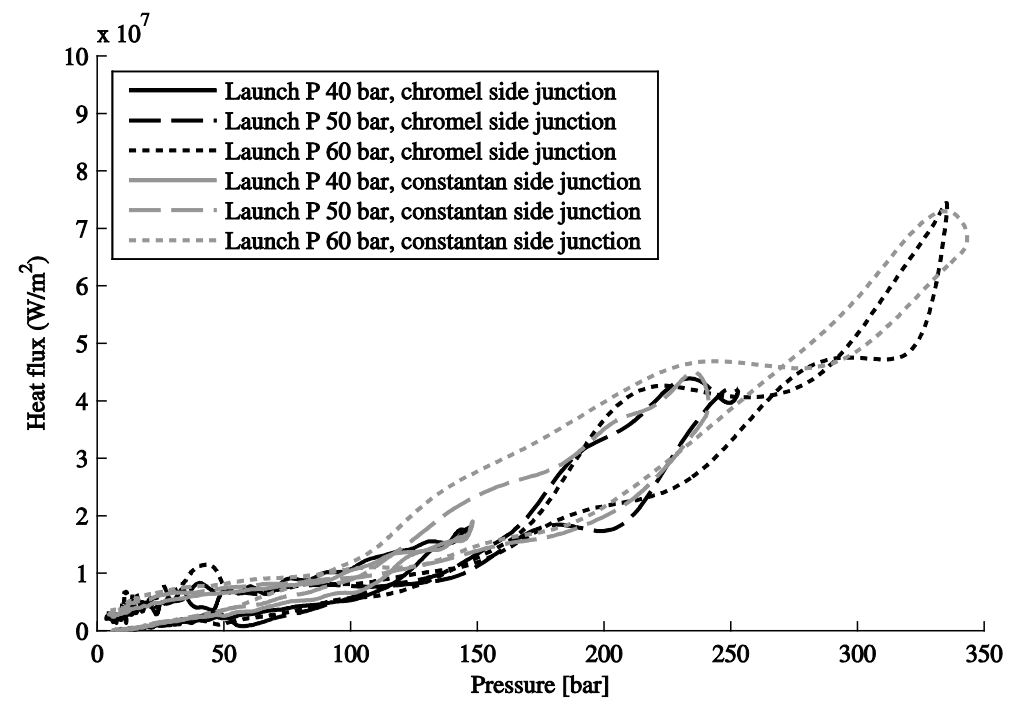

Figure 4.9, The determined instantaneous heat flux, at different launch pressures, with the junction on the two different sides

From these results an exponential relation between the heat flux and the pressure is visible, which is what is generally found in literature. It is visible that the found heat fluxes using the different grind directions now perfectly coincide, thanks to the correction procedure. This supports the use of the proposed procedure, as opposed to laser calibration procedures used by Wang et al [53].

\subsubsection{Modeling the observed results}

In order to make the results usable for future work, for example in modeling the single shot reactor, a good fit needs to be made through the data obtained. This is done by using the upstroke data of the six measurements shown in Figure 4.9. Since these measurements have a lot of data in the low pressure region and relatively little data in the high pressure region (caused by the fact that data acquisition is done at regular intervals), a new data set is created for each measurement. This set contains interpolated data points, equally spaced on the pressure axis. This ensures that there is no uneven bias on the low pressure part of the measurement in the fit. The function

$$
q=P^{a}+b \cdot P,
$$

is used for fitting. This function is chosen purely for convenience reasons, it is not implied that the pressure is in fact the only determining factor that determines the heat flux. One fit is made for each of the measurements and then the six found fitting constants 
are averaged to generate the two constants needed to make one unique fit. This final fit, including the data used for fitting is shown in Figure 4.10.

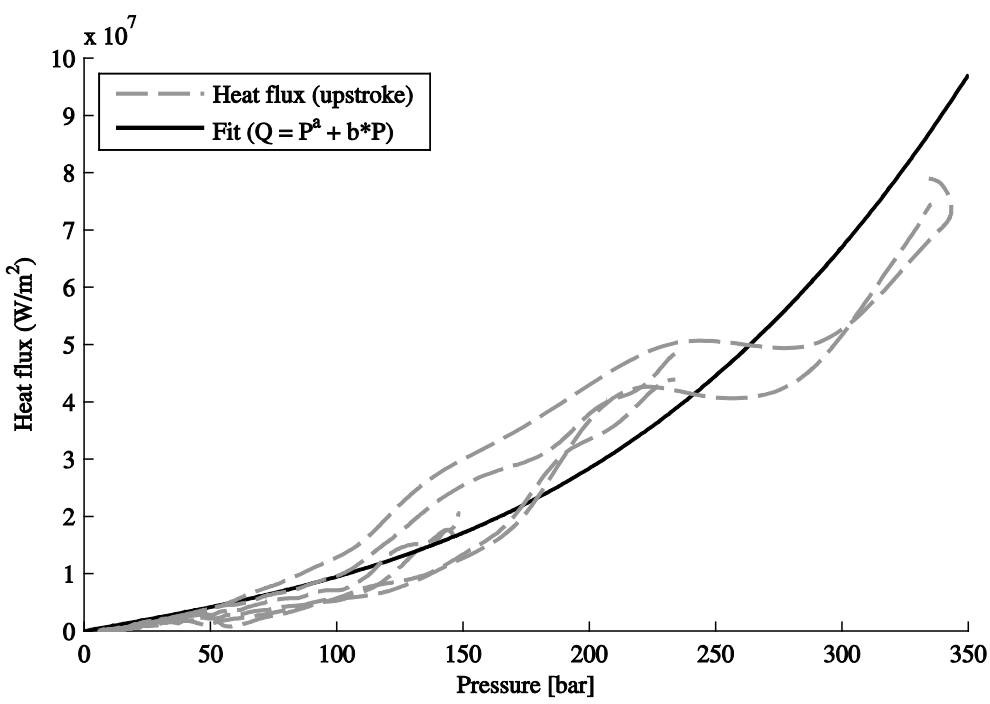

Figure 4.10, The determined instantaneous heat flux, during the upstroke, at different launch pressures, with the junction on the two different sides and the resulting fit.

The fit coefficients that were found, both for the six separate measurements, as well as their average, is shown in Table 4.3.

\begin{tabular}{ccc}
\hline \hline Measurement & $\mathrm{a}$ & $\mathrm{b}$ \\
\hline $\mathrm{P}_{1}=40$, Ch side junction & 3.30 & $0.24 \cdot 10^{5}$ \\
$\mathrm{P}_{1}=50$, Ch side junction & 3.22 & $0.30 \cdot 10^{5}$ \\
$\mathrm{P}_{1}=60$, Ch side junction & 3.01 & $0.90 \cdot 10^{5}$ \\
$\mathrm{P}_{1}=40$, Co side junction & 3.27 & $0.48 \cdot 10^{5}$ \\
$\mathrm{P}_{1}=50$, Co side junction & 3.13 & $1.04 \cdot 10^{5}$ \\
$\mathrm{P}_{1}=60$, Co side junction & 2.56 & $1.86 \cdot 10^{5}$ \\
Average & 3.08 & $0.80 \cdot 10^{5}$ \\
\hline \hline
\end{tabular}

Table 4.3, Fitting constants found in different measurements and their average

Like was done for the average heat transfer, the energy transferred from the gas to the reactor walls and piston can be compared to the work done on the gas. In Figure 4.11 the energy lost to the cover and piston is calculated based on a simulated pressure trace of a compression expansion cycle with a peak compression pressure of 120 bar (the simulation was done with the numerical model described in section 5.6 ). The work done on the gas is calculated using the simulated reactor volume and equation 4-5. 


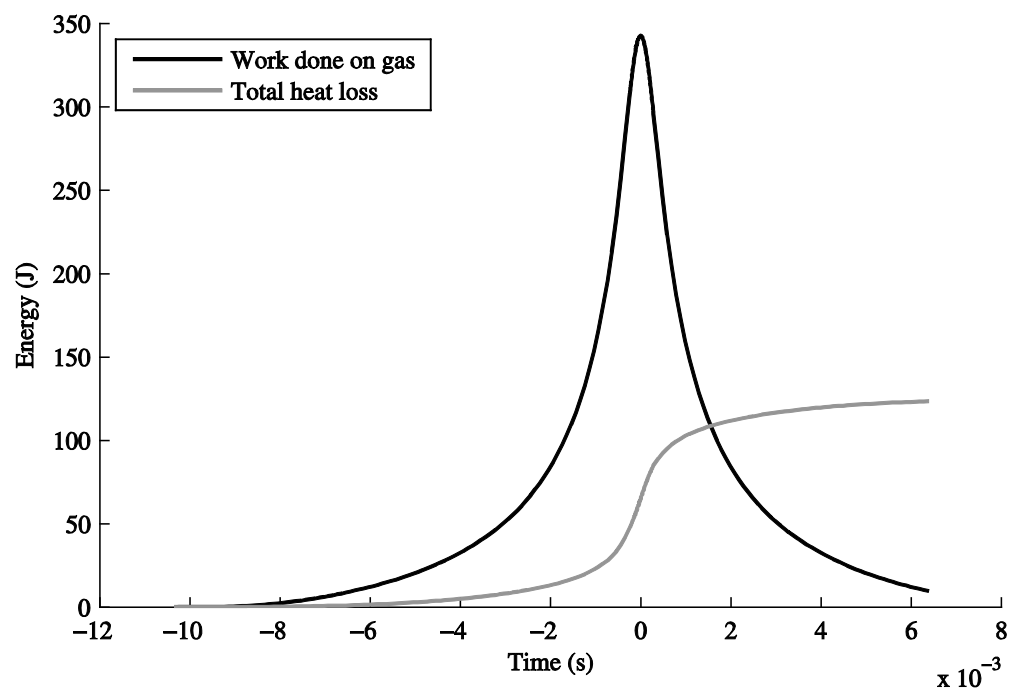

Figure 4.11, Total heat lost compared to work done on gas, peak compression pressure 120 bar

The final total amount of energy lost; during the first compression expansion stroke is then calculated to be $36 \%$ of the maximum work done on the gas. This value can be considered and upper bound for the heat loss, as more reciprocations occur and steady state is approached the heat loss will decrease and may eventually even disappear.

\subsubsection{Comparing instantaneous heat losses to averaged heat transfer}

The results obtained in the average heat loss measurements cannot be directly compared to the instantaneous heat loss because they were determined in different reactors. The stroke length, piston size and mass and cover geometry are different for the two reactors, which influences the found results. However, the order of magnitude of the losses found can be compared, and they are similar. The instantaneous heat losses between 0 and 20 bar vary between 0 and $1.6 \cdot 10^{6} \mathrm{Wm}^{-2}$. The average heat loss when peak pressure is 20 is found to be $2.4 \cdot 10^{4} \mathrm{Wm}^{-2}$. The fact that there is two orders of magnitude difference between the instantaneous heat loss and average heat loss is perfectly acceptable, since the peak pressures only occur during very short periods in the operation of the PCR, making the average heat loss much lower than the instantaneous heat loss. At the same time, the highest average heat loss measured was after between 50 and $400 \mathrm{~s}$, while the instantaneous heat flux is measured during the first reciprocation. 


\section{Syngas from methane with the PCR}

One of the main research goals of project that this thesis is part of is the production of synthesis gas from methane with the PCR. Within this thesis, some of the fundamentals of syngas production from methane with the PCR are investigated. This is done by means of single shot experiments, investigating single isolated parameters, and modeling of the obtained results.

\subsection{Introduction}

The PCR can be used for a multitude of applications, including endothermic and exothermic gas phase reactions, heterogeneous gas-liquid reactions and even solid-gas reactions. Within the scope of this project, the production of syngas from methane is investigated. This is done using the single shot reactor that was described in section 0 . The goal of the experiments is to isolate several parameters, and investigate their influence on the syngas yield. These parameters can be summed up as follows and will further be explained in the following sections.

- Reactant composition

- Initial reactor and reactant temperature

- Piston kinetic energy

- Piston mass

- Cover geometry

\section{Reactant composition}

To investigate the influence of reactant composition on the product, experiments are performed with different reaction mixtures. In Table 5.1 the reaction mixtures that are used for the partial oxidation experiments are shown.

\begin{tabular}{lllll}
\hline \hline Mixture & $\mathrm{CH}_{4}$ & $\mathrm{O}_{2}$ & $\mathrm{~N}_{2}$ & $\mathrm{Ar}$ \\
\hline 1 & 8.8 & 4.9 & 19.0 & 67.3 \\
2 & 8.8 & 3.8 & 20.4 & 67.0 \\
3 & 9.6 & 5.3 & 85.1 & - \\
\hline \hline
\end{tabular}

Table 5.1, Compositions of mixtures used in the partial oxidation experiments 
These mixtures were chosen to be close to the stoichiometric composition for syngas production by partial oxidation. Argon was used as a dilutant to increase compression temperatures and for safety reasons. Mixture 1 contained a little more oxygen than required stoichiometrically, mixture 2 a little less. Mixture 3 did not contain argon. Instead nitrogen was used for dilution, in order to provide information on the net effect of the presence of argon.

In Table 5.2 the composition mixture used in the steam reforming experiments is shown. Mixture 4 is a mixture where steam and methane are present in their soichiometric ratio of $1: 1$.

\begin{tabular}{llll}
\hline \hline Mixture & $\mathrm{CH}_{4}$ & $\mathrm{H}_{2} \mathrm{O}$ & $\mathrm{Ar}$ \\
\hline 4 & 6.7 & 6.7 & 86.6 \\
\hline \hline
\end{tabular}

Table 5.2, Composition of mixture used in the steam reforming experiments

\section{Initial reactor and reactant temperature}

The reactor was preheated to three different temperatures to take a look at the reactor performance with preheated reactant streams. The three temperatures were $295 \mathrm{~K}$ (which was the room temperature), $423 \mathrm{~K}$ and $543 \mathrm{~K}$. Reactants were allowed to remain in the reactor for 5 minutes after filling the reactor with the reactants, to ensure that the temperature of the reactants was uniform and the same as the reactor temperature. Partial oxidation experiments were performed at the three different temperatures with the three different reactant compositions, with the exception of mixture 3 , which was only used at the two elevated temperatures. The reason for this is that without argon dilution the compression temperatures were not high enough for substantial conversions. Steam reforming experiments were performed at the two elevated initial temperatures only, since the steam in the mixture would condense at room temperature.

\section{Piston kinetic energy}

Since the kinetic energy of the piston may change during the operation of the continuous $\mathrm{PCR}$, and can only be controlled indirectly by changing external parameters, it is important to know the relation between piston kinetic energy and product composition. By using different launch pressures in the launch mechanism, different piston kinetic energies could be obtained. For each combination of initial temperature, chemical composition of reactants and piston type a series of measurements is done with different maximum piston kinetic energies. 


\section{Piston mass}

One of the ways to tune the pressure history of the process is by changing the density of the piston used. To investigate the influence of the piston mass on the reaction products, two different types of pistons are used. The first piston was made of graphite (Poco, ACF10Q) and the second of metal impregnated graphite (Toyo Tanso, GM-5). The mass of the graphite piston was $0.854 \mathrm{~kg}$, and the mass of the metal impregnated graphite $3.16 \mathrm{~kg}$.

\section{Cover geometry}

In order to see whether the effect that the walls have on the chemistry can be influenced by the geometry of the cover, different cover geometries have been designed. These have been introduced in section 0 . For each of the cover geometries, a series was performed using mixture 1 , the lighter of the two pistons and at the three initial temperatures.

\subsection{Single shot reactor performance}

\subsubsection{Single shot behavior}

One of the key aspects in investigating the chemistry of the PCR is obtaining true single shot behavior. This means that only during one compression expansion cycle chemical reactions take place and that further reciprocations are significantly damped. If this is achieved, the effect of one compression expansion cycle can be isolated. In Figure 5.1 the pressure trace of an experiment with chemical reactions is shown. In the pressure trace several reciprocations can be seen, where the second reciprocation pressure is approximately half of the pressure during the first reciprocation. This means that, as long as the compression during the first stroke is not more than twice than what is needed for substantial chemical transformations to take place, there will not be chemical reactions during further reciprocations. 


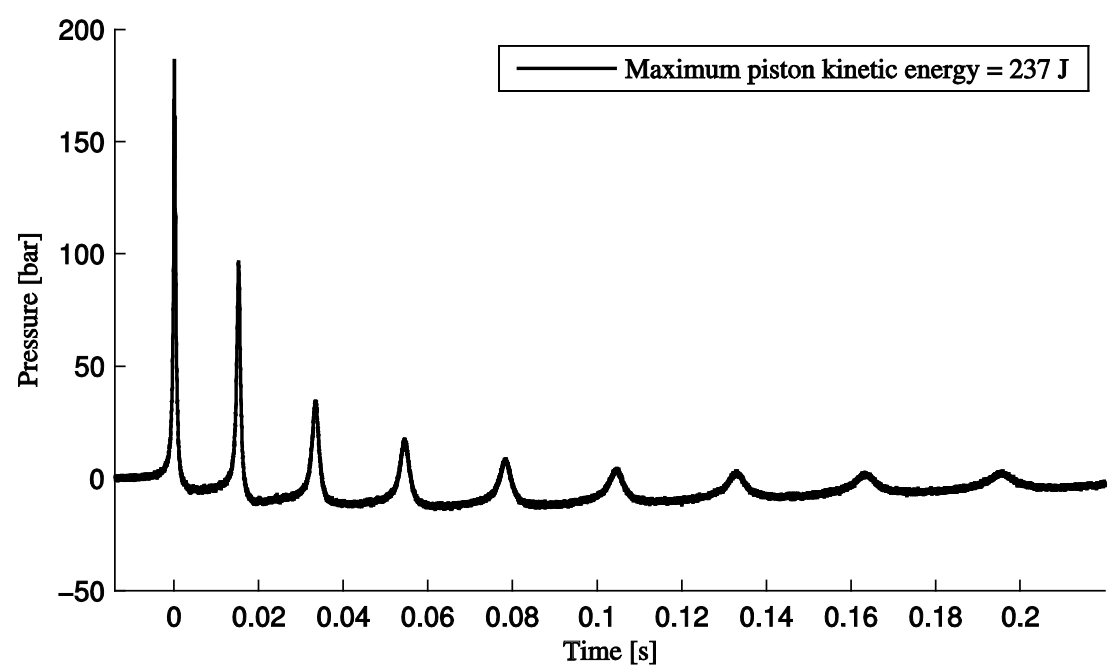

Figure 5.1, Pressure trace of experiment with chemical reactions, maximum piston kinetic energy is $237 \mathrm{~J}$

\subsubsection{Velocity sensor results}

In Figure 5.2 the achieved maximum piston velocity as a function of the used launch pressure is plotted. There is, especially for the high launch pressure - high velocity region quite a bit of spread in terms of achieved velocity. For example, for the experiments done with a launch pressure of 110 bar, maximum piston velocities range from 26 to 28 $\mathrm{m} / \mathrm{s}$. This spread can be attributed to one of two factors: either the velocity sensor accuracy is low, or the same launch pressure does not always result in the same maximum piston velocity (or a combination of both). Both factors can be argued for. The higher inaccuracy of the velocity sensors for higher velocities can be explained by the working of the sensor: as piston velocities become higher, the grooves in the piston become harder to distinguish. On the other hand, higher launch pressures mean higher forces on the piston, thus also (possibly) higher shear forces and so a larger spread in final maximum piston velocities.

In practice, as will be seen in section 5.3 , using the piston velocity as a parameter by which to compare various experimental results turned out to be a very reliable and robust method. This would lead one to conclude that the source of noise in the launch pressure - maximum piston velocity relation is mainly to be attributed to the maximum piston velocity actually being different for the two experiments with the same launch pressure. 


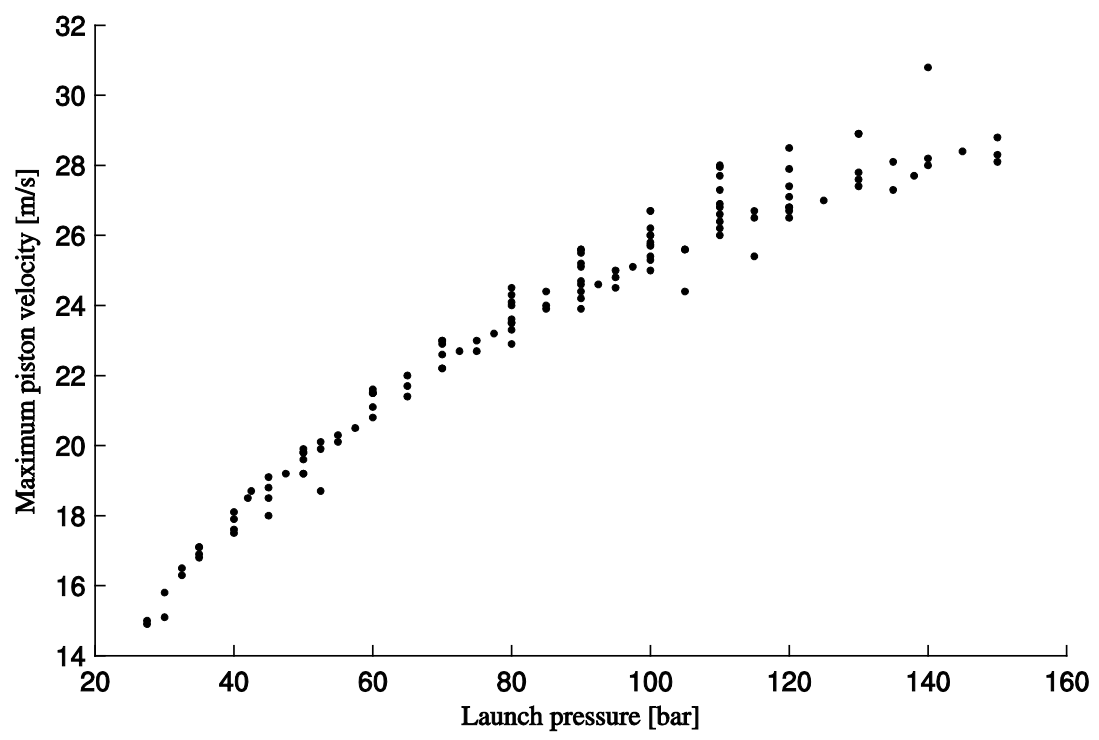

Figure 5.2, Maximum piston velocity as a function of launch pressure for the experiments performed with the $860 \mathrm{~g}$ piston

\subsection{Experimental results}

In the following section the results of the experiments are shown. In order to make the results comparable the yields of products are plotted as a function of the maximum piston kinetic energy. The reason for this is that this best describes the "input" into the system: the piston kinetic energy is a measure for the energy that is supplied to the reaction mixture. So, if experiment and simulation have the same energy input, their output in terms of yield can be compared. 


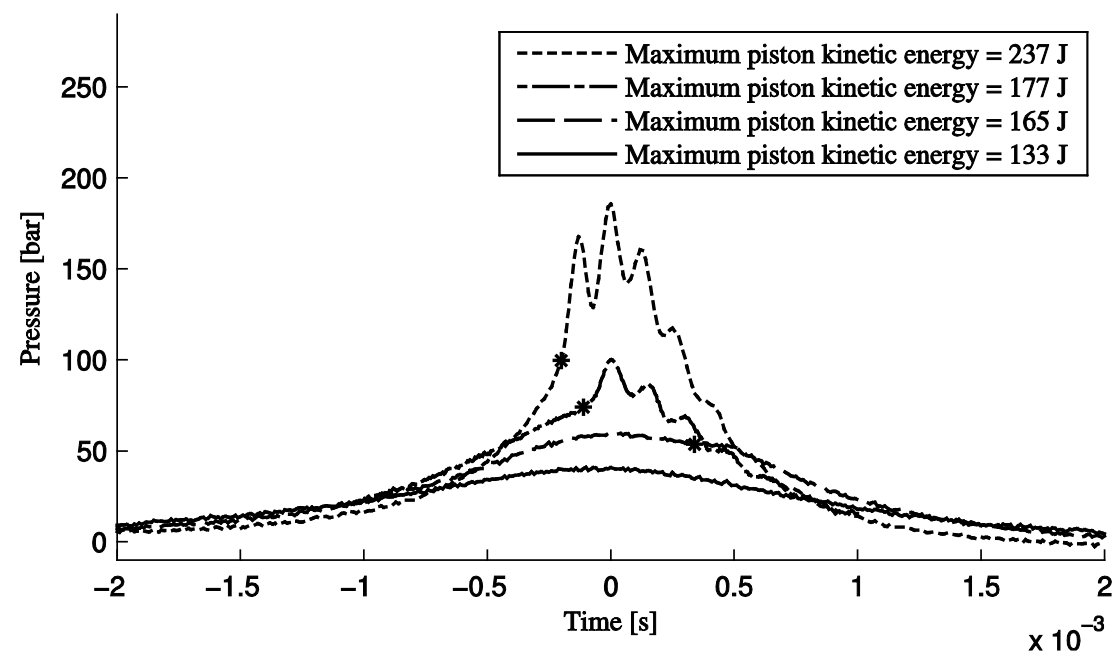

Figure 5.3, Pressure traces of four experiments with mixture 1 with different maximum piston kinetic energy

In Figure 5.3 the pressure traces of four experiments are shown. The experiments were performed in a preheated reactor at $423 \mathrm{~K}$ using mixture 1 . The curves are shifted in time such that the highest pressure is at $t=0$. For the lowest energy input in this series (133 J) no ignition of the mixture in the reactor takes place. The pressure curve simply shows a smooth compression expansion cycle, without any humps caused by ignition. As the energy input is increased, the measurement with the maximum piston kinetic energy of $165 \mathrm{~J}$ shows ignition just after the piston reached top dead centre (TDC), indicated by an asterisk in the graph. As the energy input is increased stepwise again and again, the ignition moment moves forward relative to the cycle, igniting around and even before the TDC. The oscillating pressure signal after the initial ignition moment can most likely be attributed to the characteristics of the pressure sensor, its mounting method and possibly reaction kinetics, but the exact cause is still unsure.

\subsection{Partial oxidation results}

\subsubsection{Hydrogen yield}

In Figure 5.4 the hydrogen yield as a function of maximum piston kinetic energy is shown for the three different initial temperatures used, for mixture 1 . The yield is expressed as a fraction of the theoretical maximum yield, as introduced in section 3.3.7 (so a yield value of 1 means than an equal number of hydrogen atoms that were originally in the methane are now in the form of hydrogen, a yield value of 0 means all the hydrogen 
atoms are bound in other compounds than hydrogen). When looking at the data, the trend that was observed in the different pressure traces in Figure 5.3 is visible in the hydrogen yield also. For low kinetic energies (what constitutes low is different for the different initial temperatures), no significant reaction takes place, and no hydrogen is produced. Then, as the energy input is increased, at a well defined threshold value the hydrogen yield suddenly goes up, as ignition events become possible. Higher energy inputs cause the hydrogen yield to climb further still. The hydrogen yield just beyond the threshold is about $30 \%$, while the maximum yield recorded is $83 \%$.

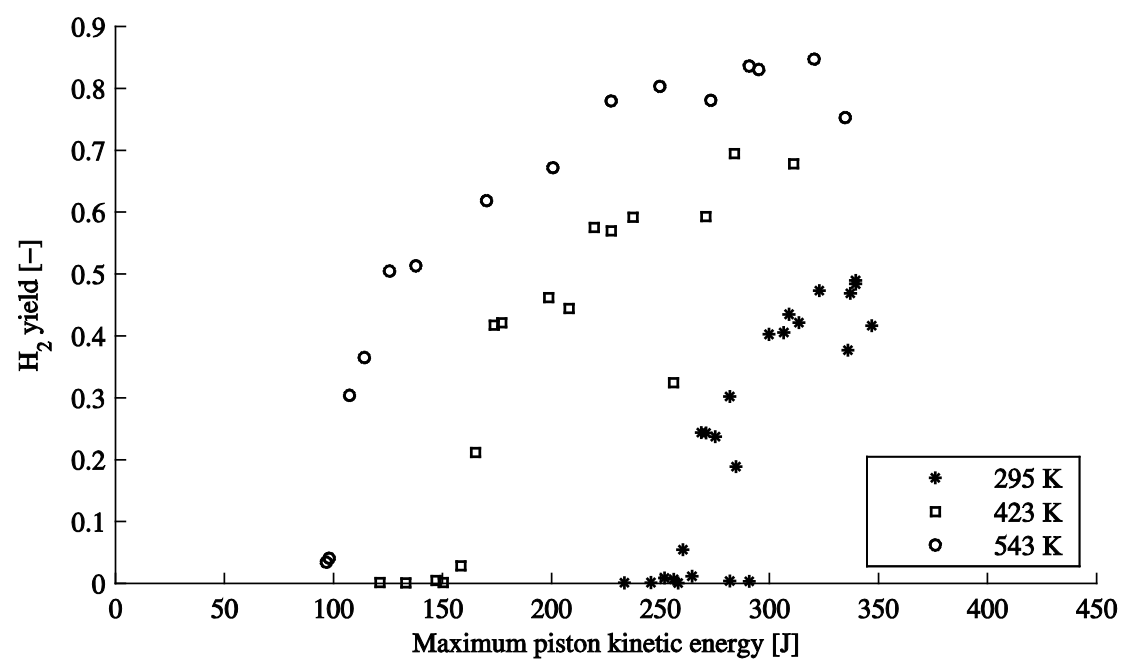

Figure 5.4, Hydrogen yield of experiments with mixture 1, with different initial temperatures

When comparing the different initial temperatures, it is visible that an initial temperature change merely shifts the entire hydrogen yield graph along the piston kinetic energy axis, without having much effect on the shape of the graph. The threshold energy input for significant reactions to take place becomes lower, as initial temperature is increased. For the mixture used in these experiments, the threshold energy input for significant reactions to occur with an initial temperature of $295 \mathrm{~K}$ corresponds to a maximum piston kinetic energy of about $260 \mathrm{~J}$. The threshold energy input for significant reactions to occur with an initial temperature of $543 \mathrm{~K}$ corresponds to a maximum piston kinetic energy of about $100 \mathrm{~J}$. 


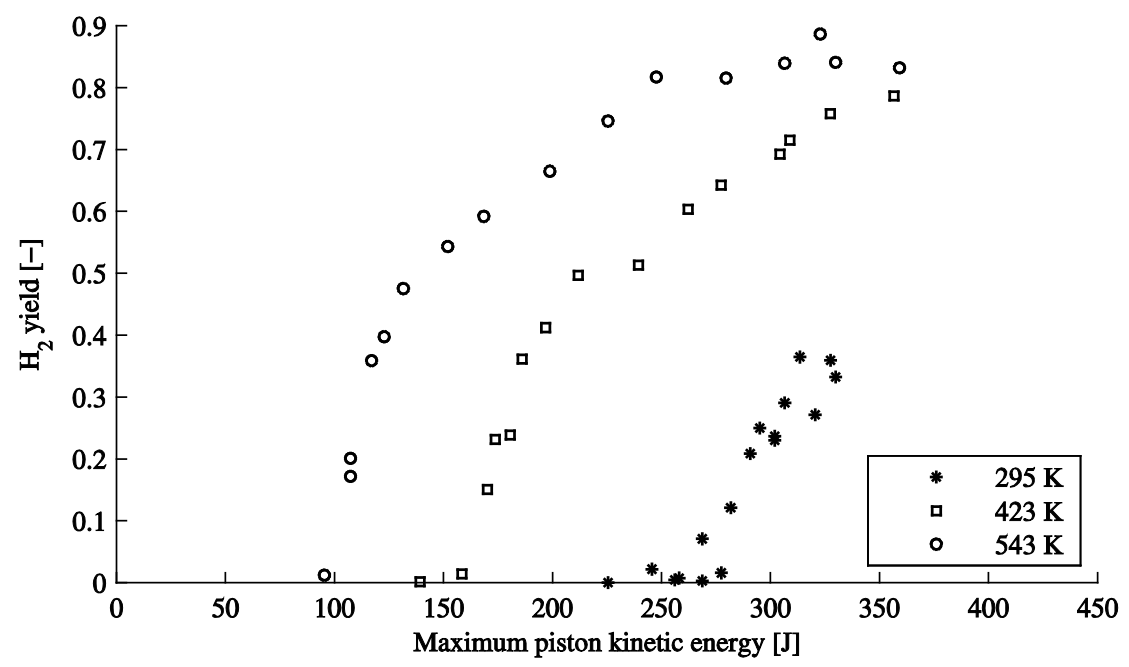

Figure 5.5, Hydrogen yield of experiments with mixture 2, with different initial temperatures

In Figure 5.5 the yield of hydrogen is shown for mixture 2 and in Figure 5.6 the yield for mixture 3. As mentioned, mixture 3 was only used for experiments with elevated initial temperatures. The graph that is obtained with mixture 2 closely resembles the result that was obtained with mixture 1 . This indicates that the oxygen concentration does not influence the hydrogen yield noticeably.

The result obtained with mixture 3 on the other hand, does differ significantly from the other two mixes. While the shape of the graph is still the same, the energy threshold for significant reactions is a lot higher. The energy threshold for the $543 \mathrm{~K}$ initial temperature is around $150 \mathrm{~J}$ for mixture 3 . This can be explained by the absence of argon in this mixture. The adiabatic compression constant of argon is higher than that of nitrogen, so the temperature of mixture 3 will be lower for the same compression energy. This means that the energy of the reciprocating piston should be higher to have the same conversion. 


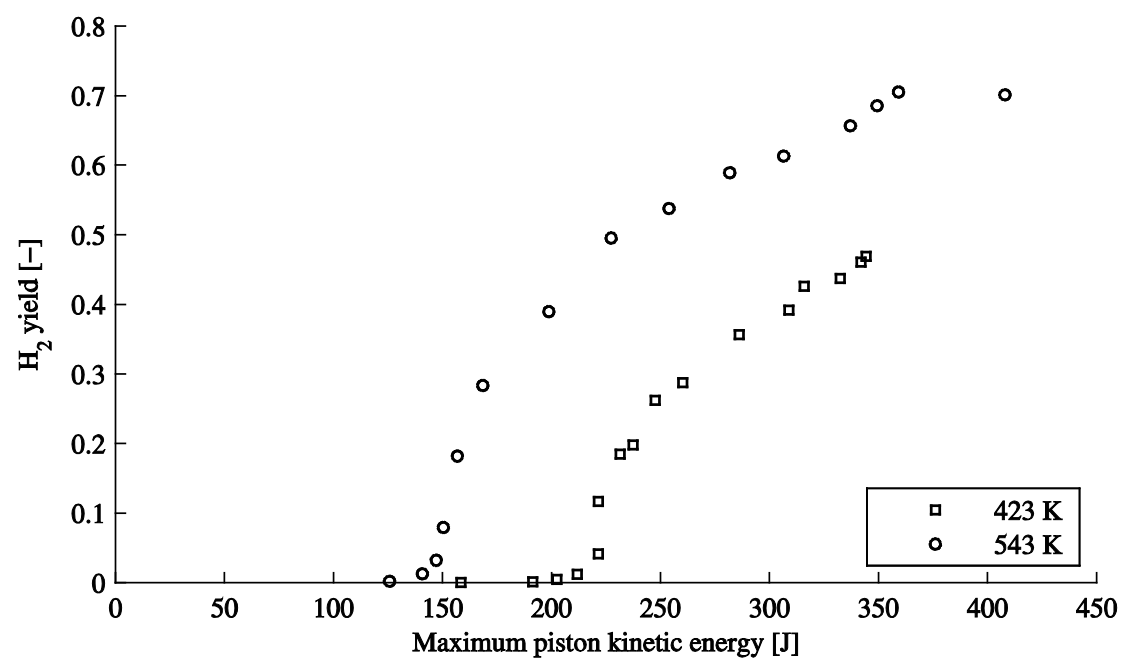

Figure 5.6, Hydrogen yield of experiments with mixture 3, with different initial temperatures

\subsubsection{Carbon monoxide yield}

In Figure 5.7 the yield of carbon monoxide as a function of the maximum piston kinetic energy is plotted. The yield is defined the same way as the previously discussed hydrogen yield. A striking resemblance is seen to the hydrogen yield results. Clearly, both hydrogen and carbon monoxide are produced at the same moment. The same as with the hydrogen results, the carbon monoxide yield just beyond the threshold is relatively low, only about $40 \%$. The carbon monoxide production increases as piston kinetic energies are increased further beyond the threshold, to a maximum recorded yield of $87 \%$.

In Figure 5.8 the yield of carbon monoxide is shown for mixture 2 and in Figure 5.9 the yield for mixture 3 . In contrast to the results on hydrogen formation, there is a significant difference between the carbon monoxide yield curves obtained with mixtures 1 and 2 . There is a difference both in maximum yield, as well as in the shape of the piston kinetic energy-yield curve. The yield of carbon monoxide from mixture 2 is lower than the yield of mixture 1 (a maximum yield of .74 from mixture 2 compared to a maximum yield of .88 from mixture 1 ). This indicates that while the oxygen concentration does not influence the hydrogen yield noticeably, it does influence the yield of carbon monoxide: less oxygen in the reactants mean less carbon monoxide in the products. 


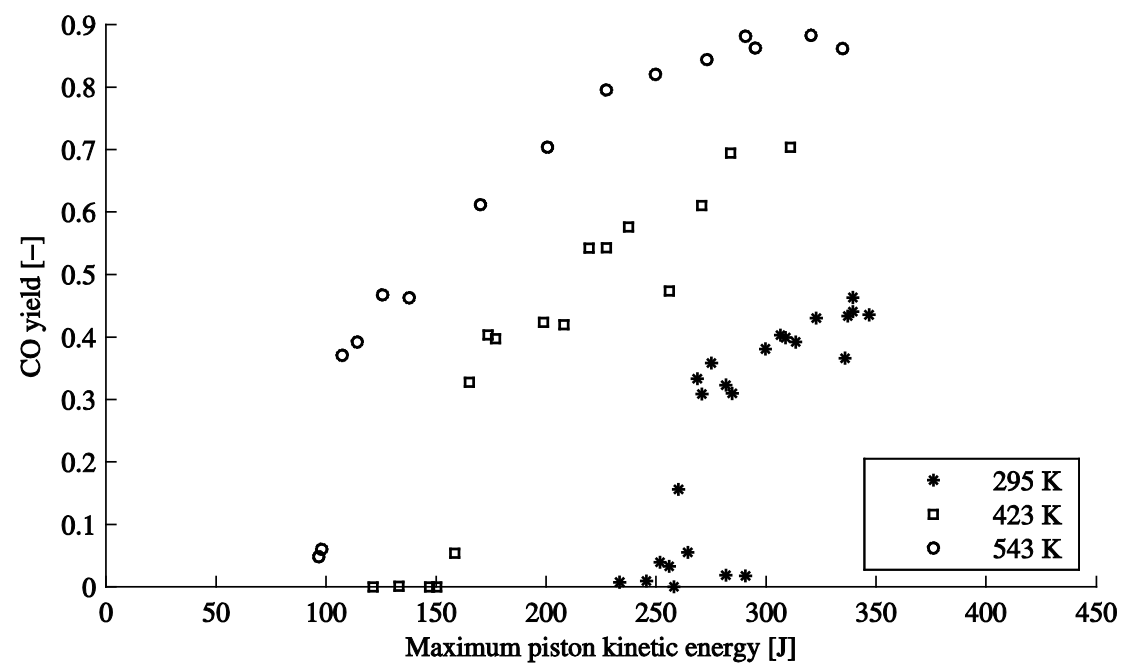

Figure 5.7, Carbon monoxide yield of experiments with mixture 1, with different initial temperatures

The shape of the piston kinetic energy-yield curve obtained from mixture 1 shows a smooth transition from the very steep gradient of the yield at the threshold energy, to the more gradual increase of the yield beyond the threshold. The yield curve from mixture 2 on the other hand has a sharp angle between the initial jump at the threshold energy, and a more horizontal shape just beyond the threshold. This would lead to the conclusion that the process can be divided into at least two stages. The first stage being almost instantaneous at the threshold and combustion like of character. The second stage appears to be endothermic, requiring more energy than that threshold to be initiated. This second stage then only proceeds when more energy is supplied than the threshold. In mixture 1 the amount of energy that is released is larger in the first stage (since more oxygen in present), so the subsequent endothermic stage happens sooner. In mixture 2 less oxygen is present, thus less energy released during the first, combustion like, stage thus more energy needs to be supplied to the piston to initiate the second endothermic stage (leading to a more horizontal shape just beyond the threshold). 


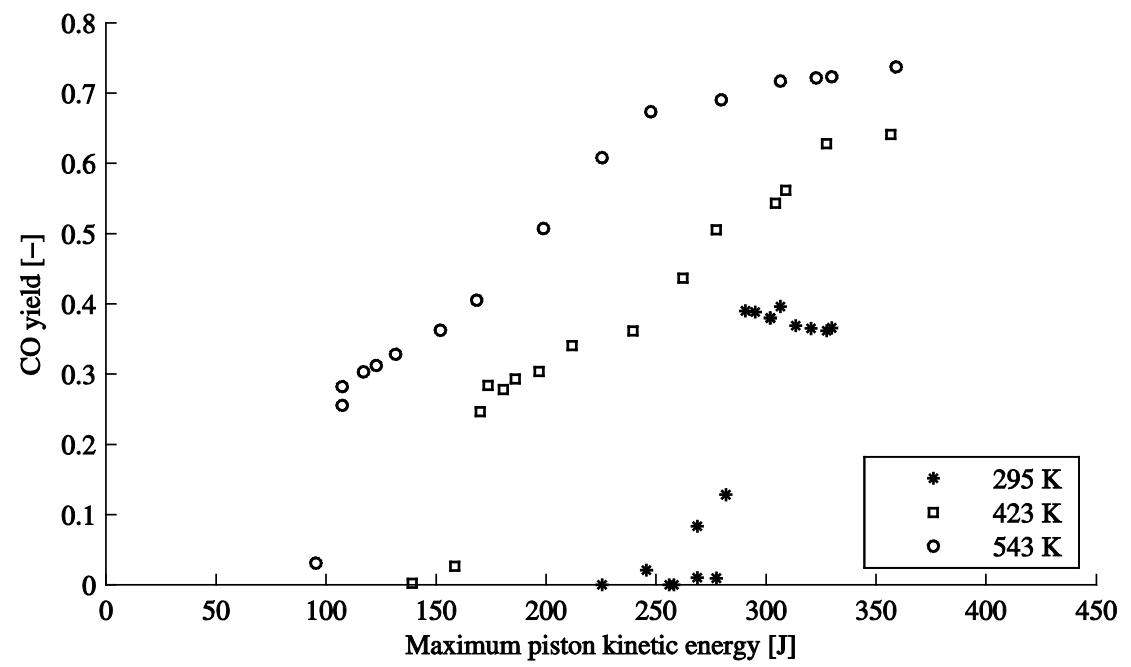

Figure 5.8, Carbon monoxide yield of experiments with mixture 2, with different initial temperatures

The result obtained with mixture 3 lies somewhere between the results of mixtures 1 and 2 . There is some angle visible between the initial jump and further increase, but not as pronounced as in the mixture 2 result. As with the hydrogen yield, the curve is shifted along the energy axis when compared to mixtures 1 and 2 .

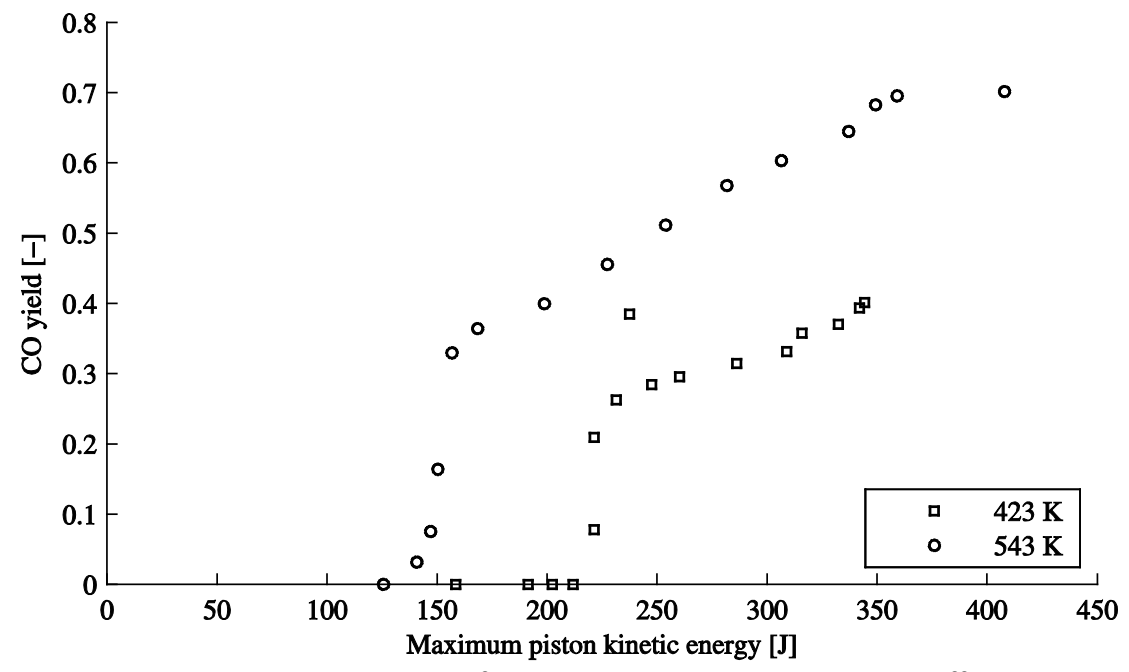

Figure 5.9, Carbon monoxide yield of experiments with mixture 3 , with different initial temperatures 


\subsubsection{Oxygen concentration in product gas}

An important quality that the produced syngas must possess is that it contains no oxygen. So, the concentration of oxygen in the products of the experiments performed is an aspect that must be considered.

In order to clearly pin point the moment of disappearance of oxygen, the yield is plotted not as a function of the maximum piston kinetic energy, but as a function of the methane conversion (determined according to equation 3-25). In this way, it becomes clear whether the specie under investigation is formed or consumed in the beginning of the conversion process (when the reaction is quenched quickly, and little methane has been converted), or near the end on the conversion process (when the reaction is allowed to proceed further, and more methane is converted). An added advantage of this method of plotting is that all eight experiments (with different initial mixes and initial temperatures) can be compared together in one graph.

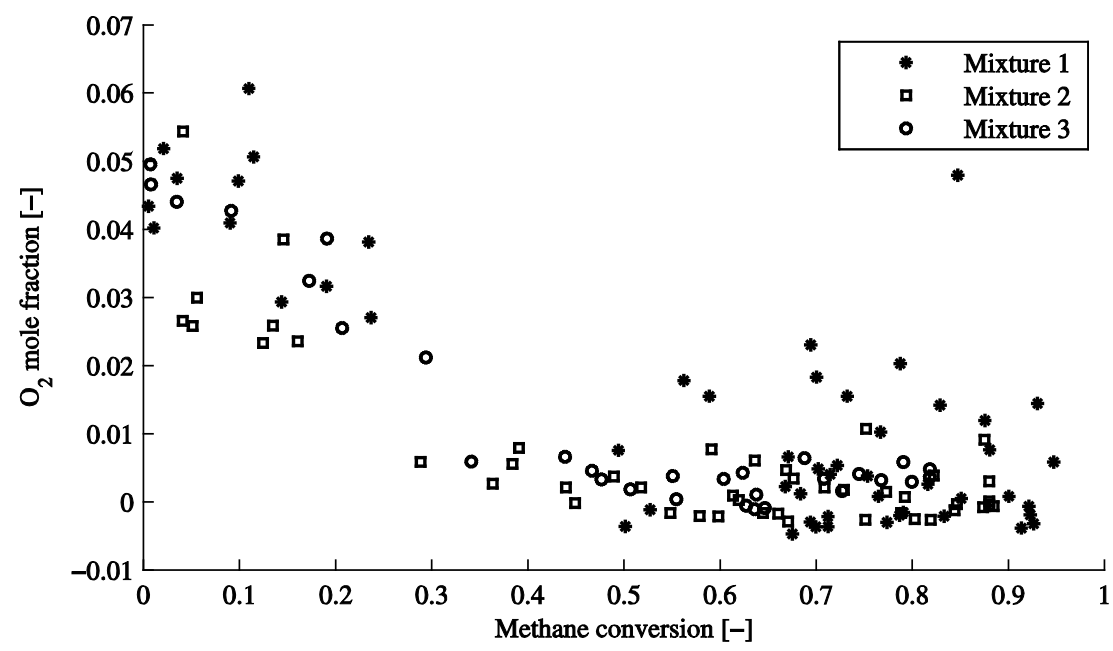

Figure 5.10, Oxygen concentration in the product of the experiments with different initial compositions and different initial temperatures

In Figure 5.10 the oxygen concentration found in the product of the experiment is shown as a function of the methane conversion. While the concentration of oxygen is close to the initial concentration of the mixtures when little methane has converted (below 20\% conversion), the concentration of oxygen quickly goes to zero above $30 \%$ methane conversion. There are a few measurements that shown a significant concentration of oxygen (between 1 and 2\%) even in the high methane conversion range, but these can most likely be attributed to the air that is enclosed at the connecting and disconnecting 
of the sample bag. The error that this procedure introduces was discussed in more detail in section 3.3.7. The effect of this error is also visible in the data that is yielded in the very low conversion range. When no methane is converted, it means no reaction has taken place at all, so one would expect the oxygen concentrations to be equal to the concentration in the original reactant mixture. However, concentrations are higher than the concentrations in the original mixture, showing the effect of the air enclosed in the sample bag during the experimental procedure.

The almost zero concentration in the product of all mixtures leads to the conclusion that the initial jump in product yields is indeed a combustion like step, in which all oxygen present in the reaction mixture is consumed.

\subsubsection{Minor species by partial oxidation}

\section{Carbon dioxide}

In Figure 5.11 to the yield of carbon dioxide of the different experimental series is shown. The top graph in Figure 5.11 shown the yield obtained with mixture 1, the middle graph mixture 2 and the bottom graph mixture 3 . The first observation that can be made is that the carbon dioxide yield depends on the kinetic energy in a much different way than the hydrogen and carbon dioxide. While the hydrogen and carbon monoxide yields are the highest with the highest piston kinetic energy, the carbon dioxide yield is highest just after the threshold kinetic energy, and decreases for higher energies. This indicates that the carbon dioxide is formed during the combustion-like step in the beginning of the chemical conversion process, and is then used up again if the process remains unquenched longer. An important conclusion that can be drawn from this is that carbon dioxide reforming of methane is also feasible in the PCR.

As is with carbon monoxide, a dependence on the initial oxygen concentration is visible. In the case of carbon dioxide this dependence is stronger: the maximum carbon dioxide yield obtained with mixture 1 is .048 , while the maximum yield obtained with mixture 2 is .025 . 

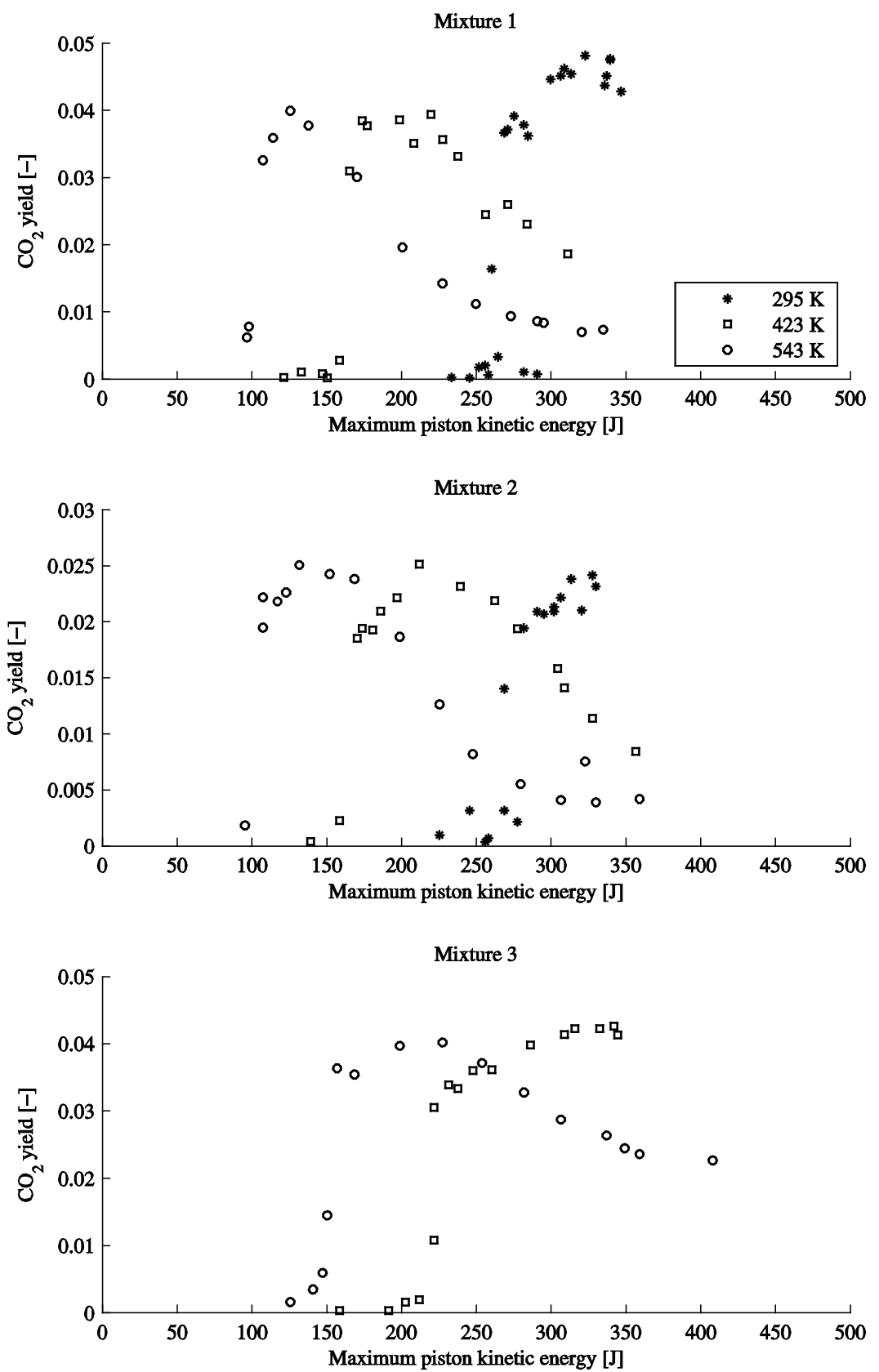

Figure 5.11, Carbon dioxide yield of experiments with mixture 1 (top), 2 (middle) and 3 (bottom), different initial temperatures 


\section{Acetylene, ethylene and ethane}

In Figure 5.12 the yield of the three C-2 side products are plotted as a function of methane conversion. There is a clear order visible, for the formation of C-2 side products. Ethane is formed first, when the reaction has not proceeded far. The highest ethane yields are found when between 10 and $30 \%$ of the methane has been converted. After this, ethylene is formed, with highest yields when between 30 and $80 \%$ of the methane has converted. Lastly, acetylene is formed, with the highest yields when the conversion of methane is around $80 \%$. This same trend is visible for all experiments, irrespective of initial temperature and reactant composition.

The order of formation corresponds to the well known Kassel mechanism [63]:

$$
2 \mathrm{CH}_{4} \rightarrow \mathrm{C}_{2} \mathrm{H}_{6}+\mathrm{H}_{2} \rightarrow \mathrm{C}_{2} \mathrm{H}_{4}+2 \mathrm{H}_{2} \rightarrow \mathrm{C}_{2} \mathrm{H}_{2}+3 \mathrm{H}_{2} \rightarrow 2 \mathrm{C}_{(s)}+4 \mathrm{H}_{2} \text {. 5-1 }
$$

This indicates that, besides partial oxidation occurring, methane is also cracked during the compression expansion cycle. 

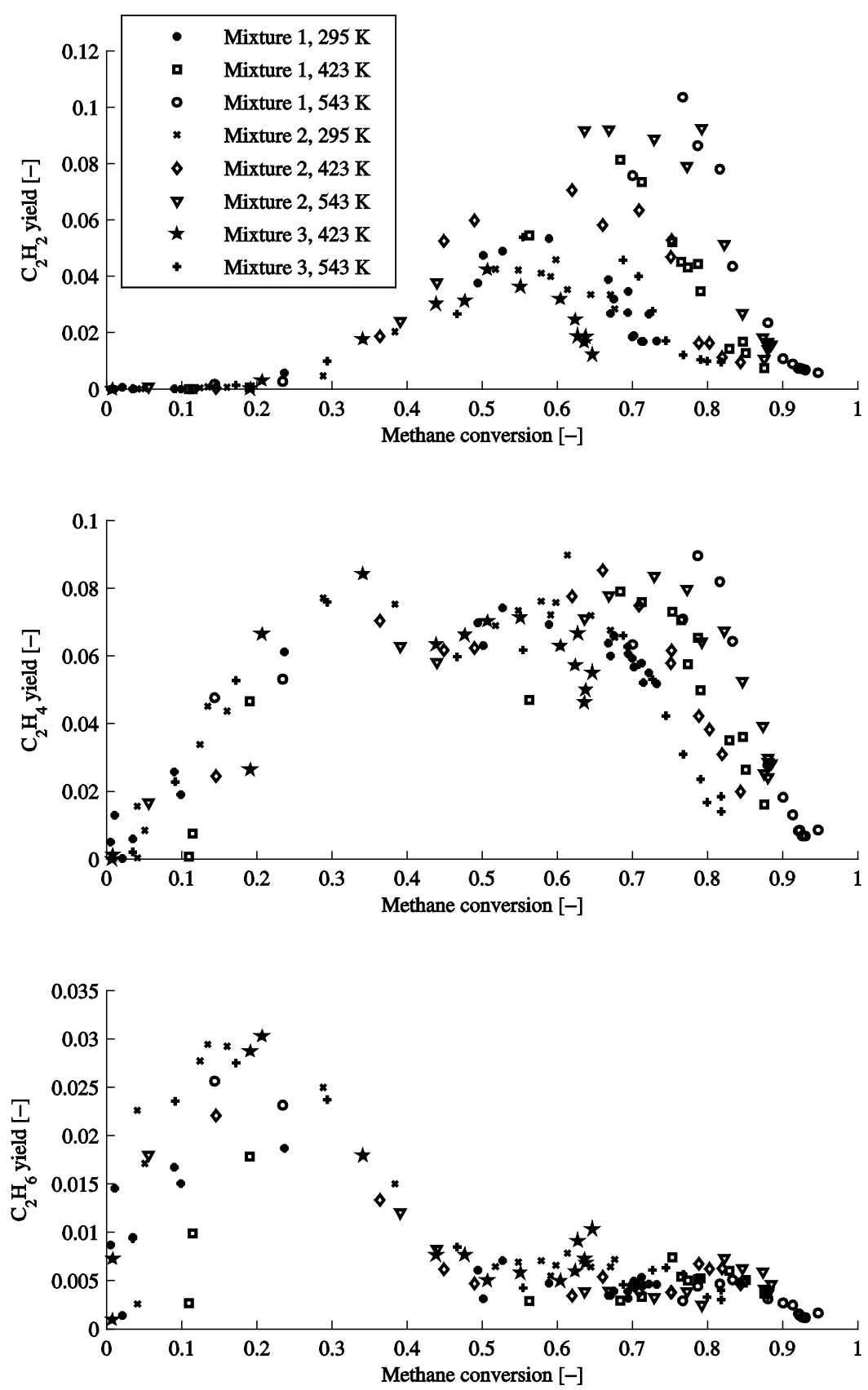

Figure 5.12, Yield of acetylene (top), ethylene (middle) and ethane (bottom), with different mixtures, at different initial temperatures 

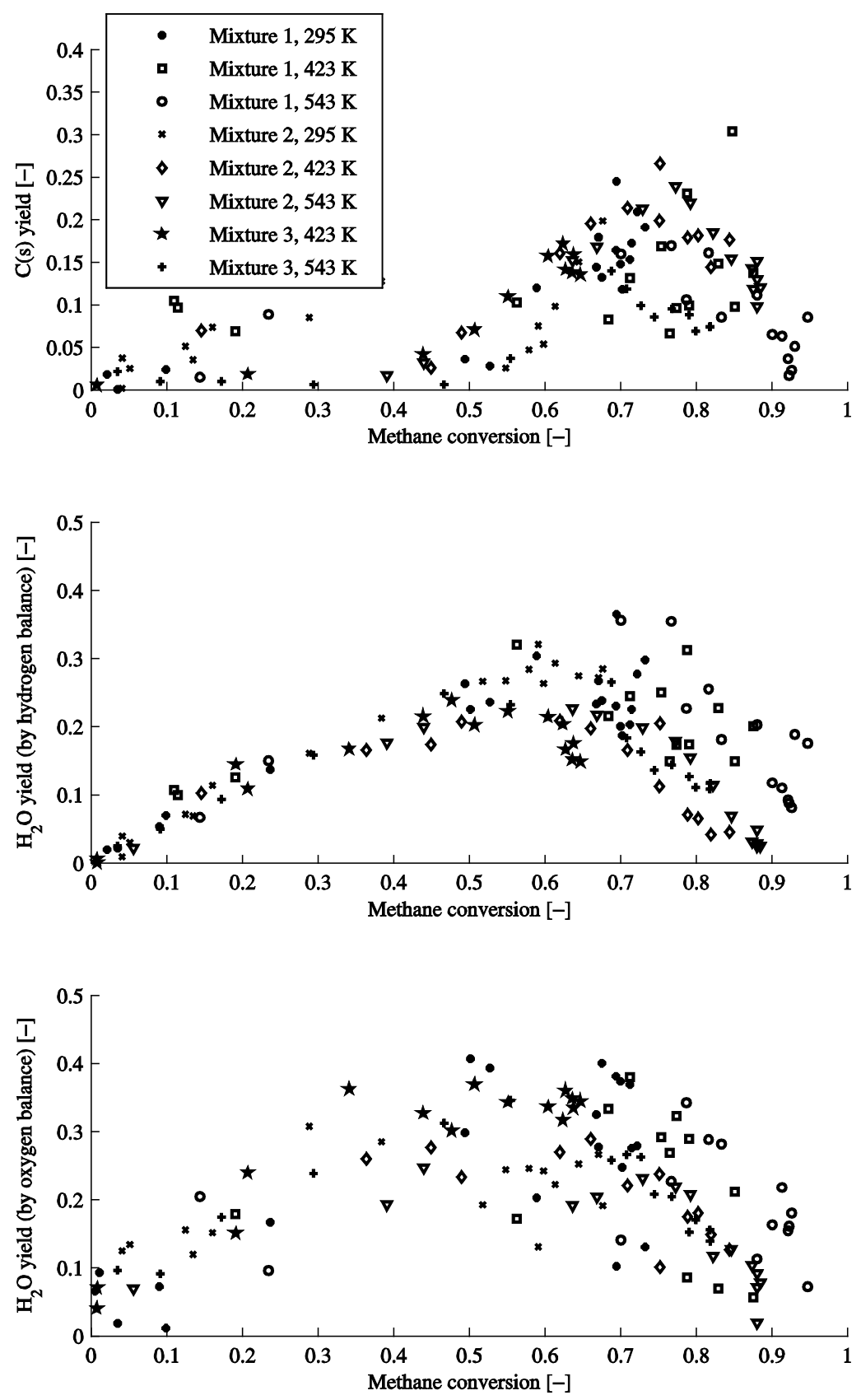

Figure 5.13, Water and soot yield, calculated from the carbon, hydrogen and oxygen balance 


\section{Carbon, hydrogen and oxygen balance}

The gas chromatograph is capable of measuring concentrations of quite a few species, but there are some products formed in the experiments that cannot be measured by GC (at least not by the one that was used in this project). These include water vapor and soot. At the same time it is expected that there may be significant concentrations of water vapor and soot in the product gas. The concentrations of these products can be calculated from the carbon, hydrogen and oxygen balance as shown in Figure 5.13.

The carbon yield is highest for methane conversion levels of between 65 and $80 \%$. There is quite a large spread in the data, but this can be explained by the way that the soot production is calculated: a summation of measured gaseous species concentrations introduces a summation of the errors with which these concentrations were measured. Nevertheless the soot formation appears to drop back down in the direction of zero, near complete methane conversion.

The two ways of calculating the water yielded in the process give a uniform answer. While different methods of calculation are used, the obtained water yield is almost identical. The water yield is highest between 50 and $70 \%$ methane conversion and drops, just like soot, to zero near the complete methane conversion.

\subsubsection{Hydrogen - carbon monoxide ratio}

The syngas produced by the PCR is, only an intermediate product in converting methane or natural gas into other products. For further steps in which the syngas is used to produce other chemicals, the ratio of hydrogen to carbon monoxide in the gas produced is very important. In Figure 5.14 the hydrogen - carbon monoxide ratio of the products produced by the different experimental series are shown. The ratio is depicted as a function of methane conversion. The methane conversion axis is limited to a range from $40 \%$ to $100 \%$. The reason for this is that at low methane conversions, the concentrations of hydrogen and carbon monoxide are very low, thus small errors in the measurement of these concentrations result in very large variations of the hydrogen - carbon monoxide ratio. 


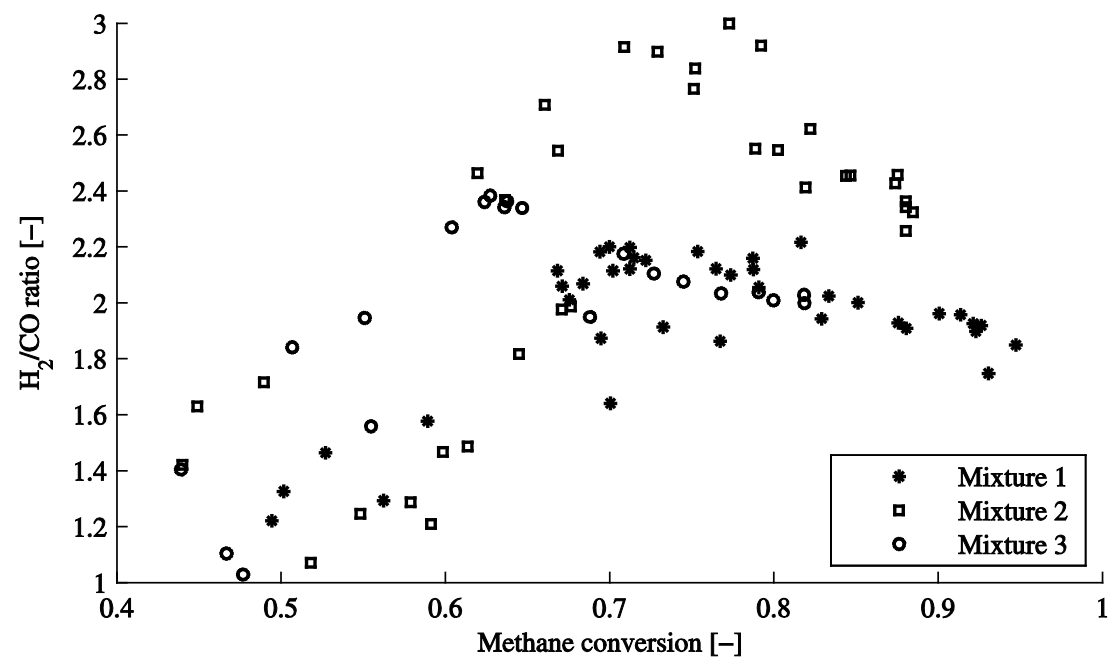

Figure 5.14, Hydrogen - carbon monoxide ratio, with different mixtures, at different temperatures

The shape of the hydrogen - carbon monoxide ratio is comparable for the different mixtures. The ratio increases uniformly to a maximum around $70 \%$ conversion, after which it slowly decreases again. The ratio curve found for mixture 1 starts at around 1.2 for $50 \%$ methane conversion, peaks at around 2.2 for $70 \%$ methane conversion, and then decreases again to about 1.8 for full conversion. The ratio curve for mixture 2 starts around the same point (though the spread of found hydrogen - carbon monoxide ratios around the $40-50 \%$ conversion area is very large) and increases to a maximum of 3 around $75 \%$ conversion. Mixture 3 is, as usual somewhere between the other two mixtures.

The most important conclusion that can be drawn from this is that the hydrogen carbon monoxide ratio is very tunable, by adjusting the oxygen concentration in the reactant mixture. A higher oxygen concentration in the initial mixture results in more carbon monoxide in the product, thus a lower hydrogen - carbon monoxide ratio. Less oxygen on the other hand decreases the carbon monoxide content, increasing the ratio. The presence of argon in the reactant mixture does not seem to influence the hydrogen - carbon monoxide ratio: the ratio is as would be expected for a mixture with argon.

\subsubsection{Piston mass}

To find the effect that the piston mass has on the reaction products, the experiments performed with mixture 3 are repeated, with a piston made of a different, denser material. While the shape and clearance of the piston are the same as with previous 
experiments, the mass is a factor 3.7 more. This means that the reciprocation frequency is lower, and thus the cycle length is longer. Additionally, the energy transfer from the launch system to the piston is more efficient: a lighter piston will move up more quickly, allowing less build up of pressure underneath the piston, while a heavier piston moves up more slowly, allowing more pressure to build in the lower chamber, thus allowing the launch air to perform more work on the piston. This means that for the same launch pressure, the maximum piston kinetic energy will be higher. This is shown in Figure 5.15. However, since it is the piston kinetic energy that is used as a parameter to compare experiments, experimental results with different pistons can still be compared.

In Figure 5.16 the hydrogen yield of experiments preformed with the piston made of metal bonded graphite, with mixture 3 are shown. In this figure, the experiments that were done with mixture 3, with the lighter graphite piston are also shown. The two experiment series that can be compared, namely the $423 \mathrm{~K}$ series, correspond very well. While the piston mass and maximum velocity are very different, the same conversion is reached when the same kinetic energy is given to the piston. Also, while conversion from ambient temperature was not possible with the lighter of the two pistons, the maximum amount of kinetic energy that the heavier piston can have is much higher, so conversion of mixture 3 from ambient initial temperature was possible.

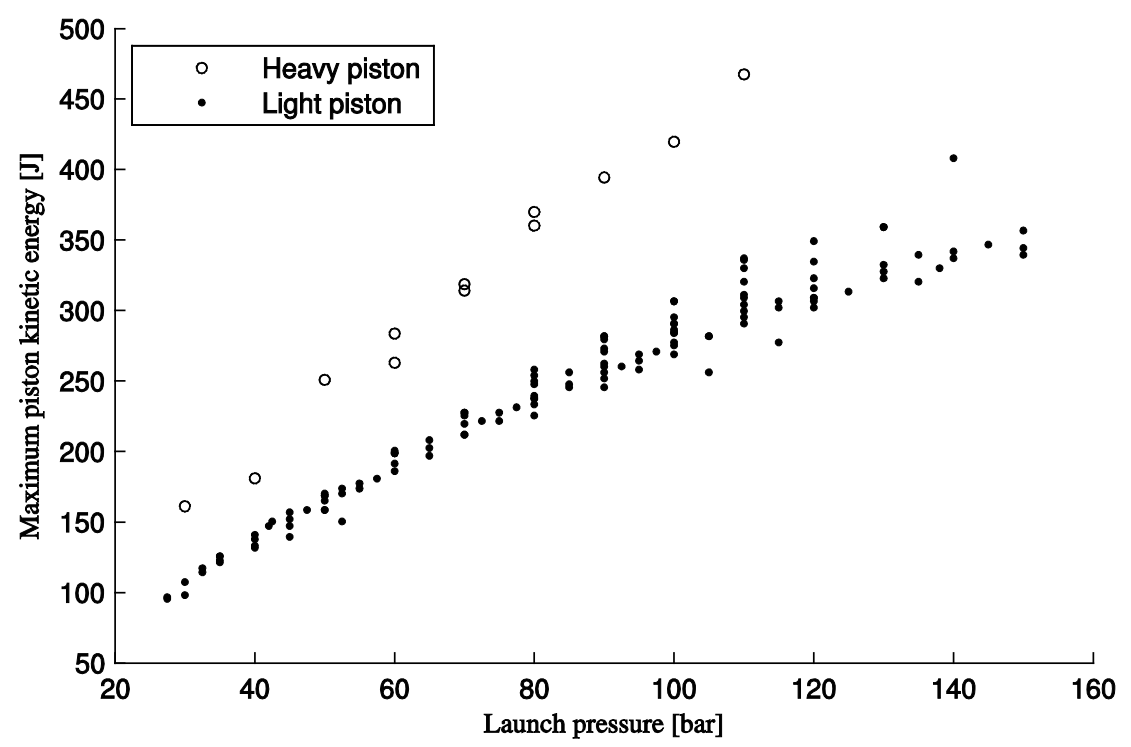

Figure 5.15, Maximum piston kinetic energy as a function of launch pressure, for the two pistons used 
The correspondence between the measurement series with different piston mass means that different piston masses can be used, depending on the requirement of the process. For example, a heavier piston has the distinct advantage that, when used in the single shot reactor, energy can be supplied to the piston much more efficiently. On the other hand, one might prefer to minimize the cycle time of the PCR process, which can be done by reducing the piston mass. This means that the mass of the piston is a powerful design parameter when developing PCR processes.

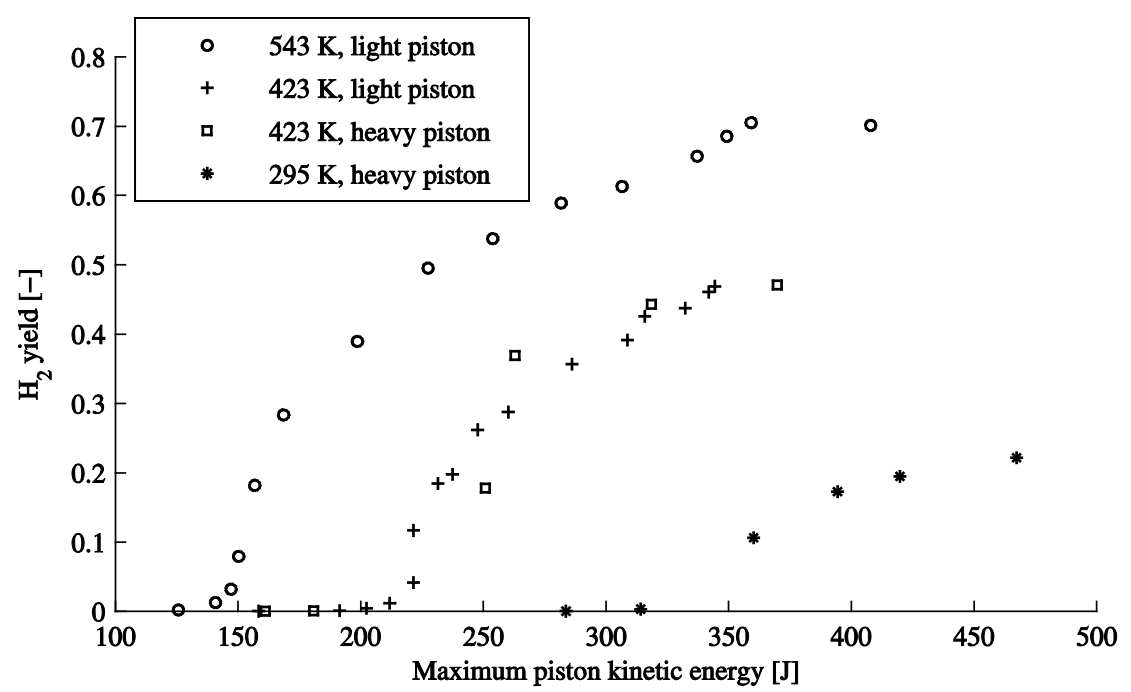

Figure 5.16, Hydrogen yield of experiments with mixture 3 with the heavier piston, with different initial temperatures compared to the lighter piston

\subsubsection{Cover geometry}

Using mixture 1 and the three different initial temperatures, the three alternative cover designs were tested. In Figure 5.17 the hydrogen yield of one of these tests, compared to the normal unmodified flat cover design for the initial temperature $543 \mathrm{~K}$ is shown. In this figure there is no visible difference between the hydrogen yield of the alternative cover and the unmodified cover. The same is true for all of the other experiments with the alternative covers, which are included in appendix III. This would lead one to conclude that either the influence of the walls on the chemistry is so small that a change in wall geometry does not lead to discernable changes in the product, or that the changes applied in the cover design are not large enough to influence the size of the adiabatic core in the reactor. 


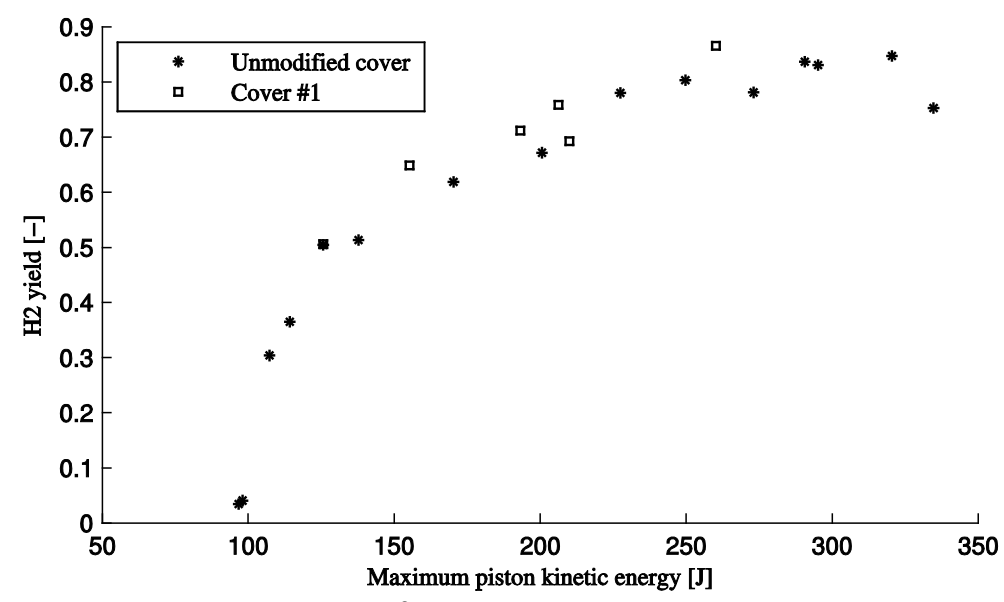

Figure 5.17, Hydrogen yield of experiments with cover 1, with mixture 1 and initial temperature $543 \mathrm{~K}$ compared to the unmodified cover

\subsection{Steam reforming}

An alternative chemical path from methane to syngas is steam reforming. This is an endothermic overall reaction, where methane reacts with steam to form hydrogen and carbon monoxide. The hydrogen yield of a process that is based on steam reforming is theoretically higher, since the steam that is used also contains hydrogen. So, when the same definition of yield is used as is done in the partial oxidation experiments, a yield of more than one can be obtained.

\subsubsection{Hydrogen yield}

In Figure 5.18 the yield of hydrogen of two series of experiments with mixture 4 is shown. The two series are done at initial temperatures of $423 \mathrm{~K}$ and $543 \mathrm{~K}$. Naturally there is no series at ambient initial temperature, since the steam would condense to water.

The shape of the curve that the different measurements form can be divided into two parts. For the $543 \mathrm{~K}$ initial temperature results these two parts roughly stretch from $125 \mathrm{~J}$ maximum piston kinetic energy to $250 \mathrm{~J}$, and from $250 \mathrm{~J}$ to the end of the series. During the first part, a the yield curve flattens off at around $60 \%$ yield. Then it increases more again in the second part, to a maximum yield of around $115 \%$. While the series with initial temperature of $423 \mathrm{~K}$ is likely to contain the same type of division, it is more difficult to see exactly where the two parts begin and end. 


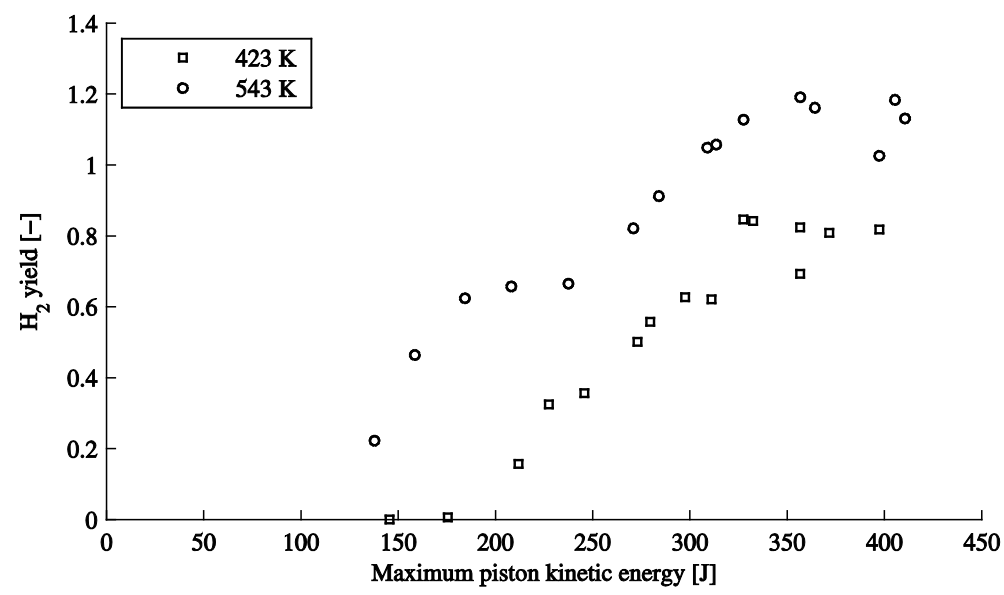

Figure 5.18, Hydrogen yield of experiments with mixture 4 with different temperatures

\subsubsection{Carbon monoxide yield}

The yields of carbon monoxide of the two series done with mixture 4 are shown in Figure 5.19. In this figure the division that was previously identified in the hydrogen yield becomes even more evident. In the $543 \mathrm{~K}$ initial temperature series, at the same energy levels as visible in the hydrogen yields (from $125 \mathrm{~J}$ maximum piston kinetic energy to $250 \mathrm{~J}$ ) hardly any carbon monoxide is formed. In the second part, from $250 \mathrm{~J}$ maximum piston kinetic energy to the end of the series, the yield of carbon monoxide rises to a maximum yield of $35 \%$. It is not clear whether a higher carbon monoxide yield can be obtained with a higher maximum piston kinetic energy.

In the $423 \mathrm{~K}$ initial temperature series, hardly any carbon monoxide is formed at all. Again it is not entirely evident whether the division between the first and second part is around $300 \mathrm{~J}$ maximum piston kinetic energy, or whether all the experiments lie within the first part.

The conclusion that can be drawn is that the first step is a methane cracking step, forming only hydrogen and side products, and that the second step is the actual steam reforming step, forming both hydrogen and carbon monoxide. 


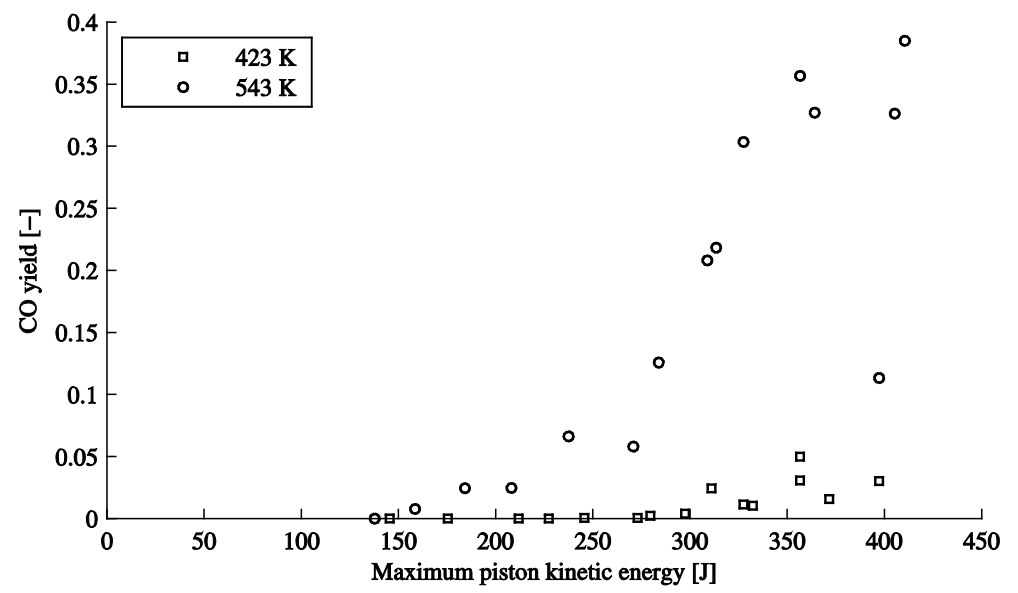

Figure 5.19, Carbon monoxide yield of experiments with mixture 4 with different temperatures

\subsubsection{Minor species}

As with the partial oxidation experiments, the minor species formed in the steam reforming experiments are shown as a function of methane conversion. This is done in Figure 5.20. The carbon dioxide yields of the steam reforming experiments with mixture 4 are almost nil. The highest carbon dioxide yield is found at the highest methane conversion levels, peaking to about $0.6 \%$. The acetylene yield is highest in the middle of the methane conversion scale, with a highest yield of around $10 \%$, and drops back to zero at nearly full methane conversion. The highest ethylene yield of around $12 \%$ is found just after the peak acetylene yield, around $60 \%$ methane conversion. The ethylene yield also drops to zero at near complete methane conversion. Ethane yield is highest at the end of the cycle, just before complete conversion of methane, around $80 \%$ conversion, with a highest measured yield of $1.4 \%$. The ethane yield drops, just like ethylene and acetylene to zero at near complete methane conversion. 

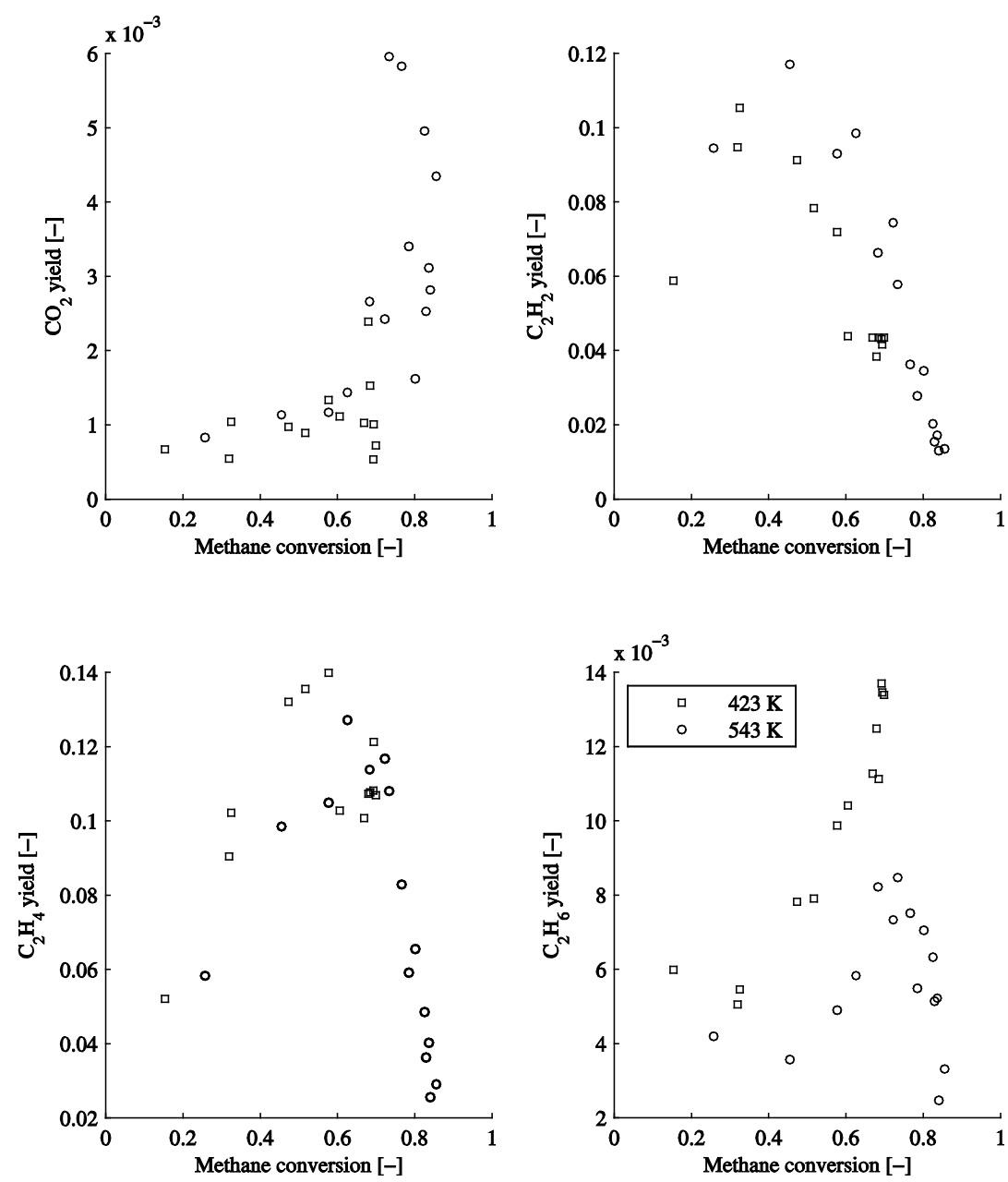

Figure 5.20, Minor species yield by experiment with mixture 4, different initial temperatures: $\mathrm{CO}_{2}$ (top left), $\mathrm{C}_{2} \mathrm{H}_{2}$ (top right), $\mathrm{C}_{2} \mathrm{H}_{4}$ (bottom left) and $\mathrm{C}_{2} \mathrm{H}_{6}$ (bottom right)

\section{Carbon, hydrogen and oxygen balance}

In the same way as this was done in the partial oxidation results the carbon, hydrogen and oxygen balance can be used to calculate soot formation, and the water mole fraction in the product gas. The results of this calculation are shown in Figure 5.21. Similar to the partial oxidation results, the soot yield is higher for higher methane conversion levels. However, in this case, it is not clear whether the soot yield drops back to zero when approaching full conversion of methane. Also the level of soot formation is much higher than in the partial oxidation experiments. 

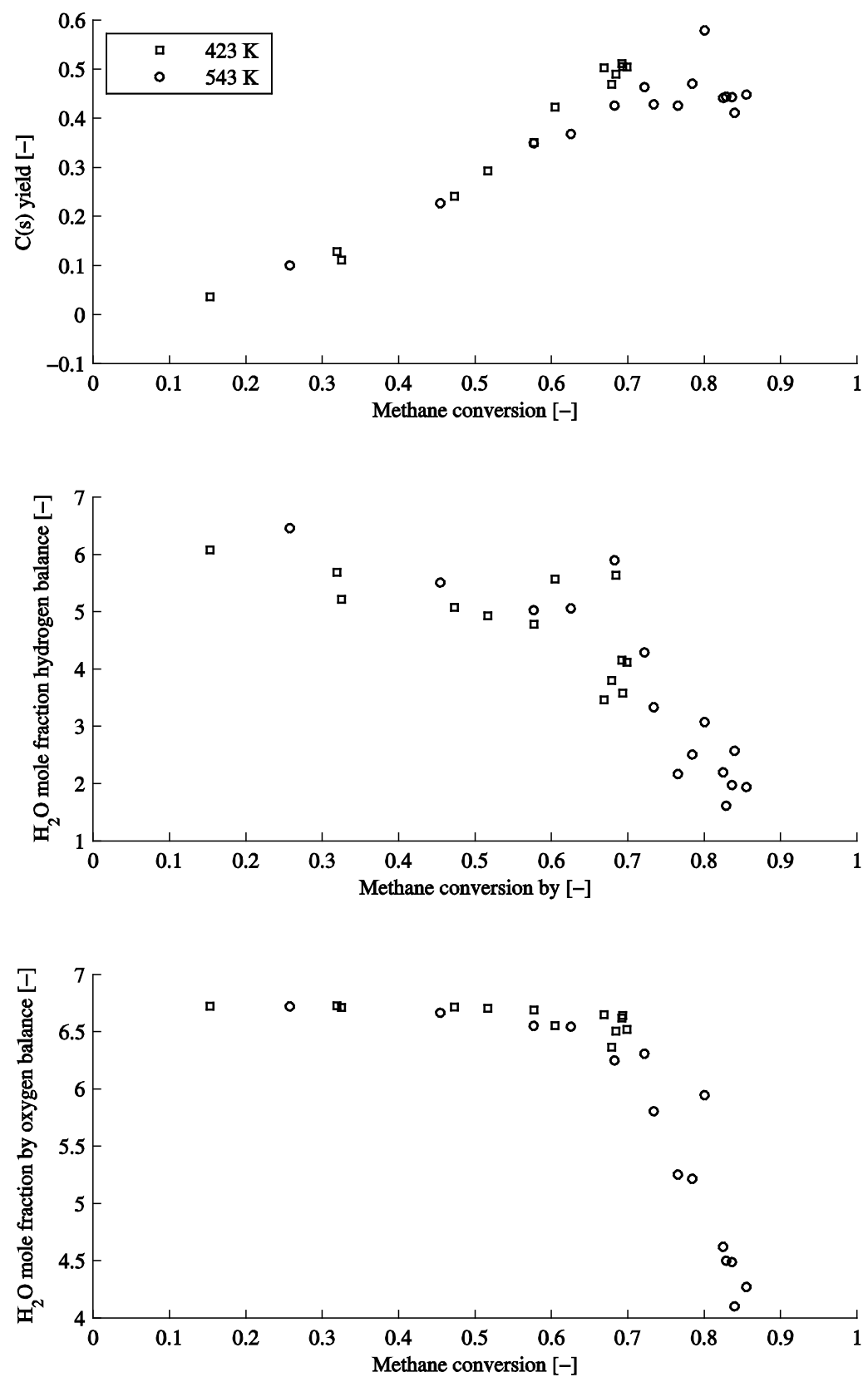

Figure 5.21, Water and soot yield, calculated from the carbon, hydrogen and oxygen balance 
The two different ways of calculating the water mole fraction in the product gas give similar results, but not entirely identical. The reason for the difference in results lies in the method of calculation. The calculation of the water mole fraction in the product gas based on the oxygen balance needs only the mole fraction of carbon dioxide and carbon monoxide as input variables. The initial water mole fraction is assumed to be the same for each experiment, giving very noise free results (since the carbon monoxide and carbon dioxide mole fractions are nearly zero for most experiments). The calculation of the water mole fraction based on the hydrogen balance on the other hand contains much more measurement noise, since for this calculation the mole fractions of all hydrogen containing species are used, and their concentrations are much higher. The result is that for some measurements, mole fractions of water in the product are found to be even higher than the assumed mole fraction of water in the reactants. Since this is highly unlikely, it may be assumed that these found values are erroneous and the result of measurement uncertainties.

\subsection{Chemistry simulation}

\subsubsection{Introduction}

The use of transient numerical simulations of the chemical reaction process within the single shot reactor allows one to study not only the product composition as a function of various parameters, but also the phenomena that occur during the compression expansion cycle. Conditions like the composition of the reactants or the initial temperature can be varied easily in simulations to study the effect of them, much more easily than conducting a new series of experiments. A numerical simulation also allows one to do simulations at elevated initial pressure, something that is not easily possible with the experimental setup that was used.

It may be clear that simulating the reaction process within the single shot reactor can have major benefits for the development of the PCR, but before one relies too much on numerical results, the simulation must be validated with experimental result. In the following sections, the developed numerical model will be explained and results of the model compared to the experimental data obtained.

\subsubsection{Numerical model}

For the simulation of the PCR single shot process, the open source software Cantera is used. Within this software the single shot PCR is modeled as a continuous stirred tank reactor (CSTR). The advantage of modeling the PCR in such a way is that no transport equations have to be solved, while still being able to model energy and mass exchange 
with the surrounding and volume variations. The version used for the simulations in this thesis is Cantera 1.7.0 for a 32-bit Windows operation system.

The core of the reactor model is the zero dimensional CSTR, where all information, like concentrations, temperature and pressure is lumped to a single point that represents the entire reactor. The reactor contains a predefined, ideal gas mixture. During the simulation, different reaction mechanisms can be used to simulate the interaction between the different chemical species. The number of species and reactions that are modeled depends on the reaction mechanism that is used.

The model solves a system of equations that determine the state of the reactor. This process is described in great detail in the report of Verbeek [64].

\subsubsection{Reaction mechanisms}

Since the conditions in the PCR are much extremer than the conditions that common reaction mechanisms are valid for, the performance of several different reaction mechanisms is tested. In Table 5.3 a summary of the used reaction mechanisms is shown, with the number of species and reactions that are modeled by the mechanism.

\begin{tabular}{lcc}
\hline \hline Mechanism & Species & Reactions \\
\hline GRI3.0 [65] & 53 & 325 \\
C3 [66] & 118 & 663 \\
C5 [67] & 132 & 1563 \\
Leeds [68] & 37 & 175 \\
Konnov [69] & 127 & 1207 \\
\hline \hline
\end{tabular}

Table 5.3, Reaction mechanisms used

The reaction mechanisms were all developed for the simulation of methane combustion. This means that the temperatures to which these models have been validated are in the order of $2200 \mathrm{~K}$ (the order of magnitude of the adiabatic flame temperature), but the pressures to which these models have been validated are only in the order of 10 to 25 bar. This means that it is possible that the mechanisms used will not yield accurate results when simulating the PCR process.

\subsubsection{Piston motion}

The compression expansion of the reaction chamber is modeled by changing the volume of the reaction chamber in the model. This volume change is achieved by modeling a wall with a certain surface area and specifying its velocity. The surface area of the wall in the model is taken to equal the top surface area of the piston. 
To calculate the velocity of the wall in the model, the equations of motion are solved for each time step:

$$
\begin{gathered}
a=\frac{A}{m}\left(P_{b}-P_{a}\right), \\
v=v+a \Delta t, \\
x=x+v \Delta t .
\end{gathered}
$$

To solve these equations of motion, the pressure above and below the piston are needed. The pressure above the piston is taken from the reactor model in Cantera. For the pressure below the piston a characteristic, measured pressure profile from the experiments is used. This profile is stored as a function of the piston position, and then scaled in order to simulate different launch pressures. In this way, the whole range of launch pressures can be simulated accurately, without needing to use different measured profiles for each simulation.

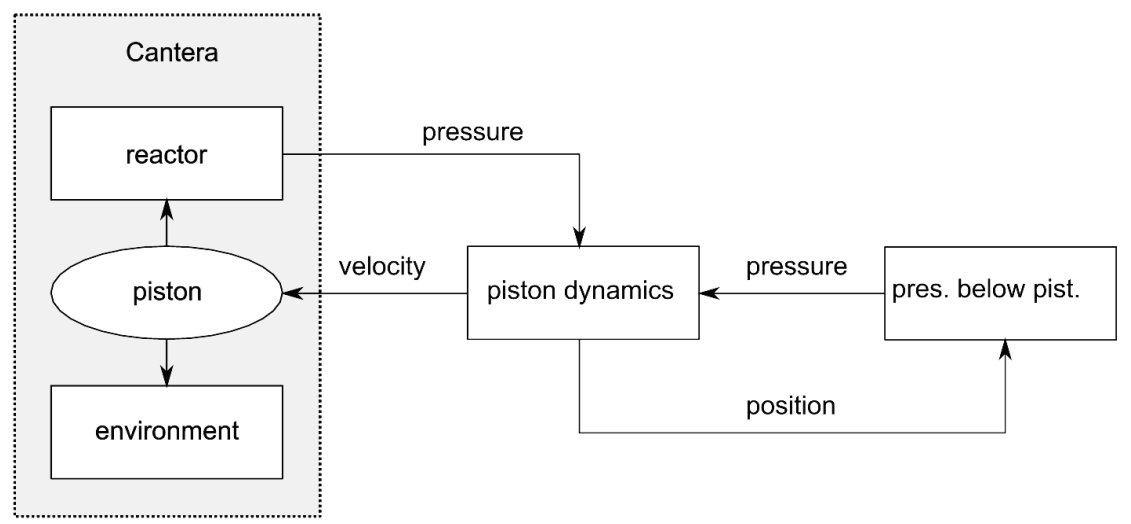

Figure 5.22, A schematic representation of the data flow in the simulations

Figure 5.22 shows the flow of data as it occurs in the model. The piston velocity is the input parameter for the Cantera reactor model and the pressure in the reactor is the output for each time step.

\subsection{Simulation results}

\subsubsection{Simulation of pressure and temperature}

A first and simple comparison between experiment and simulation is the comparison of the pressure that is predicted by the simulation to the pressure measured in the 
experiment. In Figure 5.23 this is done for the case where mixture 1 is preheated to $423 \mathrm{~K}$ and compressed by the piston with a maximum kinetic energy of $177 \mathrm{~J}$. The simulation shown uses the GRI 3.0 mechanism. This is the same experiment that was shown previously (with others) in Figure 5.3.

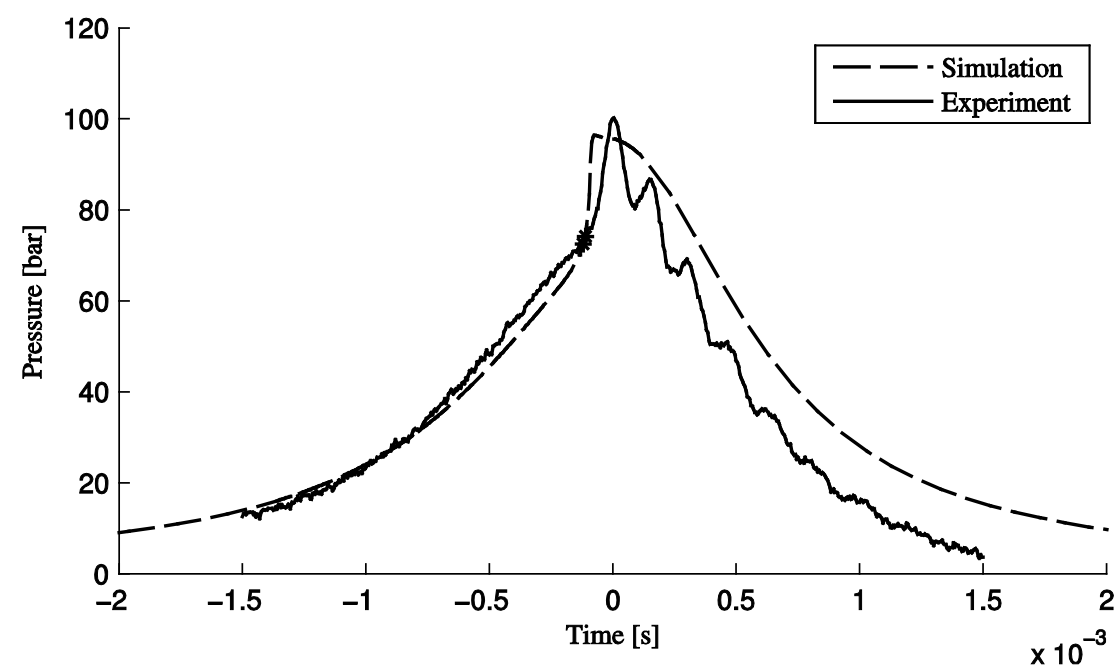

Figure 5.23, Simulated pressure compared to measured pressure, maximum piston kinetic energy $177 \mathrm{~J}$, initial temperature $423 \mathrm{~K}$, mixture 1

The comparison of experiment and simulation shows a very close resemblance, both in peak pressure attained, as well as in the moment of ignition (estimated in the figure by an asterisk). The main difference between the experiment and simulation is the pressure which the reactor gas returns to. This pressure is much higher in the simulations than in the experiments. This is most likely explained by the leakage along the piston in the experiments. This leakage is highest under peak compression and reduces the total mass of gas in the reactor. When the reactor expands, the pressure of the experiment is lower due to the lower total mass of gas in the reactor. Since this loss is not simulated, the mass of gas in the simulated reactor is the same before as after compression.

After comparing the pressure predicted by the simulation to the experiment, one can take a look at the predicted temperature. This is done in Figure 5.24. The temperature graph shows some resemblance to the pressure graph, with the most important difference that while the pressure graph is almost horizontal just after the point of highest pressure, the temperature graph shows a small dip right after the point of highest temperature. This indicates that the chemistry of the reactions occurring, are 
predicted to be predominantly endothermic just after the first jump of temperature and pressure at the moment of ignition.

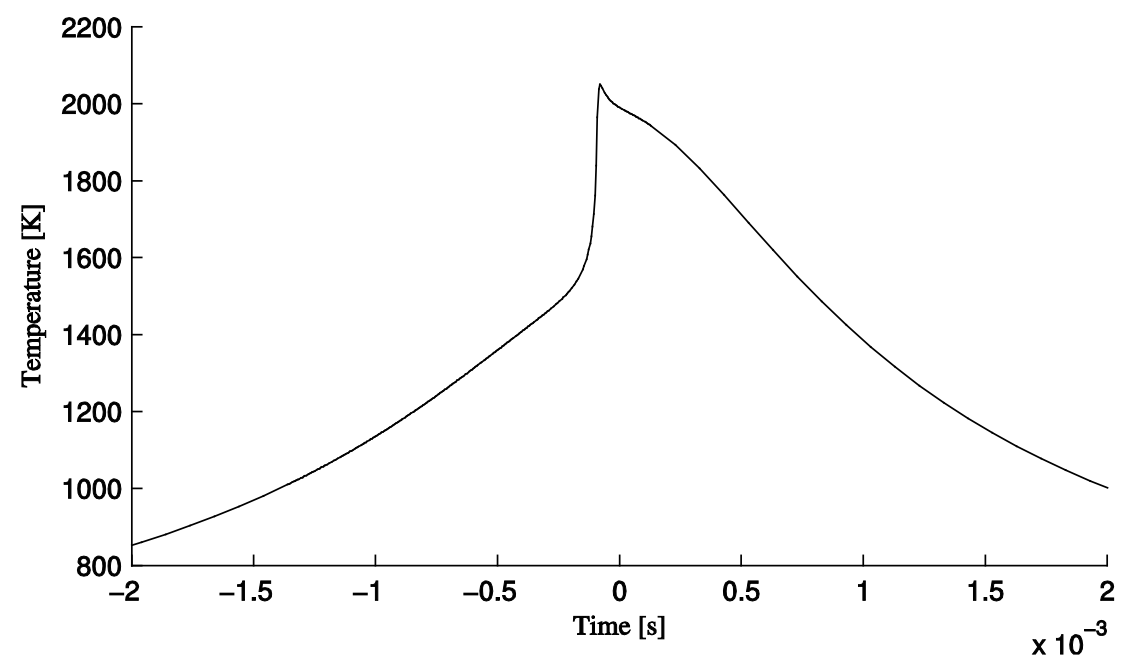

Figure 5.24, Simulated temperature, maximum piston kinetic energy $177 \mathrm{~J}$, initial temperature $423 \mathrm{~K}$, mixture 1

\subsubsection{Partial oxidation yields compared to measurements}

\section{Hydrogen yield}

In Figure 5.25 the yields of hydrogen of mixture 1 at different initial temperatures are compared to simulations with the same initial reaction mixture. In Figure 5.26 the yields of hydrogen with mixtures 2 and 3, at an initial temperature of $543 \mathrm{~K}$ are compared to simulations. When comparing the experimental results to the simulations done with the various models, a good correspondence between results is visible. However, especially for the higher temperature experiments, all models predict the threshold energy input to be slightly lower than is observed experimentally. Also, the observed yield in the experiments, for energy inputs well beyond the threshold energy, is lower than the predicted yield. The different models used give very similar results in terms of yield and threshold energy, no clear "best" model is visible (at least not in terms of predicting the hydrogen yield). 

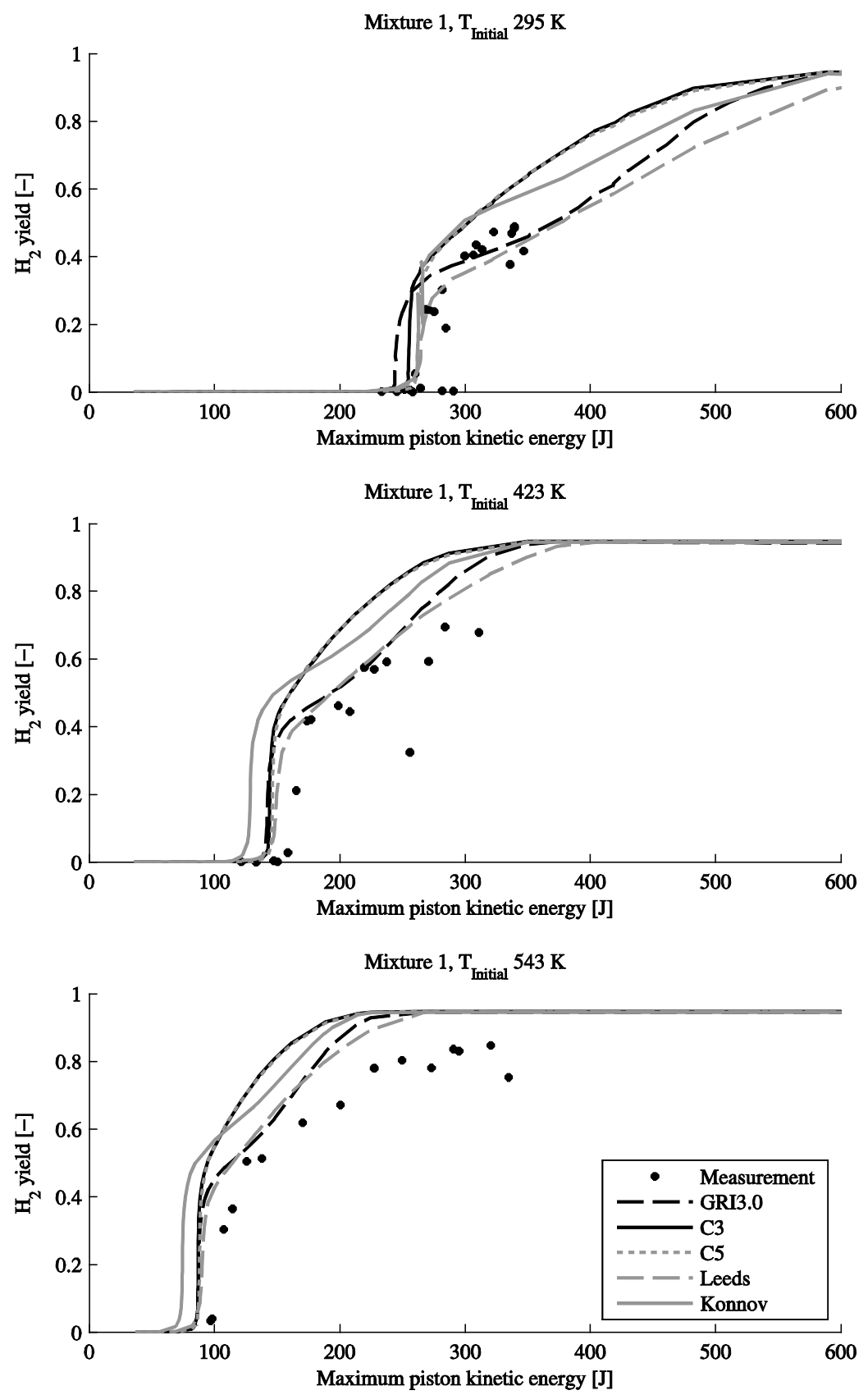

Figure 5.25, Hydrogen yield of experiments with mixture 1 and initial temperature $295 \mathrm{~K}$ (top), $423 \mathrm{~K}$ (middle) and $543 \mathrm{~K}$ (bottom), compared to simulations 

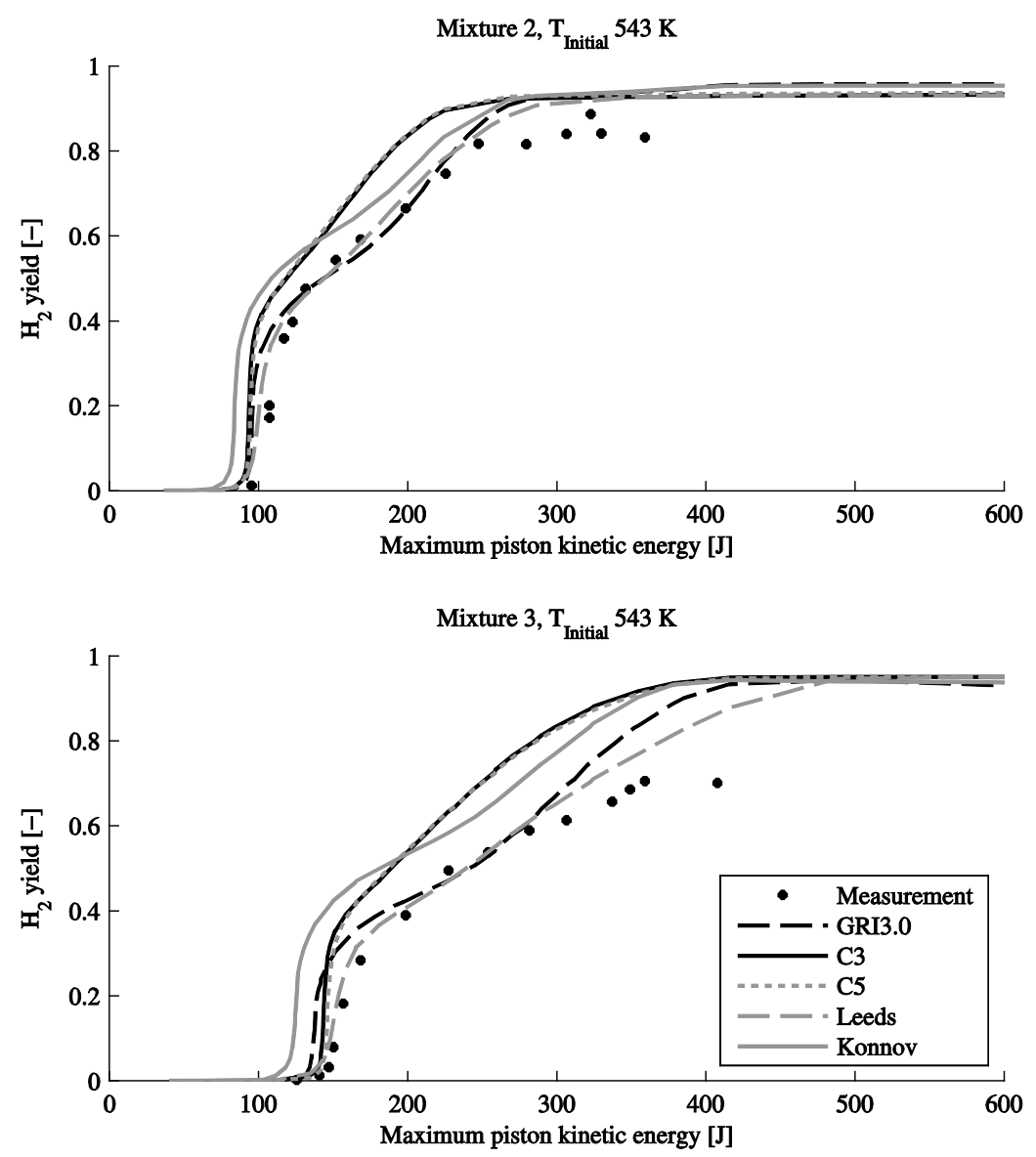

Figure 5.26, Hydrogen yield of experiments with mixture 2 (top) and 3 (bottom) and initial temperature $543 \mathrm{~K}$, compared to simulations

\section{Carbon monoxide yield}

In Figure 5.27 the yields of carbon monoxide of mixture 1 at different initial temperatures are compared to simulations with the same initial reaction mixture. In Figure 5.28 the yields of carbon monoxide with mixtures 2 and 3 , at an initial temperature of $543 \mathrm{~K}$ are compared to simulations. When comparing the carbon monoxide experimental results to the simulations again a good agreement is observed. While the threshold is again predicted slightly lower, trends are predicted well. The jump to about $40 \%$ yield at the threshold is predicted well, as well as the gradual increase after this jump. 

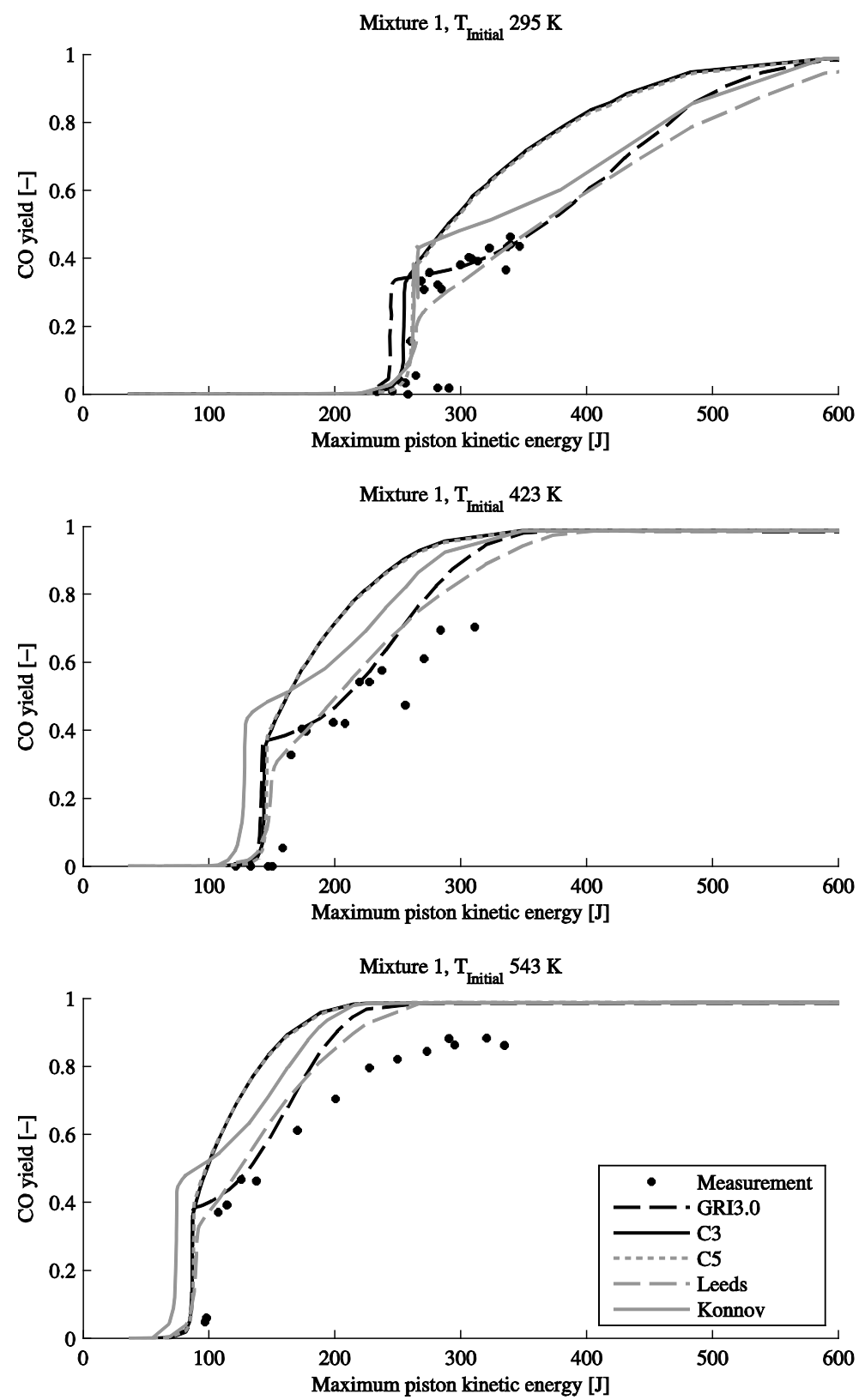

Figure 5.27, Carbon monoxide yield of experiments with mixture 1 and initial temperature $295 \mathrm{~K}$ (top), $423 \mathrm{~K}$ (middle) and $543 \mathrm{~K}$ (bottom), compared to simulations 

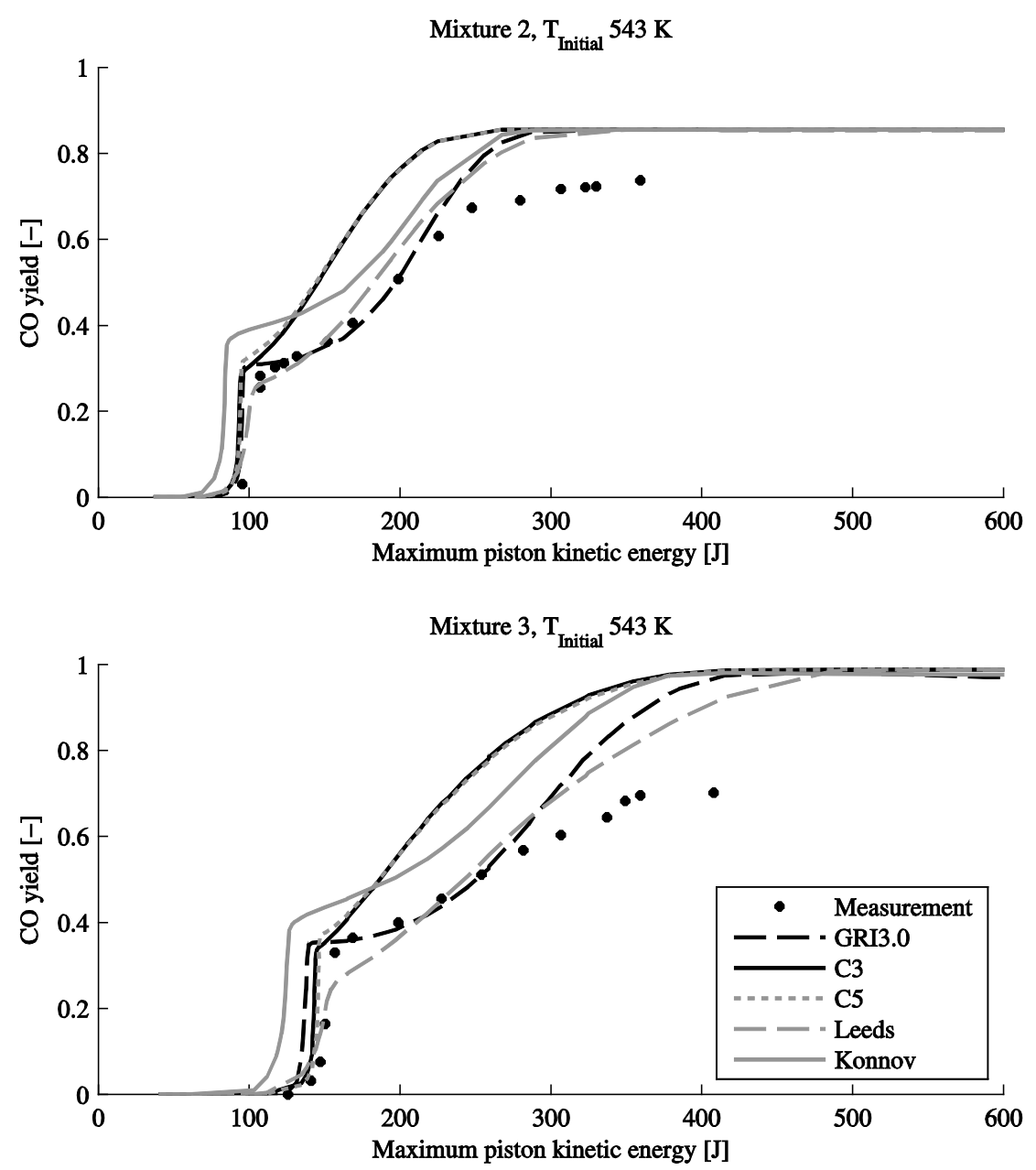

Figure 5.28, Carbon monoxide yield of experiments with mixture 2 (top) and 3 (bottom) and initial temperature $543 \mathrm{~K}$, compared to simulations

Comparing the different models to each other shows that the GRI 3.0 and Konnov model predict an almost horizontal carbon monoxide yield profile just after the threshold, while the other models predict a more smooth transition from the jump made at the threshold to the final maximum yield. Especially the experimental results from the $295 \mathrm{~K}$ initial temperature show that the profiles predicted by the GRI 3.0 and Konnov models are more accurate. Compared to the simulations, the same trends are observed as with the previous results. When comparing different models to each other, it visible that for the higher oxygen content mixture (mixture 2) the sharper angle between the threshold 
jump and yields just after the threshold has also become visible in the C3, C5 and Leeds model. Although this sharp angle is still more prevalently present in the GRI 3.0 and Konnov model.

\section{Minor species}

Figure 5.29 shows the yield of some minor species of the experiment series using mixture 3 with initial temperature $543 \mathrm{~K}$. The data is represented against the methane conversion; the same was as was done in Figure 5.12. The reason that this specific series is chosen to use to validate the numerical models is that the method of plotting that is used (plotting yield against methane conversion) stretches the ignition moment over a large part of the scale. The jump in yields that is seen in all data, both experimental as well as numerical, is also a jump from low methane conversion (less than $25 \%$ ) to high methane conversion (more than 75\%). This means that to make sure that not all measurement data is concentrated on the left and right side of the graph, with no data in the middle, a measurement series is required that has several measurement points within the jump. The data obtained with mixture 3 most qualifies for this.

The predictions of the formation of minor species by the various reaction mechanisms show a much bigger difference between different reaction mechanisms. For example, in the prediction of acetylene formation, the Konnov mechanism predicts a maximum yield of $8 \%$, while the other mechanisms are closer to $20 \%$ or even $30 \%$. In this case the Konnov mechanism seems to be the most accurate predictor. Whether or not the shape of the Konnov mechanism, a truncated peak, or the shape of the other mechanisms, a uniform peak, is more accurate is not entirely clear from the experimental results, since there is no measurement point at a methane conversion of $60 \%$.

In the ethylene yield prediction, the same difference between the Konnov mechanism and the other four is found, and again the results are closest to the Konnov prediction. The curve shape with two peaks, predicted by all mechanisms, is found also in the experimental results.

The ethane predicted shape, a large concentration in the beginning, with a smaller peak near the end, is confirmed by experiments. The Leeds and Konnov mechanism are closest in terms of absolute yield.

An attribute that is shared by all the minor species figures is that the experimental data for high conversions (the rightmost points in the graphs) is shifted along the methane conversion axis around $10-20 \%$. This difference between experiment and simulation is most likely caused by the fact that simulations are not affected by the presence of walls: 
all reactants are ideally mixed and the same temperature. In the experiments on the other hand, some of the reactants, those close to the wall, are cooled by the wall and do not react. The result is that the final composition of the product is a mixture of completely reacted gas from the core of the reactor and unreacted gas from the boundary layer. This effect manifests itself in a shift along the methane conversion axis: the unreacted methane dilutes the products.
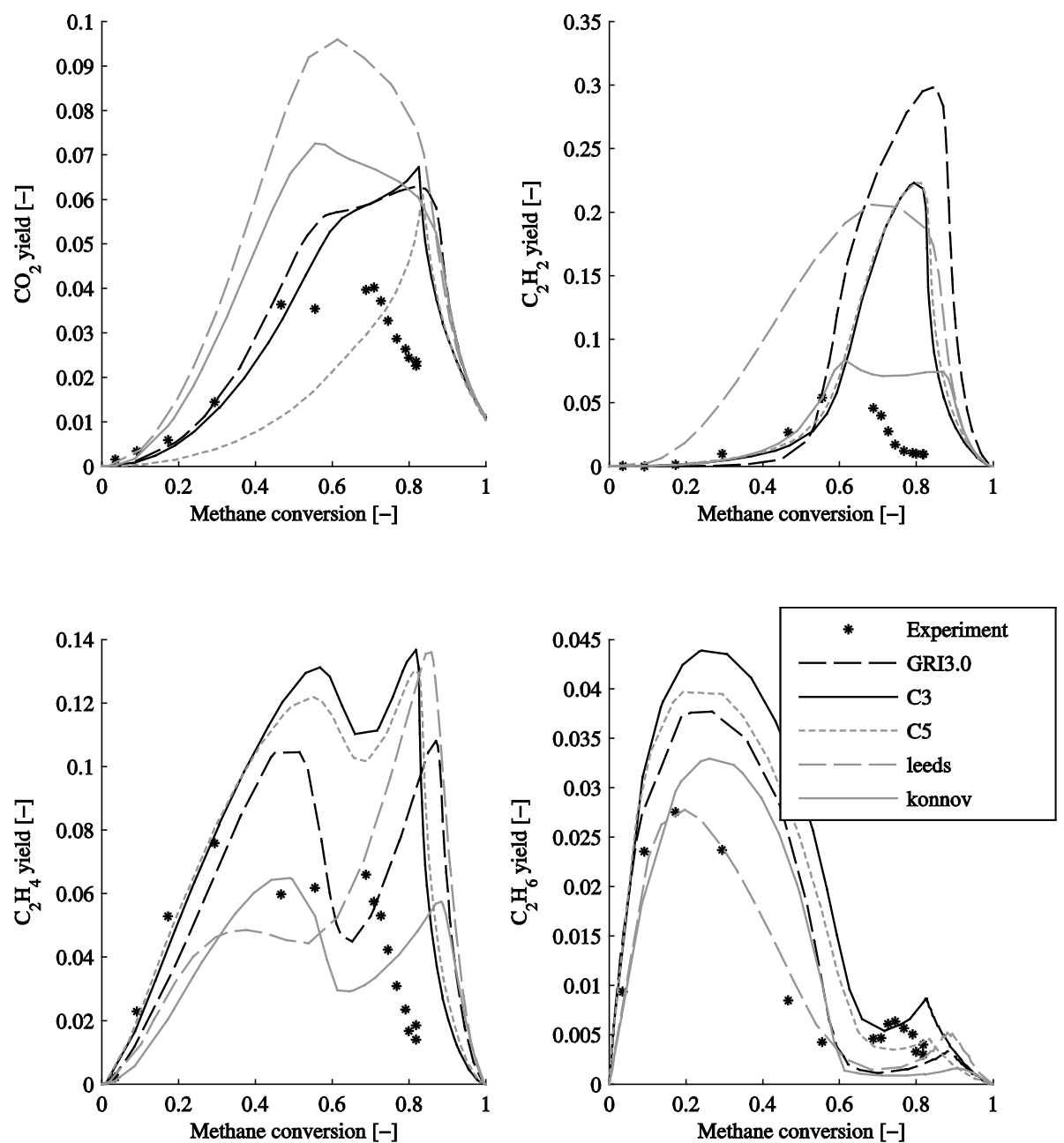

Figure 5.29, Minor species yield by experiment with mixture 3, initial temperature $543 \mathrm{~K}: \mathrm{CO}_{2}$ (top left), $\mathrm{C}_{2} \mathrm{H}_{2}$ (top right), $\mathrm{C}_{2} \mathrm{H}_{4}$ (bottom left) and $\mathrm{C}_{2} \mathrm{H}_{6}$ (bottom), compared to simulations 
Concerning the last minor specie, carbon dioxide, the predictions of all models are pretty close to each other, while the experimental results are much lower. The shape of the curve that the experimental results show though, is not well predicted by the C5 mechanism. Interestingly, the prediction of carbon dioxide formation is the only point where there is a large visible difference between the C3 and C5 mechanism (in all hydrogen and carbon monoxide graphs, the C3 and C5 curves are nearly identical).

From these results it may be concluded that the Konnov mechanism is closest in the prediction of the minor species, but results are not as close as the prediction of the major species. The shape of the formation curves are well predicted by all mechanisms (with the exception of the carbon dioxide prediction by the C5 model). The Konnov mechanism is closest in terms of absolute concentration for most of the minor species.

\subsubsection{Hydrogen - carbon monoxide ratio}

The importance of the hydrogen - carbon monoxide ratio has been stressed before. Whether or not the simulation models are able to predict this ratio accurately can be seen in Figure 5.30. In this figure the hydrogen - carbon monoxide ratio of the $543 \mathrm{~K}$ series of each of the mixtures is plotted and compared to the simulated ratio.

While the simulations do not show great differences between different models, an aspect that is not surprising, since there was not much difference between models in predicting the hydrogen and carbon monoxide yield, the correspondence of the predicted ratio with experiments is good. Again there is a small shift of the high conversion data along the conversion axis. Besides this difference, the predicted hydrogen - carbon monoxide ratio by the different models is remarkably close to the measured ratio, both in value as well as in the relation between the ratio and the conversion of methane.

In part of the curves in Figure 5.30 that is most interesting for the operation of the PCR, namely the top $40 \%$ of the methane conversion axis, the shape of the hydrogen - carbon monoxide ratio curves of mixtures 1 and 2 is comparable, but the mixture 2 curve is stretched along the hydrogen - carbon monoxide ratio axis. This adds to the tuneability of the hydrogen - carbon monoxide ratio. 

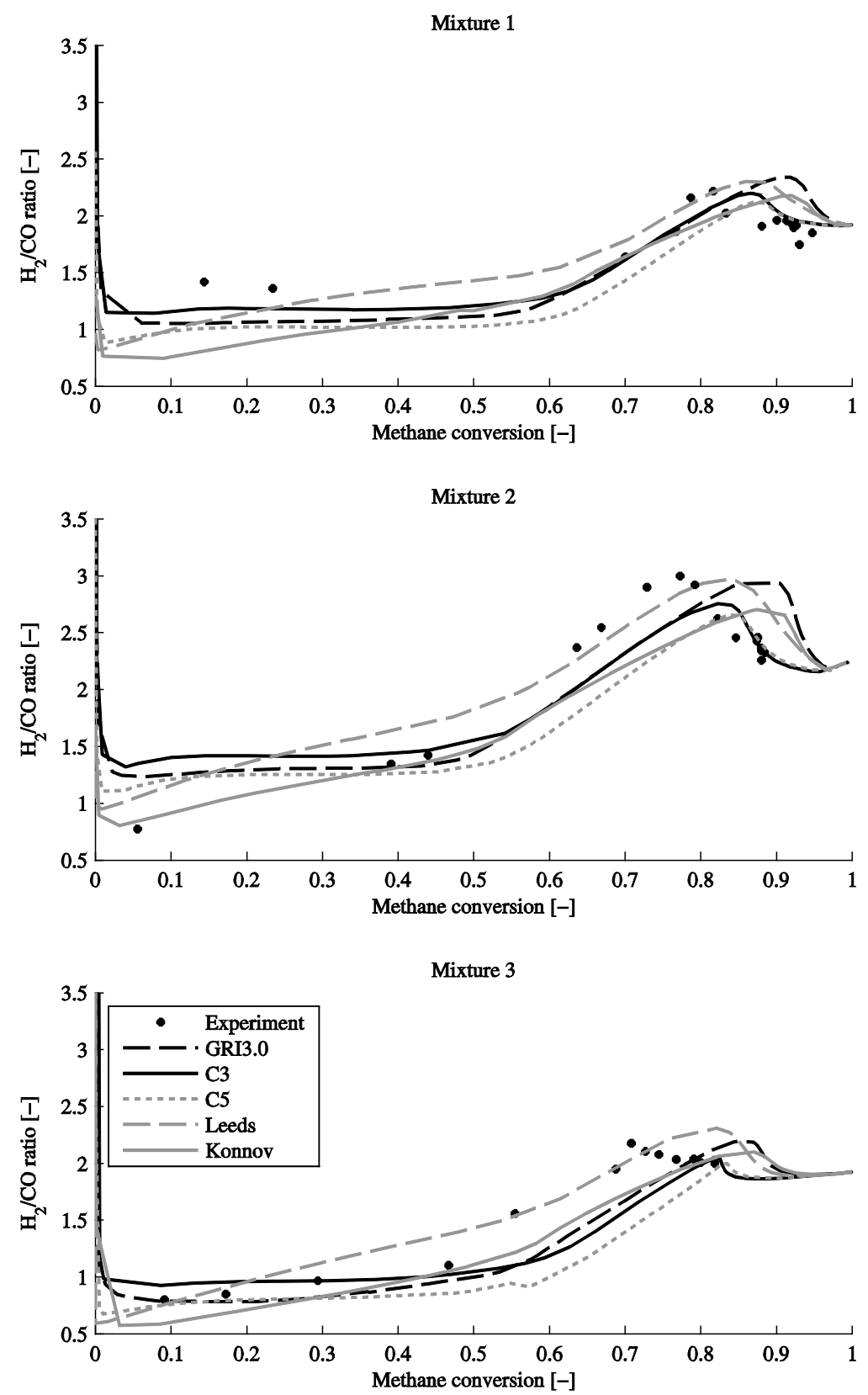

Figure 5.30, Hydrogen - carbon monoxide ratio, for mixture 1 (top), mixture 2 (middle) and mixture 3 (bottom), initial temperature $543 \mathrm{~K}$, compared to simulations 


\subsubsection{The partial oxidation process}

One of the key reasons for developing a numerical model is to determine exactly what happens with the chemical composition of the reaction mixture during one compression expansion cycle, since experimental results can only give initial and final compositions.

In Figure 5.31 the major species of during a simulated compression expansion cycle are shown. The initial composition of the simulation was 10 vol\% $\mathrm{CH}_{4}, 5$ vol\% $\mathrm{O}_{2}, 15$ vol\% $\mathrm{N}_{2}$ and $65 \mathrm{vol} \% \mathrm{Ar}$. The initial temperature of the simulation was $543 \mathrm{~K}$ and the maximum piston kinetic energy $350 \mathrm{~J}$. Results are shifted in time to make $\mathrm{t}=0$ the time at which the pressure is maximum (as is done in Figure 5.3).

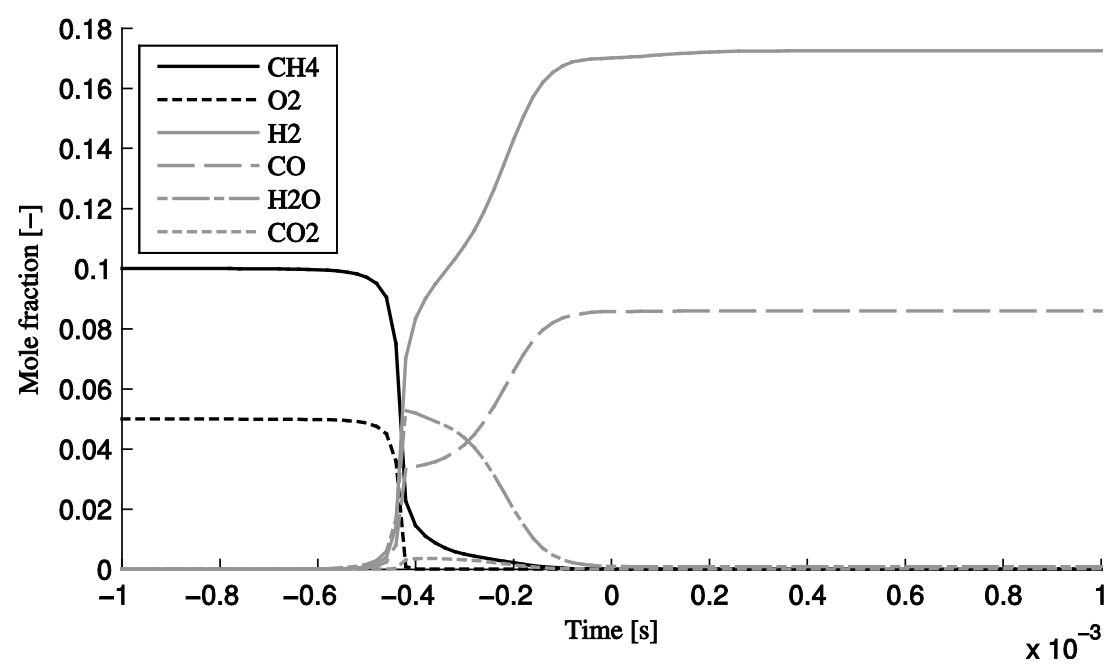

Figure 5.31, Major species and carbon dioxide during compression expansion cycle with maximum piston kinetic energy $350 \mathrm{~J}$, simulated by the GRI3.0 mechanism. Initial temperature $543 \mathrm{~K}$

The evolution of the major species as found by the simulation show that the reaction progress can be divided in two steps. The first step is a combustion like step. In this step, which is very short, all of the oxygen present is consumed with the bulk of the methane, and converted to water, hydrogen, carbon monoxide and some carbon dioxide. In the second step, which is much slower, the water produced in the first step reforms the left over methane to more hydrogen and carbon monoxide.

The understanding of these two consecutive steps also explains the experimental results. When little energy is supplied, a piston kinetic energy just over the threshold, only the first combustion step occurs. As more energy is supplied, the total cycle length becomes 
shorter, while at the same time, peak temperatures are higher. The higher temperatures speed up the chemistry, so in fact, while the cycle length is shorter, faster reaction rates means that the second step will be able to proceed further. This mechanism is confirmed by the experimental results, where there is a base production of hydrogen and carbon monoxide when the threshold kinetic energy is exceeded, followed by a more gradual increase in yield as the piston kinetic energy is increased further.

An important observation is the striking resemblance the curves show to the yield curves for different energy inputs. This leads to the conclusion that the quenching rate of the $P C R$ is indeed high enough to freeze the reaction products in a non equilibrium state. If little energy is supplied, the reactions still follow the path that is shown in Figure 5.31, but the process is stopped and reaction products frozen at, for example, just after the combustion like step.

Figure 5.32 shows the minor species during one compression expansion cycle, as predicted by the GRI3.0 model. The species shown all go back to zero as the cycle proceeds, but have peak values in the same order of magnitude as the predicted yields from cycles with a lower maximum piston kinetic energy. This again supports indicates that the rapid quenching rate of the PCR freezes reactions.

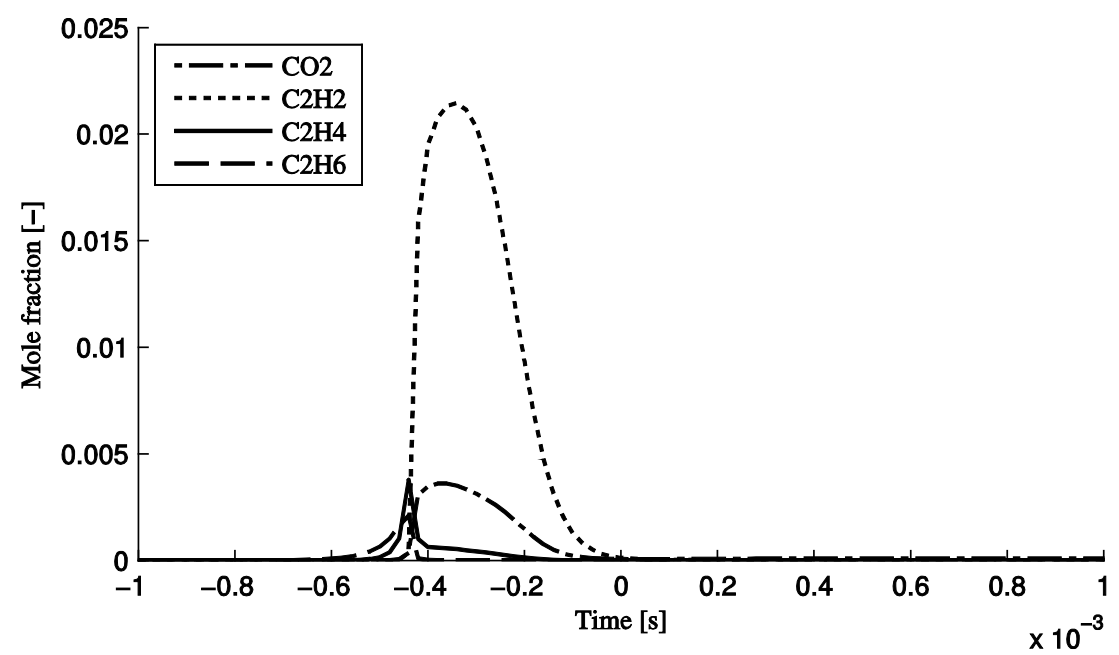

Figure 5.32, Minor species and carbon dioxide during compression expansion cycle with maximum piston kinetic energy $350 \mathrm{~J}$, simulated by the GRI3.0 mechanism. Initial temperature $543 \mathrm{~K}$ 


\section{Yield as a function of total energy input}

In order to be able the compare the experiments with different initial temperatures directly, one might plot the yield as a function of total energy input. This total energy input is comprised of the kinetic energy of the piston, which is in the process exchanged from the piston to the reaction gasses and the thermal energy that is added to the reactant gasses prior to the initiation of piston motion. Since all experiments start at ambient pressure, it is inherent that the density of the reactants is lower in the experiments with higher initial temperature. This means that for experiments with a higher initial temperature the kinetic energy of the piston is transferred to less mass of reactants. For this reason, when the total energy input is calculated for the different situations, it should be calculated as energy per mass of reactants (the specific energy input).

In Figure 5.33 the simulated hydrogen yield of mixture 1, with the three different initial temperatures used is plotted, as a function of specific total energy input. For the simulation with ambient initial temperature the specific total energy is only the maximum kinetic energy of the piston divided by the mass of reactants in the reactor. For the other two simulations it is the sum of piston kinetic energy and thermal energy input to preheat the reactants.

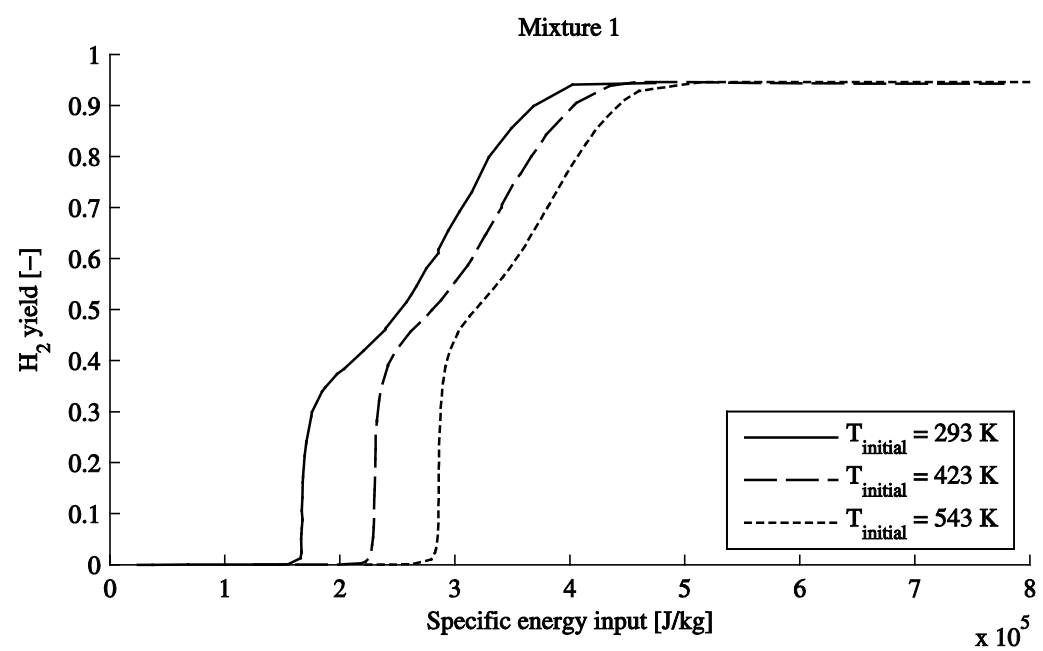

Figure 5.33, Hydrogen yield as a function of total specific energy input, simulated by the GRI3.0 mechanism

The specific energy required for ignition is lowest when the energy supplied consists only of kinetic energy of the piston, instead of a combination of kinetic energy of the piston 
and heat to the gas. This can be explained by the pressure dependence of the auto ignition temperature of the methane air mixture as is found, for example, by Caron, M., et al [70]. The auto ignition temperature of methane air mixtures decreases as pressure is increased. Since all the energy input in the simulation that starts from ambient temperature is used for compression, the peak compression pressure of this simulation is higher than that of the "preheated" simulations with identical total energy input. This means that, while the same total amount of energy is supplied, the mixtures that start from ambient temperature will ignite sooner.

\section{Off equilibrium product compositions}

One of the key strengths of the pulsed compression reactor is its rapid quenching rate which enables the freezing of intermediate reaction products in non equilibrium state. To see the effect of this strength in the case of the process under investigation in this section, the simulated concentrations in the reaction mixture are compared to two equilibrium concentrations in Figure 5.34. The reaction mixture simulated had an initial composition of 10 vol\% $\mathrm{CH}_{4}, 5$ vol\% $\mathrm{O}_{2}, 15$ vol\% $\mathrm{N}_{2}$ and 65 vol\% Ar, with an initial temperature of $423 \mathrm{~K}$.

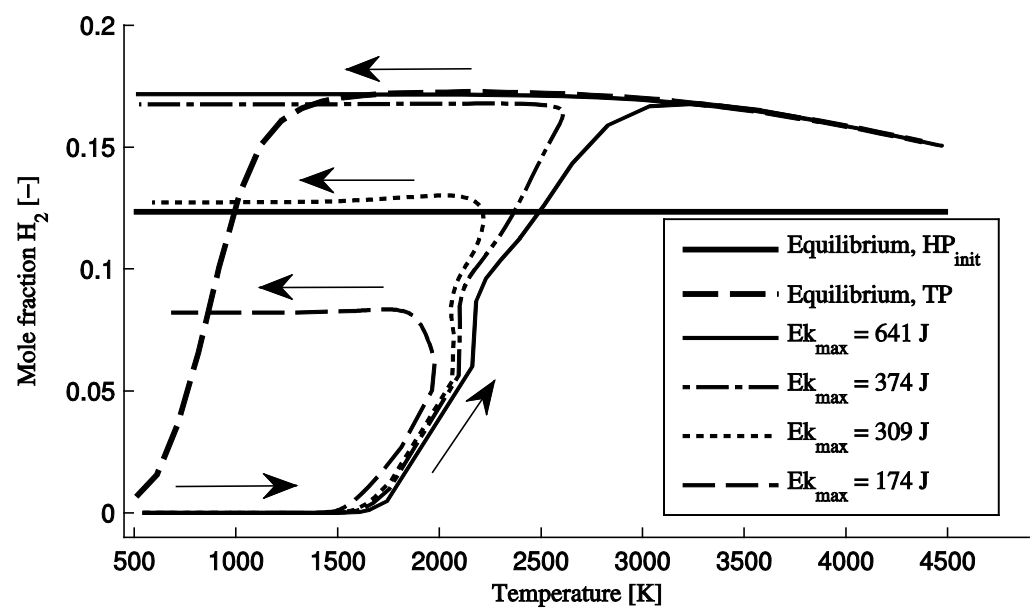

Figure 5.34, Hydrogen concentration during compression expansion cycle compared to equilibrium concentrations

Firstly, the equilibrium hydrogen concentration is shown, for the same specific enthalpy and pressure as the initial situation. This equilibrium concentration shows what the hydrogen yield would be if the mixture were allowed to react in a hypothetical adiabatic flame, at constant pressure, where the feed stream of the flame is the same as the initial mixture in the PCR. Since this is a fixed value, it is shown in Figure 5.34 as constant line. 
The second equilibrium that is used for comparison is the equilibrium hydrogen concentration at the different temperatures and pressures as they occur during one compression expansion cycle.

These two different equilibrium composition curves are compared to the hydrogen concentrations during four compression expansion cycles, with different maximum piston kinetic energies. They are shown as function of temperature. As seen before, increasing the maximum piston kinetic energy increases the final hydrogen concentration, to a limit. At a maximum piston kinetic energy of $174 \mathrm{~J}$ the hydrogen yield is less than the hypothetical adiabatic flame would yield. As the maximum piston kinetic energy is increased the hydrogen yield increases beyond yield of the hypothetical adiabatic flame. However, the hydrogen yield is limited by the equilibrium concentration at high temperature and pressure: the simulation with a maximum piston kinetic energy of $641 \mathrm{~J}$ shows the hydrogen concentration increasing until it matches the equilibrium concentration, after which it is frozen by quenching at this composition. This shows the great strength of the PCR: while the equilibrium hydrogen concentration is zero at low temperature and pressure, the yielded hydrogen concentration for a cycle with a maximum piston kinetic energy of only $374 \mathrm{~J}$ is more than $17 \mathrm{vol} \%$ : the equilibrium concentration at $2000 \mathrm{~K}$.

\section{Molar increase approximation}

In section 3.3.7 the method for approximating the molar increase of products with respect to reactants was introduced. This method has been consistently used to correct the measured values by the GC. However, a post check has to be made, in order to check whether the assumptions made on which the approximation was based was correct. In Figure 5.35 the exact molar increase of the simulations is plotted, together with the corresponding approximation used for the experiments. The standard deviation of the approximated molar increase to the simulated molar increase is $2.1 \times 10^{-3}$. The two sets of data correspond well enough to warrant the use of the approximation for the experiments. 


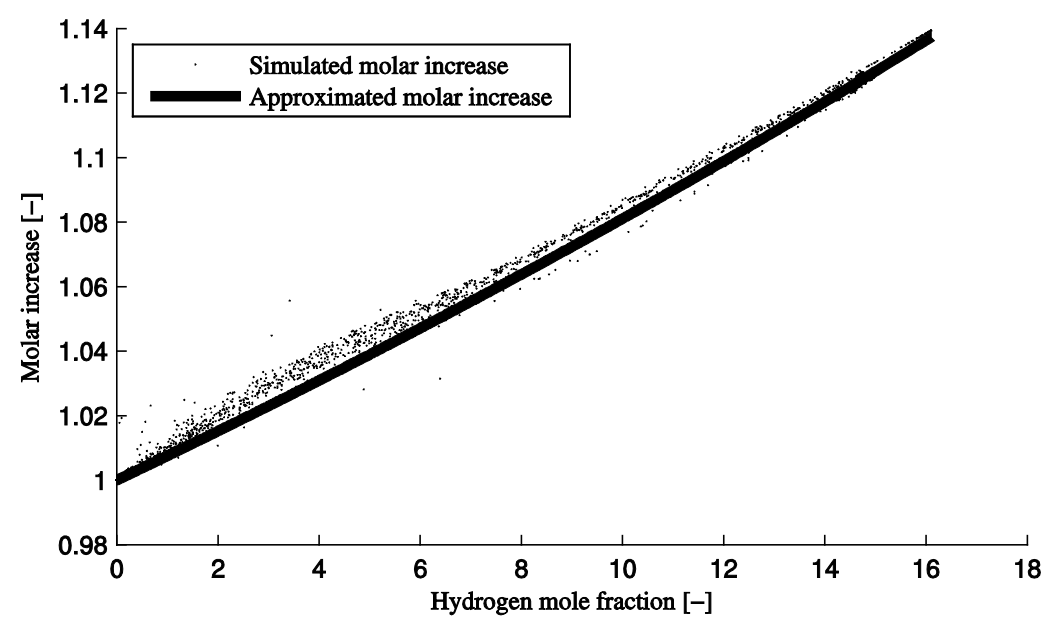

Figure 5.35, Simulated molar increase compared to molar increase by approximation

\subsubsection{Steam reforming yields}

\section{Hydrogen yield}

In Figure 5.36 the simulated hydrogen yield by steam reforming of mixture 4 is shown compared to the experiments. Of course, the room temperature simulation is not actually possible, since the steam concentration in the simulation is higher than the saturated water concentration at that temperature. However, the simulation at room temperature is included nonetheless, to enable the results to be compared well to the partial oxidation results.

The simulation results show that, as far as hydrogen yield is concerned, the different models give similar results in terms of minimum energy required to yield hydrogen. Since there is no oxygen present in the initial mixture, there is no sudden jump in hydrogen yield when combustion takes place. Instead the hydrogen concentration slowly starts rising at energy levels just beyond the partial oxidation threshold.

The division into two distinct formation parts, as could be seen in the experiments, is confirmed by the different models, although different models show different moments of transition of the first part to the second part.

The simulations generally agree well with the experimental results, both in energy required to start formation of hydrogen, as well as the achieved yield (though, similarly to the partial oxidation results, final yields are predicted to be higher). 

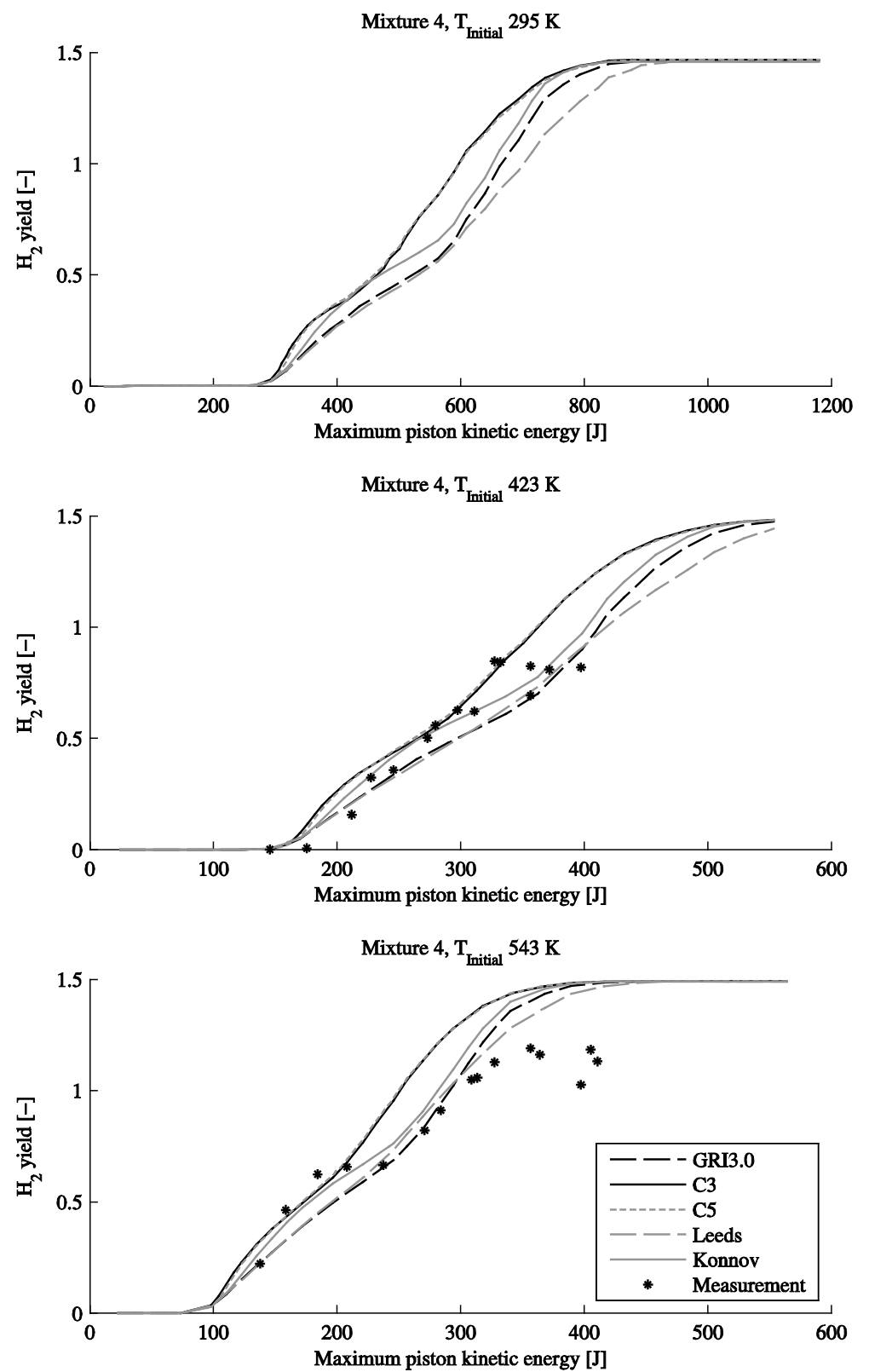

Figure 5.36, Hydrogen yield of simulations with mixture 4 and initial temperature $295 \mathrm{~K}$ (top), $423 \mathrm{~K}$ (middle) and $543 \mathrm{~K}$ (bottom) compared to experiments 

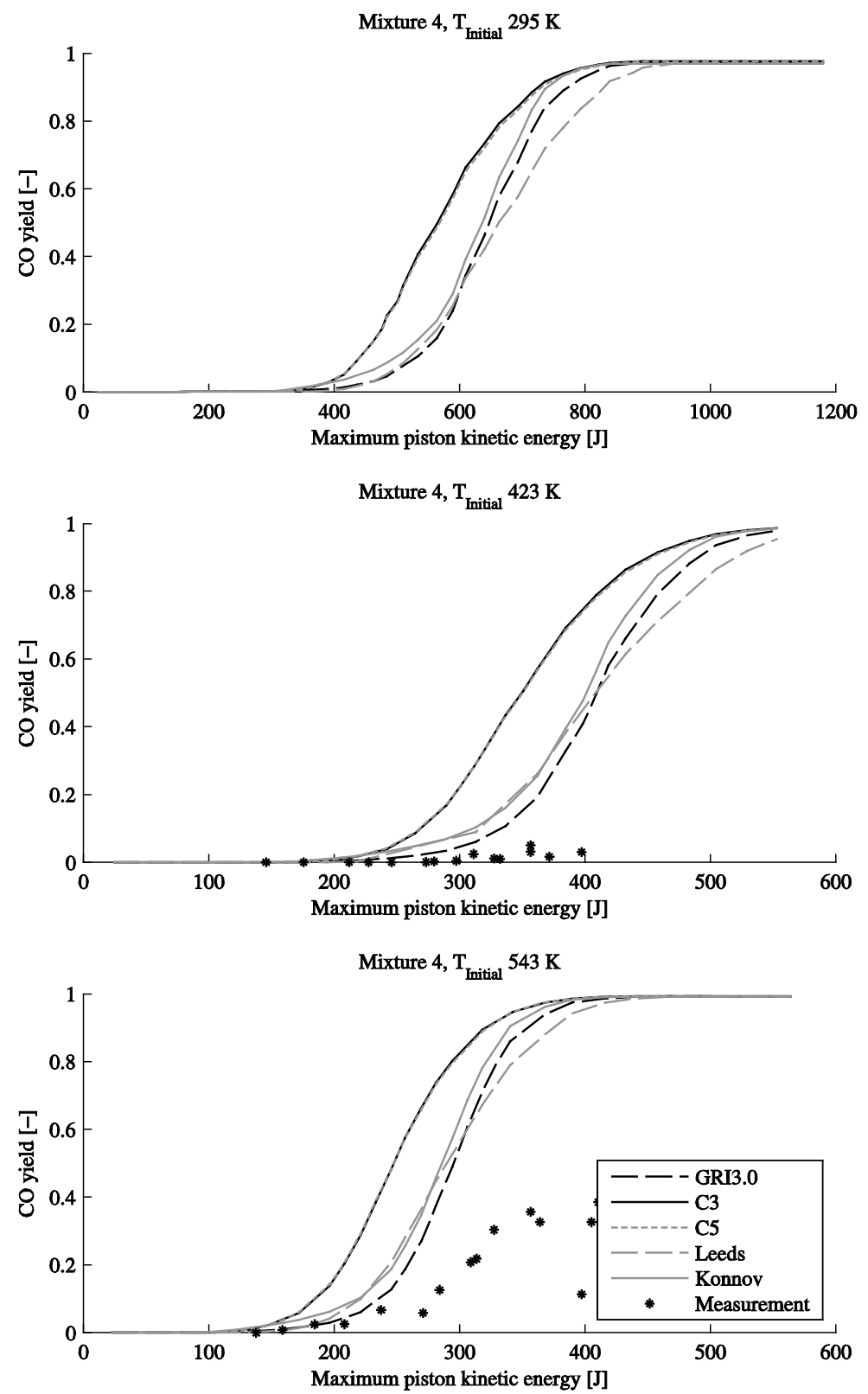

Figure 5.37, Carbon monoxide yield of simulations with mixture 4 and initial temperature $295 \mathrm{~K}$ (top), $423 \mathrm{~K}$ (middle) and $543 \mathrm{~K}$ (bottom) 


\section{Carbon monoxide yield}

The difference in predicted carbon monoxide yield between different models is much larger than the predicted hydrogen yield. The simulated carbon monoxide yields by steam reforming are shown in Figure 5.37. While results in terms of hydrogen yield are quite comparable between models, the difference between the C3 and C5 models and the other three are much larger when looking at the carbon monoxide yield. While the C3 and C5 models predict formation of carbon monoxide at piston energy levels just higher than the point where hydrogen is formed, the other three models predict that much more energy is needed for the formation of carbon monoxide.

This difference between the models was also visible in the partial oxidation results: the steam reforming step is predicted to occur immediately after the combustion like step by the C3 and C5 model, while the Konnov and GRI3.0 models predict no increase in carbon monoxide yield when the energy input is increased a little beyond the threshold energy. From the steam reforming results it becomes evident where this difference comes from: the C3 and C5 models predict that much less energy is required for steam reforming to occur.

The absence of carbon monoxide at energy levels where hydrogen is yielded leads to the conclusion that the decomposition of methane through the Kassel mechanism, as outlined in equation 5-1, occurs before the formation of carbon monoxide through steam reforming.

Compared to the experiments, the models, even those that predict more energy needed for steam reforming, predict carbon monoxide to be yielded at lower energy levels than the experiments. However, this difference is small compared to the difference in predicted yield. While the models predict a final carbon monoxide yield of around $88 \%$, the experiments reach no further than $35 \%$.

This difference can be explained by the absence of a soot formation model in the different reaction mechanisms used. The two different parts that were visible in both the hydrogen as well as the carbon monoxide results can be identified as a methane cracking step, followed by the actual steam reforming step. The cracking step forms, next to the found hydrogen, also a significant portion of soot. The formation of soot reduces the amount of carbon atoms that are available in the mixture for the yield of carbon monoxide, thus reducing the amount of carbon monoxide formed. The absence of a soot model in the used mechanisms makes much higher carbon monoxide yields possible. 


\section{Minor species}

If the conclusion that the decomposition of methane through the Kassel mechanism happens at lower energy levels than the formation of carbon monoxide through steam reforming, based on the carbon monoxide results, is indeed correct, then it should be visible in the ethane, ethylene and acetylene yield results. In Figure 5.38 the ethane, ethylene and acetylene yield results are shown, for the initial temperature of $543 \mathrm{~K}$. While the first significant yield of carbon monoxide does not occur before $150-200 \mathrm{~J}$, there is already significant ethane yield between 50 and $100 \mathrm{~J}$. This supports the theory that the decomposition of methane to ethane, ethylene and finally acetylene happens before significant steam reforming.

In Figure 5.39 the minor species are depicted in the same manner as was done for the partial oxidation results and compared to the experimental results. It is again evident that ethane is formed right at the beginning of the first conversion of methane. Another interesting result is the lack of carbon dioxide predicted. All models predict that only at one specific conversion a significant quantity of carbon dioxide is formed. And even then, it is much less than the yields that are obtained by partial oxidation.

The experimental results show, just like in the partial oxidation results, best correspondence to the Konnov mechanism. Yields of ethylene and acetylene are most well predicted by the Konnov mechanism, while the yield of ethylene on the other hand is predicted to be lower than the experimental results. In the case of ethylene the results correspond best to the Leeds mechanism. Experimental carbon dioxide is much lower than the predicted highest yield by all mechanisms. 

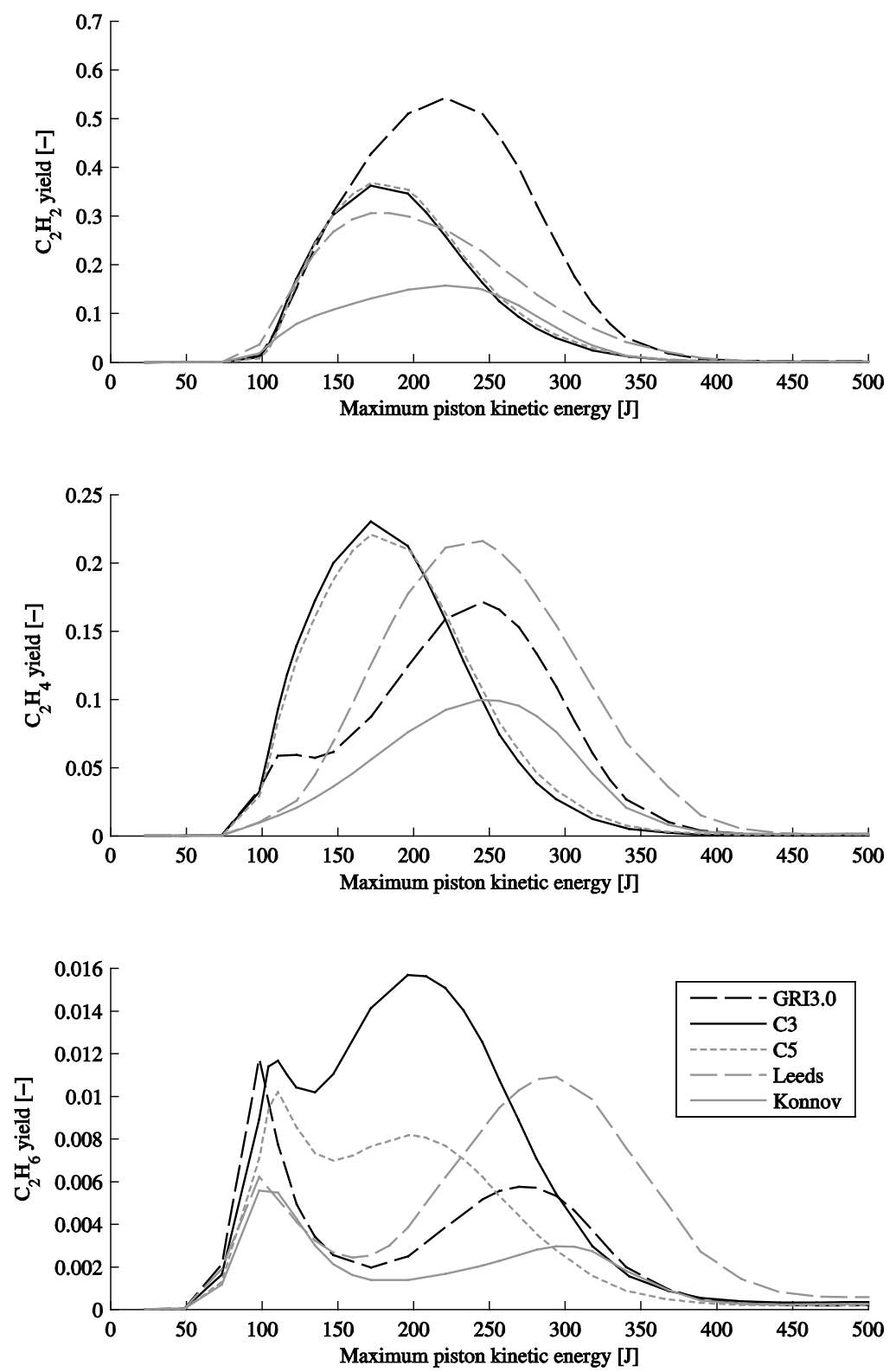

Figure 5.38, Acetylene (top), ethylene (middle) and ethane (bottom) yield of simulations with mixture 4 and initial temperature $543 \mathrm{~K}$ 

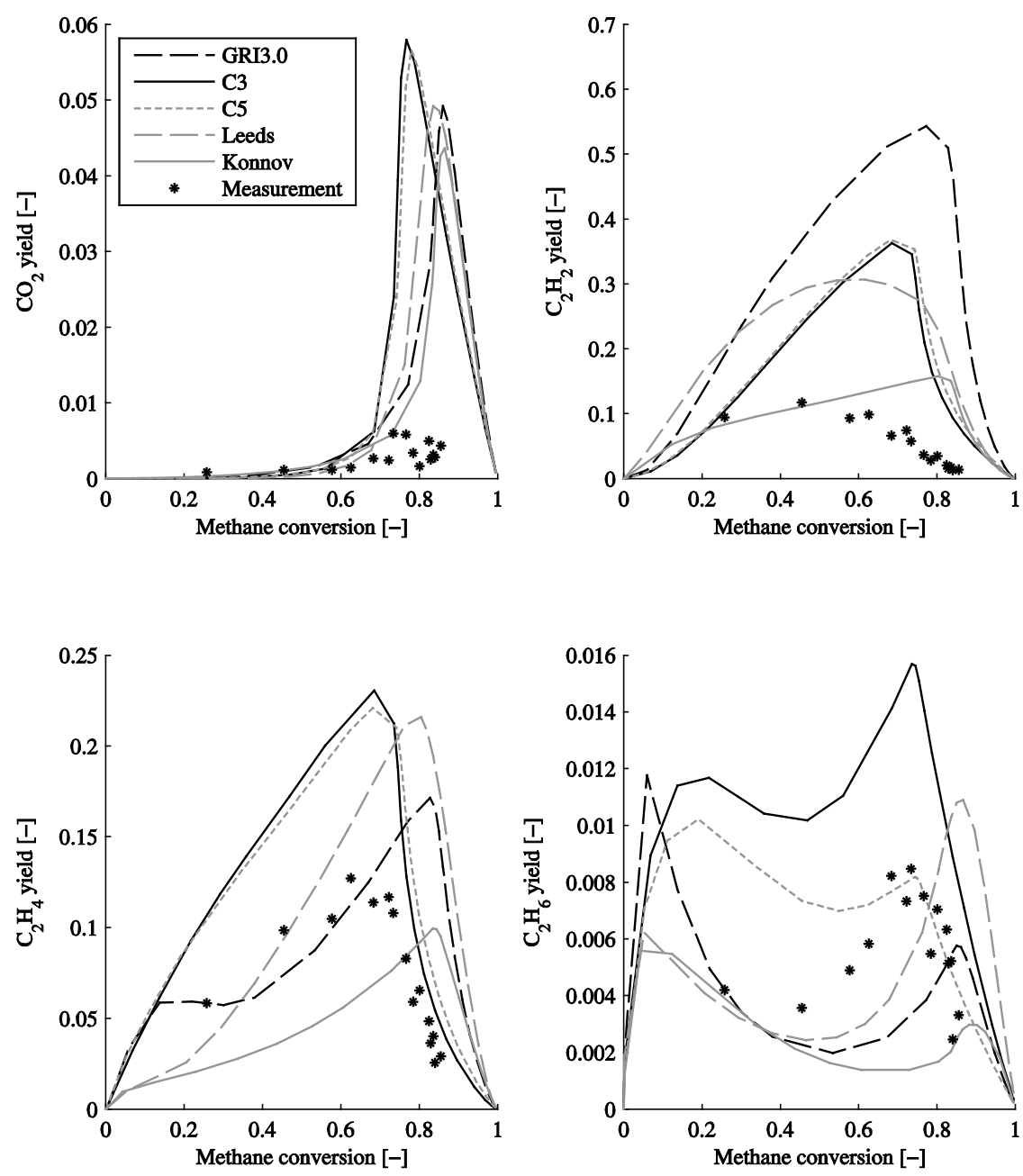

Figure 5.39, Minor species yield by simulations with mixture 4, initial temperature $543 \mathrm{~K}$ : $\mathrm{CO}_{2}$ (top left), $\mathrm{C}_{2} \mathrm{H}_{2}$ (top right), $\mathrm{C}_{2} \mathrm{H}_{4}$ (bottom left) and $\mathrm{C}_{2} \mathrm{H}_{6}$ (bottom), compared to experiment

\section{Hydrogen - carbon monoxide ratio}

Since the operating window of the steam reforming process in the PCR contains a part where hydrogen is formed without the formation of carbon monoxide, the hydrogen carbon monoxide ratio is very high in this region. This is shown in Figure 5.40, where the hydrogen carbon monoxide ratio is plotted with a logarithmic $y$-axis and only the part of the results from $40 \%$ methane conversion and upwards is shown. 

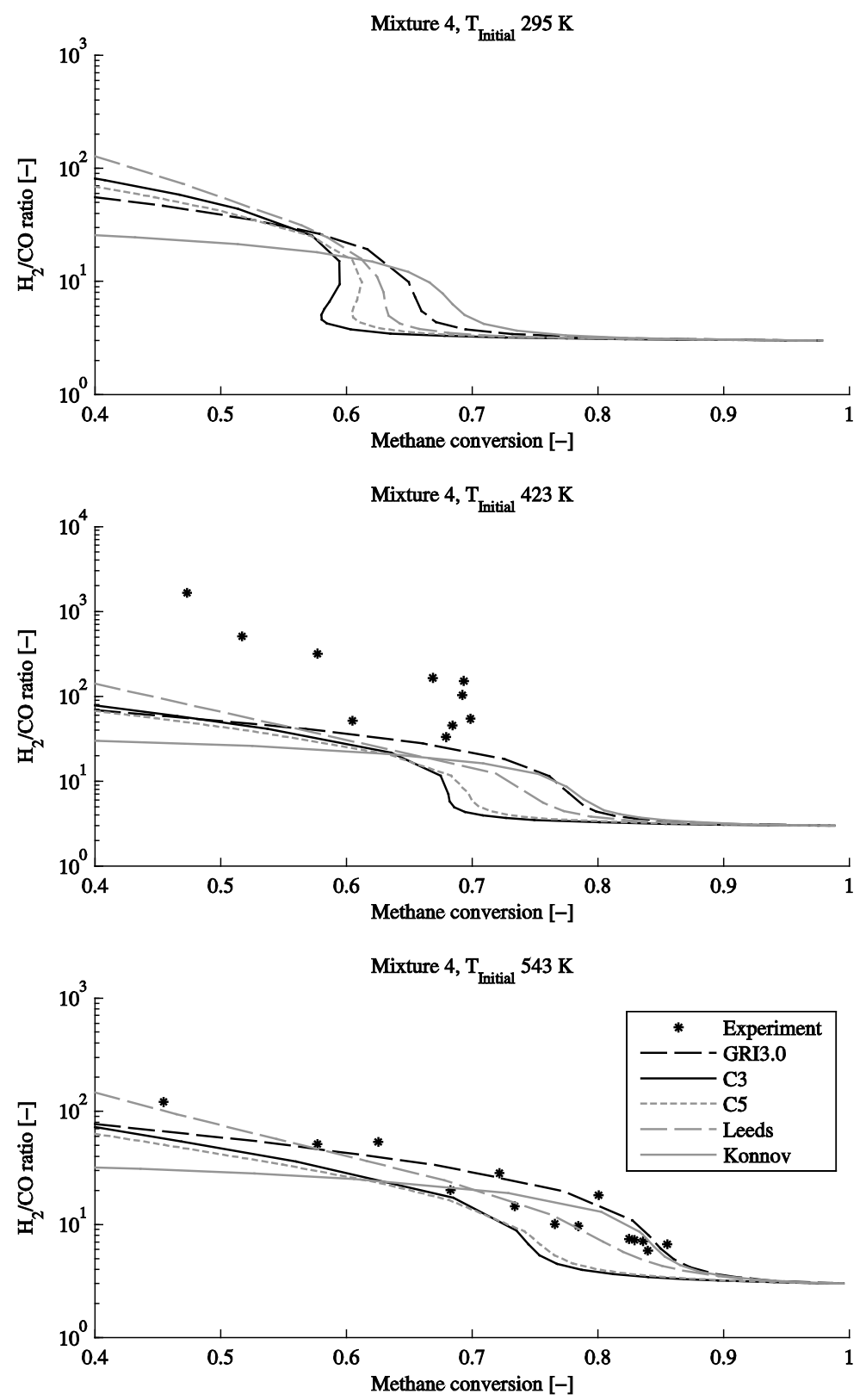

Figure 5.40, Hydrogen - carbon monoxide ratio, for mixture 4, initial temperature $293 \mathrm{~K}$ (top), $423 \mathrm{~K}$ (middle) and $543 \mathrm{~K}$ (bottom), compared to simulations 
The results of the $543 \mathrm{~K}$ initial temperature series show good correspondence with the models, while the $423 \mathrm{~K}$ initial temperature series deviates significantly. This is most likely caused by the increased amounts of soot that are formed at lower reaction temperatures, influencing the carbon monoxide yield more severely in the $423 \mathrm{~K}$ initial temperature series.

\subsubsection{The steam reforming process}

As with the partial oxidation process, the simulations can be used to take a look inside the compression expansion cycle. In Figure 5.41 the evolution of the major species during one compression expansion cycle is shown, simulated by the GRI3.0 mechanism. Here again it is evident that first methane is pyrolised, without effecting the steam concentration and that the formation of carbon monoxide, paired with the disappearance of steam, happens later in the cycle.

The time scale of the processes that happen in the steam reforming cycle is much longer than in the partial oxidation cycle, even though a comparable amount of energy is supplied. While the timescale of the partial oxidation cycle is in $10^{-4} \mathrm{~s}$, the timescale of the steam reforming process is expressed in $10^{-3} \mathrm{~s}$.

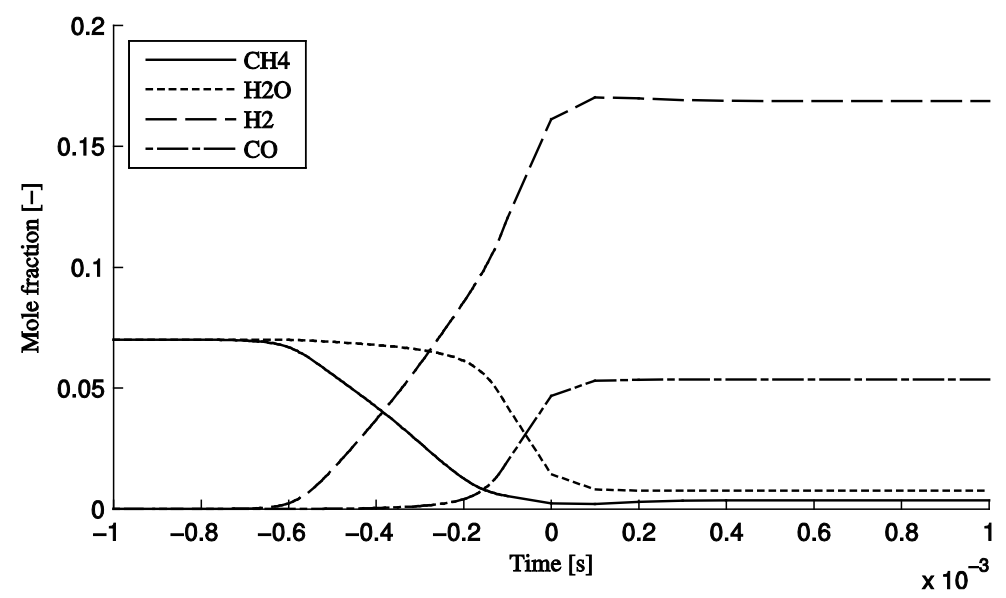

Figure 5.41, Major species compression expansion cycle with maximum piston kinetic energy $350 \mathrm{~J}$, simulated by the GRI3.0 mechanism. Initial temperature $543 \mathrm{~K}$

In Figure 5.42 the concentrations of the different side products, as simulated by the GRI3.0 mechanism are shown. In this plot the formation in the order, as predicted by the Kassel mechanism can be seen again. The first minor specie to form is ethane, followed by ethylene and finally acetylene. All species are predicted to drop to much lower 
concentrations again as the process continues. The carbon dioxide forms just before top dead centre and decreases somewhat near towards the end of the cycle.

Based on the concentrations of the minor species found, one could debate the accuracy of the term "minor species" concentrations and yields are so high that, for some of the process conditions the term "minor" does not entirely do the products justice.

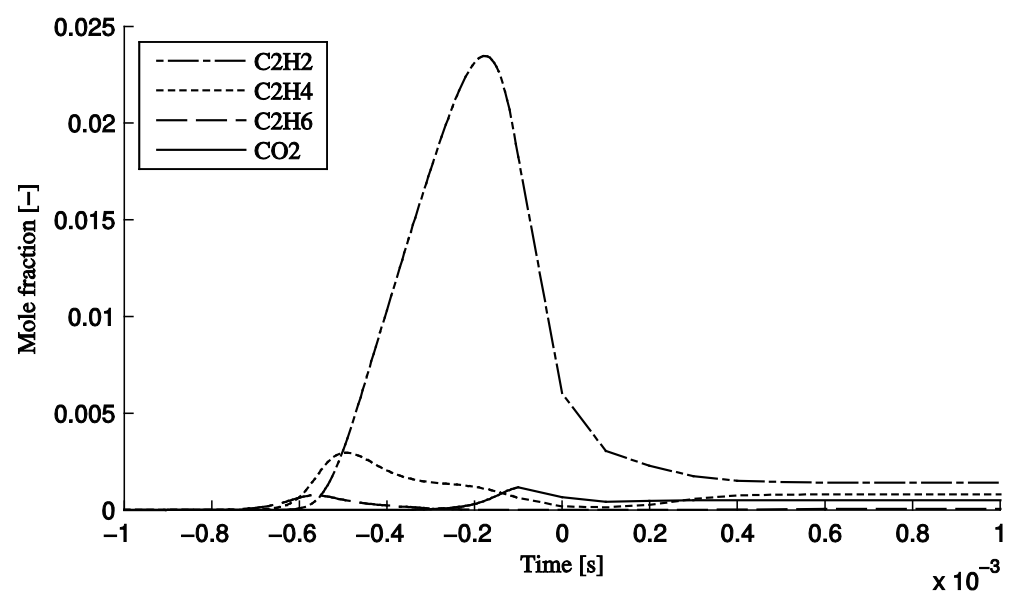

Figure 5.42, Minor species compression expansion cycle with maximum piston kinetic energy $350 \mathrm{~J}$, simulated by the GRI3.0 mechanism. Initial temperature $543 \mathrm{~K}$

For comparison purposes, the evolution of the major species during the same cycle, but simulated by the C3 mechanism is shown in Figure 5.43. While the same amount of energy is supplied in the simulations, the C3 mechanism predicts that steam starts disappearing from the mixture earlier in the cycle and that almost all steam present in the mixture is finally consumed. This is the only big difference between the two models, the formation of hydrogen, and final hydrogen yield is essentially the same. 


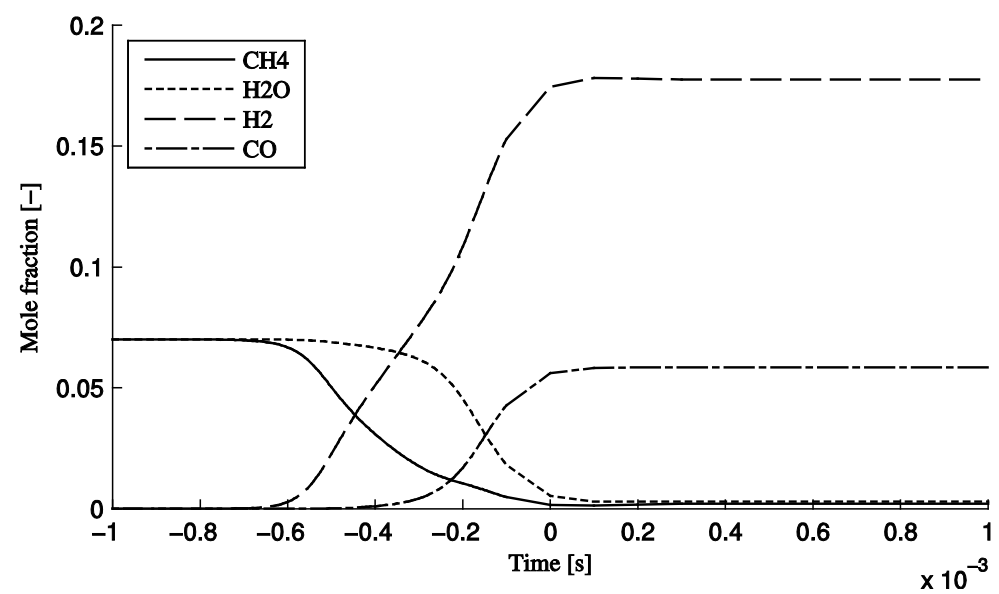

Figure 5.43, Major species and carbon dioxide during compression expansion cycle with maximum piston kinetic energy $350 \mathrm{~J}$, simulated by the C3 mechanism. Initial temperature $543 \mathrm{~K}$

The minor species concentrations predicted by the C3 simulation, shown in Figure 5.44, show great similarity with the predicted concentrations by the GRI3.0 mechanism. The only real difference is in the final concentration of carbon dioxide, the $\mathrm{C} 3$ model predicts that more carbon dioxide is used up during the rest of the cycle, after it has been formed.

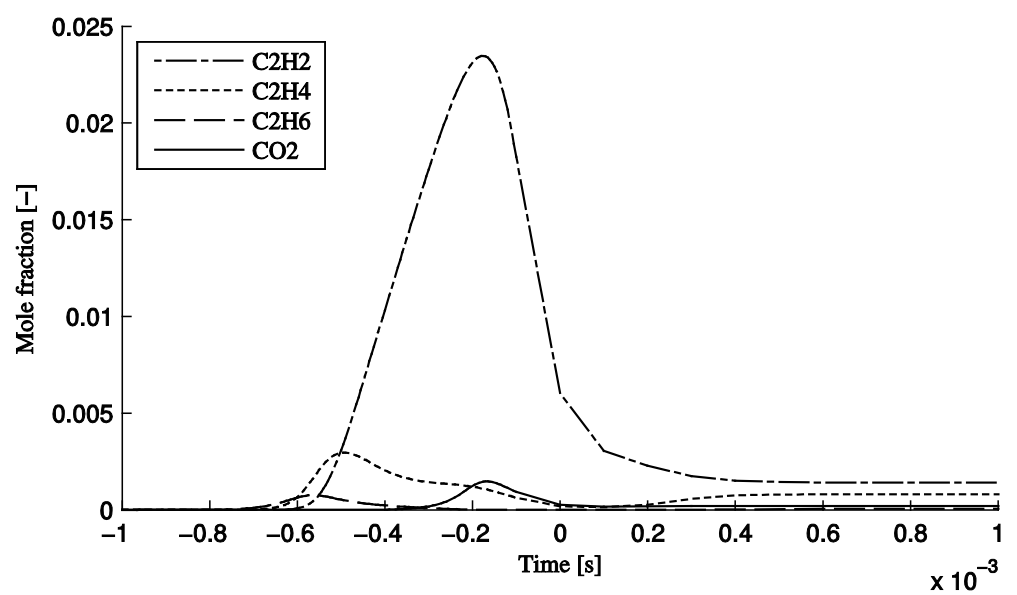

Figure 5.44, Minor species and carbon dioxide during compression expansion cycle with maximum piston kinetic energy $350 \mathrm{~J}$, simulated by the C3 mechanism. Initial temperature $543 \mathrm{~K}$ 



\title{
6 possibility of the PCR
}

\author{
One of the key issues of the continuous PCR is the matter of control. Based on the \\ experiments performed, both on losses and chemistry and the simulations done, a theory \\ has been developed that describes the behavior of the PCR in continuous operation mode. \\ This chapter describes the measurement and experimental results that were used to \\ arrive at this theory, as well as the theory itself.
}

\subsection{Introduction}

The functioning of the PCR can be compared to that of free piston engines, with some important differences. Most importantly there is the absence of a load on the piston. Though the pistons in free piston engines are referred to as "free" some degree of control through the load can be obtained. By increasing the load, more energy is removed from the system, which decreases the maximum piston speed, compression ratio and operating frequency. Alternatively, by decreasing the load, more energy is allowed to remain in the system, thus increasing the attained maximum piston speed, compression ratio and operating frequency. This is not the case for the PCR, since no load is present.

In the field of free piston engines different types are distinguished according to Mikalsen et al. [32]: the single piston type, the dual piston type and the opposed piston type. No matter which type of free piston engine is chosen, research done with any of the types all report on the importance of control of the combustion timing and process. Small variations in the combustion process, according to Aichelmayr [71] and Achten [34] have a large influence on the next compression. The reason for this being that the combustion process is, combined with the energy stored in the compressed gas, the driving force behind the next compression. The result is that when designs for a free piston engine are made, much effort is put into optimizing the amount of control on the piston motion, one of the factors that determine the combustion process. Mostly this is done by applying a controlled load to the piston. More sophisticated is the reported pulse pause modulation scheme for hydraulic free piston engines as put forth by Achten et al. [72]. 
The most important difference between the PCR and the research done towards free piston engines is the absence of a load. This means that the most important parameter available for control of the reaction process is not present. For this reason it is important to look at how the free piston reactor will behave, since the reaction process determines the final products. Also it is vital to know in advance whether the risk of run-away, where the PCR goes out of control and running at higher and higher frequency until it overspeeds to a point where it destroys itself due to mechanical failure, needs to be considered. These considerations are especially important for processes where energy is released during chemical transformations, since this is the area with the largest risk of run-away. For this reason the theory presented here focuses on the application of the PCR for exothermic application.

\subsection{The determining relations}

In the absence of load controllability, there are many factors which remain that influence the combustion process. Some of these are: the temperature of the reactor and piston surfaces, the kinetic energy put into the piston to initiate the combustion process, the type of reactants, their concentrations, initial temperature and pressure and the degree of mixing. In this analysis it is assumed that the temperature of the reactor and piston surface have reached a steady state and no longer change on a stroke-to-stroke basis. Also it is assumed that the feed of reactants (composition, mixing, temperature and pressure) does not change on a stroke-to-stroke basis.

To analyze the problem of controllability and run-away, under this assumption of all else remaining equal, two relations can be identified:

- The relation between energy input and ignition moment

- The relation between ignition moment and change of kinetic energy of the piston

These relations alternate one after another in a continuous process. The first relation determines the connection between the initial kinetic energy of the piston and the moment of auto-ignition of the reactive mixture in the reaction chamber. The second determines the connection between the moment of auto-ignition and the energy that is added to (or removed from) the piston during the cycle. This energy change leads to a new initial kinetic energy of the piston for the next cycle, in turn leading back to the first relation of auto-ignition moment. 
If the characteristics of each of the relations are known, the entire continuous process, including controllability and run-away properties can be described. To determine the characteristics of each of the relations, two sources of data are used. Firstly experimental data from the single shot reactor, described in section 0 , is used. Data from this reactor is compared to and complemented by the second source of data, which are the simulations of the reactor. The simulation method was previously described in section 5.7 .

\subsection{The relation between energy input and ignition moment}

The first step when looking at the controllability and run-away propensity of the PCR is finding a relation between the energy input and the ignition moment of the reactants. For this purpose the experimental results are used. In Figure 6.1 a series of measurements is shown, with the same mixture in the reactor $\left(10\right.$ vol.\% $\mathrm{CH}_{4}, 5$ vol. $\% \mathrm{O}_{2}$, 20 vol.\% $\mathrm{N}_{2}$ and 65 vol.\% $\mathrm{Ar}$ ) and same starting conditions (423 $\mathrm{K}$ and 1 bar), but with different energy input into the piston. In these measurements the estimated ignition moment, based on the pressure trace, has been indicated. It can be seen that for the lowest energy input in this series (maximum piston velocity $17.6 \mathrm{~m} \cdot \mathrm{s}^{-1}$ ) no ignition of the mixture in the reactor takes place. The pressure curve simply shows a smooth compression expansion cycle, without any humps caused by ignition. As the energy input is increased, the measurement with the maximum piston velocity of $19.6 \mathrm{~m} \cdot \mathrm{s}^{-1}$ shows ignition just after the piston reached top dead centre (TDC). As the energy input is increased stepwise again and again, the ignition moment moves forward relative to the cycle, igniting around and even before the TDC. The oscillating pressure signal after the initial ignition moment can most likely be attributed to the characteristics of the pressure sensor and its mounting method and the reaction kinetics, but this will not further be discussed in this chapter. 


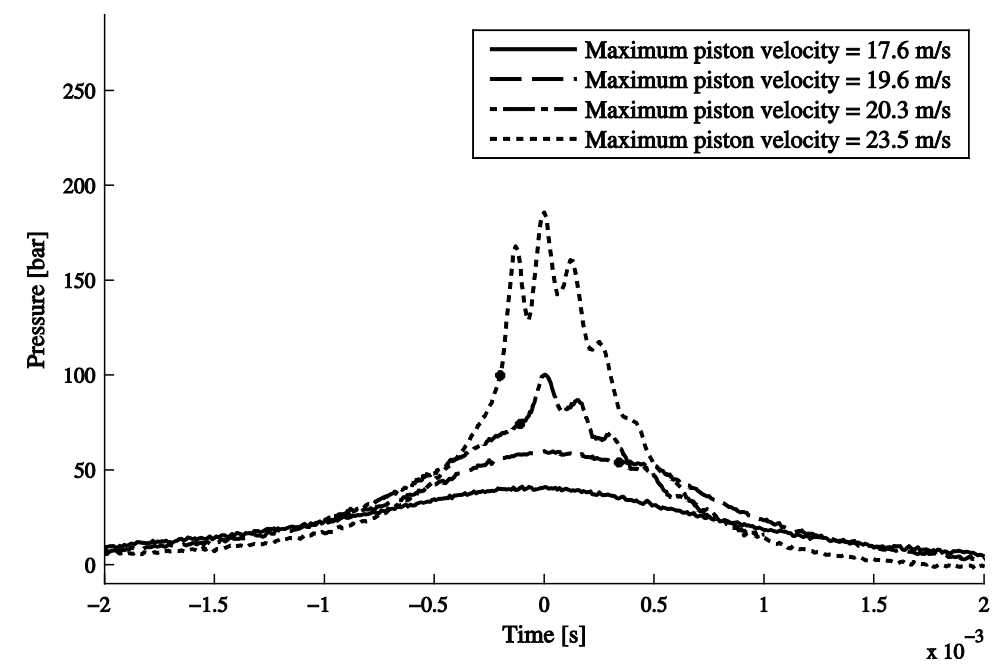

Figure 6.1, Pressure traces of four experiments with different maximum piston velocities

The experimental setup does not allow for exact quantitative determination of the ignition moment. However, the simulations done of the same process do. When the same process as shown in Figure 6.1 is simulated a graph as shown in Figure 6.2 can be constructed. In this graph, the ignition moment is plotted against the maximum piston velocity. More points were simulated than the four example experiments shown in Figure 6.1, within similar bounds.

To construct this graph several definitions were needed. Firstly, the ignition moment is defined in terms of a "dimensionless time number". A dimensionless time number of 0 is defined as ignition at TDC and an ignition moment of -1 as ignition immediately at the beginning of the cycle. This means that the cycle ends at a dimensionless time number around 1 (slightly more than 1 if the downward part of the cycle is longer than the upward part and slightly less than one if the downward part of the cycle is shorter than the upward part). The moment of TDC is taken from the simulated piston path. The second definition that is needed is the definition of "ignition". This is defined as follows: the pressure trace of the reacting simulation was compared to the pressure trace of an identical simulation where the reactions were disabled in the software. A criterion could then be applied, in terms of difference between the two pressure traces, to indicate the moment of ignition. In Figure 6.2 two different criteria for ignition are shown: the criterion where the pressure data from the non-reacting simulation differs $10 \%$ from the reacting simulation, and the same for a $5 \%$ difference. While the two lines that appear 
differ somewhat (a larger difference criterion means the moment of ignition is later), the same shape of curve is visible.

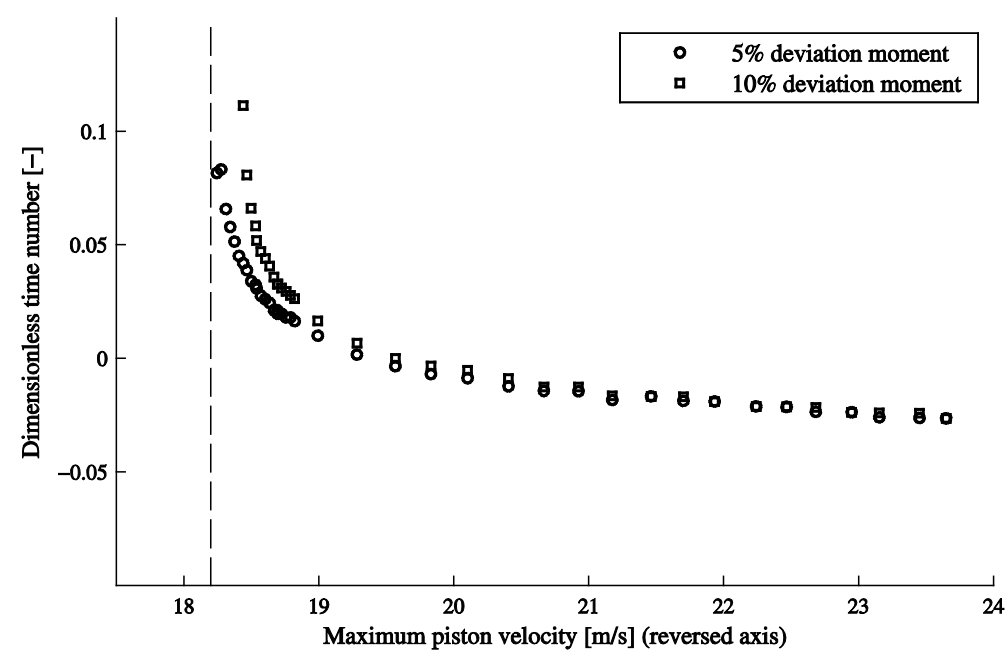

Figure 6.2, Ignition moment as a function of piston energy

The Figure 6.2 shows the relation between energy input and dimensionless time number that was being sought. Ignition is not possible below a certain threshold energy input. Naturally the exact position of threshold depends on the definition of what constitutes ignition. In the case of the conditions of the experiments and simulations done, and the definitions as used in this chapter the threshold is around $18.2 \mathrm{~m} \cdot \mathrm{s}^{-1}$ maximum piston velocity. This is illustrated by the vertical dashed line in Figure 6.2. This means that, if the maximum piston velocity is less than $18.2 \mathrm{~m} \cdot \mathrm{s}^{-1}$, no ignition will take place.

The minimum energy required for ignition to take place is from here on referred to as "threshold energy". A second important realization that can be made is that, as energy input is increased, ignition can never be forwarded beyond a certain dimensionless time number. While it is not immediately clear from the simulations where this limit lies (the line formed by the simulated points has not become completely horizontal), it is clear that as the energy input is increased dimensionless time numbers of -1 and 0 will come closer together in time. The ignition moment will always remain somewhere between the two, no matter how much energy is given to the piston. The earliest ignition possible is from here on referred to as "critical ignition moment". 


\subsection{The relation between ignition moment and change of kinetic energy of the piston}

The second of the relations introduced in section 6.2 is the relationship between ignition moment and energy change. For this relation, results can again be obtained from both experiments and simulation. However, in this case the difference between the two results is larger. The reason is that the order of magnitude of the energy change are, as follows from estimates of the energy losses, for the conditions used in this article, the same as (or close to) the order of magnitude of the energy losses from the reaction chamber (as can be seen in section 4.4.1). This means that, for any exothermic reaction simulated, no matter how little energy is released, this will always result in a positive energy change of the piston. In the experiments on the other hand, the energy released is also used to compensate for energy losses. So a significant energy release is required for the piston to experience an increase in energy. If the energy release is not high enough, or badly timed, the energy input will not overcome the energy losses and the piston will experience a negative energy change, something that could not happen in the simulations. This is shown in Figure 6.3 and Figure 6.4. In these figures, the difference in squared maximum velocity between the up and down stroke is used as a measure for the energy change of the piston. To make the two figures more comparable the $x$-axis in Figure 6.4 has been reversed. By doing so, experimental data that corresponds to ignition before TDC is shown on the left hand side and data that corresponds to ignition after TDC on the right hand side: the same as in Figure 6.4.

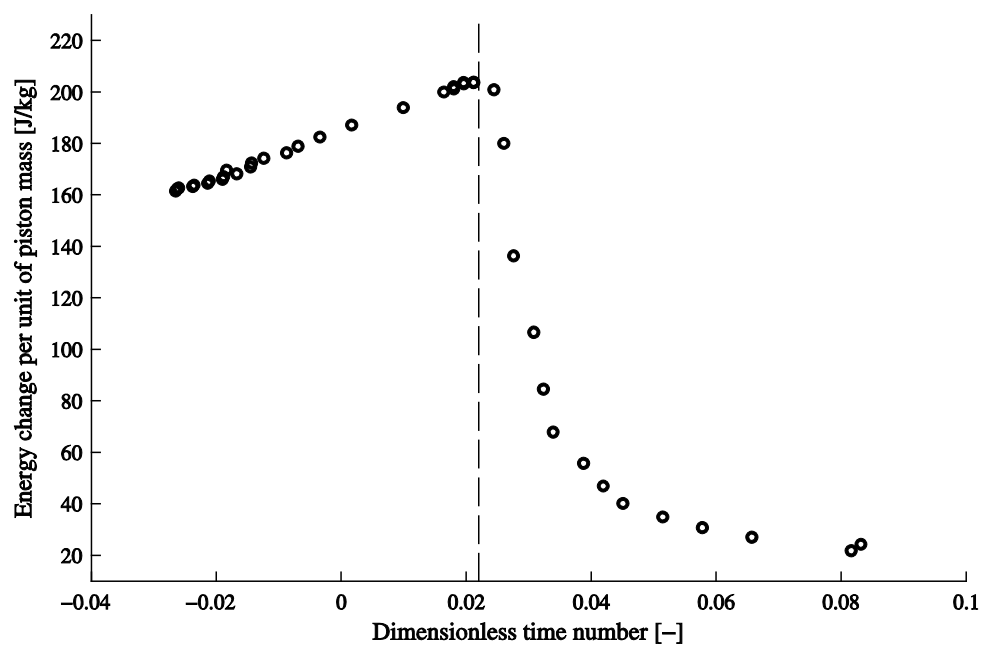

Figure 6.3, Energy change as a function of ignition moment, determined by simulation 


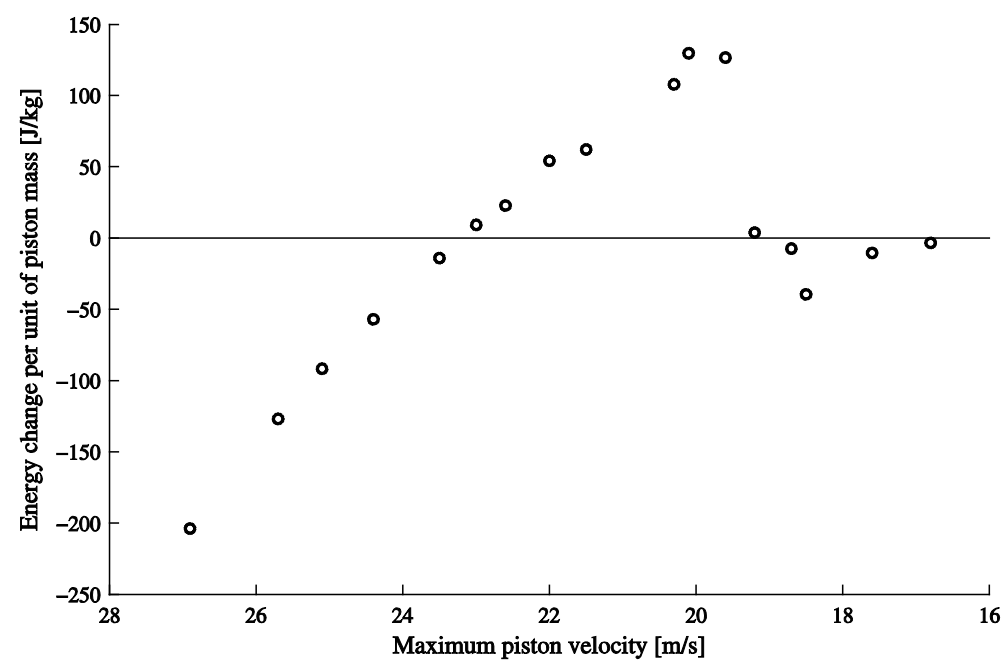

Figure 6.4, Energy change as a function of maximum piston velocity, determined experimentally. Note that the $x$-axis is shown reversed.

In Figure 6.3 it is visible that the energy change of the piston in the simulations is always positive and that the energy change of the piston depends on the ignition moment. The reason is that the energy release by the chemical reactions depends on the ignition moment: as ignition is earlier, more time remains for endothermic reforming steps. As ignition is later, not the entire combustion step is completed. Thus there is an optimum moment of ignition for the most energy to be transferred to the piston. For an instantaneously complete reaction this moment would be exactly at TDC, but for the situation shown here this moment is approximately $2.2 \%$ after TDC, as indicated by the vertical dashed line. This same trend is seen for the experiments. In the case of the experiments the energy change is positive at the optimum ignition moment, but decreases and is finally even negative as ignition takes place earlier. The rate of decrease in energy change is much larger, which can be explained by looking at the energy losses: to get a lower dimensionless time number, more energy needs to be supplied, peak temperatures and pressures are higher, the period with high losses is longer and so, losses also increase. This decreases the energy change drastically.

If ignition takes place after the optimum moment the energy change of the piston is also recorded to be less than the maximum, in the case for the situation shown going to around zero. 


\subsection{The controllability}

Now that both relations that determine the controllability of the PCR have been mapped, they can be combined to reach a conclusion. The energy change as a result of the ignition event (or absence thereof) can be added to the energy of the piston. This is schematically shown in Figure 6.5. In this figure, the solid line represents the energy that was put into the piston at the beginning of the cycle (with the energy shown on the $y$ axis). As was shown, each of the piston energy levels corresponds to a unique ignition moment, which in turn corresponds to a change in piston energy. The new energy that the piston has for the next stroke (the sum of its initial energy and the energy change) is shown by the dashed line. The original energy line and new energy line have two intersections, corresponding to the operating points where the energy change is zero. These will be referred to as the stable operating point with corresponding stable operating energy (the leftmost intersection) and the unstable operating point with corresponding unstable operating energy (the rightmost intersection).

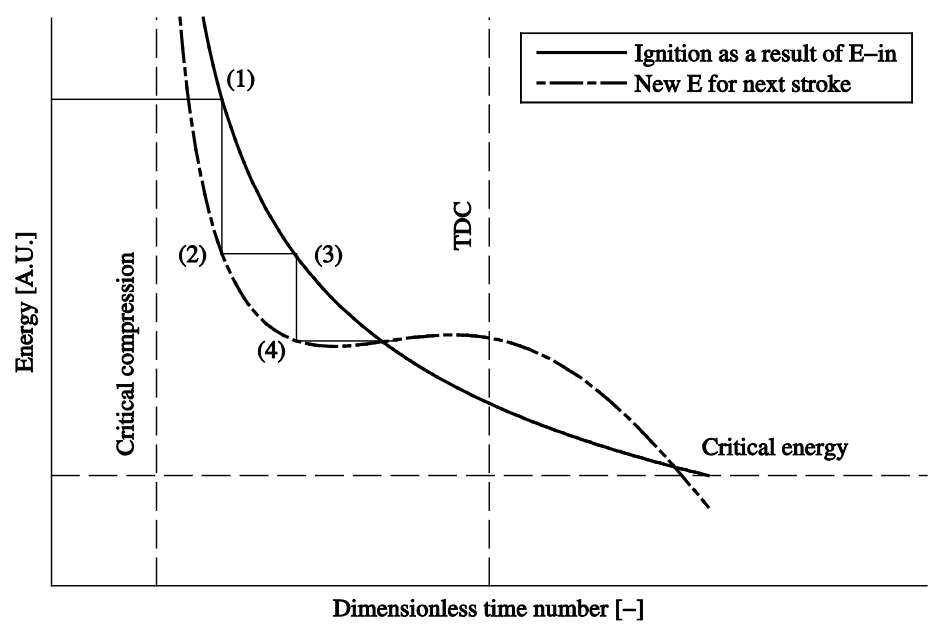

Figure 6.5, A schematic illustration of the two relations that determine controllability and run-away and the convergence of the process to steady operation

The operation of the reactor can now be described as follows. To start the process, the piston is given an amount of energy, more than the critical and stable operating energy, as indicated by (1) in Figure 6.5. Then, on the solid curve the ignition moment can be found. As a result of the ignition at this moment, for the next cycle, the piston will have less energy, as indicated by point (2) on the dashed curve. The ignition moment of the next cycle can then be found again on the solid curve, indicated by point (3), and for the 
next cycle the energy of point (4) will be available. As this process continues the piston energy converges to the stable operating point, until it no longer changes cycle-to-cycle.

Similarly one might start the process by giving the piston an amount of energy between the stable operating energy and the unstable operating energy. From this moment, the process will also converge to the stable operating point. If less energy than the unstable operating energy is given to the piston, the process will diverge away from the stable operating point, until after a number of cycles no ignition takes place at all, and the process stops. Hypothetically, one might add exactly the unstable operating energy and the process might run at the unstable operating point. However, a tiny change in process conditions will cause the process to either shift to the stable operating point, or stop all together. This makes operation at the unstable operating point unrealistic. The data presented in this article suggests that, for the conditions investigated, the stable operating point is located at a piston kinetic energy corresponding to a maximum velocity of around $23 \mathrm{~m} \cdot \mathrm{s}^{-1}$.

The curves and theory presented in this article makes some important assumptions about various parameters. For example, for some mixtures the unstable operating point may not exist, or even the stable operating point. Also, ignition before TDC may cause such a tremendous energy loss that the resulting energy for the next stroke is below the stable operating energy or even below the unstable operating energy. If this is the case, one might supply less energy to start the process. There are many situations imaginable where no stable operating energy will be found and the process will not be sustainable, but the approach presented here can be a powerful tool for understanding and optimizing the process.

\subsection{Conclusions}

The theory and experimental results show that for certain specific reactant flows into the PCR the process will converge to a stable operating point, as long as more energy is supplied than the unstable operating energy. Other parameters that influence the shape of this curve, such as reactor and reactant temperature, piston mass, reactor design, chemical energy content of the reactant stream and many others, will naturally change the shape of the curves presented in this chapter, but the basic idea stays the same. This means that one might add a third axis to the curve in Figure 6.5, representing a third parameter to be changed. For example, one might add a third axis with the reactant initial temperature, and create two 3-D surfaces, with the stable operating energies for different initial reactant temperature at their intersections. 
The approach presented gives clear insight in the controllability of the PCR operation: operation will either converge to a constant stable operating point, or cease. Though this might lead one to conclude that any process that has energy losses will be inherently stable and never run away, this depends heavily on the definition of run-away used. As energy losses are reduced, the stable operating point will move to a higher energy level. So, hypothetically, in a reactor with very little energy losses the process might be considered to run away (depending on the definition). 


\section{Conclusions and Recommendations}

In the first chapter of this thesis several research questions were posed. The research presented in this thesis is an attempt to find answers to these questions. The first item that is discussed in this concluding chapter is the answers to these research questions.

The research done within the scope of this project, both experimentally and numerically, has yielded many conclusions. These were in many cases mentioned when the results were presented. In this chapter the most important conclusions that were drawn from the experiments and simulations done are summed up and clarified. They are sorted in the same order as they have been introduced in the preceding chapters.

The conclusions are followed by various recommendations, both for improving and deepening the results obtained as well as suggestions for follow up research.

\subsection{Answering the research question}

In the introductory chapter, three research questions were posed. These were:

1. How large are the losses from a rapidly compressed and expanded volume of gas, and how do these losses compare to energy contained in the compressed gas?

2. Can stable operation with a completely free piston as it is intended with the PCR be achieved?

3. Will rapidly compressing a mixture of methane and oxygen, or methane and steam, yield synthesis gas? And if it does, how will the yields of syngas compare to conventional reactors?

In short, the answers are:

1. For relatively low compression ratios the steady state heat transfer coefficient was found to be between 20 and $100 \mathrm{~W} \cdot \mathrm{M}^{-2} \cdot \mathrm{K}^{-1}$. In this particular reactor in the order of $1.2 \%$ of the energy contained in the compressed gas was lost per cycle. 
For high compression ratios only the heat loss in the first compression expansion stroke was calculated. In this first stroke, the heat loss was $36 \%$ of the maximum work done on the gas.

2. Yes. When the energy released by chemical reactions is large enough to compensate the losses from the reactor at some operating point, operation of the reactor will converge to that operating point.

3. Yes, yields of hydrogen can be as high as $83 \%$ with partial oxidation and as high as $115 \%$ with steam reforming. The yield of carbon monoxide can be as high as $87 \%$ with partial oxidation and $35 \%$ with steam reforming. This shows that, theoretically, the PCR can have an overall carbon efficiency of well over $85 \%$. Partial oxidation yields are significantly higher than the adiabatic, isobaric equilibrium concentrations.

In addition to answering the research question, many other conclusions can be drawn. These will be discussed in the following sections.

\subsection{Conclusions on the setup}

Within the scope of the research presented in this report, two setups were used. The first one is referred to as the continuous setup, the second one as the single shot setup. The conclusions that can be drawn on the functioning and design of the setups will be discussed separately for each setup.

\subsubsection{The continuous setup}

Operating a continuous PCR, without any control over the temperature, in driven single chamber mode turned out to be quite complicated. While the reactor operated well for driving pressures below 6 bar, where the temperature change of the various parts was less than 18 degrees, operation quickly became instable at higher operation pressures. The temperature change that was caused by the experiment made that the change in the gap size between piston and cylinder became large enough to influence the operation of the reactor.

The result of these problems was that only relatively short runs could be achieved at higher driving pressures. While the obtained data is still valuable, a great improvement could be made by solving the gap-size problem. 


\subsubsection{The single shot setup}

The conclusions on the single shot setup can be summarized in the following points, which will be further explained in the subsequent sections.

- Achievement of a unique operating window of pressure and temperature.

- True single shot behavior is not achieved, but the motion is significantly damped.

- Experiments are consistent and repeatable.

- Control over the various process parameters is high enough to yield useful results.

- Sensors are, in general, adequate for the purposes for which they were employed, but have visibly reached their limits.

- Maintenance of the setup was reasonable. Parts that were expected to have a large amount of wear were replaced after a reasonable number of experiments.

\section{Achievement of a unique operating window of pressure and temperature}

The single shot setup used is a unique machine, capable of reaching a high pressure and temperature operating window that cannot be reached by conventional reactors or even conventional ballistic compressors. Pressures were as high as 700 bar with the light piston and well beyond 1000 bar for the heavy piston. Corresponding adiabatic temperatures were, if calculated by ideal gas relations well beyond $2000 \mathrm{~K}$, with compression ratios of 30 reachable.

\section{Single shot behavior}

True single shot behavior was not achieved with the setup, but the damping was adequate enough to reduce the compression pressure of the second compression stroke to roughly half of the first compression. This is considered to be enough to be able to conclude that all processes of interest happen in the first compression cycle. This could off course be verified by modeling the second reciprocation in the numerical simulation model developed.

\section{Consistent and repeatable experiments}

The experiments that were performed in the single shot setup were well-nigh perfectly repeatable. When the same settings were used in the launch mechanism and control board, the measured pressure trace and piston velocity varied by no more than a few 
percent. This was well low enough to be able to double check results and have confidence in the validity of the results obtained.

\section{Control over process parameters}

As mentioned, using identical launch settings yielded nearly the same results. The same applied to small variations in the launch settings. These yielded controllable variations in the peak pressure and peak piston velocity.

The method of preheating the reactor enabled good control over the initial temperature, with variations in the initial temperature smaller than half a degree. The result was that neat curves of a single varying parameter could be constructed.

The only aspect that might be improved is the control over the initial mixture. The mixtures were ordered with a mixing accuracy of $10 \%$ relative, but this was not always what was received. Of course this did not influence the results in a disastrous manner, since the mixtures could be analyzed by gas chromatograph prior to use in experiments.

\section{Sensor performance}

The different sensors were adequate for the purpose for which they were used, but reached their limits during the experiments. The pressure sensor in the reaction chamber had a limit of 700 bar and was rated to be able to operate at a maximum temperature of $300{ }^{\circ} \mathrm{C}$, but did, under severe experiments, show great irregularities in the measured signal. Also, the pressure reached in the experiments with the heavy piston was well beyond 700 bar.

The velocity sensor made use of modulated light with a frequency of $30 \mathrm{kHz}$, meaning it could never measure at a higher rate than $30 \mathrm{kHz}$. This was slower than the rate required to measure the passing of the grooves in the piston as a distinct event. This was solved by using a reasonably complicated data analysis method, but certainly higher piston velocities than those occurring in the presented experiments would not be measureable.

The temperature sensor was certainly fast enough to cope with the speed of the experiment, but the data generated required a complex analysis method, that made results less reliable. Though the sensor yielded useful results in this case, the sensor cannot be used in chemical reacting cases. Also the robustness of the sensor left something to be desired. Experiments with launch pressures above 70 bar generally destroyed the junctions formed over the mica layer, indicating that a more robust sensor may be more suitable for use in the PCR. 
A Gas Chromatograph is a reasonably simple machine, which can be very complicated to operate. The operation of a GC is sometimes referred to as an "art" and this is quite a fitting description. An aspects that can strongly affect accuracy of the GC, next to a skilled operator, is high quality calibration gas. The quality of the calibration gasses was sufficient for accurate measurement of all species, with the exception of ethylene and acetylene. The quality of the calibration gas used for these species was not as high as it should have been.

\section{Maintenance of the setup}

During the course of this project no maintenance other than the occasional cleaning was needed for the reactor and launch mechanism. Some valves in the control system had to be replaced, which was to be expected.

\subsection{Heat transfer}

\subsubsection{Average heat transfer}

The experiments on averaged heat transfer, followed by the outlined data analysis method, yielded an empirical relation between the peak pressure in the continuously operated reactor and the maximum heat transfer coefficient:

$$
h=0.18 \cdot P^{1.78}+21.9 \text {. }
$$

In this relation the peak pressure is in bar and the heat transfer coefficient in $\mathrm{W} \cdot \mathrm{m}^{-2} \cdot \mathrm{K}^{-1}$. The form of this function is chosen purely for convenience reasons, it is not implied that the pressure is in fact the only determining factor that determines the heat transfer coefficient. This relation only applies to the reactor used. If a reactor with a different geometry, compressing a different gas or operating in a different compression regime, is used, the empirical relation may not apply.

The found heat transfer coefficient ranges from 25 to $100 \mathrm{~W} \cdot \mathrm{m}^{-2} \cdot \mathrm{K}^{-1}$. This is in the same order of magnitude as the heat transfer coefficient for forced convection situations. However, the definition of the heat transfer coefficient used the highest possible $\Delta T$, so in reality the actual heat transfer coefficient will be somewhat higher, depending on the definition used.

The results were also used to compare the average heat lost in this particular operating case of this particular PCR, to the work done on the gas every cycle. It was concluded that $1.2 \%$ of the work done on the piston was lost to the walls in the form of heat. Of 
course, in reality, many factors influence the heat loss from the reactor, and in reality the heat lost may be much lower than the calculated value for this particular case.

\subsubsection{Instantaneous losses}

The experiments on instantaneous losses, with the following data analysis method yielded an empirical relation between the pressure in the reactor and the heat flux from the reactor:

$$
q=P^{3.08}+8.0 \cdot 10^{4} \cdot P .
$$

In this relation the pressure is in bar, and the heat flux in $\mathrm{W} \cdot \mathrm{m}^{-2}$. The form of this function chosen purely for convenience reasons, it is not implied that the pressure is in fact the only determining factor that determines the heat flux. The relation only applies to the reactor used. If a reactor with a different geometry, parts made from different materials, compressing a different gas or operating in a different compression regime (initial temperature, compression ratio, initial pressure), is used, the empirical relation does not apply.

The relation is based on data between 0 and 350 bar and gives the heat that is lost during the first compression expansion stroke. This means that if this relation is used to calculate the heat loss of a cycle, an upper bound of the heat loss will be found, since most heat is lost during the first cycle. Using the relation found, the heat loss during the first compression expansion cycle with a peak pressure of 120 bar is found to be $36 \%$ of the work done on the gas.

\subsubsection{Average and instantaneous losses compared}

The results obtained in the average heat loss measurements cannot be directly compared to the instantaneous heat loss because they were determined in different reactors. The stroke length, piston size and mass and cover geometry are different for the two reactors, which influences the found results. However, the order of magnitude of the losses found can be compared, and they are similar. The instantaneous heat losses between 0 and 20 bar vary between 0 and $1.6 \cdot 10^{6} \mathrm{~W} \cdot \mathrm{m}^{-2}$. The average heat loss when peak pressure is 20 bar is found to be $2.4 \cdot 10^{4} \mathrm{~W} \cdot \mathrm{m}^{-2}$. The fact that there is two orders of magnitude difference between the instantaneous heat loss and average heat loss is perfectly acceptable, since the peak pressures only occur during very short periods in the operation of the PCR, making the average heat loss much lower than the instantaneous heat loss. At the same time, the highest average heat loss measured was after between 50 and 400s, while the instantaneous heat flux is measured during the first reciprocation. 


\subsection{Chemistry in the PCR}

\subsubsection{Experiments on syngas formation by partial oxidation in the PCR}

In the investigation of syngas production by partial oxidation in the PCR the following parameters were investigated:

- Reactant composition

- Initial temperature

- Piston kinetic energy

- Piston mass

- $\quad$ Cover geometry

On each of the points conclusions were drawn, supplemented by a series of additional conclusions. Firstly the general conclusions on the chemistry will be discussed, then the specific conclusions on the parameters that were investigated.

\section{Partial oxidation chemistry}

From the results obtained it can be concluded that the partial oxidation of methane in the PCR can be seen as a process occurring in two steps. The first step is a combustion like step, which occurs very rapidly and results in a jump in yielded hydrogen and carbon monoxide as well as steam and carbon dioxide. This combustion like step is followed by a more slow process of methane cracking and steam reforming. In this step more hydrogen and carbon monoxide is formed, but also side products such as ethane, ethylene, acetylene and soot. Experiments show that there is a small gap in terms of energy that needs to be supplied between the partial oxidation step and steam reforming step. The size of this gap depends on the ratio of oxygen to methane in the reactants.

The order in which the side products are formed corresponds well to the well known Kassel mechanism. First ethane is formed, followed by ethylene, acetylene and finally soot. As the reaction approaches complete conversion, the different side products disappear.

Results show that the partial oxidation of methane is a very suitable process to be performed in the PCR, 95\% conversion of methane was achieved, with yields of hydrogen as high as $85 \%$ and yields of carbon monoxide close to $90 \%$. These results were obtained when the reactor temperature was $543 \mathrm{~K}$, which is a significant improvement over conventional chemical reactors. 


\section{Reactant composition}

A change in reactant composition influences the yield of some of the products, but not necessarily all. For example, a small increase in the oxygen concentration increases the carbon monoxide and carbon dioxide yield, but does not influence the hydrogen yield. This influences the hydrogen - carbon monoxide ratio significantly.

The presence of argon in the mixture increases the adiabatic temperature, resulting in less piston kinetic energy being needed to cause ignition. A similar mixture without argon gives identical yield curves, but shifted along the kinetic energy axis.

\section{Initial temperature}

A higher initial temperature means that less energy needs to be supplied by the piston to give the same result. This can be seen in the yield graphs of different products, which are shifted along the piston kinetic energy axis as the initial temperature is changed.

Comparing the yield of hydrogen to the total energy supplied (the sum of kinetic and thermal energy) it is found that supplying energy to the reactants in the form of kinetic energy of the piston is more efficient than supplying energy in the form of thermal energy prior to compression. On the other hand, if more piston kinetic energy needs to be supplied, a higher compression ratio will be achieved, in a colder reactor resulting in (at least initially) higher losses. So, there is likely an optimum operating temperature in terms of achieving high yields with minimal energy input.

Full conversion of methane by partial oxidation was not achieved from ambient initial temperature. However, the results obtained follow the same trends as results obtained with higher initial temperatures where near complete conversion was achieved. This leads to the conclusion that it is likely that, if a reactor is constructed where the maximum piston kinetic energy can be increased further, near complete conversion can also be achieved from ambient temperature and the formation of side products such as soot can be reduced to nearly zero.

\section{Piston kinetic energy}

The relation between the kinetic energy of the piston and the chemistry occurring in the PCR is very clear. Higher maximum piston kinetic energy allows the reaction to proceed further. This is in spite of the fact that if the piston kinetic energy is increased, cycle length decreases. Clearly, the increase in maximum temperature and pressure has a greater influence on the rate of the chemical kinetics, than is compensated for by the reduction in cycle length. 
The result is that as piston kinetic energy is increased, more methane is converted and more hydrogen and carbon monoxide is formed.

\section{Piston mass}

The most striking result of the heavier piston in the setup used is that the maximum piston kinetic energy of the heavier piston is much higher for the same launch pressure. This means that launching a heavy piston can be done much more efficiently: less high launch pressure is required to give the same result.

While cycle length is different for a different mass piston, there was no noticeable different in product yield, when the yield is plotted as a function of maximum piston kinetic energy. So, the heavier piston will give similar yields, at lower velocities. This means that the mass of the piston can be used manipulate parameters such as operating frequency, without paying the price of decreasing product yield.

\section{Cover geometry}

The different cover geometries tested did not give a change in product yield. This would lead one to conclude that either the influence of the walls on the chemistry is so small that a change in wall geometry does not lead to discernable changes in the product, or that the changes applied in the cover design are not large enough to influence the size of the adiabatic core in the reactor.

\subsubsection{Experiments on syngas formation by steam reforming in the PCR}

In the experiments performed to investigate the generation of syngas by steam reforming of methane, the following parameters were studied:

- Initial temperature

- $\quad$ Piston kinetic energy

On each of the points conclusions were drawn, supplemented by a series of additional conclusions. Firstly the general conclusions on the chemistry will be discussed, then the specific conclusions on the parameters that were investigated.

\section{Steam reforming chemistry}

The chemistry that occurs in the PCR when steam reforming is intended to occur can be divided into two main steps. These steps follow each other as the reactant mixture is compressed and their level of completion depends on the amount of energy that is supplied. The first step is the cracking of methane, yielding hydrogen, as well as ethane, ethylene, acetylene and soot (in that order). This cracking of methane is followed by the 
steam reforming step, yielding more hydrogen and carbon monoxide. However, at this point a lot of soot has already been formed, making pure steam reforming not a very attractive PCR process, when the process parameters as used in this research are upheld (unless soot is an intended product). While results with partial oxidation indicated that, as reactions approached full methane conversion, soot levels dropped to zero, it is unclear whether this will also be the case in the steam reforming process. It is however very likely changing the process parameters, such as increasing initial temperature or increasing the mole fraction steam, will greatly improve the success of pure steam reforming.

A side product of the experiments on steam reforming is that it was shown that cracking of methane is also very achievable in the PCR. These results were obtained when the reactor temperature was $423 \mathrm{~K}$, which is a significant improvement over conventional chemical reactors.

\section{Initial temperature and piston kinetic energy}

The change in results that occur when initial temperatures or maximum piston kinetic energy are changed are very similar to the results obtained with the partial oxidation experiments. The higher the maximum piston kinetic energy, the further the reactions progress. The higher the initial temperature, the less kinetic energy is needed to give the same result.

\subsubsection{Simulations of processes in the PCR}

The accuracy of the simulation results differ for different components and different processes, so the conclusions on various species and processes will be discussed separately.

\section{Main species by partial oxidation}

The major species formed in the partial oxidation process can be very well predicted by the simulations. There is some over prediction of the yield of hydrogen and carbon monoxide at high conversion levels, as well as an over prediction in the conversion of methane, which can be attributed to the absence of losses in the simulations. The moment of ignition, in terms of energy needing to be supplied, is slightly under predicted by the simulations.

The main difference between the different models used is the gap in terms of energy between the combustion like step and the steam reforming. The C3 and C5 models predict a smooth transition from the combustion like step to the steam reforming step, with no energy gap in between. The other three models, to a more or lesser extent, 
predict an energy gap between the combustion like step and the steam reforming step. Experimental results show that the prediction of the GRI3.0, Leeds and Konnov models are more accurate.

\section{Minor species by partial oxidation}

The difference between the predictions of different models is much larger when looking at the minor species yield. It is difficult to point to a real best model to predict the formation of minor species, but in general the Konnov model has the smallest deviation.

An attribute that is shared by all the minor species results is that the experimental data for high conversions is shifted along the methane conversion axis around $10-20 \%$. This difference between experiment and simulation is most likely caused by the fact that simulations are not affected by the presence of walls: all reactants are ideally mixed and the same temperature. In the experiments on the other hand, some of the reactants, those close to the wall, are cooled by the wall and do not react. The result is that the final composition of the product is a mixture of completely reacted gas from the core of the reactor and unreacted gas from the boundary layer. This effect manifests itself in a shift along the methane conversion axis: the unreacted methane dilutes the products.

\section{Hydrogen - carbon monoxide ratio by partial oxidation}

The hydrogen - carbon monoxide ratio is predicted well by the different models used. This is the case for both the shape of the hydrogen - carbon monoxide ratio as a function of methane conversion, as well as the absolute value of the ratio. The same shift in results that was found in the minor species comparison between experiments and simulations is found in the hydrogen - carbon monoxide ratio.

\section{Simulations as a design tool for partial oxidation processes}

Results from simulations show that they are well able to predict the process occurring in the PCR when partial oxidation of methane is the goal. The simulations also allow one to gain insight into what happens during one compression expansion stroke. It also shows that the PCR can generate super equilibrium concentrations of hydrogen from methane, giving yields of hydrogen that are higher than they would be in an adiabatic, isobaric flame that reacts to equilibrium.

\section{Main species by steam reforming}

The simulation mechanisms used are well capable to predict the yield of hydrogen in a PCR steam reforming cycle. However, the carbon monoxide concentration is grossly over predicted by all models. The over prediction of the carbon monoxide yield can be attributed to the absence of soot formation mechanism in the used models. This means 
that in processes where the soot formation is relatively high, a deviation is present in terms of the amount of carbon atoms present in gaseous species.

The difference in energy required for steam reforming to take place, which was previously observed in the partial oxidation simulations, becomes more important in the prediction of pure steam reforming. The C3 and C5 models predict steam reforming to occur with less energy input than other three models. Experiments show that the GRI3.0, Leeds and Konnov model are closer to the truth, but in fact also under predict the energy required for steam reforming.

\section{Minor species by steam reforming}

The ethane, ethylene and acetylene predictions support the conclusion that the steam reforming is preceded by a methane cracking step. In this step, hydrogen is formed trough the Kassel mechanism, while no carbon monoxide is formed yet.

In the prediction of minor species, the Konnov model can again be identified to be the most accurate predictor, although this time the performance of the Konnov model is more convincingly better. Yields of ethylene and acetylene are most well predicted by the Konnov mechanism, while the yield of ethylene on the other hand is predicted to be lower than the experimental results. In the case of ethylene the results correspond best to the Leeds mechanism. Experimental carbon dioxide is much lower than the predicted highest yield by all mechanisms.

\section{Hydrogen - carbon monoxide ratio}

Since the carbon monoxide yield is not predicted well by the models, the same can be expected for the hydrogen - carbon monoxide ratio. This becomes most evident in the comparison of experiments to simulation with initial temperature of $423 \mathrm{~K}$.

\section{Simulations as a design tool for steam reforming processes}

Based on the results obtained with the different mechanisms, compared to the experimental results, it can be concluded that the simulations cannot be used to accurately predict the steam reforming process in the PCR. The main reason is the absence of a soot model in the different mechanisms used. However, it must be said that it is unlikely that soot is a desirable product in the PCR, which means that it is unlikely that pure steam reforming, as it was performed in the experiments within this project, will ever be applied in practice. 


\subsection{The PCR as a chemical reactor for syngas generation}

The experimental and simulation results show that the different chemistry steps occur in a specific order (in terms of energy input required to initiate the step). The first step is the combustion like step, which is exothermic and requires the least energy to initiate. This is followed by the cracking of methane, which is (mostly) endothermic and requires more energy than is required to initiate the combustion like step. The last step, which requires the most energy to initiate, is the steam reforming step.

From the results obtained, it can be concluded that the process of syngas generation from methane by partial oxidation is a very suitable process for the PCR. Pure steam reforming on the other hand, when using the process parameters as done in this research, is much less applicable, due to the high soot yields in the preceding methane cracking step. Most likely, a mixed process, where both oxygen and steam are used as reactants to reform methane will give the best results. However, it is very likely that using different process parameters, like a higher initial temperature will greatly improve pure steam reforming results.

\subsection{The controllability and run-away possibility of the PCR}

The theory and experimental results show that for certain specific reactant flows into the PCR the process will converge to a stable operating point, as long as more energy is supplied than the unstable operating energy.

The approach presented gives clear insight in the controllability of the PCR operation: operation will either converge to a constant stable operating point, or cease. Though this might lead one to conclude that any process that has energy losses will be inherently stable and never run away, this depends heavily on the definition of runaway used. As energy losses are reduced, the stable operating point will move to a higher energy level. So, hypothetically, in a reactor with very little energy losses the process might be considered to run away (depending on the definition).

\subsection{Recommendations}

The results presented in this thesis and the conclusions based on them are a small step forward in making the PCR a industrially ripe, competitive alternative for conventional chemical reactors. However, much work needs to be done for Pulsed Compression technology to reach a stage of maturity that it can be wide applied. Some steps that could be taken to help reach this stage of maturity, in line with the work presented in this thesis are: 


\section{Investigate mixes of steam oxygen with a lighter piston}

The results obtained with pure steam reforming show that a significant amount of methane pyrolysis takes place when mixtures and process conditions as in the experiments are used. A way to improve the quality of the syngas manufactured and improve the carbon efficiency would be to reform with a mixture of oxygen and steam, with a lighter piston.

\section{Investigate more extreme cover geometries}

Investigations done in the automotive industry have shown that the effect of cover geometry (or piston geometry for that matter) are difficult to predict, and hard to optimize. It is very well possible that different, more extreme cover geometries will yield better results than the results obtained with the alternative cover geometries tested in this thesis.

\section{Apply continuous heat transfer measurement technique to a working reactor}

The continuous heat transfer measurement technique was shown to give interesting results on the PCR, but on in an operating regime that would be applicable for chemical reactions. It would be very interesting to apply the technique to a reactor that is actually operating under industrial conditions, with chemical reactions.

\section{Test different mechanisms, with soot model}

The comparison between experimental results and simulations showed that, for some operating conditions, the lack of a soot formation model in the mechanisms is a serious defect of the simulations done. A great improvement of the simulations would be to include a simple (but accurate) soot formation model.

\section{Include heat losses in the simulations}

While it was shown that heat losses do not influence results gravely, and good predictions can be made with the simulations as they are, an improvement of the models could be made by including heat losses from the model. This would improve the predictive power of the simulation model.

\section{Test different reactor geometries}

One of the parameters that was not tested in this project was the reactor geometry. One might consider, for example, a longer reactor or a reactor with a larger diameter. Information that is gained on the performance of different reactor geometries could ease the transition from experimental to industrial technology. 


\section{Simulate heavier pistons}

Perhaps against expectations, it was found that a heavier piston yielded identical results, when yields are compared based on piston kinetic energy. This may have been surprising, since a heavier piston has a significantly longer cycle time. It would therefore be interesting to simulate a compression expansion cycle with a heavier piston and see whether the yields are also the same in simulations. 



\section{Bibliography}

[1]. Levenspiel, O. The chemical reactor omnibook. s.I. : OSU book stores Corvallis, OR, 1996.

[2]. Evolution patterns and family relations in S-G reactors. van Swaaij, W.P.M., van der Ham, A.G.J., Kronberg, A.E. s.I. : Chemical Engineering Journal, 2002, Vols. 90 (2002) 2545.

[3]. Important roles of Fischer-Tropsch synfuels in the global energy future. Takeshita, T, Yamaji, K. s.I. : Energy Policy, 2008, Vols. 36 (2008) 2773-2784.

[4]. Energy and exergy analyses of a production process for methanol from natural gas. Rosen, M.A., Scott, D.S. s.I. : International Journal of Hydrogen Energy, 1988, Vols. 13 (1988) 617-623.

[5]. Petrochemicals from oil, natural gas, coal and biomass: Production costs in 20302050. Ren, T, Daniels, B, Patel, M.K., Blok, K. s.I.: Resources, Conservation and Recycling, 2009, Vols. 53 (2009) 653-663.

[6]. Kolbanovskii, Y A, Shchipachev, VS and Chernyak, N Y. Pulse compression of gases in chemistry and technology. s.I. : Nauka, Moscow, 1982.

[7]. Preparation of synthesis gas in the Otto engine with simultaneous power production. von Szeszich, L. 1956, Name: Chem.-Ing.-Tech, Vol. 28.

[8]. The production of hydrogen by the partial oxidation of methane in a dual fuel engine. Karim, GA and Moore, NPW. s.I. : SAE International, 1990.

[9]. Homogeneous charge compression ignition with a free piston: A new approach to ideal Otto cycle performance. Van Blarigan, P, Paradiso, $\mathbf{N}$ and Goldsborough, S. s.I. : Society of Automotive Engineers, 400 Commonwealth Dr, Warrendale, PA, 15096, USA, 1998.

[10]. Thermal and oxidative stability of fluorinated alkyl aryl ethers. Fukui, H, et al. s.I. : Elsevier, 2000, Journal of Fluorine Chemistry, Vol. 101, pp. 91--96. 
[11]. Effect of the chemical structure of fluorinated esters on the tribological properties of a steel-on-steel system. Zhu, JM and Liu, WM. s.I. : John Wiley \& Sons, 2004, Journal of Synthetic Lubrication, Vol. 21, pp. 223--230.

[12]. Combustion-Associated Wear in Alcohol-Fueled Spark Ignition Engines. Naegeli, D W. s.I. : SAE International, 1989.

[13]. Ichimiya, T and Takahashi, K. Wear Mechanisms of Methanol Fueled Engine. 1985. techreport.

[14]. Brutzkus, M. Apparatus for chemical production and research. s.l. : US Patent 1,586,508, 1926.

[15]. Glouchenkov, M J. s.I. : Russian patents: 2097121, 1997; 2115467, 1997; 2142844, 1999.

[16]. New trends in reforming technologies: from hydrogen industrial plants to multifuel microreformers. Ferreira-Aparicio, P, Benito, MJ and Sanz, Jl. s.l. : Taylor \& Francis, 2005, Catalysis Reviews, Vol. 47, pp. 491--588.

[17]. Pulsed compression reactor concept for synthesis gas production. Glouchenkov, M, et al. Berlin : XVI International Conference on Chemical Reactors, 2003.

[18]. Humphrey, H A. Apparatus for producing nitric oxide. s.I. : US Patent 1,429,035, 1922.

[19]. Broeze, J J and van Dijk,W J D. Reciprocating compression-reactor for short period, high temperature and high pressure chemical reactions. s.I. : US Patent 2,814,551, 1957.

[20]. van Dijk, J W D. Reciprocating compression-reactor for short period, high temperature and high pressure chemical reactions. s.I. : US Patent 2,814,552, 1957.

[21]. Kolbanovsky, Y A. s.I. : USSR Patens: 774.020, 1975; 880.460, 1978; 1.572.690, 1986; 1.696.741, 1988.

[22]. Herwig, O. Internal combustion engine for the production of synthesis gas. s.I. : US Patent: 2.864.297, 1954.

[23]. Glouchenkov, M J. Application of piston devices as chemical reactors. s.l.: Manuscript registered in the Russian society of intellectual rights, 1997. No 2145. 
[24]. Free piston pulsed compression reactor. Glouchenkov, M J, Kronberg, A E, Veringa, H. Venice: 4th International symposium on high pressure process technology and chemical engineering, 2002.

[25]. Combustion of light hydrocarbons in the free piston pulsed compression reactor. Glouchenkov, M J, Kronberg, A E and Veringa, A. Granada, Spain : 4th European congress of chemical engineering, 2003.

[26]. Pulsed compression: advanced technology for synthesis gas production. Glouchenkov, M J, Kronberg, A E. Brugge, Belgium : International conference gas fuel 05, 2005.

[27]. Pulsed compression technology: a breakthrough in production of hydrogen. Glouchenkov, M J, Kronberg, A E. Lyon, France: 16th World Hydrogen Energy Conference, 2006.

[28]. Pulsed compression: advanced technology for high temperature, high pressure processes. Glouchenkov, M J, Kronberg, A E. Potsdam, Germany: 19th International symposium on chemical reaction engineering, 2006.

[29]. The free-piston engine development - Present status and design aspects. London A.L., Oppenheim A.K.,. s.I. : Transactions of the ASME, 1952, Vols. 1952:74:1349-1361.

[30]. Predictive piston motion control in a free-piston internal combustion engine. Mikalsen, R, Jones, E and Roskilly, AP. s.I. : Elsevier, 2009, Applied Energy.

[31]. Prototype testing and analysis of a novel internal combustion linear generator integrated power system. Xu, Z and Chang, S. s.I. : Elsevier, 2010, Applied Energy, Vol. 87, pp. 1342--1348.

[32]. A review of free-piston engine history and applications. Mikalsen, R. and Roskilly, A.P. Newcastle upon Tyne: Applied Thermal Engineering, 2007, Vols. 27 (14-15), pp. 2339-2352 .

[33]. Miniature free-piston homogeneous charge compression ignition engine-compressor concept--Part I: performance estimation and design considerations unique to small dimensions. Aichlmayr, HT, Kittelson, DB and Zachariah, MR. s.I.: Elsevier, 2002, Chemical Engineering Science, Vol. 57, pp. 4161--4171. 
[34]. A review of free piston engine concepts. Achten, P.A.J. s.I. : SAE paper 941776, 1994.

[35]. Free-Piston Diesel Engine Timing and Controlâ€"Toward Electronic Cam-and Crankshaft. Johansen, T A, et al. 2002, IEEE Transactions on Control Systems Technology, Vol. 10, p. 177.

[36]. Investigation of linear generator starting modes by mechanical resonance and rectangular current commutation. Zulkifli, S A and A-Aziz, A R. 2009.

[37]. The control of a free-piston engine generator. Part 1: Fundamental analyses. Mikalsen, R and Roskilly, AP. s.I. : Elsevier, 2010, Applied Energy, Vol. 87, pp. 1273-1280.

[38]. The control of a free-piston engine generator. Part 2: Engine dynamics and piston motion control. Mikalsen, R and Roskilly, AP. s.I. : Elsevier, 2010, Applied Energy, Vol. 87, pp. 1281--1287.

[39]. Coupled dynamic-multidimensional modelling of free-piston engine combustion. Mikalsen, R and Roskilly, AP. s.I. : Elsevier, 2009, Applied Energy, Vol. 86, pp. 89--95.

[40]. Micro-HCCl combustion: experimental characterization and development of a detailed chemical kinetic model with coupled piston motion. Aichlmayr, HT, Kittelson, DB and Zachariah, MR. s.I. : Elsevier, 2003, Combustion and Flame, Vol. 135, pp. 227--248.

[41]. The production of hydrogen through the uncatalyzed partial oxidation of methane in an internal combustion engine. Karim, G.A., Wierzba, I. s.I. : International journal of hydrogen energy, 2008, Vols. 33 (2008) 2105-2110.

[42]. A rapid compression machine for chemical kinetics studies at elevated pressures and temperatures. Mittal, G and Sung, C J. s.I. : Taylor \& Francis, 2007, Combustion Science and Technology, Vol. 179, pp. 497--530.

[43]. Autoignition of $\mathrm{H} 2 / \mathrm{CO}$ at elevated pressures in a rapid compression machine. Mittal, G, Sung, C J and Yetter, R A. s.I. : John Wiley \& Sons, 2006, International Journal of Chemical Kinetics, Vol. 38, pp. 516--529.

[44]. Ignition properties of methane/hydrogen mixtures in a rapid compression machine. Gersen, S, et al. s.I. : Elsevier, 2008, International Journal of Hydrogen Energy, Vol. 33, pp. 1957--1964. 
[45]. Direct conversion of methane to acetylene or syngas at room temperature using non-equilibrium pulsed discharge* 1. Kado, S, et al. s.l. : Elsevier, 2003, Fuel, Vol. 82, pp. 1377--1385.

[46]. Manufacture of Synthesis Gas by Homogeneous Oxidation of Methane. Sister, VG, Bogdanov, VA and Kolbanovskii, Y A. s.I. : Oxford; New York: Pergamon Press, 1992-, 2005, Petroleum Chemistry, Vol. 45, pp. 407--412.

[47]. Experimental and modeling study of hydrogen/syngas production and particulate emissions from a natural gas-fueled partial oxidation engine. McMillian, $\mathbf{M} \mathbf{H}$ and Lawson, S A. s.l. : Elsevier, 2006, International Journal of Hydrogen Energy, Vol. 31, pp. 847--860.

[48]. The syngas production by partial oxidation using a homogeneous charge compression ignition engine. Yang, Y C, Lim, M S and Chun, Y N. s.l. : Elsevier, 2009, Fuel Processing Technology, Vol. 90, pp. 553--557.

[49]. Uddeholm Corrax. [Online] Uddeholm. http://www.bucorp.com/files/UDDEHOLM_CORRAX.pdf.

[50]. Poco Graphite, Inc. ACF-10Q. [Online] Poco Graphite, Inc. [Cited: September 9, 2010.] http://www.poco.com/MaterialsandServices/Graphite/IndustrialGrades/ACF10Q/tabid/ 88/Default.aspx.

[51]. Tanso, Toyo. Characteristics of Mechanical carbon. [Online] Toyo Tanso. [Cited: September 9, 2010.] http://www.toyotanso.co.jp/Products/Mechanical_carbon_detail_en.html.

[52]. Oude Nijeweme, D.J. Unsteady in-cylinder heat transfer in a spark ignition engine: experiments and modelling. Enschede : University of Twente, master's thesis, 2000.

[53]. Wang, Xiaowei. Instantaneous In-cylinder Heat Transfer and Combustion Analysis in Spark Ignition Engines. Oxford : s.n., 2008.

[54]. An experimental Investigation of Surface Thermometry and Heat Flux. Gatowski, J.A., Smith, M.K. and Alkidas, A.C. Michigan : Experimental Thermal and Fluid Science, 1989, Vols. 2:280-292. 
[55]. Hoogendoorn, C.J. and Meer, T.H. van der. Fysische transportverschijnselen II (Physical transport phenomena II). 1991 (reprint 2007).

[56]. Thermal diffusivity and thermal conductivity of Chromal, Alumel and Constantane in the range 100-450K. Sundqvist, B. Umea : Journal of Applied Physics, 1992, Vol. 72 (2).

[57]. Hirschfelder, J.O., Curtiss, C.F., and Bird, R.B. Molecular theory of gasses and liquids. New York : Wiley, 1964.

[58]. Uber das Warmeleitvermogen, die spezifische Warme und die innere Reibung von Gasen. Eucken, A. s.I. : Phys. Z., 1913, Vols. 14 pp. 324-332.

[59]. E.W. Lemmon, M.O. McLinden and D.G. Friend. NIST Chemistry WebBook, NIST Standard Reference Database Number 69. [Online] [Cited: March 26, 2010.] http://webbook.nist.gov.

[60]. High Reynolds number flow in a moving corner. Tabaczynski, R.J., Hoult, D.P. and Keck, J.C. s.I. : J. Fluid Mech., 1970, Vols. 42, part 2, pp. 249-255.

[61]. Temperature fields during the development of combustion in a rapid compression machine. Griffiths, J.F., Clarkson, J., Macnamara, J.P. and Whitaker, B.J. s.I.: Combustion and Flame, 2001, Vols. 125 (3), pp. 1162-1175.

[62]. Rapid compression machines: Heat transfer and suppression of corner vortex. Lee, D. and Hochgreb, S. s.l. : Combustion and Flame, 1998, Vols. 114 pp. 531-545.

[63]. The thermal decomposition of methane. Kassel, Louis S. Pittsburg : Journal of the American Chemical Society, 1932, Vols. 54 (10), pp. 3949-3961.

[64]. Verbeek, A.A. Synthesis gas production by Pulsed Compression Technology. Enschede : University of Twente, master's thesis, 2010.

[65]. Smith, G.P. [Online] http://www.me.berkley.edu/gri_mech/.

[66]. Methane/propane oxidation at high pressures: experimental and detailed chemical kinetic modeling. Petersen, E.L., Kalitan, D.M., Simmons, S., Bourque, G, Curran, H.J. and Simmie, J.M. s.I. : Proceedings of the combustion institute, 2007, Vols. 31 I, pp. 447454.

[67]. Ignition and flame speed kinetics of two natural gas blends with high levels of heavier hydrocarbons. Bourque, G., Healy, D., Curran, H., Zinner, C., Kalitan, D., Vries, 
de, J., Aul, C., Petersen, E. s.I. : Proceedings of the ASME Turbo Expo , 2008, Vols. (PART B), pp. 1051-1066.

[68]. Development and testing of a comprehensive chemical mechanism for the oxidation of methane. Hughes, K.J., Turanyi, T., Clague, A.R., Pilling, M.J. Leeds : International Journal of Chemical Kinetics, 2001, Vols. 33 (9), pp. 513-538.

[69]. Development and validation of a detailed reaction mechanism for the combustion of small hydrocarbons. Konnov, A.A. Edinburgh : 28-th Symposium (Int.) on Combustion, 2000, Vol. Abstr. Symp. Pap. p. 317.

[70]. Pressure dependence of the auto-ignition temperature of methane/air mixtures.

Caron, M, et al. s.I. : Elsevier, 1999, Journal of hazardous materials, Vol. 65, pp. 233--244.

[71]. Aichlmayr, H.T. Design considerations, modeling, and analysis of microhomogeneous charge compression ignition combustion free-piston engines. University of Minnesota : s.n., 2002.

[72]. Horsepower with brains: the design of the chiron free piston engine. Achten, P.A.J., Oever, J.P.J. van den and Potma, J. s.I. : SAE paper 200-01-2545, 2000.

[73]. A thin-film thermocouple for transient heat transfer measurements in ceramiccoated combustion chambers. Assanis, D.N. and Friedman, F.A. Urbana: Pergamon Press Ltd, 1993, Vols. 20, pp. 459-468.

[74]. Thermo reflectance bolometry. Mitko, S.V., Oudalov, A.Yu., Udalov, Yu.B., Peters, P.J.M. and Boller, K.J. s.I. : American Institute of Physics, 2004, Vol. 76.

[75]. World Bank, The. Global gas flaring reduction partnership: towards a world free of flares. [Online] http://www.worldbank.org/ggfr.

[76]. Manufacture of hydrogen. Rostrup-Nielsen, T. s.I. : Elsevier, 2005, Catalysis Today, Vol. 106, pp. 293--296.

[77]. On the controllability and run-away possibility of a totally free piston, pulsed compression reactor. Roestenberg, T, et al. s.l. : Elsevier, 2010, Chemical Engineering Science.

[78]. Heat transfer study of the pulsed compression reactor. Roestenberg, T, et al. s.l. : Elsevier, 2010, Chemical Engineering Science, Vol. 65, pp. 88--91. 
[79]. Kinetics modeling of shock-induced ignition in low-dilution $\mathrm{CH} 4 / \mathrm{O} 2$ mixtures at high pressures and intermediate temperatures. Petersen, EL, Davidson, DF and Hanson, RK. s.l. : Elsevier, 1999, Combustion and flame, Vol. 117, pp. 272--290.

[80]. Rapid compression machine measurements of ignition delays for primary reference fuels. Park, P and Keck, J C. s.l. : SAE International, 1990.

[81]. A rapid compression machine investigation of oxidation and auto-ignition of $n$ heptane: Measurements and modeling. Minetti, R, et al. s.l. : Elsevier, 1995, Combustion and Flame, Vol. 102, pp. 298--309.

[82]. Cost to Make CO or H2+ CO. Michel, S. 1989, Hydrocarbon Processing, Vol. 68, p. 37.

[83]. Mantzos, L, et al. European energy and transport trends to 2030. s.l. : European Communities, 2003.

[84]. Krediet, H.J. A heat transfer study on a Pulsed Compression Reactor. Enschede : University of Twente, master's thesis, 2008.

[85]. Houghton, J T. Climate change 1995: the science of climate change. s.l. : Cambridge Univ Pr, 1996.

[86]. Pulsed compression reactor. Glouchenkov, M J, Kronberg, A E. University of Bath, U.K.: Proceedings of the 3rd International Symposium on Multifunctional Reactors, 2003.

[87]. Catalysis in C 1 chemistry: Future and prospect. Fierro, JLG. s.I. : Springer, 1993, Catalysis Letters, Vol. 22, pp. 67--91.

[88]. New trends in reforming technologies: from hydrogen industrial plants to multifuel microreformers. Ferreira-Aparicio, P, Benito, MJ and Sanz, JL. s.l. : Taylor \& Francis, 2005, Catalysis Reviews, Vol. 47, pp. 491--588.

[89]. On the Kinetics and Reactor Modelling of the Steam Reforming of Methane - A Review. Elnashaie, S, et al. 1988, J. Eng. Sci, Vol. 14, p. 247.

[90]. A review of catalytic partial oxidation of methane to synthesis gas with emphasis on reaction mechanisms over transition metal catalysts. Christian Enger, B, LÃ,deng, R and Holmen, A. s.I. : Elsevier, 2008, Applied Catalysis A: General, Vol. 346, pp. 1--27. 
[91]. Carslaw, H.S., Jaeger, J.C. Conduction of heat in solids, 2nd edition. Oxford: Clarendon press, 1959.

[92]. Simulation of methane autoignition in a rapid compression machine with creviced pistons. Brett, L, et al. s.I. : New York: Elsevier, 1957-, 2001, Combustion and Flame, Vol. 124, pp. 326--329.

[93]. Boden, T and Marland, G. Global CO2 Emissions from Fossil-Fuel Burning, Cement Manufacture, and Gas Flaring: 1751-2007. [Online] Carbon Dioxide Information Analysis Center. http://cdiac.ornl.gov/ftp/ndp030/global.1751_2007.ems.

[94]. Technologies for large-scale gas conversion. Aasberg-Petersen, $\mathbf{K}$, et al. s.l.: Elsevier, 2001, Applied Catalysis A: General, Vol. 221, pp. 379--387. 



\section{Acknowledgements}

There are a few people that I wish to thank for various reasons:

Liliane, for being the most wonderful person that you are, moving to Enschede to give me the opportunity to do this $\mathrm{PhD}$, agreeing to marry me and just being you. You never cease to amaze and surprise me.

My parents, for putting me on this world, stimulating me to be the best I can be, proofreading the occasional publication and generally being the best parents I could wish for.

Theo, for offering me the opportunity to do this $\mathrm{PhD}$, for all your advice, your guidance, your sense of humor and for making your garden available for the yearly barbeque.

Maxim and Sasha, for being brilliant inventors and for your inexhaustible fountain of ideas and suggestions.

Sander and Frans, for your guidance and advice and for taking the time to travel to Enschede every month to discuss progress and problems.

All the members of my PhD committee, for taking the time to read my work and coming to Enschede for my defense.

Uros, Marc, Anton and Santosh, for being great office mates, with a great sense of humor and practical jokes. For making my stay at the UT generally great.

Henk-Jan and Willie, for all your technical support and help in the lab.

All the students that did their work under my guidance, for providing new insights, measurement data, food for though and many great ideas.

All the people and students of the thermal engineering group that I haven't mentioned, for being a great bunch of colleagues and for putting up with all the practical jokes our office pulled on you.

The guys from de Vlam and Rotor soccer team, for winning the UT cup, twice, and providing a great energetical outlet.

My friends and pokerbuddies, for all the great pokernights, barbeques and nights out into town.

Liliane, because you deserve to be thanked twice. 



\section{Different high speed surface temperature sensors}

\section{Thin film thermocouples}

The working, merits and drawbacks of thin film thermocouples, can be explained well by the design proposed by Assanis and Friedmann [73]. The temperature sensor assembly consists of a cast-iron plug body and a deposited thin film sensing junction. To manufacture this sensor chromel and alumel thermocouple wires are run through the plug body, approximately $5 \mathrm{~mm}$ apart. Wire insulating ceramic sleeves are used to insulate the wires from the body. The wires are cemented in place such that they protrude $1.0 \mathrm{~mm}$ beyond the surface of the plug. A schematic of the plug at this stage in the fabrication is shown in Figure I.1 (A).

Subsequently, a plasma-sprayed, partially- stabilized zirconia (PSZ) coating is applied to the surface of the plug. The ceramic coating thickness is $1.5 \mathrm{~mm}$, covering the protruding thermocouple wires. A schematic of the plug at this stage in the fabrication is shown in Figure I.1 (B). Excess coating is ground away to expose the thermocouple lead wires. The surface is then polished and cleaned in preparation for the last step in the process. The plug at this stage in the fabrication is shown in Figure I.1 (C). The last step of the process is the deposition of the two metal films by ion plating process. These metal films form the thermocouple junction between and are between 0.8 and $1.0 \mu \mathrm{m}$. A schematic of the final assembly is shown in Figure I.1 (D).

\begin{tabular}{ccc}
\hline \hline Parameter value & Upper limit calculation & Lower limit calculation \\
\hline$\alpha$ & $4.68 \cdot 10^{-6} \mathrm{~m}^{2} \cdot \mathrm{s}$ & $7.34 \cdot 10^{-6} \mathrm{~m}^{2} \cdot \mathrm{s}$ \\
$\mathrm{L}$ & $1.0 \cdot 10^{-6} \mathrm{~m}$ & $0.8 \cdot 10^{-6} \mathrm{~m}$ \\
Fo & 10 & 1 \\
\hline $\mathrm{t}$ & $2.13 \cdot 10^{-6} \mathrm{~s}$ & $8.72 \cdot 10^{-8} \mathrm{~s}$ \\
\hline \hline
\end{tabular}

Table I.1, Calculation of the upper- and lower limit of the response time of a thin film thermocouple 

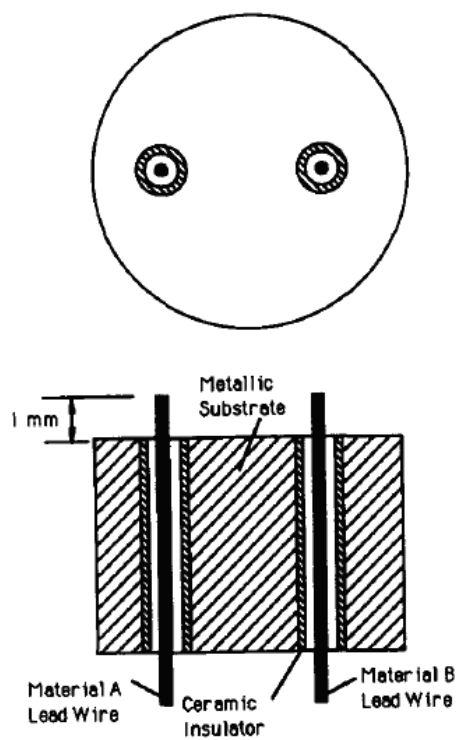

(A)

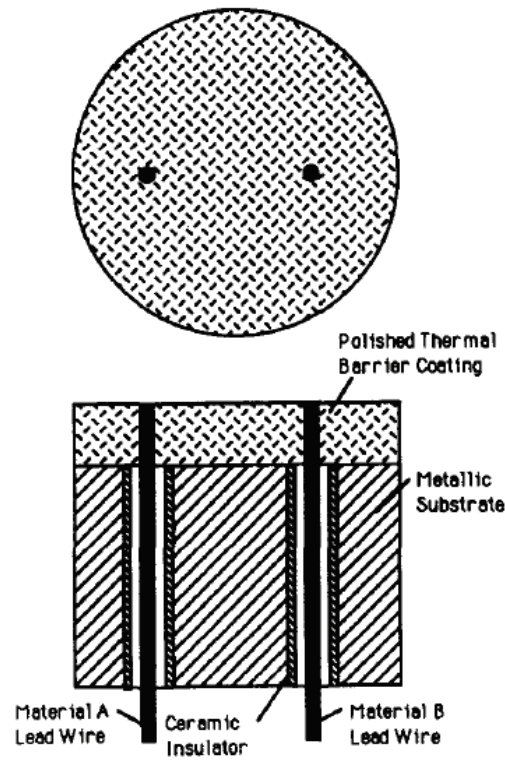

(c)

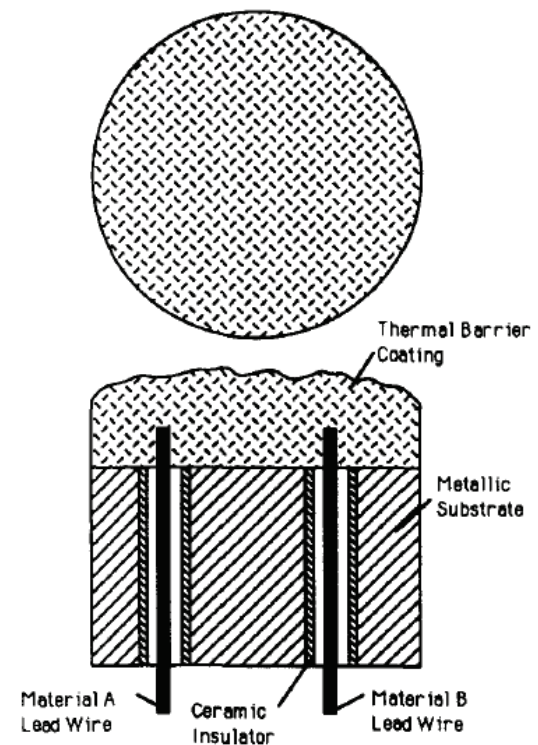

(B)

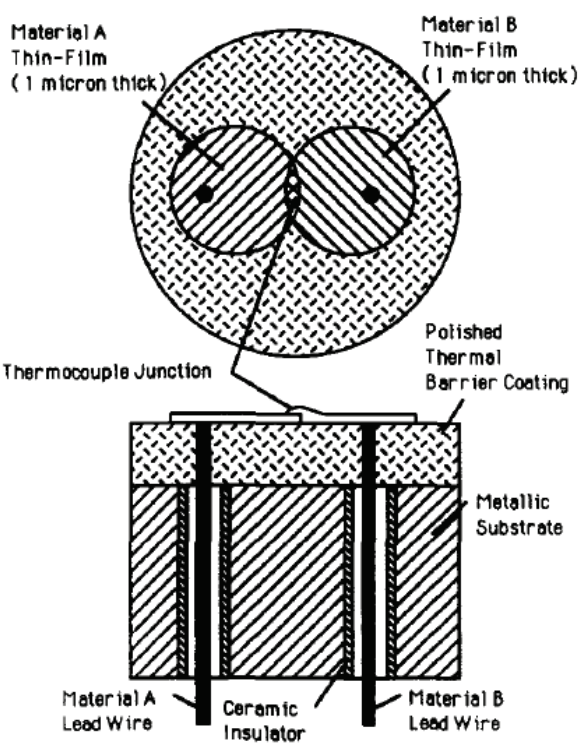

(D)

Figure I.1, Schematic view of the fabrication process of a thin film thermocouple [73] 
The upper and lower limit of the response time of such an assembly can be calculated using the thermal diffusivity of either chromel or alumel (depending on which material is deposited last), the upper and lower limit of the thickness of the metal films and calculating the time it takes to reach a Fourier number of 1 or 10 . When the thermal diffusivity of chromel (which is the lower of the two, taken from [56]) and the metal film thickness of $1.0 \mu \mathrm{m}$ is used to calculate the time to reach Fourier number 10, the upper limit of the response time is found. When the thermal diffusivity of alumel (which is the higher of the two, taken from [56]) and the metal film thickness of $0.8 \mu \mathrm{m}$ is used to calculate the time to reach Fourier number 1 , the lower limit of the response time is found. This is briefly summarized in Table I.1. In this table it can be seen that the upper limit of the response time is $2.13 \cdot 10^{-6} \mathrm{~s}$ and the lower limit of the response time for this type of sensor is $8.72 \cdot 10^{-8} \mathrm{~s}$. This means that the response time of the thin film thermocouple is quite close to the required response time for the measurements in the PCR and thin film thermocouples might, in a worst case scenario, not entirely be able to follow the rapid surface temperature changes as they occur.

Other important aspects of thin film thermocouples that influence the choice of whether or not they are applicable to PCR measurements, besides the response time, are their cost and durability. Manufacturing thin film thermocouples is quite labor intensive, where quite a lot of expensive equipment is needed. Since they are not so widely used, they are not mass produced, and thus relatively expensive. The sensor itself is also not extremely durable. The actual measurement surface is exposed, and very fragile. Once the sensor is broken, it cannot be repaired and must be replaced.

\section{Thin film thermistors}

The design of a thin film thermistor is quite similar to that of a thin film thermocouple. The difference is that in the case of a thermistor only one material is used for the leads and only one material is applied to the surface, to connect the leads (though these materials do not necessarily have to be the same). Instead of measuring the potential difference over the leads, as would be done with a thermocouple, the electrical resistance is measured. This resistance of the system is, if designed well, mainly dependant on the resistance of the thin film on the surface. When the temperature of this film changes, this influences the resistance of the film, which in turn influences the resistance of the system.

Since the total resistance of the system is a summation of the different parts working in series (the lead, then the thin film, then another lead), the working of the thin film can be optimized by making the thin film electrical resistance relatively high, while giving the 
leads a relatively low electrical resistance. This can be done by, for example, using a meander structure for the thin film, so that the effective length of the thin film connecting the leads is increased. This is schematically shown in Figure I.2.

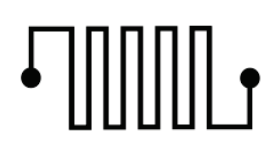

Figure I.2, Example meander structure that could be used to optimize a thin film thermistor

While using a meander structure for the thin film may have very beneficial effects on the working of the thin film thermistor, it makes the device even more expensive to manufacture. The response time of a thin film thermistor is generally comparable to that of a thin film thermocouple.

\section{Thermo reflectance bolometry}

Thermo reflectance bolometry is a technique where the measurement of the optical reflectivity change of a thin metal layer deposited on a transparent substrate is used to determine the temperature change of this metal layer. This concept was described by Mitko et al. in [74]. In Figure I.3 a schematic view of the setup used by Mitko et al. is shown. 


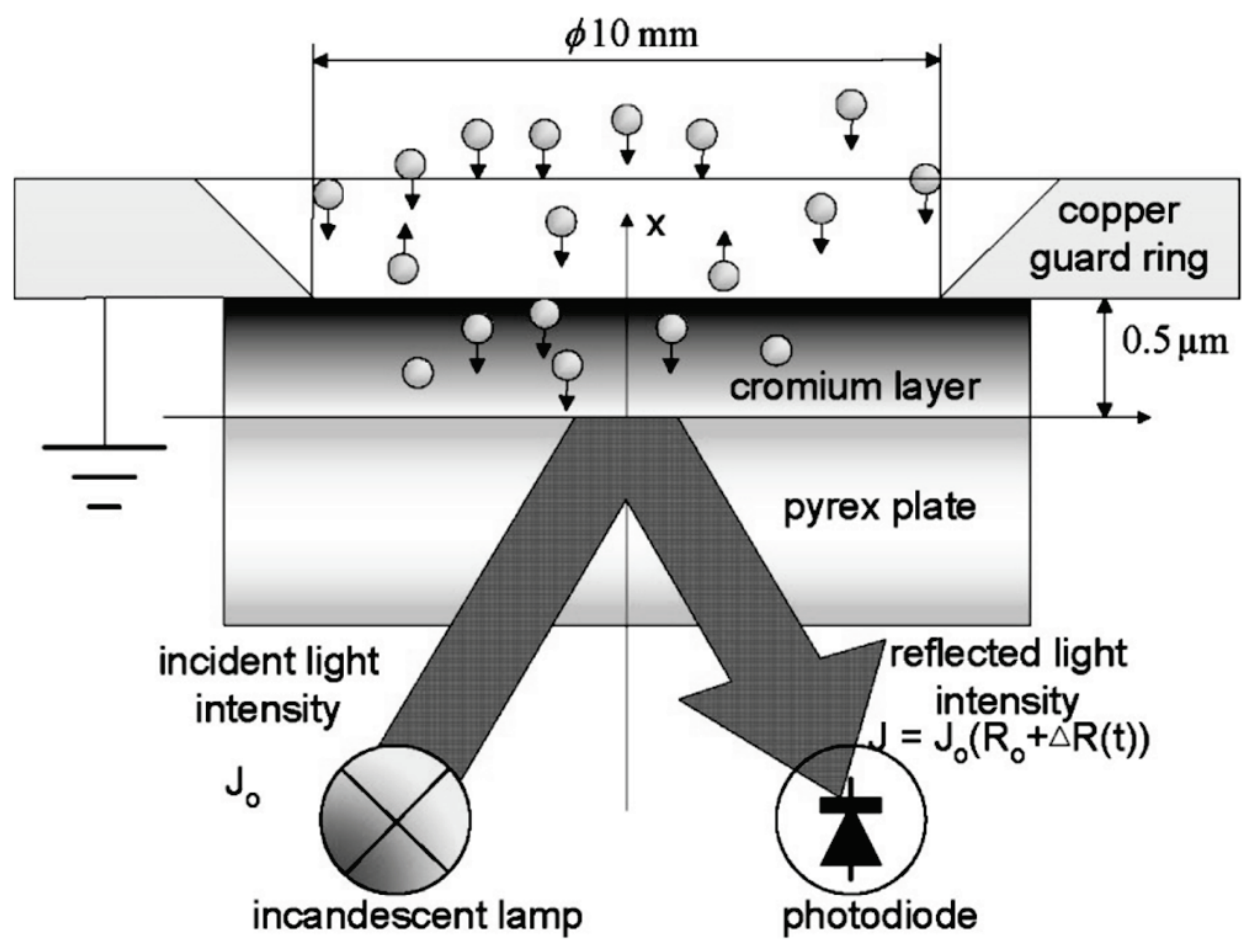

Figure I.3, Schematic view of thermo reflectance bolometry [74]

The sensor of the bolometer has no ohmic contact with the measuring unit, making this method well suited for an environment with strong electromagnetic noise interference. The response time is, again, dependent mainly on the thickness of the metallic layer. This also means that the response time is comparable to that of thin film thermocouples and thin film thermocouples. 



\section{Yields with alternative cover designs}

The following graphs show the results obtained with the alternative cover designs.

Results are ordered according to cover design and temperature, first showing all hydrogen yield graphs, then all carbon monoxide yield graphs. 

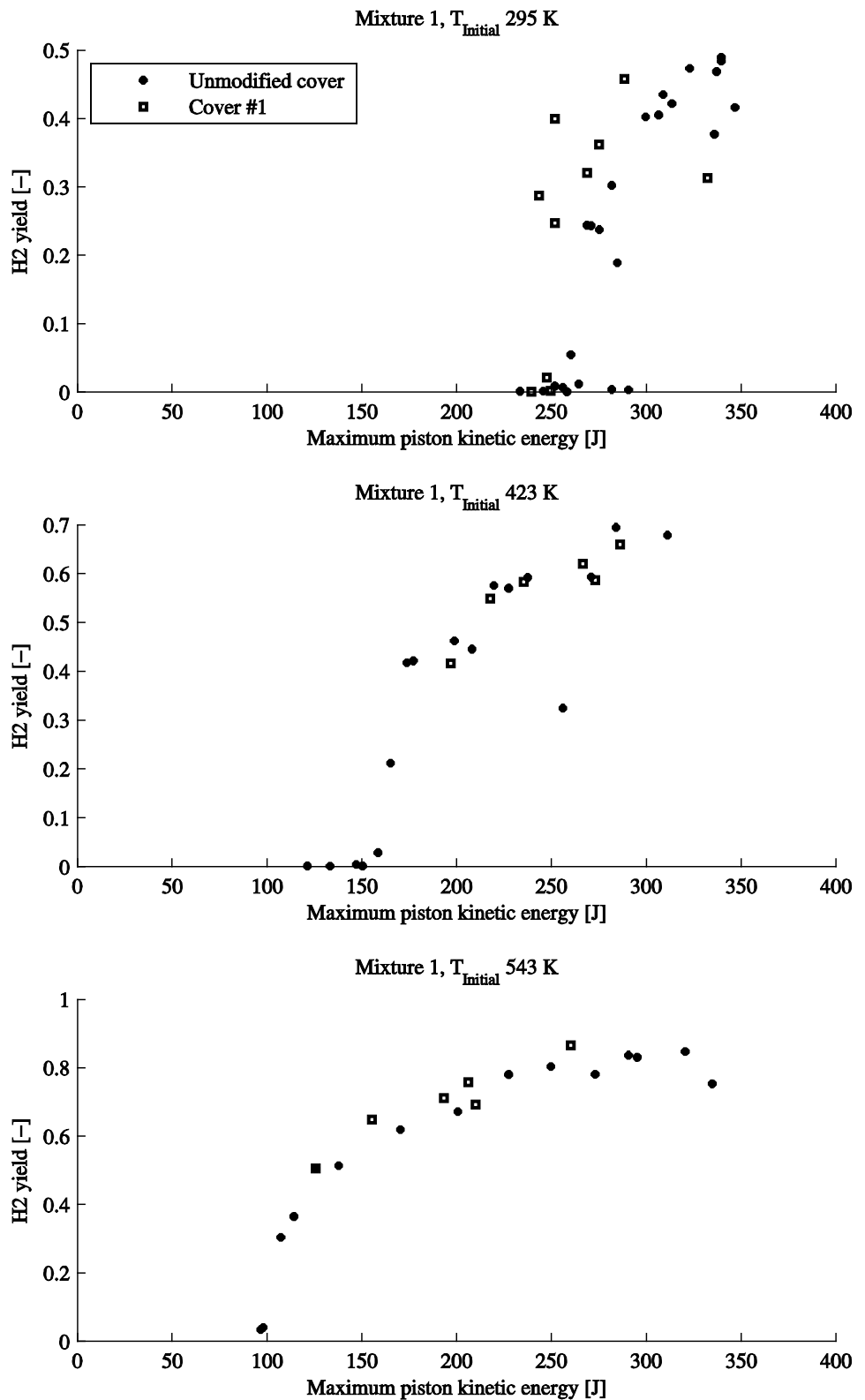

Figure II.1, Hydrogen yield of experiments with cover 1, with mixture 1 and different initial temperatures 

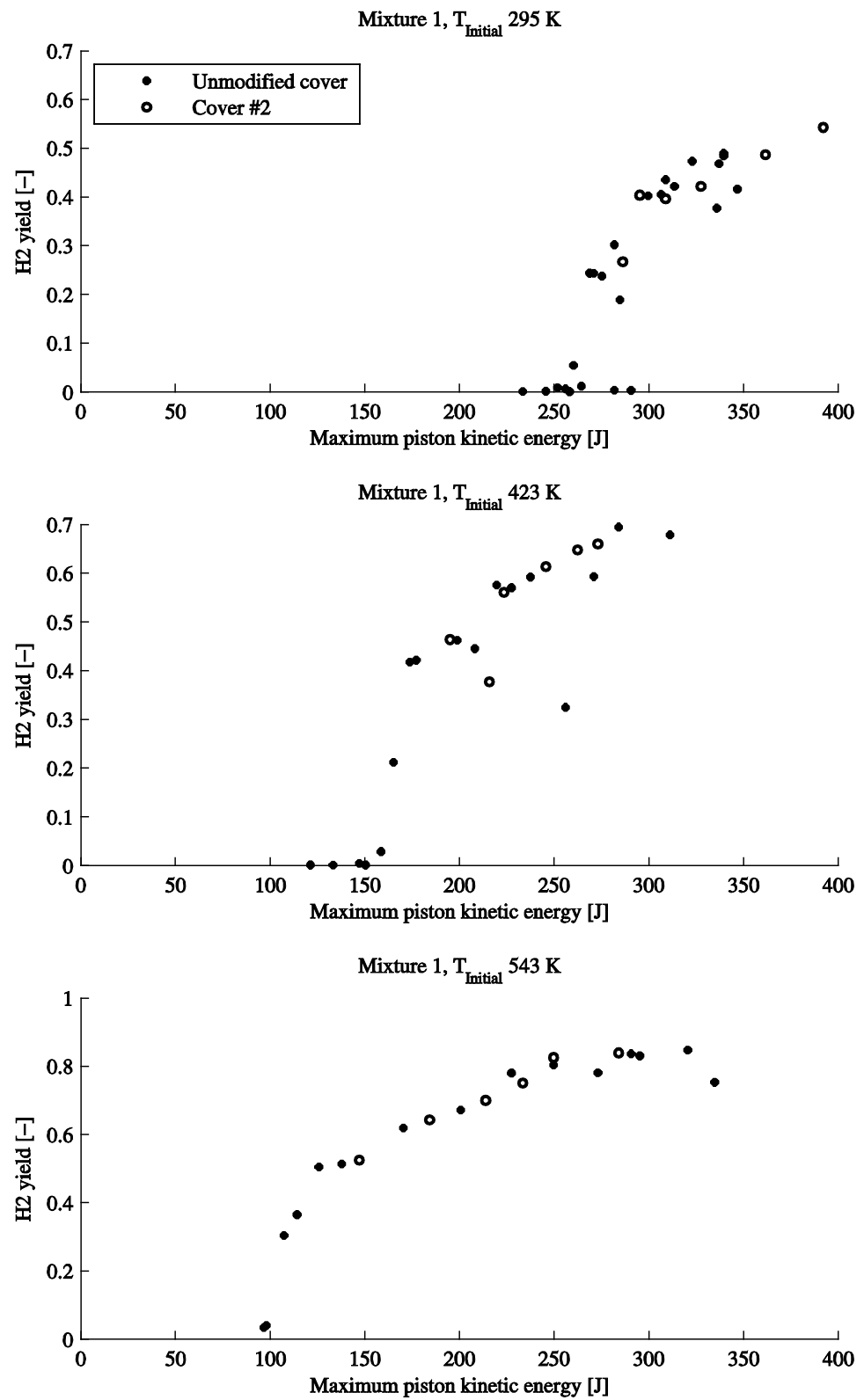

Figure II.2, Hydrogen yield of experiments with cover 2, with mixture 1 and different initial temperatures 

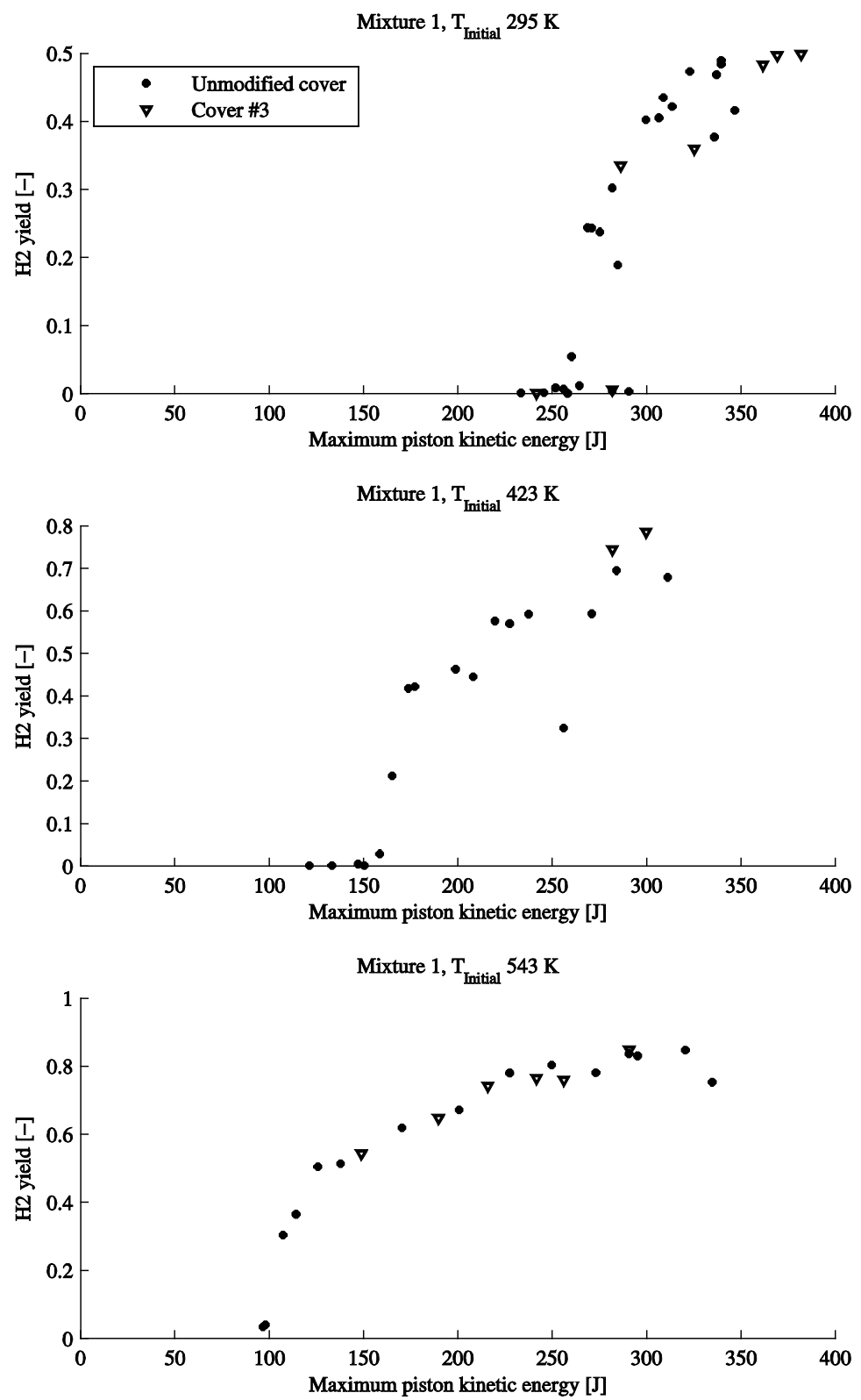

Figure II.3, Hydrogen yield of experiments with cover 3, with mixture 1 and different initial temperatures 

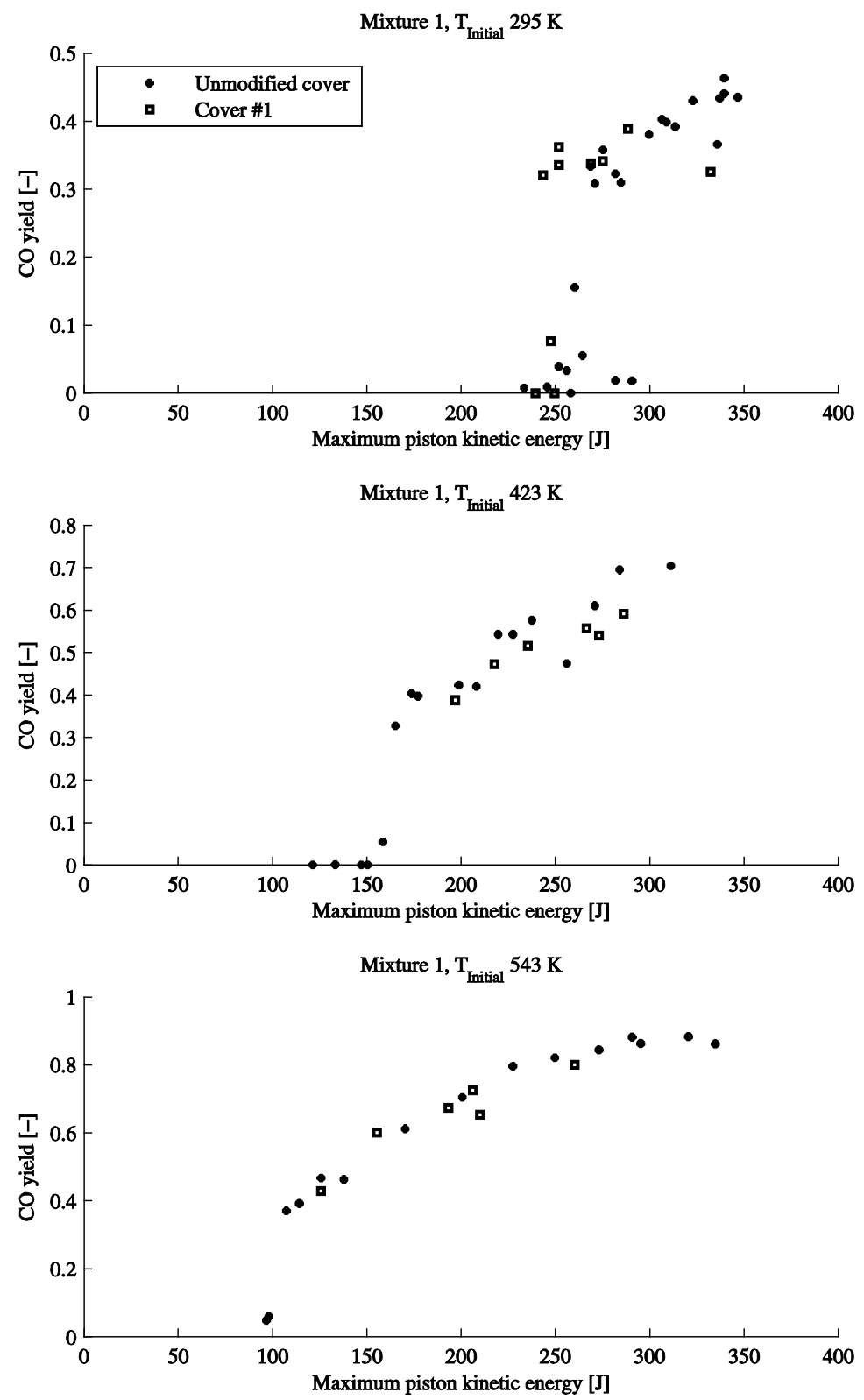

Figure II.4, Carbon monoxide yield of experiments with cover 1, with mixture 1 and different initial temperatures 

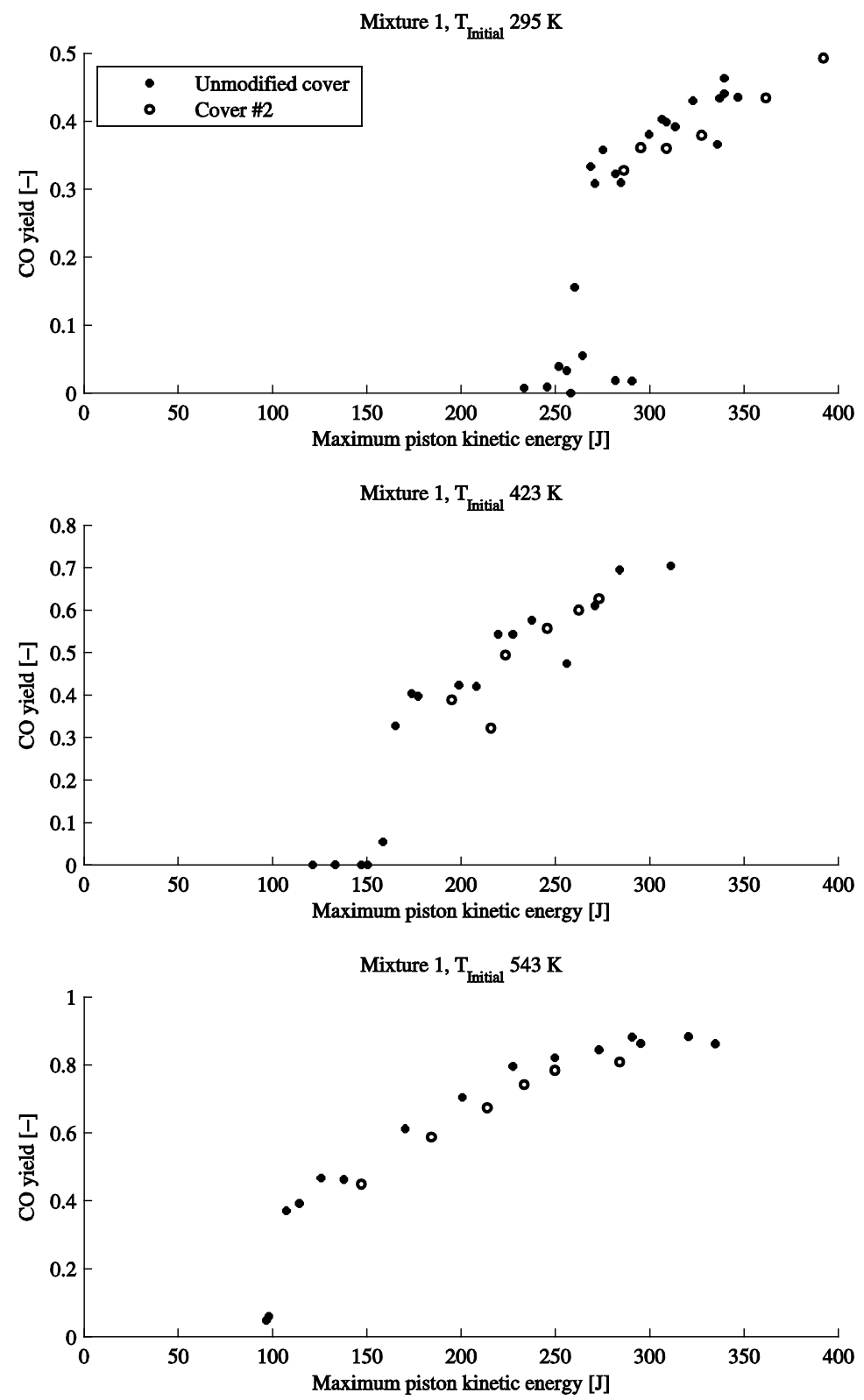

Figure II.5, Carbon monoxide yield of experiments with cover 2, with mixture 1 and different initial temperatures 

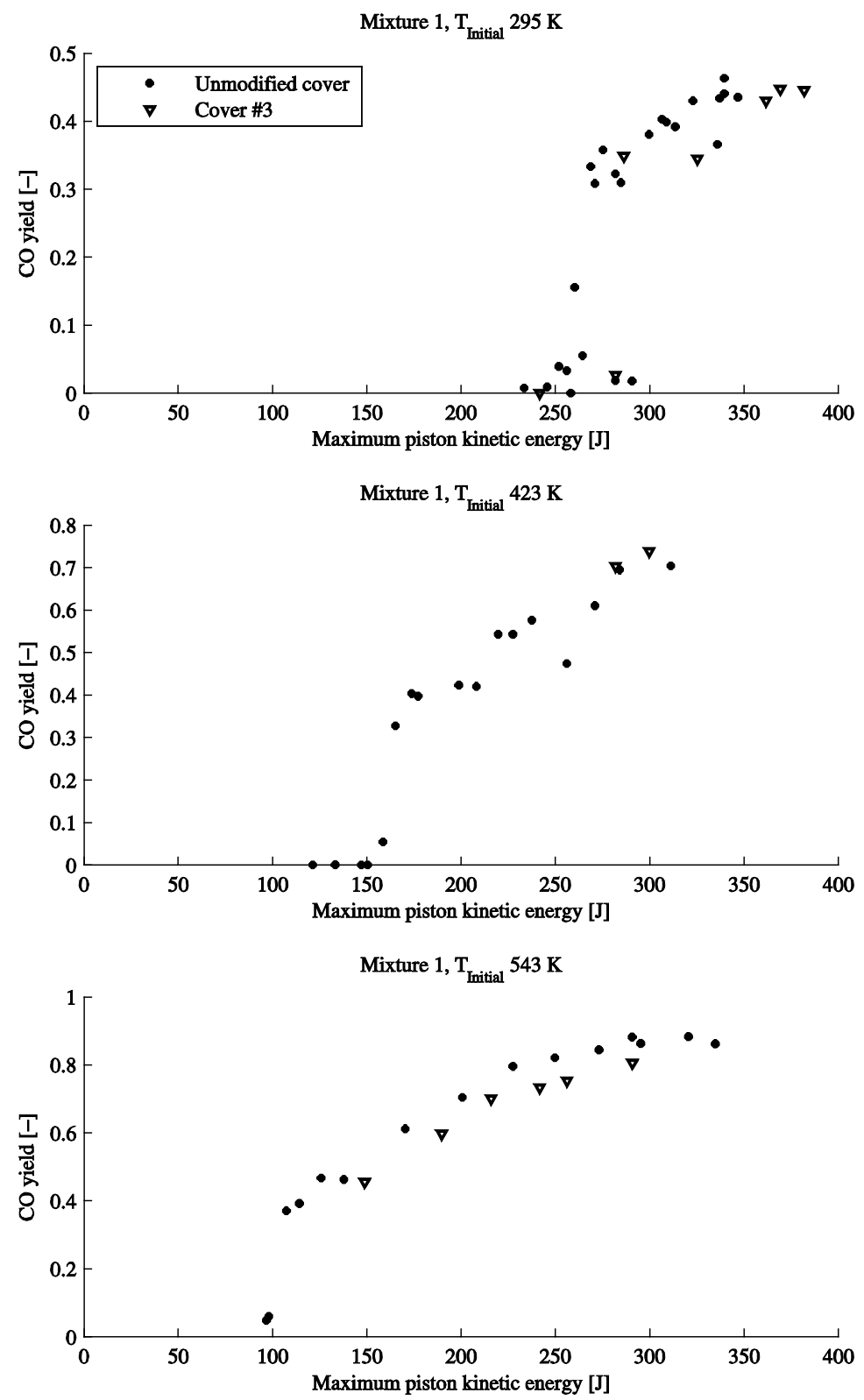

Figure II.6, Carbon monoxide yield of experiments with cover 3, with mixture 1 and different initial temperatures 